\title{
Oxidative Rearrangement of MIDA ( $N$-Methyliminodiacetic Acid) Boronates: Mechanistic Insights and Synthetic Applications
}

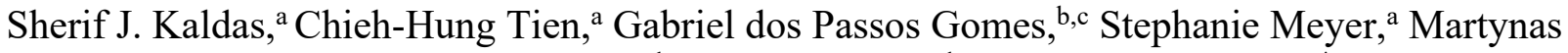
Sirvinskas, ${ }^{\mathrm{a}}$ Hayden Foy, ${ }^{\mathrm{d}}$ Travis Dudding, ${ }^{\mathrm{d}}$ and Andrei K. Yudin ${ }^{\mathrm{a}^{*}}$

aDavenport Research Laboratories, Department of Chemistry, University of Toronto, 80 St. George St. Toronto, ON, Canada, M5S 3H6

${ }^{\mathrm{b} C h e m i c a l}$ Physics Theory Group, Department of Chemistry, University of Toronto, Toronto, Ontario, M5S 3H6, Canada

${ }^{\mathrm{c}}$ Department of Computer Science, University of Toronto, 214 College St., Toronto, Ontario M5T 3A1, Canada.

${ }^{\mathrm{d}}$ Department of Chemistry, Brock University, 1812 Sir Isaac Brock Way, St. Catharines, ON L2S 3A1, Canada 


\section{Table of Contents}

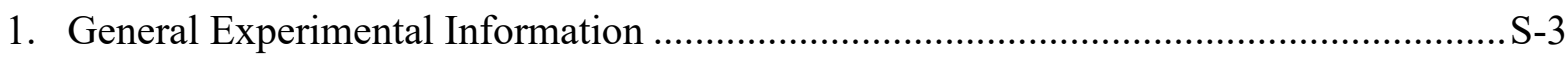

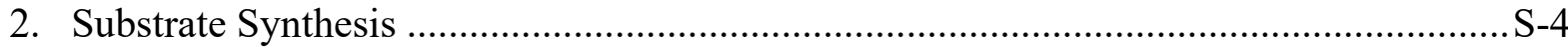

3. Oxidative Rearrangement Optimization Studies.........................................................

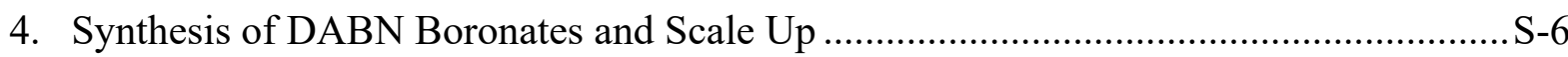

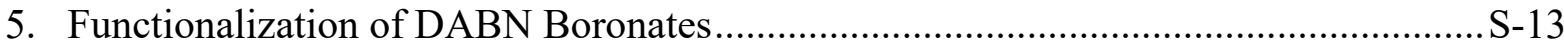

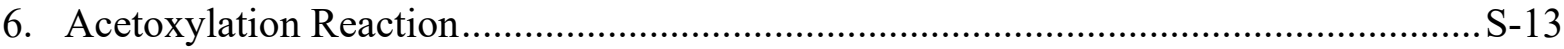

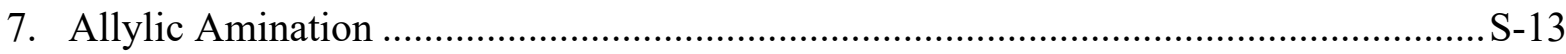

8. Arylation and Azidation........................................................................................ S-14

9. 3-Component Coupling ............................................................................................ S-15

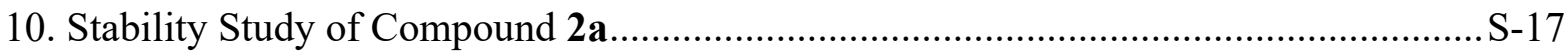

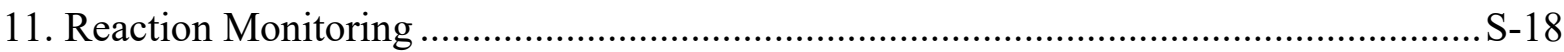

12. General Information for DFT Calculations ………............................................... S-19

13. Optimized Geometries and Table of Energies: DABN Formation .............................. S-20

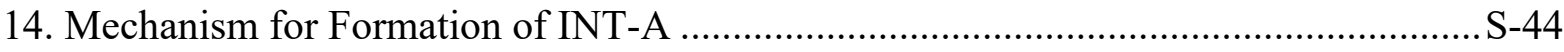

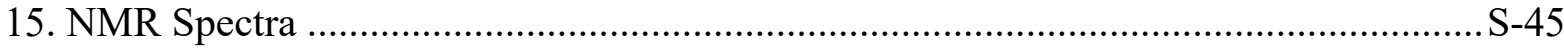

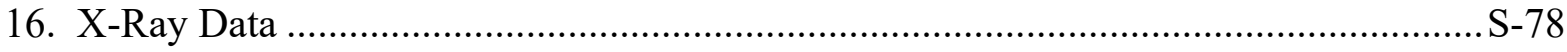

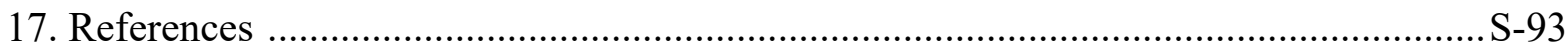




\section{General Experimental Information}

Solvents and Reagents: All solvents were of reagent-grade quality and purchased from commercial sources and utilized as received unless otherwise noted. $\mathrm{Pd}(\mathrm{OAc})_{2}$ was purchased from Strem and used as received. Tetrahydrofuran was dried by distillation over sodium/benzophenone prior to use. Acetonitrile and dichloromethane were distilled over calcium hydride. Unless otherwise noted, all reagents were purchased from commercial sources and used as received. 1,4Benzoquinone was purified by sublimation prior to use. The palladium sulfoxide complex was synthesized according to the published literature procedure. ${ }^{1}$

Chromatography: Flash column chromatography was carried out using Silicycle 230400 mesh silica gel. Thin-layer chromatography (TLC) was performed on Macherey Nagel precoated glass-backed TLC plates (SIL G/ UV254, $0.25 \mathrm{~mm}$ ) and visualized using a UV lamp $(254 \mathrm{~nm})$ followed by curcumin stain and potassium permanganate stain.

Nuclear Magnetic Resonance Spectroscopy: ${ }^{1} \mathrm{H}$ NMR, ${ }^{13} \mathrm{C}$ NMR, ${ }^{19} \mathrm{~F}$ and 2D NMR spectra were recorded on Varian Mercury 400, 500,600, MHz spectrometers or using a Bruker $400 \mathrm{MHz}$ spectrometer. ${ }^{11} \mathrm{~B}$ NMR were recorded using a Bruker 400 spectrometer at $128 \mathrm{MHz}$ and referenced to an external standard of $\mathrm{BF}_{3} \cdot \mathrm{OEt}_{2}(\delta=0 \mathrm{ppm}) .{ }^{1} \mathrm{H}$ NMR spectra chemical shifts $(\delta)$ are reported in parts per million $(\mathrm{ppm})$ referenced to residual protonated solvent peak $\left(\mathrm{CD}_{3} \mathrm{CN} \delta\right.$ $=1.94$, DMSO- $d_{6} \delta=2.50$, Acetone- $d_{6} \delta=2.05$ center line). Spectral data are reported as follows: chemical shift, multiplicity $(\mathrm{s}=$ singlet, $\mathrm{d}=$ doublet, $\mathrm{t}=$ triplet, $\mathrm{q}=$ quartet, $\mathrm{dd}=$ doublet of doublets, $\mathrm{dt}=$ doublet of triplets, $\mathrm{ddt}=$ doublet of doublet of triplets, $\mathrm{dtd}=$ doublet of triplet of doublets, $\mathrm{m}$ $=$ multiplet, $\mathrm{br}=$ broad, $\mathrm{h}=$ heptet, $\mathrm{dddd}=$ doublet of doublet of doublet of doublets, $\mathrm{qd}=$ quartet of doublets, $\mathrm{td}=$ triplet of doublets, $\mathrm{tt}=$ triplet of triplets $)$, coupling constant $(J)$ in hertz $(\mathrm{Hz})$, and integration. ${ }^{13} \mathrm{C}$ NMR spectra chemical shifts $(\delta)$ are reported in parts per million $(\mathrm{ppm})$ and were referenced to carbon resonances in the NMR solvent $\left(\mathrm{CD}_{3} \mathrm{CN} \delta=118.3\right.$, DMSO- $d_{6} \delta=39.5$, Acetone- $d_{6} \delta=206.26$ center line). Carbons exhibiting significant line broadening brought about by boron substituents were not found (quadrupolar relaxation).

High-resolution Mass Spectrometry: were obtained on a VG 70-250S (double focusing) mass spectrometer at $70 \mathrm{eV}$ or on an $\mathrm{ABI} / \mathrm{Sciex}$ Qstar mass spectrometer with ESI source, MS/MS, and accurate mass capabilities or on a JEOL AccuTOF-DART instrument.

FTIR and Melting Point Analysis: FTIR analysis was carried out on a Bruker Alpha Platinum ATP spectrometer. Melting point was measured utilizing a Mel-Temp capillary melting point apparatus. 


\section{Substrate Synthesis}

\section{$\operatorname{MIDA(allyl)~boronate~(1)~}$}

1 was synthesized according to the reported literature procedure. ${ }^{2}$ Purification
by silica gel column chromatography eluting with $20 \%$ acetone in hexanes ( $\mathrm{rf}$ $=0.25)$ afforded 1 as a white solid. ${ }^{1} \mathbf{H}$ NMR $\left(400 \mathrm{MHz}, \mathrm{CD}_{3} \mathrm{CN}\right) \delta 5.86(\mathrm{ddt}, J=17.0,10.2,7.6$ Hz, 1H), 5.00 (ddt, $J=17.1,2.4,1.5 \mathrm{~Hz}, 1 \mathrm{H}$ ), 4.92 (ddt, $J=10.2,2.4,1.2 \mathrm{~Hz}, 1 \mathrm{H}$ ), 3.94 (d, $J=$ $16.9 \mathrm{~Hz}, 2 \mathrm{H}), 3.75$ (d, $J=16.9 \mathrm{~Hz}, 2 \mathrm{H}), 2.89(\mathrm{~s}, 3 \mathrm{H}), 1.60(\mathrm{~d}, J=7.7 \mathrm{~Hz}, 2 \mathrm{H}) .{ }^{13} \mathbf{C}$ NMR $(126$ $\left.\mathrm{MHz}, \mathrm{CD}_{3} \mathrm{CN}\right) \delta 169.0,136.8,115.6,62.9,46.5 .{ }^{11} \mathbf{B}$ NMR $\left(128 \mathrm{MHz}, \mathrm{CD}_{3} \mathrm{CN}\right) \delta$ 12.2. FTIR (neat): $v=2961,1732,1340,1307,1254,1202,1095,1068,1018,987,956,901,854,808,670$, 629, 595, 499. HRMS (DART-TOF) m/z: $\left[\mathrm{M}+\mathrm{NH}_{4}\right]^{+}$Calcd for $\mathrm{C}_{8} \mathrm{H}_{16} \mathrm{BN}_{2} \mathrm{O}_{4}$ 215.1198; Found 215.1210. Melting Point: $115-116^{\circ} \mathrm{C}$.

\section{MIDA (E)-(3-acetoxyprop-1-en-1-yl)boronate (3)}

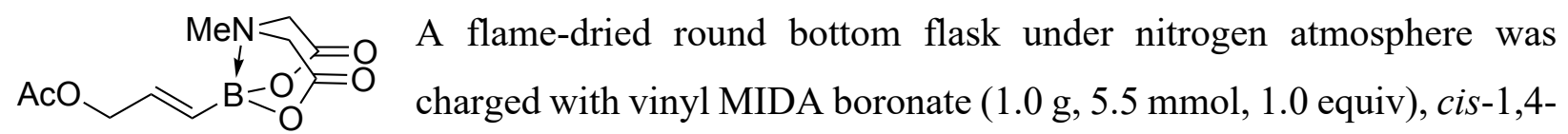
diacetoxy-2-butene ( $2.8 \mathrm{~g}, 13.8 \mathrm{mmol}, 2.5$ equiv) and Grubbs' second-generation catalyst ( $0.25 \mathrm{~g}$, $0.3 \mathrm{mmol}, 5 \mathrm{~mol} \%$ ). The contents of the flask were then suspended in freshy distilled and degassed dichloromethane $(140 \mathrm{ml}, 0.04 \mathrm{M})$. The round bottom flask was then fitted with a condenser brought to reflux utilizing an oil bath. After 24 hours, the reaction mixture was filtered through silica gel eluting with acetonitrile, concentrated in vacuo and the purified utilizing column chromatography eluting with 50 to $85 \%$ acetone in hexanes to afford the desired product as a white solid $(15 \%, 0.23 \mathrm{~g}) .{ }^{1} \mathbf{H}$ NMR $\left(500 \mathrm{MHz}, \mathrm{CD}_{3} \mathrm{CN}\right) \delta 6.10(\mathrm{dt}, J=17.9,4.9 \mathrm{~Hz}, 1 \mathrm{H}), 5.68(\mathrm{dt}, J=$ 17.9, $1.7 \mathrm{~Hz}, 1 \mathrm{H}), 4.59$ (dd, $J=4.9,1.7 \mathrm{~Hz}, 2 \mathrm{H}), 3.96$ (d, $J=17.0 \mathrm{~Hz}, 2 \mathrm{H}), 3.79$ (d, $J=16.9 \mathrm{~Hz}$, 2H), 2.76 (s, 3H), 2.03 (s, 3H). ${ }^{13} \mathbf{C}$ NMR (126 MHz, CD $\left.{ }_{3} \mathrm{CN}\right) \delta 171.3,169.2,139.8,66.3,62.3$, 47.6, 21.0. ${ }^{11} \mathbf{B}$ NMR (128 MHz, $\left.\mathrm{CD}_{3} \mathrm{CN}\right) \delta 3.3$. FTIR (neat): $v=2964,1748,1725,1650,1453$, 1434, 1382, 1340, 1307, 1294, 1268, 1232, 1196, 1151, 1112, 1085, 1072, 1023, 1003, 955, 895, 861, 630, 603. HRMS (ESI-TOF) m/z: $\left[\mathrm{M}+\mathrm{NH}_{4}\right]^{+}$Calcd for $\mathrm{C}_{10} \mathrm{H}_{18} \mathrm{BN}_{2} \mathrm{O}_{6}$ 273.1258; Found 273.1247. Melting Point: $80-83^{\circ} \mathrm{C}$. 


\section{Oxidative Rearrangement Optimization Studies}

A vial equipped with a Teflon-coated magnetic stir bar was charged with palladium complex, 1,4benzoquinone, 1 ( $0.3 \mathrm{mmol}, 1.0$ equiv), acetic acid and solvent $(0.1 \mathrm{M})$. The vial was capped and then placed into pre-heated oil bath set at the desired temperature. The reactions were quenched after 24 hours, by filtering through a silica plug using acetonitrile. Conversion and selectivity were then determined by ${ }^{1} \mathrm{H}$ NMR analysis of the crude reaction mixtures. Entries $2-8$ were performed using the pre-formed catalytic complex. In entry $1, \mathrm{Pd}(\mathrm{OAc})_{2}$ was mixed with phenylvinyl sulfoxide before adding the rest of the reagents in situ.

Table S-1. Optimization of the allylic oxidation conditions for the synthesis of $\mathbf{2 a}$.

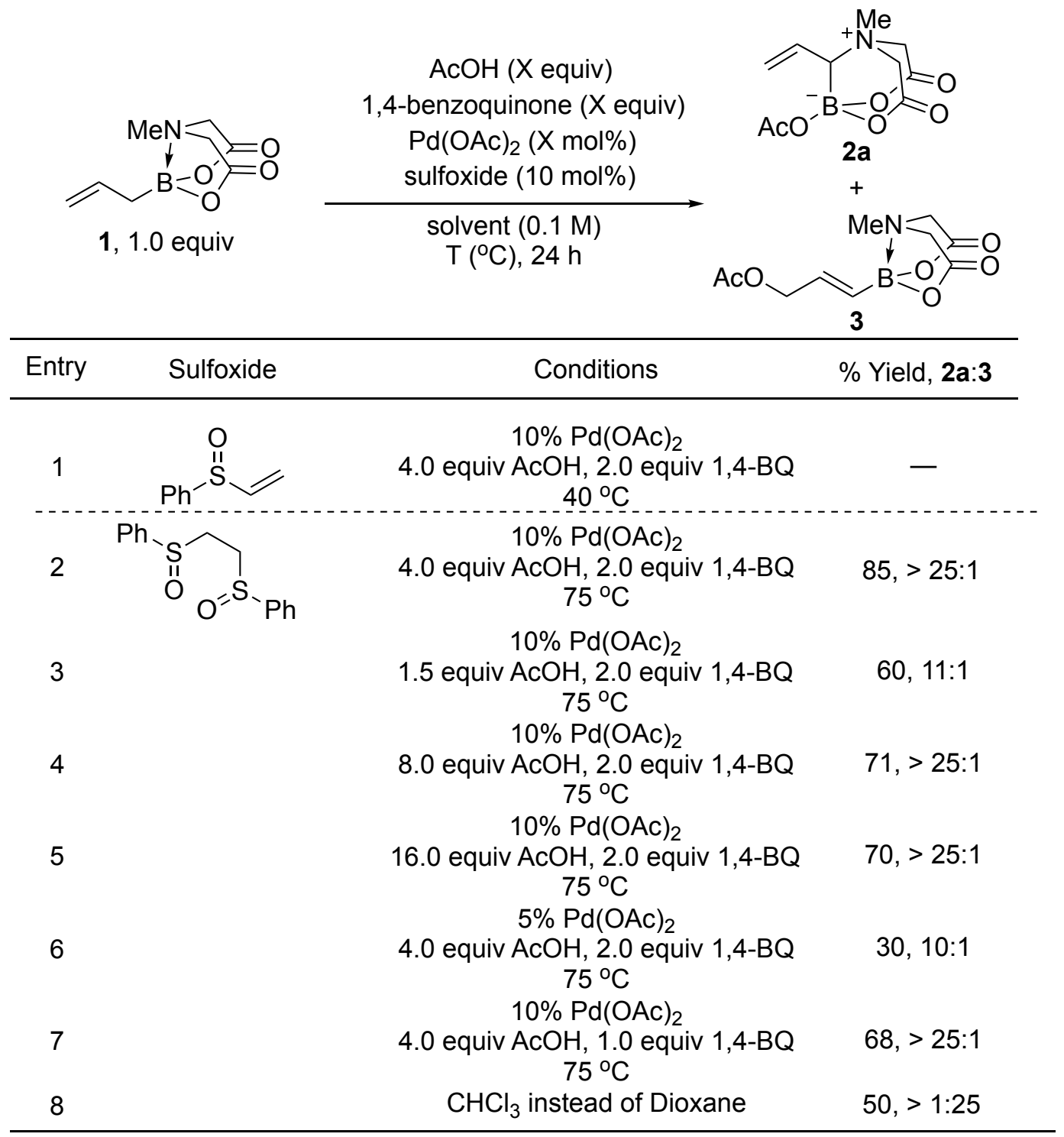




\section{Synthesis of DABN Boronates}

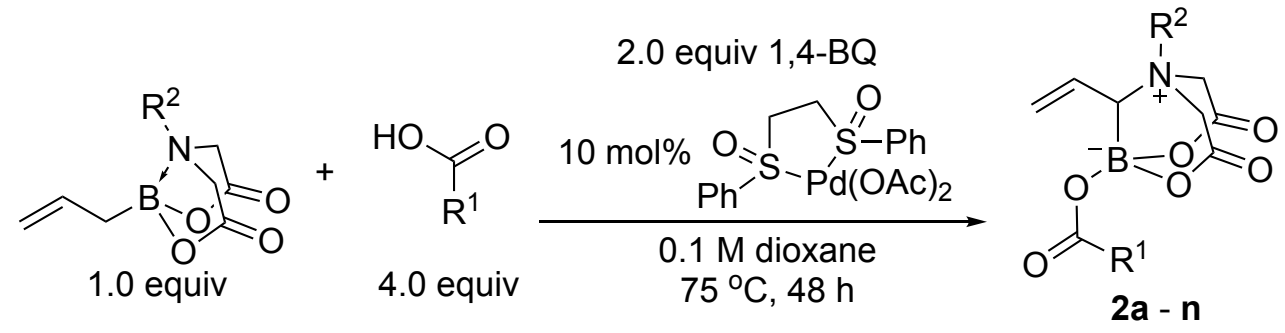

General Procedure for DABN Synthesis (GP1): A vial equipped with a Teflon-coated magnetic stir bar was charged with palladium complex $(0.03 \mathrm{mmol}, 10 \mathrm{~mol} \%), 1,4$-benzoquinone $(0.6 \mathrm{mmol}$, 2 equiv), MIDA(allyl) boronate ( $0.3 \mathrm{mmol}, 1.0$ equiv), carboxylic acid (1.2 mmol, 4 equiv) and dioxane $(3 \mathrm{ml}, 0.1 \mathrm{M})$. The vial was capped and then placed into a $75^{\circ} \mathrm{C}$ pre-heated oil bath. The reactions were monitored by TLC ( $40 \%$ acetonitrile in diethyl ether) and visualized by curcumin stain for the disappearance of the starting material. The reactions were typically complete between 24 and 48 hours, this was confirmed by crude ${ }^{1} \mathrm{H}$ NMR analysis of an aliquot from the reaction mixture. Upon completion, the reaction was concentrated and purified by silica gel column chromatography, eluting with either hexanes/acetone or acetonitrile/diethyl ether to afford the DABN boronate.

\section{1-Acetoxy-5-methyl-3,7-dioxo-9-vinyl-2,8-dioxa-5-aza-1-borabicyclo[3.3.1]nonan-5-ium-1-} uide (2a)

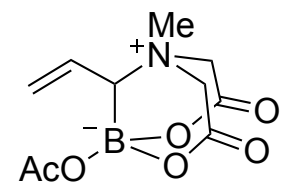

2a was synthesized according to GP1 and purified by silica gel column chromatography eluting with $40-60 \%$ acetone in hexanes with $1 \% \mathrm{Et}_{3} \mathrm{~N}$. The product was isolated as a white powder in 81\% yield (0.062 $\mathrm{g},>25: 1) .{ }^{1} \mathbf{H}$ NMR $\left(300 \mathrm{MHz}, \mathrm{CD}_{3} \mathrm{CN}\right) \delta 5.93-5.73(\mathrm{~m}, 1 \mathrm{H}), 5.58-5.43(\mathrm{~m}, 2 \mathrm{H}), 4.28-3.97(\mathrm{~m}, 4 \mathrm{H}), 3.88(\mathrm{~d}, J=$ $10.3 \mathrm{~Hz}, 1 \mathrm{H}), 3.00(\mathrm{~s}, 3 \mathrm{H}), 1.91(\mathrm{~s}, 3 \mathrm{H}) .{ }^{13} \mathrm{C}$ NMR $\left(126 \mathrm{MHz}, \mathrm{CD}_{3} \mathrm{CN}\right) \delta$ 171.8, 163.1, 163.0, 125.9, 118.3, 66.3, 61.0, 55.2, 23.0. ${ }^{11} \mathbf{B}$ NMR (96 MHz, $\left.\mathrm{CD}_{3} \mathrm{CN}\right) \delta$ 3.0. FTIR (neat): $v=2965$, 1744, 1721, 1650, 1453, 1382, 1289, 1112, 1071, 1010, 988, 951, 895, 859, 603, 541. HRMS (DART-TOF) m/z: $[\mathrm{M}+\mathrm{H}]^{+}$Calcd for $\mathrm{C}_{10} \mathrm{H}_{15} \mathrm{BNO}_{6}$ 256.0987; Found 256.0989. Melting Point: $247-250{ }^{\circ} \mathrm{C}$.

Compound 2a was successfully synthesized on a $5 \mathrm{mmol}$ scale as follows: In a dry round bottom flask equipped with Teflon-coated magnetic stir bar was added palladium complex $(0.250 \mathrm{~g}), 1,4-$ 
benzoquinone $(1.08 \mathrm{~g})$, acetic acid $(1.1 \mathrm{ml}), \mathbf{1}(0.985 \mathrm{~g})$ and 1,4-dioxane $(50 \mathrm{ml})$. The flask was sealed with a rubber septum and placed into pre-heated oil bath at $75^{\circ} \mathrm{C}$. After 24 hours, the reaction was concentrated and purified by silica gel chromatography to afford pure $\mathbf{2 a}(77 \%, 1.275$ g).

\section{1-(Benzoyloxy)-5-methyl-3,7-dioxo-9-vinyl-2,8-dioxa-5-aza-1-borabicyclo[3.3.1]nonan-5-}

ium-1-uide (2b)

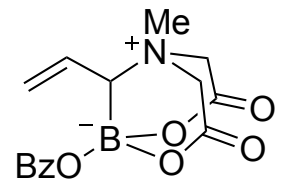

2b was synthesized according to GP1 and purified by silica gel chromatography eluting with $50-60 \%$ acetone in hexanes with $1 \% \mathrm{Et}_{3} \mathrm{~N}$. The product was isolated as a slightly beige powder in $90 \%$ yield $(0.086 \mathrm{~g}, 17: 1) .{ }^{1} \mathbf{H}$ NMR (400 $\left.\mathrm{MHz}, \mathrm{CD}_{3} \mathrm{CN}\right) \delta 8.01-7.96(\mathrm{~m}, 2 \mathrm{H}), 7.60-7.55(\mathrm{~m}, 1 \mathrm{H}), 7.48-7.43(\mathrm{~m}, 2 \mathrm{H}), 5.87$ (dt, $J=16.7$, $10.3 \mathrm{~Hz}, 1 \mathrm{H}), 5.53-5.40(\mathrm{~m}, 2 \mathrm{H}), 4.32-4.02(\mathrm{~m}, 5 \mathrm{H}), 3.05(\mathrm{~s}, 3 \mathrm{H}) .{ }^{13} \mathbf{C} \mathbf{~ N M R}\left(126 \mathrm{MHz}, \mathrm{CD}_{3} \mathrm{CN}\right)$ $\delta$ 166.9, 163.2, 163.0, 133.6, 133.3, 130.4, 129.3, 128.7, 126.1, 66.5, 61.1, 55.4. ${ }^{11}$ B NMR (128 $\mathrm{MHz}, \mathrm{CD}_{3} \mathrm{CN}$ ) $\delta$ 3.6. FTIR (neat): $v=3007,2962,1740,1701,1514,1451,1382,1356,1316$, 1296, 1245, 1136, 1069, 1011, 984, 971, 956, 941, 897, 861, 825, 808, 714, 690, 604, 579, 530. HRMS (ESI-TOF) m/z: $[\mathrm{M}+\mathrm{H}]^{+}$Calcd for $\mathrm{C}_{15} \mathrm{H}_{17} \mathrm{BNO}_{6}$ 318.1143; Found 318.1147. Melting Point: $245-247^{\circ} \mathrm{C}$.

\section{5-Methyl-1-((2-methylbenzoyl)oxy)-3,7-dioxo-9-vinyl-2,8-dioxa-5-aza-1-}

\section{borabicyclo[3.3.1]nonan-5-ium-1-uide (2c)}

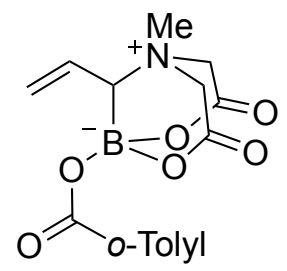

$\mathbf{2 c}$ was synthesized according to GP1 and purified by silica gel chromatography eluting with $30-60 \%$ acetone in hexanes with $1 \% \mathrm{Et}_{3} \mathrm{~N}$. The product was isolated as a white powder in $60 \%$ yield $(0.060 \mathrm{~g}, 11: 1) .{ }^{1} \mathbf{H}$ NMR $(500 \mathrm{MHz}$, Acetone- $\left.d_{6}\right) \delta 7.87(\mathrm{dd}, J=8.0,1.5 \mathrm{~Hz}, 1 \mathrm{H}), 7.37(\mathrm{td}, J=7.5,1.5 \mathrm{~Hz}, 1 \mathrm{H}), 7.26$ $-7.22(\mathrm{~m}, 2 \mathrm{H}), 6.02(\mathrm{dt}, J=16.7,10.3 \mathrm{~Hz}, 1 \mathrm{H}), 5.52(\mathrm{ddd}, J=16.7,1.9,0.6 \mathrm{~Hz}, 1 \mathrm{H}), 5.45(\mathrm{dd}, J$ $=10.2,1.9 \mathrm{~Hz}, 1 \mathrm{H}), 4.61-4.43(\mathrm{~m}, 4 \mathrm{H}), 4.18(\mathrm{~d}, J=10.4 \mathrm{~Hz}, 1 \mathrm{H}), 3.40(\mathrm{~s}, 3 \mathrm{H}), 2.55(\mathrm{~s}, 3 \mathrm{H}) .{ }^{13} \mathrm{C}$ NMR (126 MHz, Acetone- $\left.d_{6}\right) \delta 168.0,163.2,162.9,140.3,133.4,132.2,132.1,131.50,129.6$, 126.5, 125.1, 125.1, 66.5, 61.0, 55.2, 21.9. ${ }^{11} \mathbf{B}$ NMR $\left(128 \mathrm{MHz}, \mathrm{CD}_{3} \mathrm{CN}\right) \delta 3.6$. FTIR (neat): $v=$ 2960, 1742, 1697, 1296, 1109, 1072, 1009, 984, 954, 737. HRMS (ESI-TOF) m/z: [M+H] Calcd for $\mathrm{C}_{16} \mathrm{H}_{19} \mathrm{BNO}_{6} 332.1300$; Found 332.1308. Melting Point: $256-259^{\circ} \mathrm{C}$. 


\section{5-Methyl-1-((4-methylbenzoyl)oxy)-3,7-dioxo-9-vinyl-2,8-dioxa-5-aza-1-}

\section{borabicyclo[3.3.1]nonan-5-ium-1-uide (2d)}

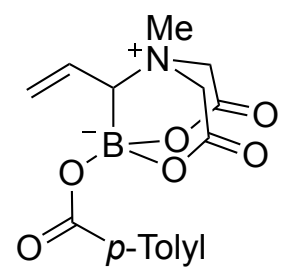

2d was synthesized according to GP1 and purified by silica gel chromatography eluting with $25-50 \%$ acetone in hexanes with $1 \% \mathrm{Et}_{3} \mathrm{~N}$. The product was isolated as a white solid in 73\% yield (0.073 g, 20:1). ${ }^{1} \mathbf{H}$ NMR (400 MHz, $\left.\mathrm{CD}_{3} \mathrm{CN}\right) \delta 7.87(\mathrm{~d}, J=8.2 \mathrm{~Hz}, 1 \mathrm{H}), 7.26(\mathrm{~d}, J=7.9 \mathrm{~Hz}, 1 \mathrm{H}), 5.86(\mathrm{dt}, J=16.8$, $10.3 \mathrm{~Hz}, 1 \mathrm{H}), 5.56-5.35(\mathrm{~m}, 1 \mathrm{H}), 4.29-4.03(\mathrm{~m}, 5 \mathrm{H}), 3.04(\mathrm{~s}, 3 \mathrm{H}), 2.39$ (s, 3H). ${ }^{13} \mathbf{C}$ NMR (126 $\left.\mathrm{MHz}, \mathrm{CD}_{3} \mathrm{CN}\right) \delta 166.9,163.2,162.9,144.3,130.6,130.5,129.9,128.7,126.0,66.4,61.0,55.3$, 21.6. ${ }^{11}$ B NMR (128 MHz, CD $\left.{ }_{3} \mathrm{CN}\right) \delta$ 3.2. FTIR (neat): $v=3005,2961,1698,1612,1459,1380$, $1355,1290,1245,1176,1136,1068,1010,984,970,955,897,837,781,758,706,670,586,531$, 475. HRMS (ESI-TOF) m/z: [M+H] ${ }^{+}$Calcd for $\mathrm{C}_{16} \mathrm{H}_{19} \mathrm{BNO}_{6} 332.1300$; Found 332.1306. Melting Point: $282-284^{\circ} \mathrm{C}$.

\section{1-((2-Chloro-4-methoxybenzoyl)oxy)-5-methyl-3,7-dioxo-9-vinyl-2,8-dioxa-5-aza-1-}

\section{borabicyclo[3.3.1]nonan-5-ium-1-uide (2e)}

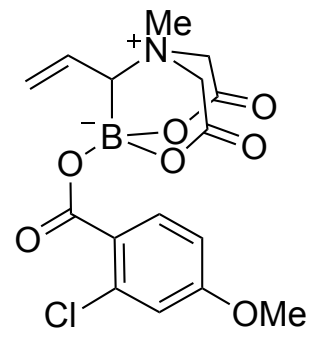

2e was synthesized according to GP1 and purified by silica gel chromatography eluting with $25-50 \%$ acetone in hexanes with $1 \% \mathrm{Et}_{3} \mathrm{~N}$. The product was isolated as a white film in $73 \%$ yield $(0.084 \mathrm{~g}, 10: 1) .{ }^{1} \mathbf{H}$ NMR $\left(400 \mathrm{MHz}, \mathrm{CD}_{3} \mathrm{CN}\right) \delta 7.84(\mathrm{~d}, J=8.8 \mathrm{~Hz}, 1 \mathrm{H}), 7.02(\mathrm{~d}, J=2.5 \mathrm{~Hz}, 1 \mathrm{H}), 6.89$

$4.26-4.02(\mathrm{~m}, 5 \mathrm{H}), 3.83(\mathrm{~s}, 3 \mathrm{H}), 3.04(\mathrm{~s}, 3 \mathrm{H}) .{ }^{13} \mathrm{C}$ NMR (126 MHz, CD $\left.{ }_{3} \mathrm{CN}\right) \delta 165.3,163.3$, $163.1,162.9,135.7,134.2,128.6,126.1,124.7,117.2,113.6,66.4,61.0,56.6 .{ }^{11}$ B NMR (128 $\mathrm{MHz} \mathrm{CD}{ }_{3} \mathrm{CN}$ ) $\delta$ 3.5. FTIR (neat): $v=1690,1599,1316,1292,1268,1235,1147,1073,1026$, 1002, 956, 938, 897, 861, 777, 588. HRMS (ESI-TOF) m/z: $[\mathrm{M}+\mathrm{H}]^{+}$Calcd for $\mathrm{C}_{16} \mathrm{H}_{18} \mathrm{BClNO}_{7}$ 382.0859; Found 382.0858. Melting Point: $251-253^{\circ} \mathrm{C}$ 


\section{5-Methyl-3,7-dioxo-1-(propionyloxy)-9-vinyl-2,8-dioxa-5-aza-1-borabicyclo[3.3.1]nonan-5-}

ium-1-uide (2f)

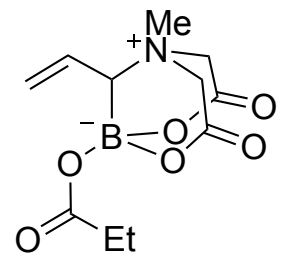

2f was synthesized according to GP1 and purified by silica gel chromatography eluting with $50 \%$ acetone in hexanes with $1 \% \mathrm{Et}_{3} \mathrm{~N}$. The product was isolated as a white solid in 78\% yield (0.063 g, > 25:1). ${ }^{1} \mathbf{H}$ NMR $\left(400 \mathrm{MHz}, \mathrm{CD}_{3} \mathrm{CN}\right) \delta$ $5.82(\mathrm{dt}, J=16.3,10.5 \mathrm{~Hz}, 1 \mathrm{H}), 5.50-5.43(\mathrm{~m}, 2 \mathrm{H}), 4.25-3.97(\mathrm{~m}, 4 \mathrm{H}), 3.00$ $(\mathrm{s}, 3 \mathrm{H}), 3.91(\mathrm{~d}, J=10.0 \mathrm{~Hz}, 1 \mathrm{H}), 2.21(\mathrm{qd}, J=7.5,4.0 \mathrm{~Hz}, 2 \mathrm{H}), 1.01(\mathrm{t}, J=7.5 \mathrm{~Hz}, 3 \mathrm{H}) .{ }^{13} \mathbf{C}$ NMR $\left(126 \mathrm{MHz}, \mathrm{CD}_{3} \mathrm{CN}\right) \delta \delta 175.2,163.1,163.0,128.7,125.8,66.3,62.3,60.9,55.3,29.6,9.6$. ${ }^{11}$ B NMR (128 MHz, CD 3 CN) $\delta 3.1$. FTIR (neat): $v=2965,1735,1696,1346,1240,1070,1039$, 1018, 987, 973, 956, 937, 906, 840, 807, 573. HRMS (ESI-TOF) m/z: $[\mathrm{M}+\mathrm{H}]^{+}$Calcd for $\mathrm{C}_{11} \mathrm{H}_{17} \mathrm{BNO}_{6} 270.1143$; Found 270.1146. Melting Point: $243-245^{\circ} \mathrm{C}$.

\section{5-Methyl-1-((3-methylbutanoyl)oxy)-3,7-dioxo-9-vinyl-2,8-dioxa-5-aza-1-}

\section{borabicyclo[3.3.1]nonan-5-ium-1-uide (2g)}

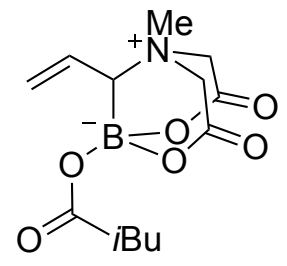

2g was synthesized according to GP1 and purified by silica gel chromatography eluting with $20-30 \%$ acetone in hexanes with $1 \% \mathrm{Et}_{3} \mathrm{~N}$. The product was isolated as a white solid in $60 \%$ yield $(0.053 \mathrm{~g},>25: 1) .{ }^{1} \mathbf{H}$ NMR $(500 \mathrm{MHz}$, $\left.\mathrm{CD}_{3} \mathrm{CN}\right) \delta 5.83-5.75(\mathrm{~m}, 1 \mathrm{H}), 5.48-5.47(\mathrm{~m}, 1 \mathrm{H}), 5.46-5.44(\mathrm{~m}, 1 \mathrm{H}), 4.20$ $-3.98(\mathrm{~m}, 4 \mathrm{H}), 3.90(\mathrm{~d}, J=10.5 \mathrm{~Hz}, 1 \mathrm{H}), 2.97(\mathrm{~s}, 3 \mathrm{H}), 2.10-2.01(\mathrm{~m}, 2 \mathrm{H}), 1.99-1.94(\mathrm{~m}, 1 \mathrm{H}), \mathrm{f}$ $0.88(\mathrm{dd}, J=6.6,2.9 \mathrm{~Hz}, 6 \mathrm{H}) .{ }^{13} \mathbf{C}$ NMR $\left(126 \mathrm{MHz}, \mathrm{CD}_{3} \mathrm{CN}\right) \delta 173.8,163.1,160.0,128.7,126.1$, 66.3, 61.0, 55.2, 45.65, 26.4, 22.6. ${ }^{11} \mathbf{B}$ NMR (126 MHz, CD $\left.{ }_{3} \mathrm{CN}\right) \delta$ 3.6. FTIR (neat): $v=2960$, 1697, 1340, 1300, 1237, 1210, 1068, 1032, 1010, 987, 971, 953, 912, 854. HRMS (ESI-TOF) m/z: $[\mathrm{M}+\mathrm{H}]^{+}$Calcd for $\mathrm{C}_{13} \mathrm{H}_{21} \mathrm{BNO}_{6} 298.1456$; Found 298.1458. Melting Point: $271-273{ }^{\circ} \mathrm{C}$.

\section{1-(Decanoyloxy)-5-methyl-3,7-dioxo-9-vinyl-2,8-dioxa-5-aza-1-borabicyclo[3.3.1]nonan-5-} ium-1-uide (2h)

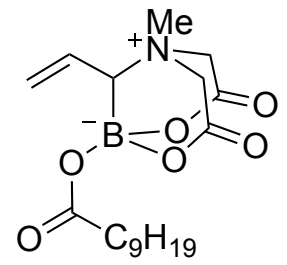

2h was synthesized according to GP1 and purified by silica gel chromatography eluting with $15 \%$ acetonitrile in diethyl ether $1 \% \mathrm{Et}_{3} \mathrm{~N}$. The product was isolated as a light brown solid in 64\% yield $(0.071 \mathrm{~g},>25: 1) .{ }^{1} \mathbf{H}$ NMR (400 MHz, $\left.\mathrm{CD}_{3} \mathrm{CN}\right) \delta 5.86-5.76(\mathrm{~m}, 1 \mathrm{H}), 5.49(\mathrm{~s}, 1 \mathrm{H}), 5.47-5.44(\mathrm{~m}, 2 \mathrm{H}), 4.23-4.00$ (m, 4H), $3.91(\mathrm{~d}, J=10.3 \mathrm{~Hz}, 1 \mathrm{H}), 3.00(\mathrm{~s}, 3 \mathrm{H}), 2.19$ (td, $J=7.3,1.9 \mathrm{~Hz}, 2 \mathrm{H}), 1.51$ (p, $J=6.3,5.8$ $\mathrm{Hz}, 3 \mathrm{H}), 1.33-1.21(\mathrm{~m}, 12 \mathrm{H}), 0.90-0.87(\mathrm{~m}, 3 \mathrm{H}) .{ }^{13} \mathbf{C} \mathbf{N M R}\left(126 \mathrm{MHz}, \mathrm{CD}_{3} \mathrm{CN}\right) 174.5,163.1$, 
163.0, 128.7, 125.9, 66.3, 61.0, 55.3, 36.4, 32.6, 30.2, 30.0, 30.0, 29.8, 25.9, 23.3, 14.4. ${ }^{11} \mathbf{B}$ NMR $\left(128 \mathrm{MHz}, \mathrm{CD}_{3} \mathrm{CN}\right) \delta 3.1$. FTIR (neat): $v=2958,2923,2853,1741,1712,1460,1379,1350$, 1319, 1236, 1212, 1147, 1113, 1070, 1033, 1012, 987, 970, 949, 918, 856, 835, 809, 708, 598, 576, 531. HRMS (DART-TOF) m/z: $[\mathrm{M}+\mathrm{H}]^{+}$Calcd for $\mathrm{C}_{18} \mathrm{H}_{31} \mathrm{BNO}_{6}$ 368.2239; Found 368.225. Melting Point: $260-263^{\circ} \mathrm{C}$.

\section{1-Acetoxy-5-dodecyl-3,7-dioxo-9-vinyl-2,8-dioxa-5-aza-1-borabicyclo[3.3.1]nonan-5-ium-1-} uide (2i)

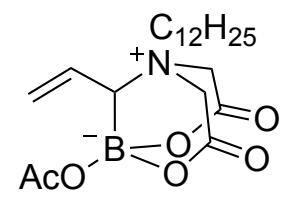

$\mathbf{2 i}$ was synthesized according to GP1 and purified by silica gel chromatography eluting with $10-20 \%$ acetonitrile in diethyl ether with $1 \% \mathrm{Et}_{3} \mathrm{~N}$. The product was isolated as a white solid in $65 \%$ yield $(0.080 \mathrm{~g}, 10: 1) .{ }^{1} \mathbf{H}$ NMR $(500 \mathrm{MHz}$, $\left.\mathrm{CD}_{3} \mathrm{CN}\right) \delta 5.80(\mathrm{dt}, J=16.5,10.5 \mathrm{~Hz}, 1 \mathrm{H}), 5.47-5.41(\mathrm{~m}, 2 \mathrm{H}), 4.18-4.01(\mathrm{~m}, 4 \mathrm{H}), 3.89(\mathrm{~d}, J=$ $10.5 \mathrm{~Hz}, 1 \mathrm{H}), 3.31(\mathrm{td}, J=12.7,4.8 \mathrm{~Hz}, 1 \mathrm{H}), 3.11(\mathrm{td}, J=12.7,4.6 \mathrm{~Hz}, 1 \mathrm{H}), 1.91(\mathrm{~s}, 3 \mathrm{H}), 1.81-$ $1.64(\mathrm{~m}, 2 \mathrm{H}), 1.35-1.21(\mathrm{~m}, 18 \mathrm{H}), 0.93-0.75(\mathrm{~m}, 3 \mathrm{H}) .{ }^{13} \mathrm{C} \mathrm{NMR}\left(126 \mathrm{MHz}, \mathrm{CD}_{3} \mathrm{CN}\right) \delta 171.8$, 163.2, 163.1, 128.8, 128.8, 124.8, 68.5, 62.9, 59.8, 32.6, 30.3, 30.3, 30.1, 30.0, 29.9, 29.6, 26.7, 23.4, 23.2, 22.0, 14.4. ${ }^{11} \mathbf{B}$ NMR (128 MHz, CD $\left.{ }_{3} \mathrm{CN}\right) \delta 3.0$. FTIR (neat): $v=3006,2960,2922$, 2849, 1721, 1691, 1474, 1359, 1293, 1224, 1089, 1070, 1057, 1034, 990, 968, 943, 906, 877, 858, 835, 665, 584, 560, 536. HRMS (DART-TOF) m/z: $[\mathrm{M}+\mathrm{H}]^{+}$Calcd for $\mathrm{C}_{21} \mathrm{H}_{37} \mathrm{BNO}_{6} 410.2708$; Found 410.2719. Melting Point: $140-142{ }^{\circ} \mathrm{C}$.

\section{5-Methyl-3,7-dioxo-1-(2-phenylacetoxy)-9-vinyl-2,8-dioxa-5-aza-1-borabicyclo[3.3.1]nonan-}

\section{5-ium-1-uide (2j)}

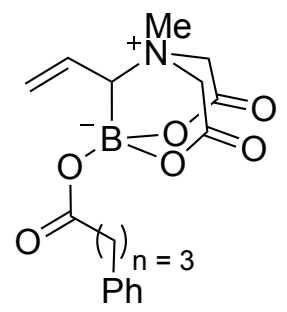

$\mathbf{2} \mathbf{j}$ was synthesized according to GP1 and purified by silica gel chromatography eluting with $25-50 \%$ acetone in hexanes with $1 \% \mathrm{Et}_{3} \mathrm{~N}$. The product was isolated as a white solid in $65 \%$ yield $(0.070 \mathrm{~g}, 22: 1) .{ }^{1} \mathbf{H}$ NMR $(500 \mathrm{MHz}$, $\left.\mathrm{CD}_{3} \mathrm{CN}\right) \delta 7.31-7.27(\mathrm{~m}, 2 \mathrm{H}), 7.22-7.16(\mathrm{~m}, 3 \mathrm{H}), 5.87-5.78(\mathrm{~m}, 1 \mathrm{H}), 5.51$ $-5.47(\mathrm{~m}, 2 \mathrm{H}), 4.25-4.01(\mathrm{~m}, 4 \mathrm{H}), 3.93(\mathrm{~d}, \mathrm{~J}=10.5 \mathrm{~Hz}, 1 \mathrm{H}), 3.00(\mathrm{~s}, 3 \mathrm{H}), 2.62$ $-2.58(\mathrm{~m}, 2 \mathrm{H}), 2.21(\mathrm{td}, \mathrm{J}=7.2,3.5 \mathrm{~Hz}, 2 \mathrm{H}), 1.85-1.78(\mathrm{~m}, 2 \mathrm{H}) .{ }^{13} \mathbf{C} \mathbf{N M R}\left(126 \mathrm{MHz}, \mathrm{CD}_{3} \mathrm{CN}\right)$ $\delta 174.2,163.2,163.1,143.0,129.3,129.2,128.6,128.6,126.7,126.0,66.2,60.9,55.2,35.7,35.5$, 27.7. ${ }^{11} \mathbf{B}$ NMR $\left(128 \mathrm{MHz}, \mathrm{CD}_{3} \mathrm{CN}\right) \delta$ 3.2. FTIR (neat): $v=3006,2962,2927,1740,1697,1454$, $1378,1341,1307,1271,1233,1145,1069,1010,986,969,947,917,852,834,808,744,698$, 
666, 598, 566, 531, 489. HRMS (ESI-TOF) m/z: $[\mathrm{M}+\mathrm{H}]^{+}$Calcd for $\mathrm{C}_{18} \mathrm{H}_{23} \mathrm{BNO}_{6} 360.1613$; Found 360.1618. Melting Point: $200-203{ }^{\circ} \mathrm{C}$.

\section{1-((4-Fluorobenzoyl)oxy)-5-methyl-3,7-dioxo-9-vinyl-2,8-dioxa-5-aza-1-}

\section{borabicyclo[3.3.1]nonan-5-ium-1-uide (2k)}

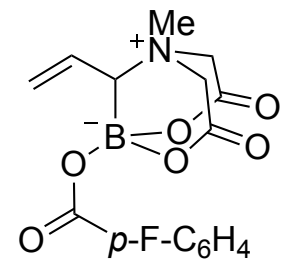

2k was synthesized according to GP1 and purified by silica gel chromatography eluting with $40-80 \%$ acetone in hexanes with $1 \% \mathrm{Et}_{3} \mathrm{~N}$. The product was isolated as a white solid in 70\% yield $(0.070 \mathrm{~g}, 16: 1) .{ }^{1} \mathbf{H}$ NMR $(500 \mathrm{MHz}$, $\left.\mathrm{CD}_{3} \mathrm{CN}\right) \delta 8.05-8.00(\mathrm{~m}, 2 \mathrm{H}), 7.20-7.14(\mathrm{~m}, 2 \mathrm{H}), 5.87(\mathrm{dt}, J=16.7,10.3 \mathrm{~Hz}$, 1H), $5.52-5.37(\mathrm{~m}, 1 \mathrm{H}), 4.31-4.06(\mathrm{~m}, 4 \mathrm{H}), 4.04(\mathrm{~d}, J=10.3 \mathrm{~Hz}, 1 \mathrm{H}), 3.04(\mathrm{~s}, 3 \mathrm{H}) .{ }^{13} \mathbf{C}$ NMR $\left(126 \mathrm{MHz}, \mathrm{CD}_{3} \mathrm{CN}\right) \delta 166.6(\mathrm{C}-\mathrm{F}, 1 \mathrm{JC}-\mathrm{F}=184.3 \mathrm{~Hz}), 165.9,163.1,162.9 .133 .11(\mathrm{C}-\mathrm{F}, 3 \mathrm{JC}-\mathrm{F}=$ 9.3 Hz), 129.76 (CF, 4JC-F = 2.7 Hz), 128.6, 126.1, $116.17(\mathrm{C}-\mathrm{F}, 2 \mathrm{JC}-\mathrm{F}=22.2 \mathrm{~Hz}), 66.4,61.0$, 55.3. ${ }^{11} \mathbf{B}$ NMR $\left(128 \mathrm{MHz}, \mathrm{CD}_{3} \mathrm{CN}\right) \delta 3.6 .{ }^{9} \mathbf{F}$ NMR $\left(377 \mathrm{MHz}, \mathrm{CD}_{3} \mathrm{CN}\right) \delta-108.91--109.12(\mathrm{~m})$ FTIR (neat): $v=3007,2963,2926,1734,1701,1605,1507,1459,1381,1356,1293,1238,1135$, 1068, 1011, 984, 970, 956, 939, 897, 850, 772, 708 ,688, 668, 644, 583, 532, 501. HRMS (ESITOF) m/z: $[\mathrm{M}+\mathrm{H}]^{+}$Calcd for $\mathrm{C}_{15} \mathrm{H}_{16} \mathrm{BFNO}_{6}$ 336.1049; Found 336.1058. Melting Point: 285 - 287 ${ }^{\circ} \mathrm{C}$.

\section{1-((4-Methoxybenzoyl)oxy)-5-methyl-3,7-dioxo-9-vinyl-2,8-dioxa-5-aza-1-}

\section{borabicyclo[3.3.1]nonan-5-ium-1-uide (2I)}

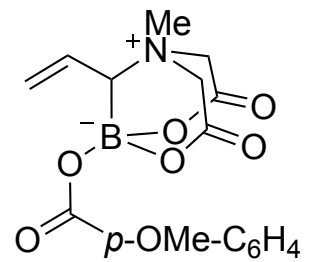

2l was synthesized according to GP1 and purified by silica gel chromatography eluting with $40-60 \%$ acetone in hexanes with $1 \% \mathrm{Et}_{3} \mathrm{~N}$. The product was isolated as a white solid in 33\% yield (0.034 g, 23:1). ${ }^{1} \mathbf{H}$ NMR (500 MHz, $\left.\mathrm{CD}_{3} \mathrm{CN}\right) \delta 7.95-7.92(\mathrm{~m}, 2 \mathrm{H}), 6.97-6.93(\mathrm{~m}, 2 \mathrm{H}), 5.86(\mathrm{dt}, J=16.7,10.3$ $\mathrm{Hz}, 1 \mathrm{H}), 5.51-5.409 \mathrm{~m}, 2 \mathrm{H}), 4.30-4.03(\mathrm{~m}, 5 \mathrm{H}), 3.84$ (s, 3H), 3.04 (s, 3H). ${ }^{13} \mathrm{C} \mathrm{NMR}(126 \mathrm{MHz}$, $\left.\mathrm{CD}_{3} \mathrm{CN}\right) \delta 166.6,164.1,163.2,163.0,132.4,128.7,126.0,125.7,114.5,66.4,61.0,56.2,55.3 .{ }^{11} \mathbf{B}$ NMR $\left(128 \mathrm{MHz}, \mathrm{CD}_{3} \mathrm{CN}\right) \delta$ 3.6. FTIR (neat): $v=1692,1672,1605,1323,1307,1296,1256$, 1167, 1138, 1072, 1034, 997, 981, 957, 936, 844, 827, 776, 703, 580. HRMS (ESI-TOF) m/z: $[\mathrm{M}+\mathrm{H}]^{+}$Calcd for $\mathrm{C}_{16} \mathrm{H}_{19} \mathrm{BNO}_{7} 348.1249$; Found 348.1255. Melting Point: $229-232{ }^{\circ} \mathrm{C}$. 


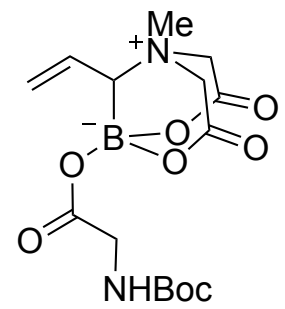

2m was synthesized according to GP1 and purified by silica gel chromatography eluting with $30 \%$ acetonitrile in diethyl ether with $1 \% \mathrm{Et}_{3} \mathrm{~N}$. The product was isolated as a white solid in $70 \%$ yield $(0.078 \mathrm{~g}, 11: 1) .{ }^{1} \mathbf{H}$ NMR $(500 \mathrm{MHz}$, $\left.\mathrm{CD}_{3} \mathrm{CN}\right) \delta 5.82(\mathrm{dtd}, J=16.9,10.4,6.7 \mathrm{~Hz}, 1 \mathrm{H}), 5.51-5.54(\mathrm{~m}, 3 \mathrm{H}), 4.27-$ $4.12(\mathrm{~m}, 1 \mathrm{H}), 4.08-4.01(\mathrm{~m}, 1 \mathrm{H}), 3.88(\mathrm{~d}, J=10.3 \mathrm{~Hz}, 1 \mathrm{H}), 3.66$ (qd, $J=18.0$, $6.2 \mathrm{~Hz}, 1 \mathrm{H}), 3.01$ (s, 3H), 1.4 (s, 9H). ${ }^{13} \mathbf{C}$ NMR $\left(126 \mathrm{MHz}, \mathrm{CD}_{3} \mathrm{CN}\right) \delta$ 171.9, 171.2, 163.1, 163.0, 163.0, 162.9, 156.8, 128.6, 128.3, 126.1, 125.9, 79.6, 66.3, 66.3, 60.9, 60.9, 55.2, 55.2, 43.8, 30.6, 28.5, 23.0. ${ }^{11} \mathbf{B}$ NMR $\left(128 \mathrm{MHz}, \mathrm{CD}_{3} \mathrm{CN}\right) \delta 3.1$. HRMS (ESI-TOF) m/z: $\left[\mathrm{M}+\mathrm{NH}_{4}\right]^{+}$Calcd for $\mathrm{C}_{15} \mathrm{H}_{27} \mathrm{BN}_{3} \mathrm{O}_{8}$ 388.1886; Found 388.1889. FTIR (neat): $v=3425,2960,1697,1514,1366,1272$, 1230, 1157, 1077, 1034, 1011, 987, 973, 962, 944, 914, 849, 574. Melting Point: $219-221^{\circ} \mathrm{C}$.

\section{1-(Acryloyloxy)-5-methyl-3,7-dioxo-9-vinyl-2,8-dioxa-5-aza-1-borabicyclo[3.3.1]nonan-5- ium-1-uide (2n)}

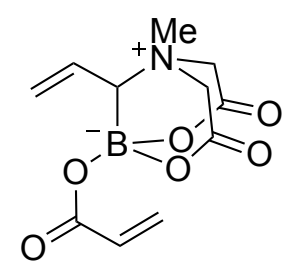

2n was synthesized according to GP1 and purified by silica gel chromatography eluting with $25-50 \%$ acetone in hexanes with $1 \% \mathrm{Et}_{3} \mathrm{~N}$. The product was isolated as a pale yellow solid in $67 \%$ yield $(0.054 \mathrm{~g},>25: 1) .{ }^{1} \mathbf{H}$ NMR $\left(\mathrm{CD}_{3} \mathrm{CN}\right) \delta 6.26(\mathrm{dd}, J=17.3,1.8 \mathrm{~Hz}, 1 \mathrm{H}), 6.06(\mathrm{dd}, J=17.3,10.3 \mathrm{~Hz}$, 1H), $5.88-5.80$ (m, 1H), 5.77 (dd, $J=10.2,1.8 \mathrm{~Hz}, 1 \mathrm{H}), 4.26-4.00$ (m, 4H), 3.94 (d, $J=10.4$ $\mathrm{Hz}, 1 \mathrm{H}), 3.01$ (s, 3H). ${ }^{13} \mathbf{C}$ NMR $\left(126 \mathrm{MHz}, \mathrm{CD}_{3} \mathrm{CN}\right) \delta 166.6,163.1,162.9,131.9,130.3,128.6$, 126.0, 66.4, 61.0, 55.3. ${ }^{11} \mathbf{B}$ NMR $\left(128 \mathrm{MHz}, \mathrm{CD}_{3} \mathrm{CN}\right) \delta$ 3.3. FTIR (neat): $v=1924,1694,1307$, 1240, 1076, 1033, 1014, 986, 953, 941, 905. HRMS (ESI-TOF) m/z: [M+H] $]^{+}$Calcd for $\mathrm{C}_{11} \mathrm{H}_{15} \mathrm{BNO}_{6} 268.0987$; Found 268.0990. Melting Point: 277 - $279{ }^{\circ} \mathrm{C}$. 


\section{Functionalization of DABN Boronates}

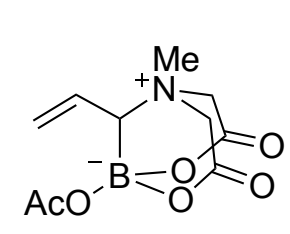

2a, 1.0 equiv

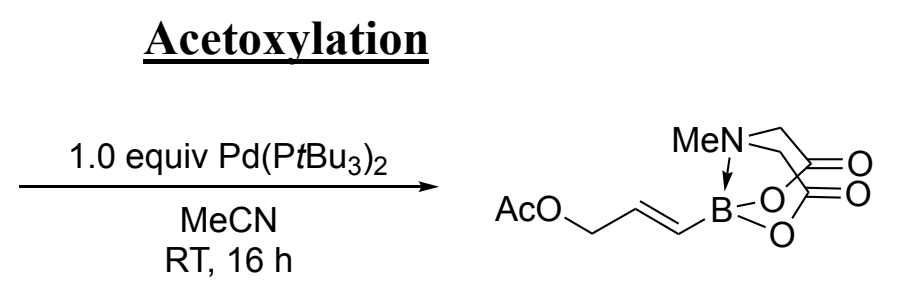

3, $47 \%$

In a nitrogen-filled glovebox, a 2-dram vial equipped with teflon-coated stir bar was charged with 2a $\left(0.010 \mathrm{~g}, 0.4 \mathrm{mmol}, 1.0\right.$ equiv) and $\mathrm{Pd}\left(\mathrm{P}^{t} \mathrm{Bu}_{3}\right)_{2}(34 \mathrm{mg}, 0.4 \mathrm{mmol}$, 1.0 equiv) and dry $\mathrm{CD}_{3} \mathrm{CN}(0.5 \mathrm{ml})$. The reaction was stirred inside the glovebox for 16 hours at room temperature. At which time an aliquot was taken directly from the reaction mixture and analyzed by ${ }^{1} \mathrm{H}$ NMR. ${ }^{1} \mathbf{H}$ NMR $\left(500 \mathrm{MHz}, \mathrm{CD}_{3} \mathrm{CN}\right) \delta 6.10(\mathrm{dt}, J=$ $17.9,4.9 \mathrm{~Hz}, 1 \mathrm{H}), 5.68(\mathrm{dt}, J=17.9,1.7 \mathrm{~Hz}, 1 \mathrm{H}), 4.59$ (dd, $J=4.9,1.7 \mathrm{~Hz}, 2 \mathrm{H}), 3.96(\mathrm{~d}, J=17.0$ $\mathrm{Hz}, 2 \mathrm{H}), 3.79$ (d, $J=16.9 \mathrm{~Hz}, 2 \mathrm{H}), 2.76$ (s, 3H), 2.03 (s, 3H). ${ }^{13} \mathbf{C}$ NMR $\left(126 \mathrm{MHz}, \mathrm{CD}_{3} \mathrm{CN}\right) \delta$ 171.3, 169.2, 139.8, 66.3, 62.3, 47.6, 21.0. ${ }^{11} \mathbf{B}$ NMR $\left(128 \mathrm{MHz}, \mathrm{CD}_{3} \mathrm{CN}\right) \delta 3.3$. FTIR (neat): $v=$ 2964, 1748, 1725, 1650, 1453, 1434, 1382, 1340, 1307, 1294, 1268, 1232, 1196, 1151, 1112, 1085, 1072, 1023, 1003, 955, 895, 861, 630, 603. HRMS (ESI-TOF) m/z: $\left[\mathrm{M}+\mathrm{NH}_{4}\right]^{+}$Calcd for $\mathrm{C}_{10-}$ $\mathrm{H}_{18} \mathrm{BN}_{2} \mathrm{O}_{6} 273.1258$; Found 273.1247. Melting Point: $80-83{ }^{\circ} \mathrm{C}$.

\section{$\underline{\text { Allylic Amination }}$}

\section{MIDA (E)-(3-(dibenzylamino)prop-1-en-1-yl)boronate (5)}

A dry 2-dram vial backfilled with nitrogen and equipped with Teflon-
coated stir bar and septum was charged with $2 \mathbf{2 a}(84 \mathrm{mg}, 0.33 \mathrm{mmol}, 1.0$

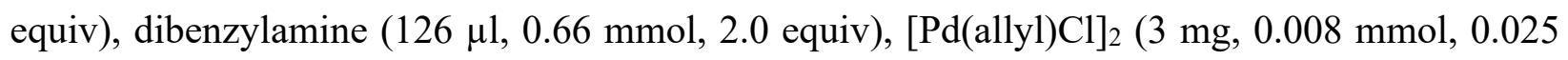
equiv), and $\mathrm{PPh}_{3}$ (4 mg, $0.017 \mathrm{mmol}, 0.05$ equiv). Then dry and degassed acetonitrile ( $3 \mathrm{ml}, 0.1$ M) was injected through the septum. The reaction was then heated to $50{ }^{\circ} \mathrm{C}$ in an oil bath for 16 hours. After 16 hours the reaction was concentrated under vacuum then purified by silica gel chromatography ( $50 \%$ acetone in hexanes with $\left.1 \% \mathrm{Et}_{3} \mathrm{~N}\right)$ to afford 5 as a beige solid $(95 \%, 0.123$ g). ${ }^{1}$ H NMR $\left(500 \mathrm{MHz}, \mathrm{CD}_{3} \mathrm{CN}\right) \delta 7.41-7.37$ (m, 4H), $7.35-7.29(\mathrm{~m}, 4 \mathrm{H}), 7.26-7.21(\mathrm{~m}, 2 \mathrm{H})$, 
$6.14(\mathrm{dt}, J=17.7,5.8 \mathrm{~Hz}, 1 \mathrm{H}), 5.69(\mathrm{dt}, J=17.7,1.6 \mathrm{~Hz}, 1 \mathrm{H}), 3.91(\mathrm{~d}, J=16.9 \mathrm{~Hz}, 2 \mathrm{H}), 3.73(\mathrm{~d}$, $J=16.9 \mathrm{~Hz}, 2 \mathrm{H}), 3.57(\mathrm{~s}, 4 \mathrm{H}), 3.73(\mathrm{~d}, J=16.9 \mathrm{~Hz}, 2 \mathrm{H}), 2.72(\mathrm{~s}, 3 \mathrm{H}) .{ }^{13} \mathbf{C}$ NMR (126 MHz, $\left.\mathrm{CD}_{3} \mathrm{CN}\right) \delta 169.3,143.7,140.8,129.6,129.2,127.8,62.3,58.6,58.5,47.7 .{ }^{11} \mathbf{B}$ NMR (128 MHz, $\mathrm{CD}_{3} \mathrm{CN}$ ) $\delta$ 10.5. HRMS (DART-TOF) $\mathrm{m} / \mathrm{z}$ : $[\mathrm{M}+\mathrm{H}]^{+}$Calcd for $\mathrm{C}_{22} \mathrm{H}_{26} \mathrm{BN}_{2} \mathrm{O}_{4}$ 393.1980; Found 393.1984. MP $=154-156^{\circ} \mathrm{C}$.

\section{$\underline{\text { Arylation and Azidation }}$}

\section{MIDA (E)-(3-azidoprop-1-en-1-yl)boronate (6)}

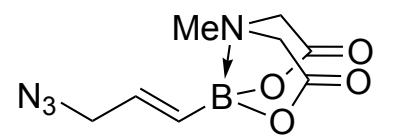

*Attention working with azides require extra precautions.*A dry flask was charged with $\mathbf{2 a}(50 \mathrm{mg}, 0.2 \mathrm{mmol}, 1.0$ equiv $),[\mathrm{Pd}(\text { allyl }) \mathrm{Cl}]_{2}(1.8 \mathrm{mg}$, 0.005 mmol, 0.025 equiv), $\mathrm{PPh}_{3}\left(2.6 \mathrm{mg}, 0.01 \mathrm{mmol}, 0.05\right.$ equiv) and $\mathrm{NaN}_{3}$ (20 mg, 0.3 mmol, 1.5 equiv) and acetonitrile $(0.08 \mathrm{M}, 2.5 \mathrm{ml})$. The reaction was then heated to $50{ }^{\circ} \mathrm{C}$ in an oil bath overnight. The reaction was then filtered through celite eluting with acetonitrile. The desired product was purified by silica gel chromatography eluting with 20-50\% acetone in hexane with $1 \% \mathrm{Et}_{3} \mathrm{~N}$ to afford 6 as a white solid $(86 \%, 0.041 \mathrm{~g}) .{ }^{1} \mathbf{H}$ NMR $\left(500 \mathrm{MHz}, \mathrm{CD}_{3} \mathrm{CN}\right) \delta 6.11(\mathrm{dt}, J=$ 17.7, $5.6 \mathrm{~Hz}, 1 \mathrm{H}), 5.76$ (dt, $J=17.7,1.5 \mathrm{~Hz}, 1 \mathrm{H}), 3.97$ (d, $J=17.0 \mathrm{~Hz}, 2 \mathrm{H}), 3.86$ (dd, $J=5.5,1.6$ $\mathrm{Hz}, 3 \mathrm{H}), 3.81$ (d, $J=16.9 \mathrm{~Hz}, 2 \mathrm{H}), 2.80$ (s, 3H). ${ }^{13} \mathbf{C}$ NMR (126 MHz, CD $\left.{ }_{3} \mathrm{CN}\right) \delta 169.2,138.7$, 62.4, 54.9, 47.8. ${ }^{11} \mathbf{B}$ NMR $\left(128 \mathrm{MHz}, \mathrm{CD}_{3} \mathrm{CN}\right) \delta$ 10.3. HRMS (DART-TOF) m/z: $\left[\mathrm{M}+\mathrm{NH}_{3}\right]^{+}$ Calcd for $\mathrm{C}_{8} \mathrm{H}_{15} \mathrm{BN}_{5} \mathrm{O}_{4} 256.1212$; Found 256.1217.

\section{MIDA (E)-(3-phenylprop-1-en-1-yl)boronate (7)}

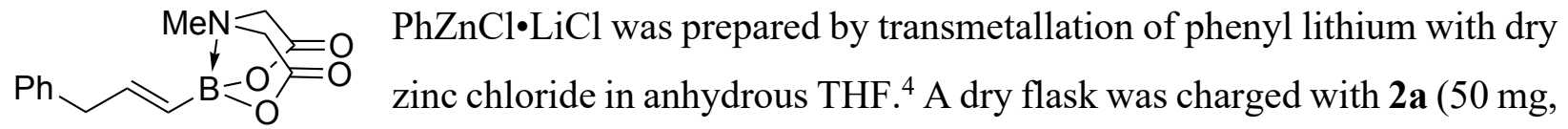
$0.2 \mathrm{mmol}, 1.0$ equiv), [Pd(allyl)Cl$]_{2}\left(1.8 \mathrm{mg}, 0.005 \mathrm{mmol}, 0.025\right.$ equiv), $\mathrm{PPh}_{3}(2.6 \mathrm{mg}, 0.01 \mathrm{mmol}$, 0.05 equiv). The flask was sealed with a rubber septum, purged and the back-filled with nitrogen. Freshly distilled acetonitrile $(0.1 \mathrm{M}, 2 \mathrm{ml})$ was added followed by the prepared solution of $\mathrm{PhZnCl} \cdot \mathrm{LiCl}\left(1.3 \mathrm{ml}, 0.40 \mathrm{mmol}, 2.0\right.$ equiv). The reaction was allowed to stir at $50{ }^{\circ} \mathrm{C}$ in an oil bath, and after 5 hours an aliquot of the reaction was analyzed by ${ }^{1} \mathrm{H}$ NMR which showed complete consumption of the starting material. The reaction was then concentrated in vacuo. Purification by silica gel column chromatography eluting with and purified by silica gel column chromatography eluting with $20-40 \% \mathrm{Et}_{2} \mathrm{O}$ in acetonitrile to afford 7 as a white solid (73\%, $40 \mathrm{mg}$ ). The spectral characteristic of this compound match what has been previously reported in the literature. ${ }^{5}$ 


\section{3-Component Coupling}

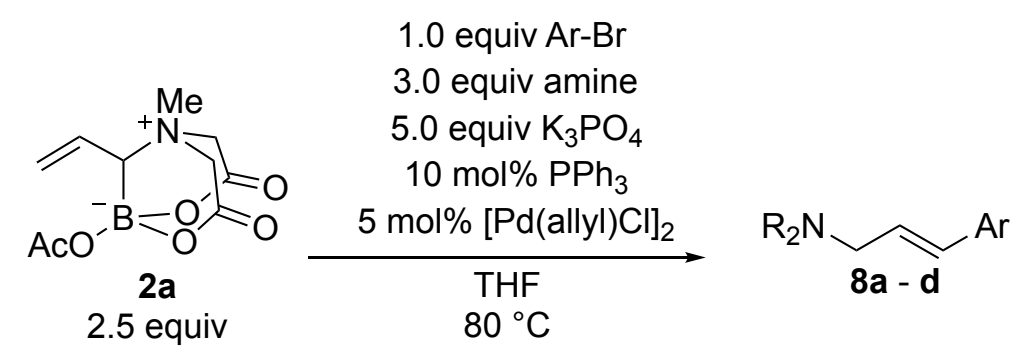

General Procedure for 3-Component Coupling (GP2) : To a $10 \mathrm{~mL}$ vial equipped with a magnetic stir bar was added $2 \mathrm{a}\left(0.128 \mathrm{~g}, 0.50 \mathrm{mmol}, 2.5\right.$ equiv), $[\mathrm{Pd}(\text { allyl }) \mathrm{Cl}]_{2}(0.004 \mathrm{~g}, 0.01 \mathrm{mmol}$, 0.05 equiv), $\mathrm{PPh}_{3}$ (0.005 g, $0.02 \mathrm{mmol}, 0.1$ equiv) and $\mathrm{K}_{3} \mathrm{PO}_{4}(0.212 \mathrm{~g}, 1.0 \mathrm{mmol}, 5.0$ equiv). If the aryl bromide is a solid, then it was added at this point $(0.2 \mathrm{mmol}, 1.0$ equiv). If the amine is a solid, then the amine was added at this point $(0.6 \mathrm{mmol}, 3.0$ equiv). The vial was sealed with a septum cap, then the system was purged of air with nitrogen using Schlenk technique 3 times. Distilled THF $(1.6 \mathrm{~mL})$ and degassed water $(0.4 \mathrm{~mL})$ were added, followed by the corresponding aryl bromide (1.0 equiv) and amine (3.0 equiv) if they are liquids. The mixture was sealed and allowed to stir at $80^{\circ} \mathrm{C}$ in an oil bath for $16 \mathrm{~h}$. Once the reaction was complete as determined by ${ }^{1} \mathrm{H}$ NMR spectroscopy, the mixture was extracted with EtOAc $(3 \times 5 \mathrm{~mL})$. The combined organic layers were loaded onto Celite and subjected to column chromatography eluted with a mixture of Acetone/Hexanes (15:85) spiked with 1\% TEA to afford the corresponding product.

\section{(E)-4-(3-(4-Methoxyphenyl)allyl)morpholine (8a)}

Compound 8a was synthesized according to GP2 and isolated in
$64 \%$ yield $(0.030 \mathrm{~g})$. The spectral characteristics of $8 \mathbf{8 a}$ match those previously reported in the literature. $^{7}$

\section{(E)- $N, N$-Diethyl-3-(o-tolyl)prop-2-en-1-amine (8b)}

Compound $\mathbf{8 b}$ was synthesized according to $\mathrm{GP} 2$ and isolated in $69 \%$ yield
$(0.028 \mathrm{~g}) .{ }^{1} \mathbf{H} \mathbf{N M R}\left(500 \mathrm{MHz}, \mathrm{CDCl}_{3}\right) \delta 7.47-7.44(\mathrm{~m}, 1 \mathrm{H}), 7.19-7.12$ (m, 3H), $6.73(\mathrm{dt}, J=15.8,1.5 \mathrm{~Hz}, 1 \mathrm{H}), 6.18(\mathrm{dt}, J=15.7,6.8 \mathrm{~Hz}, 1 \mathrm{H}), 3.30(\mathrm{dd}, J=6.8,1.5 \mathrm{~Hz}$, 2H), 2.61 (q, $J=7.2 \mathrm{~Hz}, 4 \mathrm{H}), 2.35$ (s, 3H), 1.08 (t, $J=7.2 \mathrm{~Hz}, 6 \mathrm{H}) .{ }^{13} \mathbf{C} \mathbf{N M R}\left(126 \mathrm{MHz}, \mathrm{CDCl}_{3}\right)$ 
$\delta 136.5,135.2,130.3,130.2,129.0,127.3,126.2,125.8,55.9,46.8,20.0,11.9$. HRMS (DARTTOF) m/z: [M+H] $]^{+}$Calcd for $\mathrm{C}_{14} \mathrm{H}_{22} \mathrm{~N}$ 204.1747; Found 204.1745.

\section{Cinnarizine (8c)}<smiles>C(=C/c1ccccc1)\CN1CCN(C(c2ccccc2)c2ccccc2)CC1</smiles>

8c was prepared according to GP2 and isolated in 51\% yield $(0.041 \mathrm{~g})$. After reverse phase purification, the residue was dissolved in EtOAc, 1 equiv of $\mathrm{HCl}$ (1.0 $\mathrm{M}$ in diethyl ether) was added dropwise and filtered to give product as white solid. The spectral characteristics of $\mathbf{8 c}$ match those previously reported in the literature. ${ }^{8}$

\section{Naftifine (8d)}<smiles>CN(C/C=C/c1ccccc1)Cc1cccc2ccccc12</smiles>

8d was prepared according to GP2 and isolated in $62 \%$ yield $(0.036 \mathrm{~g})$. The spectral characteristics of $\mathbf{8 d}$ match those previously reported in the literature. $^{9}$ 


\section{Stability Study of Compound 2a}

Compound 2a was dissolved in 1,4-dioxane and heated to $75^{\circ} \mathrm{C}$. Aliquots were taken every 24 hours for 3 days and analyzed by ${ }^{11} \mathrm{~B}$ NMR to determine thermal stability against protodeboronation. After 72 hours of heating no other boron signals were observed.

Thermal Stability at $75^{\circ} \mathrm{C}$

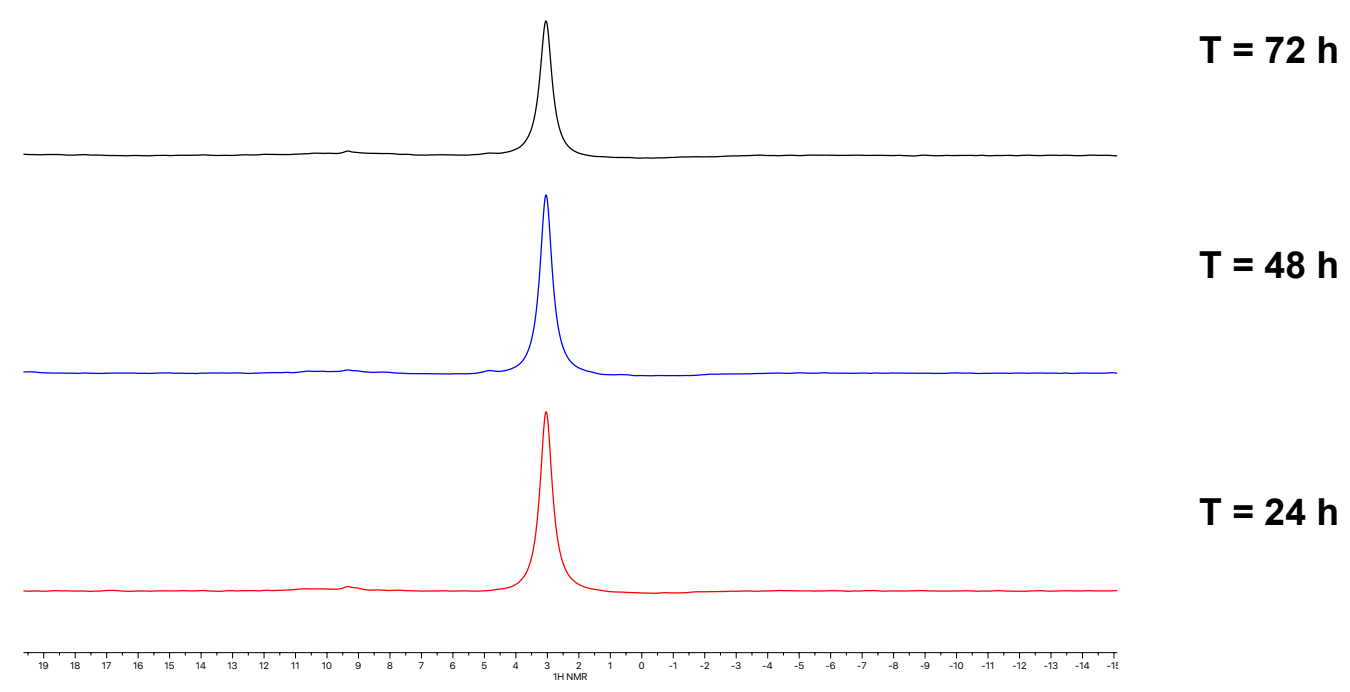

Figure S-1. ${ }^{11} \mathrm{~B}$ NMR of $\mathbf{2 a}$ in 1,4 -dioxane at $75{ }^{\circ} \mathrm{C}$ over 72 hours. 


\section{Reaction Monitoring}

The reaction was monitored by proton NMR in 1,4-dioxane using solvent suppression to remove the signal corresponding to 1,4-dioxane, with mesitylene as the internal standard. We observed the appearance of 2a immediately at the start of the reaction, and the rate of formation of $\mathbf{2 a}$ was almost identical to the rate of consumption of the starting material. The $\alpha$-acetoxyboronate (4) was not detected at any point in the course of the reaction. The linear acetate (3) started to appear only near the end of the reaction and was never present in significant quantities. This suggests that compound 2a is not formed from the rearrangement of an intermediate such as $\mathbf{3}$ or $\mathbf{4}$.

$$
\begin{aligned}
& x=\text { mesitylene standard } \\
& x=2 a \\
& x=4 \\
& x=1
\end{aligned}
$$
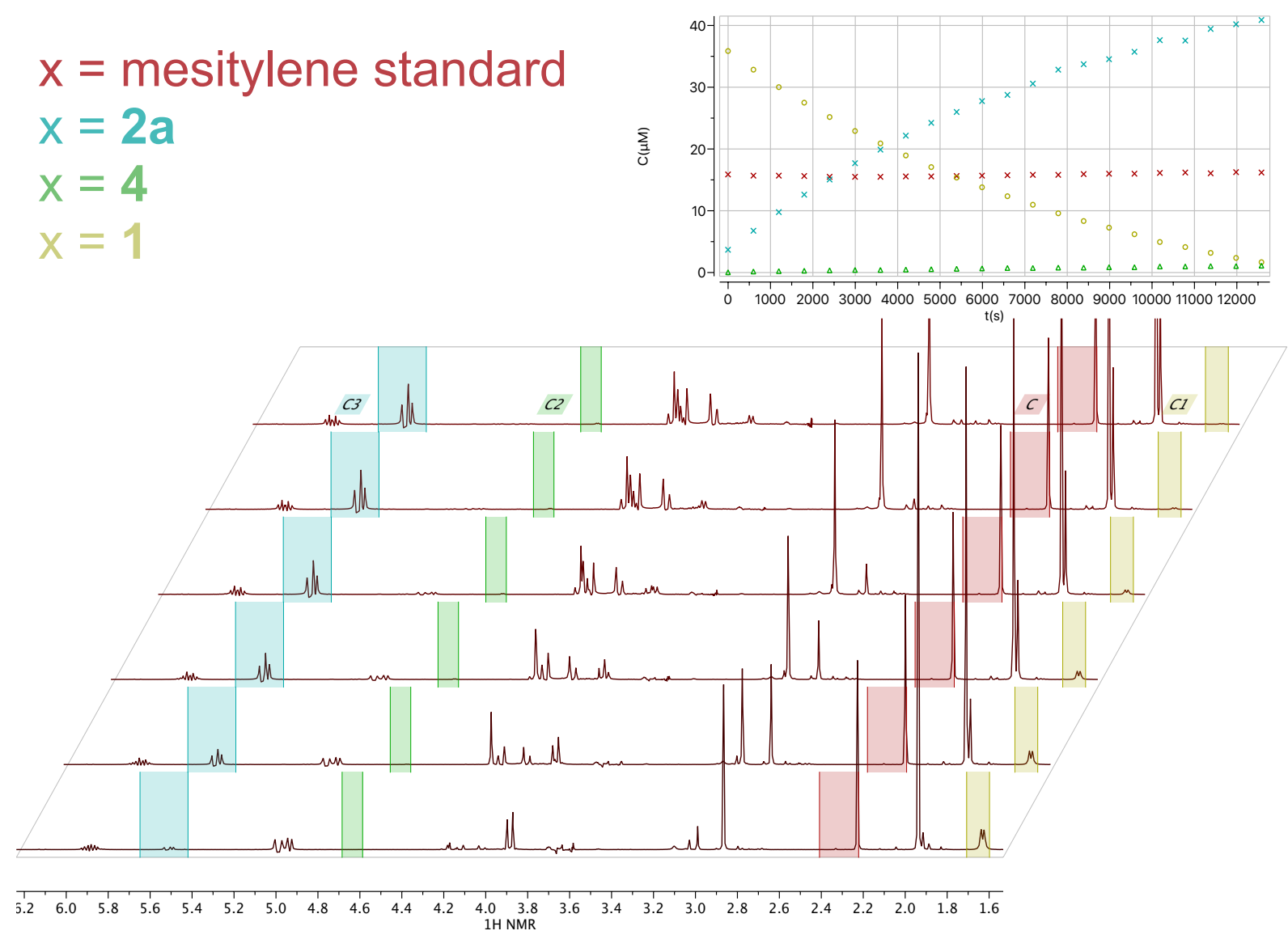

Figure S-2. ${ }^{1} \mathrm{H}$ NMR of the standard reaction conditions over 200 minutes. Mesitylene was used as internal standard and 1,4-dioxane signal was suppressed using solvent suppression. 


\section{$\underline{\text { DFT Calculations }}$}

\section{General Information: Computational Methods}

Quantum mechanical calculations were performed using Gaussian 09. ${ }^{10}$ All geometry optimizations were performed using the B3LYP functional ${ }^{11}$ and a $6-31+G(d, p)$ basis set for all non-transition metal atoms and the Lan2DZ basis set for Pd with an ultrafine grid. The optimized geometries were verified as transition state structures (one imaginary frequency) or minima (zero imaginary frequencies) by frequency calculations. Intrinsic reaction coordinate (IRC) calculations were performed to confirm that all transition state structures were linked to relevant minima. The energies of the optimized structures in the allylic oxidation study were further refined by single point calculations performed at the B3LYP/6-311+G(2d,2p) level of theory with the Lan2DZ basis for Pd applied using the integral equation formalism polarizable continuum model (IEFPCM) with the default parameters of 1,4 -dioxane $(\varepsilon=8.9)$ to account for solvent. ${ }^{12}$ The thermal corrections to the Gibbs free energies (temperature $=298.15 \mathrm{~K}$ ) computed at the lower level of theory were added to the electronic energies obtained from the single point calculations to provide the final reported Gibbs free energies. The reported activation barriers were computed with respect to the separate reagents and associated transition state. The 3D images of all optimized geometries were generated with CYLview. ${ }^{13}$ 


\section{Optimized Geometries and Table of Energies: DABN Formation}

Table S-2. Single point energies of all structures computed at the $\operatorname{IEFPCM}_{(1,4-}$ dioxane $/ \mathrm{B} 3 \mathrm{LYP} / \mathrm{Lan} 2 \mathrm{DZ}-6-311+\mathrm{G}(2 \mathrm{~d}, 2 \mathrm{p})$ level of theory when Pd is present and at the $\mathrm{IEFPCM}_{(1,4-}$ dioxane $/ \mathrm{B} 3 \mathrm{LYP} / 6-311+\mathrm{G}(2 \mathrm{~d}, 2 \mathrm{p})$ level of theory when Pd is absent. Thermal correction to Gibbs Free Energy was computed at the B3LYP/Lan2DZ-6-31+G(d,p) when Pd is present and the B3LYP/-6-31+G(d,p) level of theory when Pd is absent. For any structures with Pd present, the calculation used the Lan2DZ and $6-31+\mathrm{G}(\mathrm{d}, \mathrm{p})$ or $6-311+\mathrm{G}(2 \mathrm{~d}, 2 \mathrm{p})$ for all other atoms. All energies are reported in Hartrees.

Structure
INT-A
P1-TS1
P1-INT1
4
P1-TS2
P1-INT2
P1-TS3
P2-TS1
P2-INT1
P2-TS2
P2-INT2
2a
Acetic Acid
Acetate
1,4-Benzoquinone
Ligand Complex

Single Point Energies, E

-2536.61210630
-2536.56620289
-2536.58970329
-920.85485249
-920.83939193
-920.84558263
-920.83002197
-2536.58446943
-2536.59615851
-2536.58840007
-2536.60843855
-920.86461195
-229.17555909
-228.66393082
-381.5783236
-1615.71107982

Thermal Corrections to Gibbs Free Energies

0.420531

0.419538

0.423279

0.202605

0.200006

0.200900

0.201685

0.417024

0.417244

0.424864

0.426670

0.204479

0.034478

0.021981

0.055658

0.197218

\section{INT-A}

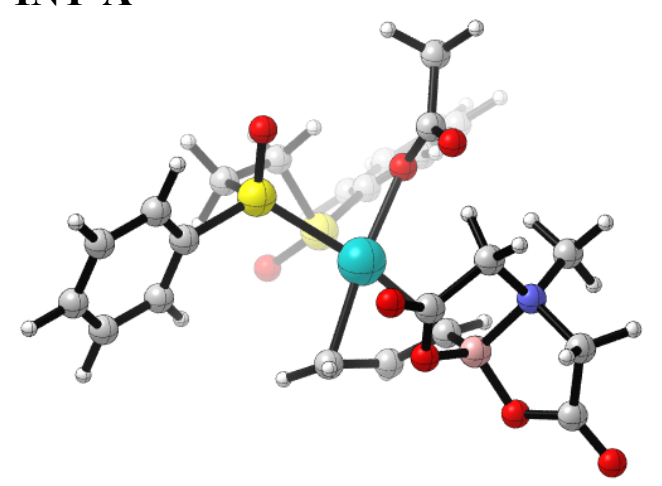

Zero-point correction $=0.497846$ (Hartree/Particle)

Thermal correction to Energy $=0.536215$

Thermal correction to Enthalpy $=0.537160$

Thermal correction to Gibbs Free Energy $=0.420531$ 
Sum of electronic and zero-point Energies $=-2535.576965$

Sum of electronic and thermal Energies $=-2535.538595$

Sum of electronic and thermal Enthalpies $=-2535.537651$

Sum of electronic and thermal Free Energies $=-2535.654280$

Number of Imaginary Frequencies $=0$

$\begin{array}{lrrr}\mathrm{O} & -3.68063700 & 0.20920100 & -0.62949700 \\ \mathrm{C} & -4.06992800 & 0.45195100 & 0.63207300 \\ \mathrm{O} & -4.48921300 & 1.51224800 & 1.03061300 \\ \mathrm{O} & -3.90203000 & -1.84424300 & -1.93229600 \\ \mathrm{C} & -4.96192700 & -2.52871800 & -1.49924400 \\ \mathrm{O} & -5.74509000 & -3.13329900 & -2.19222400 \\ \mathrm{C} & -3.87404200 & -0.79468200 & 1.48773600 \\ \mathrm{H} & -4.70607800 & -0.95877800 & 2.17496700 \\ \mathrm{C} & -5.05611300 & -2.43001400 & 0.02490800 \\ \mathrm{H} & -5.84663200 & -1.71804700 & 0.27735000 \\ \mathrm{H} & -5.29754300 & -3.39358200 & 0.47642300 \\ \mathrm{~B} & -3.15875000 & -1.14517000 & -0.86715600 \\ \mathrm{~N} & -3.72907800 & -1.91605100 & 0.50386900 \\ \mathrm{C} & -1.59196500 & -1.27024000 & -1.22434900 \\ \mathrm{H} & -1.19762300 & -2.28747400 & -1.24412900 \\ \mathrm{C} & -0.94978600 & -0.39734500 & -2.14319100 \\ \mathrm{H} & -0.15356700 & -0.77453900 & -2.78319000 \\ \mathrm{Pd} & -0.21942700 & 0.13933700 & -0.17961100 \\ \mathrm{C} & 3.42081600 & 0.57436600 & 0.47278400 \\ \mathrm{C} & 2.83164400 & 1.97254800 & 0.33622400 \\ \mathrm{C} & -2.88307100 & -3.03708500 & 1.01209400 \\ \mathrm{H} & -2.76966800 & -3.77977300 & 0.21912100 \\ \mathrm{H} & -1.91823700 & -2.63526700 & 1.31589300 \\ \mathrm{H} & -3.37079900 & -3.49601200 & 1.87633400 \\ \mathrm{H} & -2.93660700 & -0.69971200 & 2.04932100 \\ \mathrm{C} & -1.09286000 & 0.99900600 & -1.98606200 \\ \mathrm{H} & -2.01512400 & 1.42653000 & -1.60537400 \\ \mathrm{H} & -0.46258300 & 1.65422500 & -2.57907900 \\ \mathrm{H} & 3.01863900 & 0.02882600 & 1.32974400 \\ \mathrm{H} & 4.51112100 & 0.63756300 & 0.53769000 \\ \mathrm{H} & 3.28899400 & 2.66812800 & 1.04705300 \\ \mathrm{H} & 2.95972200 & 2.35855500 & -0.67911100 \\ \mathrm{H} & 3.06142000 & -0.41594600 & -1.06010600 \\ \mathrm{C} & 1.03832800 & 2.03146000 & 0.79135100 \\ \mathrm{C} & 0.62926300 & 3.68671600 & 0.15283600 \\ \mathrm{C} & 0.09911600 & 4.59496500 & 1.06851100 \\ \mathrm{C} & 0.79622900 & 4.01702900 & -1.19589300 \\ \mathrm{H} & -0.26316100 & 5.86989600 & 0.62182800 \\ \mathrm{H} & -0.01733200 & 4.29977700 & 2.10639800 \\ \mathrm{H} & & & \end{array}$




$\begin{array}{lrrr}\mathrm{H} & -0.67862700 & 6.58517800 & 1.32519800 \\ \mathrm{H} & 0.56789500 & 5.56611000 & -2.66984800 \\ \mathrm{H} & -0.38032500 & 7.20980300 & -1.06291100 \\ \mathrm{C} & 4.07264100 & -1.86315900 & -0.61574400 \\ \mathrm{C} & 5.28837600 & -2.05469200 & -1.27082000 \\ \mathrm{C} & 3.57755500 & -2.78759800 & 0.30997900 \\ \mathrm{C} & 6.04492500 & -3.19143800 & -0.96749200 \\ \mathrm{H} & 5.61765100 & -1.32463800 & -2.00477100 \\ \mathrm{C} & 4.34623000 & -3.91624200 & 0.60569100 \\ \mathrm{H} & 2.61498400 & -2.61993700 & 0.78771100 \\ \mathrm{C} & 5.57693600 & -4.11763800 & -0.03041600 \\ \mathrm{H} & 6.99538100 & -3.35482400 & -1.46740600 \\ \mathrm{H} & 3.98108000 & -4.64170700 & 1.32707000 \\ \mathrm{H} & 6.16613800 & -5.00072500 & 0.19948700 \\ \mathrm{O} & 3.77410500 & 0.32868900 & -2.18459600 \\ \mathrm{O} & 0.97453900 & 2.10258800 & 2.29585500 \\ \mathrm{O} & -1.09075100 & -1.07474600 & 2.73577100 \\ \mathrm{C} & 0.13326500 & -1.17057000 & 2.54212400 \\ \mathrm{O} & 0.72474800 & -0.97437900 & 1.40635800 \\ \mathrm{C} & 1.05865200 & -1.52484300 & 3.69914000 \\ \mathrm{H} & 1.92123600 & -2.10689800 & 3.36404600 \\ \mathrm{H} & 1.42926000 & -0.58732300 & 4.12955600 \\ \mathrm{H} & 0.51109200 & -2.06560100 & 4.47348600\end{array}$

\section{P1-TS1}

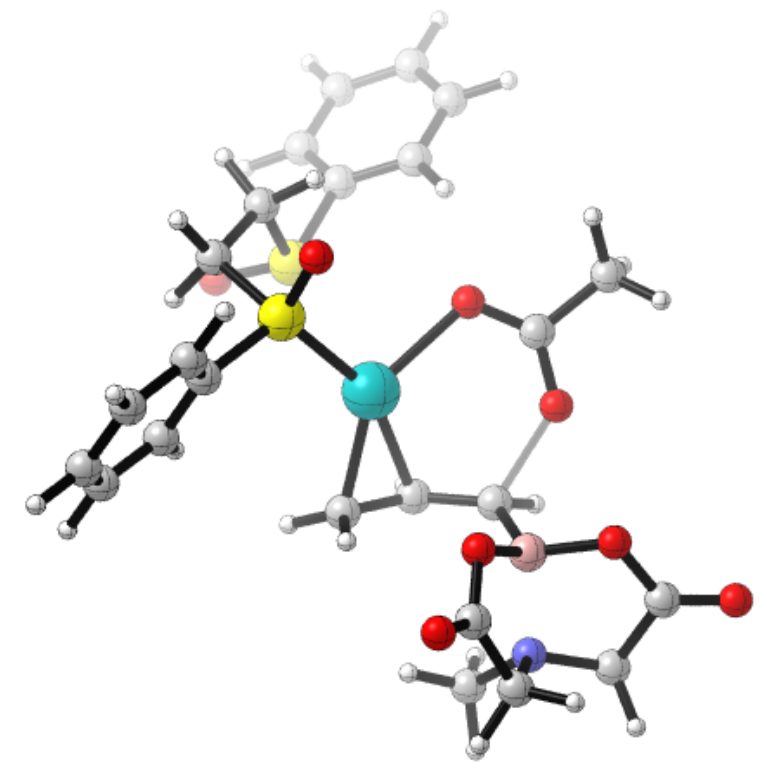

Zero-point correction $=0.496691$ (Hartree/Particle $)$

Thermal correction to Energy $=0.534400$

Thermal correction to Enthalpy $=0.535344$

Thermal correction to Gibbs Free Energy $=0.419538$

Sum of electronic and zero-point Energies $=-2535.531283$

Sum of electronic and thermal Energies $=-2535.493574$ 
Sum of electronic and thermal Enthalpies $=-2535.492629$

Sum of electronic and thermal Free Energies $=-2535.608435$

Number of Imaginary Frequencies $=0$

$\begin{array}{lrrr}\mathrm{O} & 3.93049900 & -2.18135500 & -0.89886900 \\ \mathrm{C} & 4.90061600 & -2.94594500 & -0.41114900 \\ \mathrm{O} & 5.43145100 & -3.87347800 & -0.97548000 \\ \mathrm{O} & 3.42690700 & 0.16643600 & -0.53887100 \\ \mathrm{C} & 4.59217300 & 0.78922200 & -0.35461800 \\ \mathrm{O} & 4.89257000 & 1.87244500 & -0.79319900 \\ \mathrm{C} & 5.26908600 & -2.48904400 & 1.00700400 \\ \mathrm{H} & 6.34945000 & -2.49290500 & 1.16733900 \\ \mathrm{C} & 5.52393000 & -0.06567700 & 0.51433700 \\ \mathrm{H} & 6.27833100 & -0.52101300 & -0.13281300 \\ \mathrm{H} & 6.03021600 & 0.54214600 & 1.26620400 \\ \mathrm{~B} & 3.36533300 & -1.17547000 & 0.01279900 \\ \mathrm{~N} & 4.67575100 & -1.13010300 & 1.14649500 \\ \mathrm{C} & 1.98446000 & -1.59417200 & 0.72483600 \\ \mathrm{H} & 1.97771500 & -2.63078500 & 1.05883800 \\ \mathrm{C} & 1.17181000 & -0.74185500 & 1.54894400 \\ \mathrm{H} & 0.55193500 & -1.25035500 & 2.28769900 \\ \mathrm{Pd} & -0.18335300 & 0.17429500 & 0.12979000 \\ \mathrm{C} & -3.75156900 & 0.89311000 & -0.36852300 \\ \mathrm{C} & -2.95587000 & 2.18642900 & -0.24321700 \\ \mathrm{C} & 4.35885100 & -0.78282000 & 2.55986700 \\ \mathrm{H} & 3.88852500 & 0.20020500 & 2.59368900 \\ \mathrm{H} & 3.66043700 & -1.51473700 & 2.96570900 \\ \mathrm{H} & 5.27877700 & -0.77767900 & 3.15243100 \\ \mathrm{H} & 4.80341700 & -3.16150700 & 1.73343200 \\ \mathrm{C} & 1.20022600 & 0.69470200 & 1.61272500 \\ \mathrm{H} & 1.99426000 & 1.26087900 & 1.13326900 \\ \mathrm{H} & 0.78655500 & 1.15398000 & 2.50858400 \\ \mathrm{H} & -3.54099500 & 0.36825200 & -1.30517100 \\ \mathrm{H} & -4.82365200 & 1.10291100 & -0.30260700 \\ \mathrm{H} & -3.39772800 & 2.97850800 & -0.85597500 \\ \mathrm{H} & -2.89131900 & 2.52484200 & 0.79529500 \\ \mathrm{H} & -3.39075200 & -0.24570700 & 1.05780300 \\ \mathrm{C} & -1.24422600 & 1.99083000 & -0.92402000 \\ \mathrm{C} & -0.60854100 & 3.64376500 & -0.48888800 \\ \mathrm{C} & -0.37529200 & 4.53745200 & -1.53271700 \\ \mathrm{C} & -0.34244600 & 3.98177100 & 0.84033600 \\ \mathrm{H} & 0.12416200 & 5.80944400 & -1.23295300 \\ \mathrm{H} & -57995300 & 4.22712400 & -2.55270700 \\ \mathrm{H} & & 5.25762200 & 1.12768600 \\ \mathrm{H} & 0.35051200 & 3.26081600 & 1.63654800 \\ \mathrm{H} & 6.17022700 & 0.09213600 \\ \mathrm{H} & 6.51382600 & -2.03719300 \\ \mathrm{H} & 5.53613900 & 2.15746400\end{array}$




$\begin{array}{lrrr}\mathrm{H} & 0.77003200 & 7.15827300 & 0.32012700 \\ \mathrm{C} & -4.54097100 & -1.56430600 & 0.54298400 \\ \mathrm{C} & -5.74299800 & -1.70706900 & 1.23495100 \\ \mathrm{C} & -4.16856500 & -2.44397900 & -0.47891400 \\ \mathrm{C} & -6.61101800 & -2.74270500 & 0.87361500 \\ \mathrm{H} & -5.97562100 & -1.01729400 & 2.04138600 \\ \mathrm{C} & -5.04808900 & -3.47070500 & -0.83252300 \\ \mathrm{H} & -3.20974400 & -2.32894400 & -0.97987100 \\ \mathrm{C} & -6.26647900 & -3.61975000 & -0.15944400 \\ \mathrm{H} & -7.55252300 & -2.86556500 & 1.40159500 \\ \mathrm{H} & -4.77848100 & -4.15849200 & -1.62903400 \\ \mathrm{H} & -6.94225300 & -4.42413800 & -0.43585100 \\ \mathrm{O} & -3.99732700 & 0.44593000 & 2.27329000 \\ \mathrm{O} & -1.40578500 & 1.96364600 & -2.42682100 \\ \mathrm{O} & 1.22693400 & -2.40378600 & -0.89998800 \\ \mathrm{C} & 0.04396100 & -2.36091300 & -1.39068400 \\ \mathrm{O} & -0.86698100 & -1.53102300 & -1.13525800 \\ \mathrm{C} & -0.26272900 & -3.47522200 & -2.38449900 \\ \mathrm{H} & -0.70314200 & -4.31778500 & -1.83871600 \\ \mathrm{H} & -0.98515400 & -3.12373400 & -3.12411200 \\ \mathrm{H} & 0.64934500 & -3.82242400 & -2.87209100\end{array}$

\section{P1-INT1}

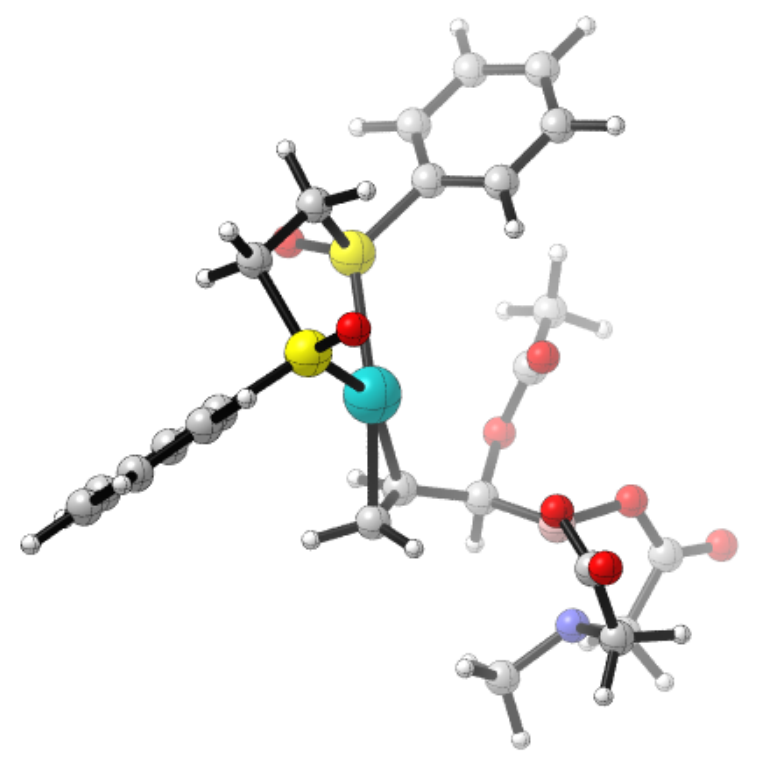

Zero-point correction $=0.498687$ (Hartree/Particle $)$

Thermal correction to Energy $=0.536488$

Thermal correction to Enthalpy $=0.537432$

Thermal correction to Gibbs Free Energy $=0.423279$

Sum of electronic and zero-point Energies $=-2535.551923$

Sum of electronic and thermal Energies $=-2535.514123$

Sum of electronic and thermal Enthalpies $=-2535.513179$ 
Sum of electronic and thermal Free Energies $=-2535.627332$

Number of Imaginary Frequencies $=0$

\begin{tabular}{|c|c|c|c|}
\hline $\mathrm{O}$ & 4.23534800 & -0.19394000 & 0.37601300 \\
\hline $\mathrm{C}$ & 5.42507600 & -0.62629800 & -0.02865600 \\
\hline $\mathrm{O}$ & 6.46507400 & -0.01341700 & 0.04020100 \\
\hline $\mathrm{O}$ & 2.28566900 & -1.40713500 & 1.21169800 \\
\hline $\mathrm{C}$ & 2.76625500 & -2.42079300 & 1.93134000 \\
\hline $\mathrm{O}$ & 2.32558700 & -2.81671600 & 2.98300500 \\
\hline $\mathrm{C}$ & 5.30477000 & -2.03865000 & -0.61506400 \\
\hline $\mathrm{H}$ & 6.12176700 & -2.68501500 & -0.28610300 \\
\hline $\mathrm{C}$ & 3.98267900 & -3.04247200 & 1.23322000 \\
\hline $\mathrm{H}$ & 4.88660000 & -2.70617000 & 1.74794100 \\
\hline $\mathrm{H}$ & 3.94660500 & -4.13272100 & 1.26878400 \\
\hline B & 3.07627500 & -1.05491700 & 0.04947500 \\
\hline $\mathrm{N}$ & 3.97181700 & -2.53235200 & -0.17650300 \\
\hline $\mathrm{C}$ & 2.32542400 & -0.58041000 & -1.31644400 \\
\hline $\mathrm{H}$ & 2.90738200 & -1.01774300 & -2.14438100 \\
\hline $\mathrm{C}$ & 0.88262400 & -0.95004200 & -1.62313900 \\
\hline $\mathrm{H}$ & 0.59302700 & -0.66076400 & -2.63414900 \\
\hline $\mathrm{Pd}$ & -0.79692300 & -0.21287500 & -0.37461700 \\
\hline $\mathrm{C}$ & -3.20771900 & 2.05375200 & 0.44916900 \\
\hline $\mathrm{C}$ & -3.93919100 & 0.73997400 & 0.23020000 \\
\hline $\mathrm{C}$ & 3.40292300 & -3.55422800 & -1.09749700 \\
\hline $\mathrm{H}$ & 2.41618300 & -3.85173000 & -0.74197200 \\
\hline $\mathrm{H}$ & 3.30235400 & -3.13122300 & -2.09713700 \\
\hline $\mathrm{H}$ & 4.06100800 & -4.42780500 & -1.13366600 \\
\hline $\mathrm{H}$ & 5.32603300 & -1.97437100 & -1.70685300 \\
\hline $\mathrm{C}$ & 0.17626000 & -2.01068100 & -1.03520400 \\
\hline $\mathrm{H}$ & 0.55739000 & -2.53526600 & -0.16436400 \\
\hline $\mathrm{H}$ & -0.57686800 & -2.54560100 & -1.6100210 \\
\hline $\mathrm{H}$ & -2.90447900 & 2.17653000 & 1.49298600 \\
\hline $\mathrm{H}$ & -3.81599200 & 2.90815800 & 0.13726000 \\
\hline $\mathrm{H}$ & -4.85593500 & 0.68832000 & 0.82565600 \\
\hline $\mathrm{H}$ & -4.16919500 & 0.57137600 & -0.82560000 \\
\hline S & -1.68172200 & 2.12979800 & -0.61622200 \\
\hline $\mathrm{S}$ & -2.86736800 & -0.65249200 & 0.83668800 \\
\hline $\mathrm{C}$ & -3.87207500 & -2.03636100 & 0.20458200 \\
\hline $\mathrm{C}$ & -4.58364900 & -2.79935600 & 1.12911000 \\
\hline $\mathrm{C}$ & -3.87895900 & -2.34097000 & -1.1593390 \\
\hline $\mathrm{C}$ & -5.34038800 & -3.88283300 & 0.67008400 \\
\hline $\mathrm{H}$ & -4.53066400 & -2.54061600 & 2.1825010 \\
\hline $\mathrm{C}$ & -4.64403600 & -3.42041600 & -1.6064010 \\
\hline $\mathrm{H}$ & -3.29150100 & -1.75041500 & -1.8580780 \\
\hline $\mathrm{C}$ & -5.37388700 & -4.19069200 & -0.6928770 \\
\hline $\mathrm{H}$ & -5.89969500 & -4.48613600 & 1.3793580 \\
\hline $\mathrm{H}$ & -4.66443800 & -3.66377200 & -2.6648120 \\
\hline $\mathrm{H}$ & -5.96194300 & -5.03347200 & -1.0446530 \\
\hline
\end{tabular}




$\begin{array}{lrrr}\mathrm{C} & -0.99149000 & 3.64469000 & 0.13330000 \\ \mathrm{C} & -1.13502300 & 4.84168500 & -0.56817000 \\ \mathrm{C} & -0.32277700 & 3.58190200 & 1.35800200 \\ \mathrm{C} & -0.61684200 & 6.01537900 & -0.01119300 \\ \mathrm{H} & -1.63901600 & 4.83673600 & -1.53023500 \\ \mathrm{C} & 0.18294100 & 4.76280000 & 1.90671900 \\ \mathrm{H} & -0.16808600 & 2.63180900 & 1.86014600 \\ \mathrm{C} & 0.03523900 & 5.97665000 & 1.22537200 \\ \mathrm{H} & -0.72143800 & 6.95608000 & -0.54431800 \\ \mathrm{H} & 0.70727600 & 4.72901300 & 2.85704200 \\ \mathrm{H} & 0.43792200 & 6.88970000 & 1.65460200 \\ \mathrm{O} & -2.17286600 & 2.49846500 & -2.00501500 \\ \mathrm{O} & -3.00536000 & -0.65207700 & 2.34581800 \\ \mathrm{O} & 2.56829400 & 0.83879500 & -1.61250500 \\ \mathrm{C} & 2.30180900 & 1.75271200 & -0.65572100 \\ \mathrm{O} & 1.82784700 & 1.47319300 & 0.42726100 \\ \mathrm{C} & 2.70344500 & 3.13747800 & -1.10165500 \\ \mathrm{H} & 2.45449300 & 3.30026300 & -2.15298800 \\ \mathrm{H} & 2.21448800 & 3.88085700 & -0.47207100 \\ \mathrm{H} & 3.78932700 & 3.23613600 & -0.99201800\end{array}$

4

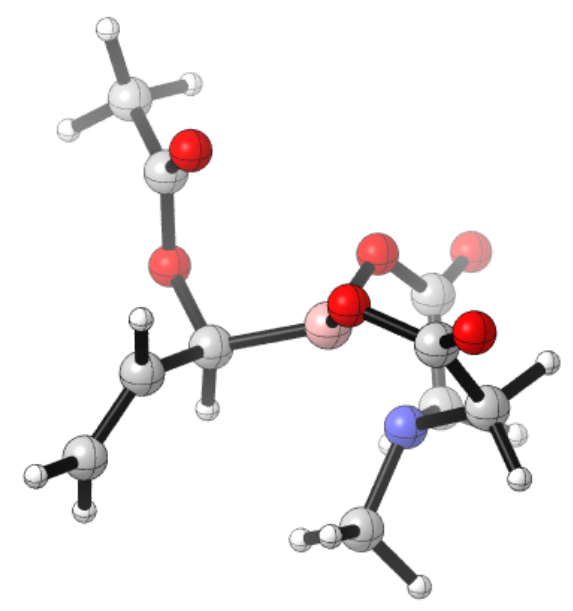

Zero-point correction $=0.248995$ (Hartree/Particle)

Thermal correction to Energy $=0.266740$

Thermal correction to Enthalpy $=0.267685$

Thermal correction to Gibbs Free Energy $=0.202605$

Sum of electronic and zero-point Energies $=-920.347665$

Sum of electronic and thermal Energies $=-920.329920$

Sum of electronic and thermal Enthalpies $=-920.328976$

Sum of electronic and thermal Free Energies $=-920.394055$

Number of Imaginary Frequencies $=0$ 


$\begin{array}{lrrr}\mathrm{O} & 0.03726000 & 1.39118300 & 0.40714700 \\ \mathrm{C} & 0.70300300 & 2.23734000 & -0.37516200 \\ \mathrm{O} & 0.59445200 & 3.44018900 & -0.37623200 \\ \mathrm{O} & 0.65613500 & -0.81815600 & 1.26930100 \\ \mathrm{C} & 1.96571700 & -0.78470600 & 1.51460700 \\ \mathrm{O} & 2.52590300 & -1.28610100 & 2.45803300 \\ \mathrm{C} & 1.64665000 & 1.46463000 & -1.30834500 \\ \mathrm{H} & 2.62457700 & 1.94469100 & -1.38466900 \\ \mathrm{C} & 2.68802300 & -0.00280700 & 0.41066200 \\ \mathrm{H} & 2.92075300 & 0.99633600 & 0.78814400 \\ \mathrm{H} & 3.61776400 & -0.49174900 & 0.11546600 \\ \mathrm{~B} & 0.23430800 & -0.04244600 & 0.11582200 \\ \mathrm{~N} & 1.72341800 & 0.09315300 & -0.73502700 \\ \mathrm{C} & -0.96511500 & -0.69016700 & -0.77972000 \\ \mathrm{H} & -0.74422900 & -0.56163400 & -1.84613700 \\ \mathrm{C} & -1.21440100 & -2.15501600 & -0.53476200 \\ \mathrm{H} & -1.38410600 & -2.44393700 & 0.49842500 \\ \mathrm{C} & 2.01812100 & -0.95855700 & -1.74712600 \\ \mathrm{H} & 1.96505800 & -1.93870500 & -1.27038600 \\ \mathrm{H} & 1.27924500 & -0.91443400 & -2.54649800 \\ \mathrm{H} & 3.01679500 & -0.80256600 & -2.16496400 \\ \mathrm{H} & 1.19980500 & 1.41583900 & -2.30552000 \\ \mathrm{C} & -1.21157600 & -3.07601600 & -1.50419000 \\ \mathrm{H} & -1.05658700 & -2.81173500 & -2.54923700 \\ \mathrm{H} & -1.37334000 & -4.12824100 & -1.28839900 \\ \mathrm{O} & -2.20366600 & 0.10083800 & -0.67936200 \\ \mathrm{C} & -2.77201600 & 0.24928000 & 0.54012100 \\ \mathrm{O} & -2.35130500 & -0.28623100 & 1.54414100 \\ \mathrm{C} & -3.96426500 & 1.17131300 & 0.47587300 \\ \mathrm{H} & -3.60718400 & 2.20507300 & 0.41403400 \\ \mathrm{H} & -4.56939000 & 0.97145000 & -0.41166600 \\ \mathrm{H} & -4.55972100 & 1.05423900 & 1.38151900\end{array}$

\section{P1-TS2}




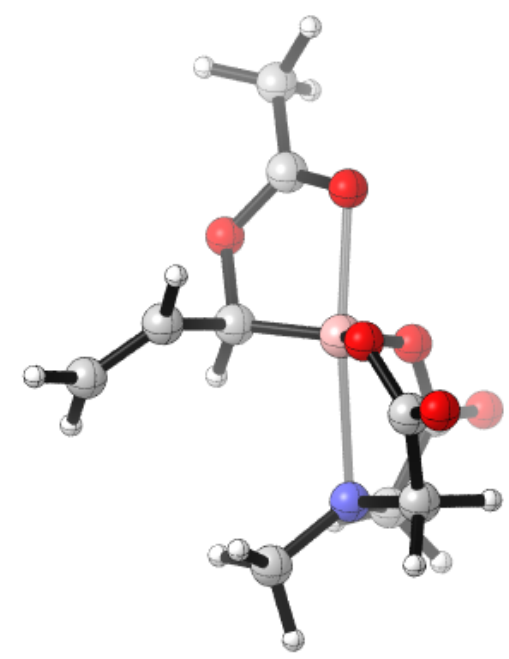

Zero-point correction $=0.246786$ (Hartree/Particle)

Thermal correction to Energy $=0.264580$

Thermal correction to Enthalpy $=0.265524$

Thermal correction to Gibbs Free Energy $=0.200006$

Sum of electronic and zero-point Energies $=-920.337607$

Sum of electronic and thermal Energies $=-920.319813$

Sum of electronic and thermal Enthalpies = -920.318869

Sum of electronic and thermal Free Energies $=\mathbf{- 9 2 0 . 3 8 4 3 8 7}$

Number of Imaginary Frequencies $=0$

$\begin{array}{lrrr}\mathrm{O} & 0.00553900 & 1.49875700 & 0.21223700 \\ \mathrm{C} & 0.84365900 & 2.14882400 & -0.61022700 \\ \mathrm{O} & 0.78185400 & 3.34134600 & -0.79301300 \\ \mathrm{O} & 0.37582100 & -0.60163800 & 1.43278500 \\ \mathrm{C} & 1.68449700 & -0.63274100 & 1.73370000 \\ \mathrm{O} & 2.10575300 & -1.08749800 & 2.76973000 \\ \mathrm{C} & 1.88892300 & 1.26440200 & -1.29576800 \\ \mathrm{H} & 2.84368200 & 1.80945700 & -1.30293100 \\ \mathrm{C} & 2.60055000 & -0.05279800 & 0.65021000 \\ \mathrm{H} & 2.85592300 & 0.96648400 & 0.96313000 \\ \mathrm{H} & 3.53261800 & -0.62741900 & 0.65213100 \\ \mathrm{~B} & -0.19503500 & 0.09476400 & 0.34776700 \\ \mathrm{~N} & 1.94918200 & -0.04442100 & -0.66277700 \\ \mathrm{C} & -1.05251000 & -0.69681800 & -0.77285700 \\ \mathrm{H} & -0.70217900 & -0.46439600 & -1.78228500 \\ \mathrm{C} & -1.11315700 & -2.18501400 & -0.58155100 \\ \mathrm{H} & -1.39190200 & -2.52663800 & 0.41389000 \\ \mathrm{C} & 2.40812200 & -1.12784200 & -1.53741700 \\ \mathrm{H} & 2.28929600 & -2.08507800 & -1.02432500 \\ \mathrm{H} & 1.79714200 & -1.15345100 & -2.44337900 \\ \mathrm{H} & 3.46386100 & -1.01170500 & -1.82947200 \\ \mathrm{H} & 1.57920000 & 1.14762300 & -2.34024700 \\ \mathrm{C} & -0.83527700 & -3.07561400 & -1.53750600 \\ \mathrm{H} & -0.55490600 & -2.76772700 & -2.54270500\end{array}$




$\begin{array}{llcc}\mathrm{H} & -0.87753100 & -4.14411600 & -1.34817100 \\ \mathrm{O} & -2.44861900 & -0.18097600 & -0.78806100 \\ \mathrm{C} & -2.83076500 & 0.33353600 & 0.38063300 \\ \mathrm{O} & -2.04515400 & 0.41187400 & 1.32044500 \\ \mathrm{C} & -4.24961700 & 0.81891500 & 0.40813500 \\ \mathrm{H} & -4.27962700 & 1.82657200 & -0.02118000 \\ \mathrm{H} & -4.89522900 & 0.17404700 & -0.19102700 \\ \mathrm{H} & -4.59756200 & 0.86944500 & 1.44008400\end{array}$

\section{P1-INT2}

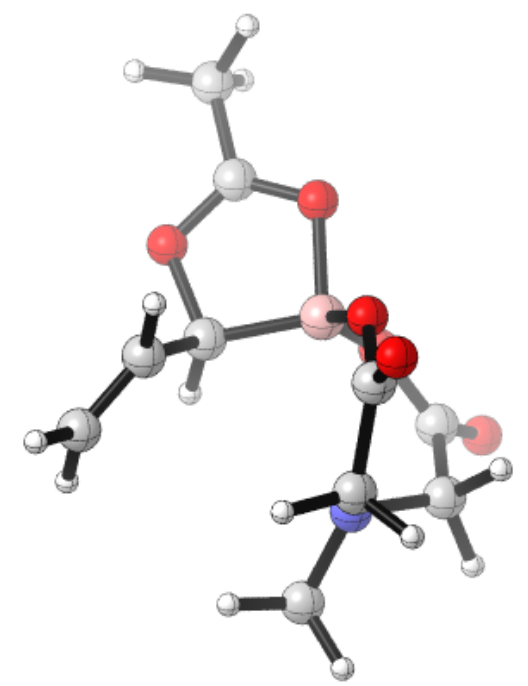

Zero-point correction $=0.247755$ (Hartree/Particle)

Thermal correction to Energy $=0.265723$

Thermal correction to Enthalpy $=0.266667$

Thermal correction to Gibbs Free Energy $=0.200900$

Sum of electronic and zero-point Energies $=-920.342870$

Sum of electronic and thermal Energies $=-920.324902$

Sum of electronic and thermal Enthalpies $=-920.323958$

Sum of electronic and thermal Free Energies $=-\mathbf{9 2 0 . 3 8 9 7 2 5}$

Number of Imaginary Frequencies $=0$

$\begin{array}{lrrr}\mathrm{O} & -0.09577000 & 1.67643700 & -0.13270800 \\ \mathrm{C} & 1.12380400 & 2.20485200 & -0.27468600 \\ \mathrm{O} & 1.27859600 & 3.36706800 & -0.58571300 \\ \mathrm{O} & 0.09679600 & -0.21204100 & 1.47760800 \\ \mathrm{C} & 1.15963000 & -1.00022600 & 1.68226400 \\ \mathrm{O} & 1.29589900 & -1.61225800 & 2.71989500 \\ \mathrm{C} & 2.30732500 & 1.27270300 & -0.01084400 \\ \mathrm{H} & 2.49665800 & 1.29653300 & 1.06990600 \\ \mathrm{C} & 2.21541400 & -1.09652300 & 0.57877500 \\ \mathrm{H} & 3.19874500 & -1.07070100 & 1.07855000 \\ \mathrm{H} & 2.11163600 & -2.09256400 & 0.13595400 \\ \mathrm{~B} & -0.52248700 & 0.36831600 & 0.31416500 \\ \mathrm{~N} & 2.05541100 & -0.09710300 & -0.46028400\end{array}$




$\begin{array}{lrrr}\mathrm{C} & -1.03199700 & -0.61705100 & -0.90359800 \\ \mathrm{H} & -0.69926300 & -0.27555100 & -1.88478500 \\ \mathrm{C} & -0.87138300 & -2.09552400 & -0.76010000 \\ \mathrm{H} & -1.17157900 & -2.52414900 & 0.19583400 \\ \mathrm{C} & 2.74072600 & -0.42428600 & -1.70842400 \\ \mathrm{H} & 2.40892200 & -1.40406500 & -2.06387000 \\ \mathrm{H} & 2.48850200 & 0.31785300 & -2.47183700 \\ \mathrm{H} & 3.84005000 & -0.44669200 & -1.60350900 \\ \mathrm{H} & 3.18626600 & 1.71503000 & -0.49264200 \\ \mathrm{C} & -0.37373400 & -2.88920300 & -1.71348900 \\ \mathrm{H} & -0.06997800 & -2.49569900 & -2.68087700 \\ \mathrm{H} & -0.24888800 & -3.95642800 & -1.55588400 \\ \mathrm{O} & -2.53758500 & -0.36224600 & -0.93366800 \\ \mathrm{C} & -2.88354000 & 0.35670800 & 0.09265800 \\ \mathrm{O} & -1.99966800 & 0.75012600 & 0.89407900 \\ \mathrm{C} & -4.32208600 & 0.70305900 & 0.26461300 \\ \mathrm{H} & -4.46973200 & 1.73477000 & -0.07341100 \\ \mathrm{H} & -4.95388500 & 0.03868000 & -0.32476600 \\ \mathrm{H} & -4.58398700 & 0.65737800 & 1.32354000\end{array}$

\section{P1-TS3}

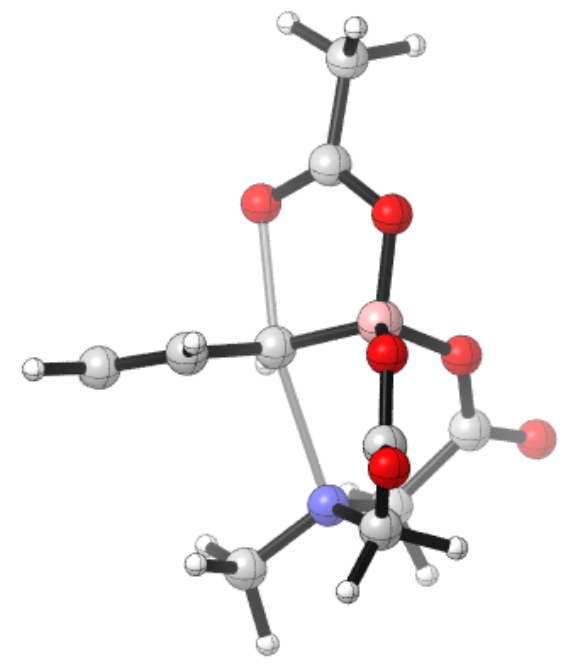

Zero-point correction $=0.247030($ Hartree/Particle $)$

Thermal correction to Energy $=0.264412$

Thermal correction to Enthalpy $=0.265356$

Thermal correction to Gibbs Free Energy $=0.201685$

Sum of electronic and zero-point Energies $=-920.327144$

Sum of electronic and thermal Energies $=-920.309762$

Sum of electronic and thermal Enthalpies $=-920.308818$

Sum of electronic and thermal Free Energies $=-920.372489$

Number of Imaginary Frequencies $=0$

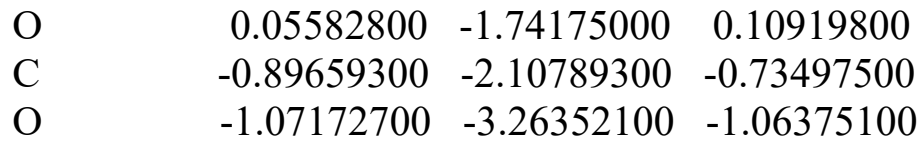




$\begin{array}{lrrr}\mathrm{O} & -0.04985500 & 0.10034800 & 1.72826900 \\ \mathrm{C} & -1.32632900 & 0.39515500 & 1.92319900 \\ \mathrm{O} & -1.74037900 & 0.84232600 & 2.97413500 \\ \mathrm{C} & -1.80760100 & -1.02473900 & -1.32541600 \\ \mathrm{H} & -2.81841600 & -1.45233800 & -1.38486800 \\ \mathrm{C} & -2.32269500 & 0.11413100 & 0.78765900 \\ \mathrm{H} & -2.70348600 & -0.90231900 & 0.94860300 \\ \mathrm{H} & -3.16318900 & 0.79354400 & 0.94220100 \\ \mathrm{~B} & 0.55286900 & -0.40547200 & 0.48784200 \\ \mathrm{~N} & -1.80087600 & 0.24900800 & -0.59460500 \\ \mathrm{C} & 0.47665300 & 0.70052600 & -0.69397200 \\ \mathrm{H} & 0.38833800 & 0.41650400 & -1.73771800 \\ \mathrm{C} & 0.45164800 & 2.13751700 & -0.42833000 \\ \mathrm{H} & 0.44933900 & 2.44207000 & 0.61609000 \\ \mathrm{C} & -2.50437100 & 1.29924400 & -1.34229400 \\ \mathrm{H} & -2.43687800 & 2.24501900 & -0.80175100 \\ \mathrm{H} & -2.03730500 & 1.43054000 & -2.32195000 \\ \mathrm{H} & -3.56468700 & 1.04566700 & -1.48981200 \\ \mathrm{H} & -1.47771900 & -0.84588200 & -2.35483400 \\ \mathrm{C} & 0.47304300 & 3.06981000 & -1.39477000 \\ \mathrm{H} & 0.50503900 & 2.79913700 & -2.44747500 \\ \mathrm{H} & 0.48768900 & 4.12996300 & -1.15956600 \\ \mathrm{O} & 2.54613800 & 0.51721800 & -0.97278400 \\ \mathrm{C} & 2.87591300 & -0.16908800 & 0.01558100 \\ \mathrm{O} & 1.99512700 & -0.63540400 & 0.85095600 \\ \mathrm{C} & 4.30906400 & -0.50743200 & 0.31277100 \\ \mathrm{H} & 4.54260500 & -0.23225300 & 1.34511000 \\ \mathrm{H} & 4.44358600 & -1.59014200 & 0.22427400 \\ \mathrm{H} & 4.97342900 & 0.00768100 & -0.38017200\end{array}$

2a

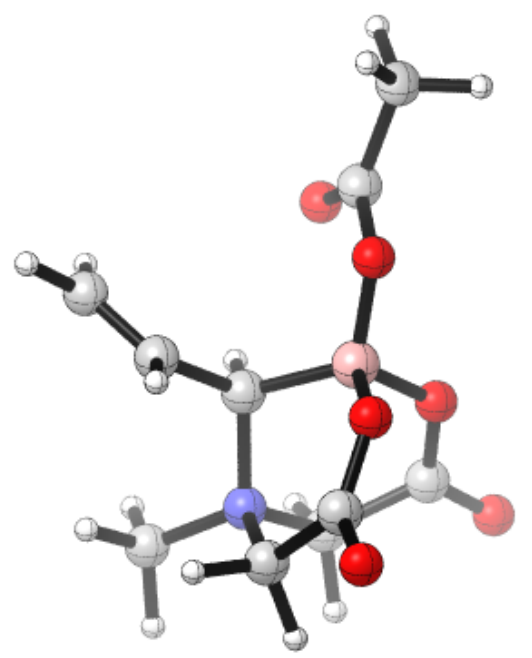

Zero-point correction $=0.249920$ (Hartree/Particle $)$

Thermal correction to Energy $=0.267325$

Thermal correction to Enthalpy $=0.268269$ 
Thermal correction to Gibbs Free Energy $=0.204479$

Sum of electronic and zero-point Energies $=-920.355758$

Sum of electronic and thermal Energies $=-\mathbf{9 2 0 . 3 3 8 3 5 3}$

Sum of electronic and thermal Enthalpies $=-920.337408$

Sum of electronic and thermal Free Energies $=-920.401198$

Number of Imaginary Frequencies $=0$

$\begin{array}{lrrr}\mathrm{O} & 0.19963400 & -1.71243300 & -0.06752500 \\ \mathrm{C} & -0.80999200 & -1.98852900 & -0.85361900 \\ \mathrm{O} & -1.11602600 & -3.10177500 & -1.22984300 \\ \mathrm{O} & -0.25871800 & -0.25104800 & 1.78836800 \\ \mathrm{C} & -1.53987500 & 0.01190700 & 1.84710500 \\ \mathrm{O} & -2.21725700 & -0.04955900 & 2.85307900 \\ \mathrm{C} & -1.64006200 & -0.82447000 & -1.42559400 \\ \mathrm{H} & -2.68964000 & -1.12091600 & -1.44170900 \\ \mathrm{C} & -2.27157900 & 0.53035500 & 0.58751300 \\ \mathrm{H} & -3.19663700 & -0.03543600 & 0.46719700 \\ \mathrm{H} & -2.53805000 & 1.56613300 & 0.80459000 \\ \mathrm{~B} & 0.48885000 & -0.35729700 & 0.49693100 \\ \mathrm{~N} & -1.53352300 & 0.52548400 & -0.74204500 \\ \mathrm{C} & -0.02431300 & 0.82018200 & -0.52325100 \\ \mathrm{H} & 0.43410800 & 0.69716800 & -1.50679600 \\ \mathrm{C} & 0.21090800 & 2.20962400 & -0.01423600 \\ \mathrm{H} & -0.16656600 & 2.45140500 & 0.97901000 \\ \mathrm{C} & -2.17999900 & 1.53889400 & -1.64483700 \\ \mathrm{H} & -2.10005400 & 2.52411000 & -1.18827400 \\ \mathrm{H} & -1.65418900 & 1.54068100 & -2.60031400 \\ \mathrm{H} & -3.22781100 & 1.26760100 & -1.79079300 \\ \mathrm{H} & -1.30477400 & -0.68298600 & -2.45614200 \\ \mathrm{C} & 0.96159000 & 3.11269900 & -0.65432700 \\ \mathrm{H} & 1.40637000 & 2.90164200 & -1.62367100 \\ \mathrm{H} & 1.18855300 & 4.07547400 & -0.20629900 \\ \mathrm{O} & 2.69841900 & -0.11761500 & -1.24220500 \\ \mathrm{C} & 2.87543000 & -0.25686900 & -0.04153800 \\ \mathrm{O} & 1.88853900 & -0.29560200 & 0.86324100 \\ \mathrm{C} & 4.23443600 & -0.40404600 & 0.59812500 \\ \mathrm{H} & 4.32455900 & -1.40901300 & 1.02367800 \\ \mathrm{H} & 5.01531600 & -0.24876500 & -0.14621800 \\ \mathrm{H} & 4.34323300 & 0.30677300 & 1.42243100\end{array}$

\section{P2-TS1}




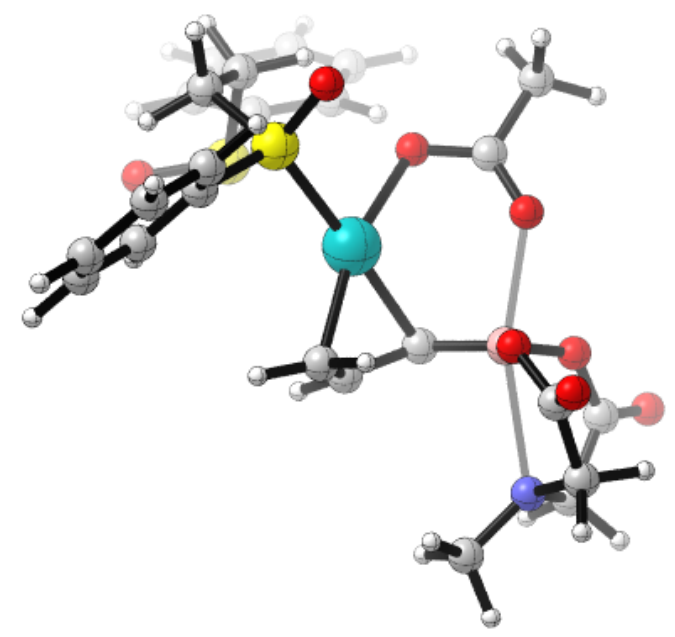

Zero-point correction $=0.494972$ (Hartree/Particle)

Thermal correction to Energy $=0.533544$

Thermal correction to Enthalpy $=0.534488$

Thermal correction to Gibbs Free Energy $=0.417024$

Sum of electronic and zero-point Energies $=-2535.551848$

Sum of electronic and thermal Energies $=-2535.513276$

Sum of electronic and thermal Enthalpies $=-2535.512332$

Sum of electronic and thermal Free Energies $=-2535.629796$

Number of Imaginary Frequencies $=0$

$\begin{array}{lrrr}\mathrm{O} & -3.43276200 & -2.18231400 & 0.42938300 \\ \mathrm{C} & -4.25063900 & -2.91859700 & -0.33516600 \\ \mathrm{O} & -4.45565800 & -4.09190300 & -0.12642200 \\ \mathrm{O} & -3.33416200 & 0.24445500 & 0.75131900 \\ \mathrm{C} & -4.62395600 & 0.62834800 & 0.74649600 \\ \mathrm{O} & -5.03062900 & 1.53059600 & 1.43981600 \\ \mathrm{C} & -4.93689100 & -2.16458900 & -1.47983400 \\ \mathrm{H} & -5.98311200 & -2.50464100 & -1.52068900 \\ \mathrm{C} & -5.52988400 & -0.13411300 & -0.23151500 \\ \mathrm{H} & -6.04384900 & -0.90825800 & 0.35065200 \\ \mathrm{H} & -6.30077000 & 0.56348200 & -0.57797500 \\ \mathrm{~B} & -2.82149200 & -0.93138100 & 0.16982600 \\ \mathrm{~N} & -4.77594500 & -0.73016100 & -1.33187900 \\ \mathrm{C} & -1.65709600 & -0.87228100 & -0.89947900 \\ \mathrm{H} & -1.29923900 & -1.85300400 & -1.21667300 \\ \mathrm{C} & -1.29511000 & 0.18608800 & -1.77821300 \\ \mathrm{H} & -0.73431000 & -0.05060200 & -2.68159800 \\ \mathrm{Pd} & -0.07612000 & 0.34435000 & 0.01937100 \\ \mathrm{C} & 3.49072600 & 0.05183400 & 0.70317700 \\ \mathrm{C} & 3.20802800 & 1.54701400 & 0.67244300 \\ \mathrm{C} & -4.83084400 & 0.03860000 & -2.57245100 \\ \mathrm{H} & -4.55226800 & 1.07736400 & -2.37252100 \\ \mathrm{H} & -4.11288900 & -0.36902700 & -3.29001500 \\ \mathrm{H} & -5.83181000 & 0.03235600 & -3.03539700 \\ \mathrm{H} & -4.45982000 & -2.48687100 & -2.41222500\end{array}$




$\begin{array}{lrrr}\mathrm{C} & -1.31715500 & 1.52593200 & -1.33639200 \\ \mathrm{H} & -2.05156600 & 1.86472000 & -0.61045400 \\ \mathrm{H} & -0.85787400 & 2.29312700 & -1.95218900 \\ \mathrm{H} & 2.93893000 & -0.47537200 & 1.48542800 \\ \mathrm{H} & 4.56646700 & -0.11331800 & 0.81430300 \\ \mathrm{H} & 3.72917800 & 2.06985100 & 1.48093300 \\ \mathrm{H} & 3.50301800 & 1.97881400 & -0.28796900 \\ \mathrm{~S} & 3.01979300 & -0.70226900 & -0.93436100 \\ \mathrm{~S} & 1.43224700 & 1.96908900 & 1.00352700 \\ \mathrm{C} & 1.38396100 & 3.61890200 & 0.23346700 \\ \mathrm{C} & 1.06408300 & 4.69413500 & 1.06021400 \\ \mathrm{C} & 1.61939300 & 3.78543500 & -1.13512400 \\ \mathrm{C} & 0.98978300 & 5.97488800 & 0.50128100 \\ \mathrm{H} & 0.88442400 & 4.51970700 & 2.11653900 \\ \mathrm{C} & 1.54733800 & 5.06955500 & -1.67797700 \\ \mathrm{H} & 1.86175900 & 2.93784900 & -1.77121700 \\ \mathrm{C} & 1.23095200 & 6.16254800 & -0.86164000 \\ \mathrm{H} & 0.74245700 & 6.82188400 & 1.13419300 \\ \mathrm{H} & 1.73910300 & 5.21562900 & -2.73687100 \\ \mathrm{H} & 1.17333800 & 7.15837100 & -1.29104800 \\ \mathrm{C} & 3.81346200 & -2.31771000 & -0.65886900 \\ \mathrm{C} & 4.99623700 & -2.60331500 & -1.33983600 \\ \mathrm{C} & 3.18810600 & -3.26050700 & 0.16347400 \\ \mathrm{C} & 5.58457500 & -3.86074500 & -1.16941100 \\ \mathrm{H} & 5.43031400 & -1.84955300 & -1.99054500 \\ \mathrm{C} & 3.78931900 & -4.51110000 & 0.32706300 \\ \mathrm{H} & 2.25513500 & -3.01627900 & 0.66517800 \\ \mathrm{C} & 4.98467400 & -4.81057300 & -0.33689000 \\ \mathrm{H} & 6.50713600 & -4.09858500 & -1.69129400 \\ \mathrm{H} & 3.31990900 & -5.25340000 & 0.96613000 \\ \mathrm{H} & 5.44290000 & -5.78726600 & -0.21068000 \\ \mathrm{O} & 3.84913900 & 0.05622900 & -1.96753400 \\ \mathrm{O} & 1.29008400 & 2.15663300 & 2.49125900 \\ \mathrm{O} & 0.64247600 & -1.10261900 & 1.42501100 \\ \mathrm{C} & -0.32190000 & -1.55757200 & 2.16097200 \\ \mathrm{O} & -1.53499000 & -1.36596200 & 1.95311900 \\ \mathrm{C} & 0.10312000 & -2.35304100 & 3.38561900 \\ \mathrm{H} & -0.66985800 & -3.07732600 & 3.64841400 \\ \mathrm{H} & 1.06189900 & -2.85147900 & 3.22542900 \\ \mathrm{H} & 0.21967400 & -1.65510700 & 4.22241800\end{array}$

\section{P2-INT1}




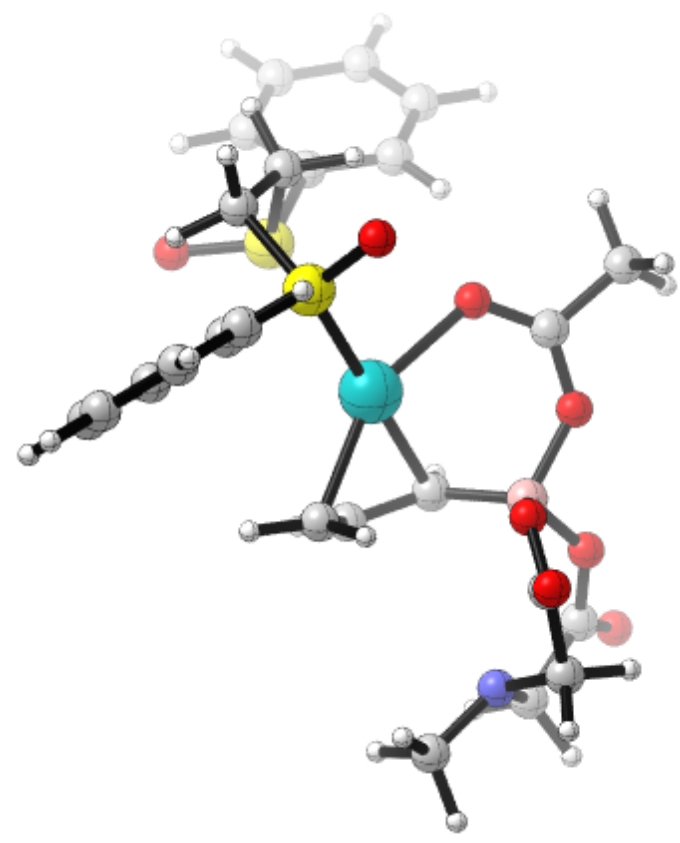

Zero-point correction $=0.495917$ (Hartree/Particle)

Thermal correction to Energy $=0.534663$

Thermal correction to Enthalpy $=0.535607$

Thermal correction to Gibbs Free Energy $=0.417244$

Sum of electronic and zero-point Energies $=-2535.563758$

Sum of electronic and thermal Energies $=-2535.525012$

Sum of electronic and thermal Enthalpies $=-2535.524068$

Sum of electronic and thermal Free Energies $=-2535.642431$

Number of Imaginary Frequencies $=0$

$\begin{array}{lrrr}\mathrm{O} & -3.19511200 & -2.73957900 & 0.28608400 \\ \mathrm{C} & -3.92308100 & -3.24426300 & -0.70963900 \\ \mathrm{O} & -3.98058000 & -4.44127900 & -0.91327400 \\ \mathrm{O} & -2.97253200 & -0.37108600 & 1.06205300 \\ \mathrm{C} & -4.23068500 & 0.07133600 & 1.08489700 \\ \mathrm{O} & -4.54113500 & 1.04517500 & 1.74597300 \\ \mathrm{C} & -4.76859600 & -2.29213600 & -1.56112500 \\ \mathrm{H} & -5.78462400 & -2.72820300 & -1.59155400 \\ \mathrm{C} & -5.25189400 & -0.67464700 & 0.22148500 \\ \mathrm{H} & -5.48468900 & -1.62212400 & 0.71993400 \\ \mathrm{H} & -6.17080100 & -0.07686700 & 0.21598900 \\ \mathrm{~B} & -2.35057300 & -1.55341400 & 0.46561900 \\ \mathrm{~N} & -4.73646100 & -0.91053100 & -1.12807500 \\ \mathrm{C} & -1.44546700 & -1.26708800 & -0.85538700 \\ \mathrm{H} & -0.94363100 & -2.16940100 & -1.21692300 \\ \mathrm{C} & -1.40117400 & -0.20632600 & -1.79371400 \\ \mathrm{H} & -0.92243700 & -0.37887800 & -2.75739800 \\ \mathrm{Pd} & -0.08152700 & 0.24573100 & -0.08891600 \\ \mathrm{C} & 3.51907000 & 0.66139300 & 0.52681600 \\ \mathrm{C} & 2.91198500 & 2.05033300 & 0.37852400\end{array}$




\begin{tabular}{|c|c|c|c|}
\hline $\mathrm{C}$ & -5.26104400 & 0.02367500 & -2.11572400 \\
\hline $\mathrm{H}$ & -5.11653500 & 1.05096900 & -1.76736000 \\
\hline $\mathrm{H}$ & -4.71762900 & -0.09142800 & -3.0595880 \\
\hline $\mathrm{H}$ & -6.33911000 & -0.11825400 & -2.3194720 \\
\hline $\mathrm{H}$ & -4.38003700 & -2.35440100 & -2.5845410 \\
\hline $\mathrm{C}$ & -1.59431800 & 1.13075700 & -1.37944800 \\
\hline $\mathrm{H}$ & -2.29876400 & 1.37540400 & -0.5894530 \\
\hline $\mathrm{H}$ & -1.33766200 & 1.93495600 & -2.0626520 \\
\hline $\mathrm{H}$ & 3.17543200 & 0.13948700 & 1.42473200 \\
\hline $\mathrm{H}$ & 4.61019200 & 0.73769900 & 0.54093900 \\
\hline $\mathrm{H}$ & 3.38862600 & 2.76596100 & 1.05591300 \\
\hline $\mathrm{H}$ & 3.00004900 & 2.40842200 & -0.65147400 \\
\hline S & 3.09678300 & -0.37628400 & -0.96369400 \\
\hline S & 1.13472500 & 2.09646600 & 0.88634100 \\
\hline $\mathrm{C}$ & 0.70354500 & 3.75100600 & 0.26511200 \\
\hline $\mathrm{C}$ & 0.30873400 & 4.69846400 & 1.20779000 \\
\hline $\mathrm{C}$ & 0.73309700 & 4.03808800 & -1.10301500 \\
\hline $\mathrm{C}$ & -0.05408700 & 5.97552600 & 0.76606500 \\
\hline $\mathrm{H}$ & 0.28979600 & 4.43000400 & 2.25942900 \\
\hline $\mathrm{C}$ & 0.37492100 & 5.31838500 & -1.52858000 \\
\hline $\mathrm{H}$ & 1.02910900 & 3.28463500 & -1.8281300 \\
\hline $\mathrm{C}$ & -0.01978000 & 6.28507800 & -0.5955530 \\
\hline $\mathrm{H}$ & -0.36621200 & 6.72336500 & 1.4887650 \\
\hline $\mathrm{H}$ & 0.40164700 & 5.55938000 & -2.58710500 \\
\hline $\mathrm{H}$ & -0.30305600 & 7.27740500 & -0.93364400 \\
\hline $\mathrm{C}$ & 4.24842600 & -1.73942100 & -0.59676100 \\
\hline $\mathrm{C}$ & 5.42409300 & -1.83535800 & -1.34032100 \\
\hline $\mathrm{C}$ & 3.90271500 & -2.69675400 & 0.3620470 \\
\hline $\mathrm{C}$ & 6.29134800 & -2.90470300 & -1.0947720 \\
\hline $\mathrm{H}$ & 5.63673700 & -1.08604100 & -2.0974740 \\
\hline $\mathrm{C}$ & 4.78038900 & -3.75729900 & 0.60062100 \\
\hline $\mathrm{H}$ & 2.96672100 & -2.61572800 & 0.9087040 \\
\hline $\mathrm{C}$ & 5.97241600 & -3.86065900 & -0.1255120 \\
\hline $\mathrm{H}$ & 7.21150900 & -2.99318100 & -1.6651430 \\
\hline $\mathrm{H}$ & 4.52878900 & -4.50710800 & 1.3450150 \\
\hline $\mathrm{H}$ & 6.64674600 & -4.69192400 & 0.0585770 \\
\hline $\mathrm{O}$ & 3.65831600 & 0.40231700 & -2.1472150 \\
\hline $\mathrm{O}$ & 1.11790300 & 2.14871000 & 2.39268700 \\
\hline $\mathrm{O}$ & 0.63782800 & -1.18784800 & 1.3915300 \\
\hline $\mathrm{C}$ & -0.21572100 & -1.91560500 & 1.9620120 \\
\hline $\mathrm{O}$ & -1.44261700 & -2.08318600 & 1.6163330 \\
\hline $\mathrm{C}$ & 0.19007900 & -2.69256600 & 3.1922450 \\
\hline $\mathrm{H}$ & -0.13108900 & -3.73322600 & 3.0991880 \\
\hline $\mathrm{H}$ & 1.26661700 & -2.63018200 & 3.3516930 \\
\hline $\mathrm{H}$ & -0.32791200 & -2.26557400 & 4.0574850 \\
\hline
\end{tabular}

\section{P2-TS2}




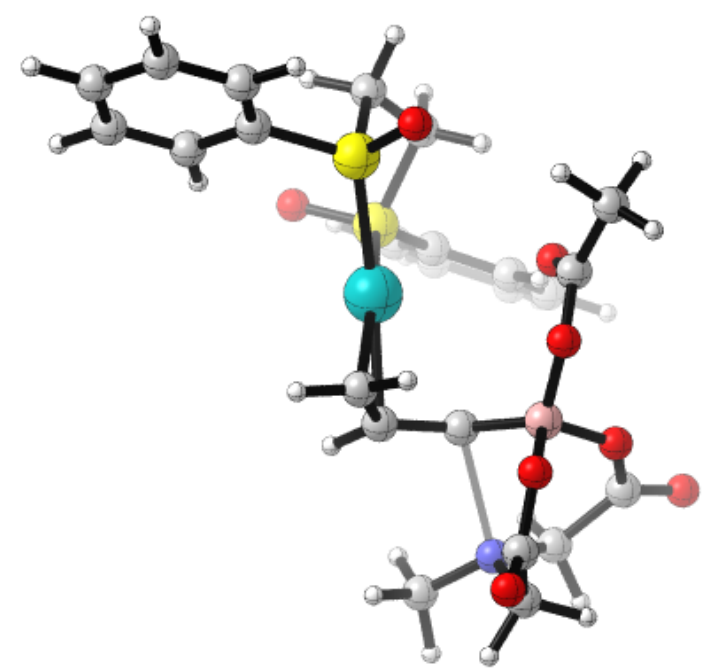

Zero-point correction $=0.497551$ (Hartree/Particle)

Thermal correction to Energy $=0.534852$

Thermal correction to Enthalpy $=0.535796$

Thermal correction to Gibbs Free Energy $=0.424864$

Sum of electronic and zero-point Energies $=-2535.556487$

Sum of electronic and thermal Energies $=-2535.519187$

Sum of electronic and thermal Enthalpies $=-2535.518243$

Sum of electronic and thermal Free Energies $=-2535.629175$

Number of Imaginary Frequencies $=0$

$\begin{array}{lrrr}\mathrm{O} & 3.67541700 & -0.63154300 & 1.23507200 \\ \mathrm{C} & 4.57448800 & 0.11228300 & 0.61911700 \\ \mathrm{O} & 5.35436400 & 0.83491800 & 1.21118900 \\ \mathrm{O} & 2.77834300 & -2.80792400 & 0.48642800 \\ \mathrm{C} & 3.59511300 & -3.32834100 & -0.40606900 \\ \mathrm{O} & 3.69554200 & -4.52685800 & -0.59459800 \\ \mathrm{C} & 4.64955800 & 0.06499600 & -0.91576700 \\ \mathrm{H} & 5.71375900 & 0.05275100 & -1.18858200 \\ \mathrm{C} & 4.51095000 & -2.37160100 & -1.18349900 \\ \mathrm{H} & 5.40754300 & -2.22999600 & -0.56823800 \\ \mathrm{H} & 4.82195300 & -2.89513500 & -2.09012400 \\ \mathrm{~B} & 2.48197000 & -1.37324300 & 0.73378500 \\ \mathrm{~N} & 3.92267800 & -1.05575900 & -1.52177600 \\ \mathrm{C} & 1.90612600 & -0.70883000 & -0.64499900 \\ \mathrm{H} & 2.13858200 & 0.32922400 & -0.86997500 \\ \mathrm{C} & 0.92363500 & -1.29012900 & -1.50819400 \\ \mathrm{H} & 0.81847300 & -0.90782800 & -2.52371100 \\ \mathrm{Pd} & -0.73249000 & -0.40037900 & -0.44739600 \\ \mathrm{C} & -2.03607800 & 2.14549900 & 1.27683500 \\ \mathrm{C} & -3.25127500 & 1.22689900 & 1.23912700 \\ \mathrm{C} & 3.74404500 & -0.89316100 & -2.97019500 \\ \mathrm{H} & 3.13073500 & -1.71151500 & -3.35526900 \\ \mathrm{H} & 3.23626100 & 0.05293300 & -3.17667400 \\ \mathrm{H} & 4.70898200 & -0.89689200 & -3.49764200\end{array}$




$\begin{array}{lrrr}\mathrm{H} & 4.23506100 & 1.00606100 & -1.29292200 \\ \mathrm{C} & 0.05032400 & -2.31794500 & -1.06904300 \\ \mathrm{H} & 0.28613100 & -2.89323100 & -0.17881800 \\ \mathrm{H} & -0.61979900 & -2.79019400 & -1.78226000 \\ \mathrm{H} & -1.28526900 & 1.85288800 & 2.01724800 \\ \mathrm{H} & -2.34781400 & 3.18291700 & 1.42985600 \\ \mathrm{H} & -3.74638500 & 1.16969600 & 2.21300700 \\ \mathrm{H} & -3.96094100 & 1.55264100 & 0.47595400 \\ \mathrm{~S} & -1.20847700 & 2.05471100 & -0.37882200 \\ \mathrm{~S} & -2.78366000 & -0.54844200 & 0.87149000 \\ \mathrm{C} & -4.28314500 & -1.06322800 & -0.01849000 \\ \mathrm{C} & -5.09668800 & -2.02284700 & 0.58048700 \\ \mathrm{C} & -4.55332100 & -0.54701100 & -1.28997300 \\ \mathrm{C} & -6.23129100 & -2.46447700 & -0.10900200 \\ \mathrm{H} & -4.83639700 & -2.40767400 & 1.56175200 \\ \mathrm{C} & -5.69196700 & -0.99467000 & -1.96249300 \\ \mathrm{H} & -3.89729500 & 0.19230900 & -1.74450800 \\ \mathrm{C} & -6.52823600 & -1.95181300 & -1.37442200 \\ \mathrm{H} & -6.87750200 & -3.21069800 & 0.34368500 \\ \mathrm{H} & -5.92219300 & -0.59954700 & -2.94744800 \\ \mathrm{H} & -7.40868400 & -2.29945800 & -1.90686400 \\ \mathrm{C} & -0.04192400 & 3.44766900 & -0.25688700 \\ \mathrm{C} & -0.32052900 & 4.56859400 & -1.04151700 \\ \mathrm{C} & 1.10399900 & 3.36367800 & 0.54111700 \\ \mathrm{C} & 0.56660800 & 5.64857400 & -1.00740500 \\ \mathrm{H} & -1.21141700 & 4.57965200 & -1.66160200 \\ \mathrm{C} & 1.97841000 & 4.45437400 & 0.56190600 \\ \mathrm{H} & 1.30624600 & 2.47693700 & 1.13343200 \\ \mathrm{C} & 1.71209500 & 5.59222500 & -0.20755100 \\ \mathrm{H} & 0.36273900 & 6.52935100 & -1.60940000 \\ \mathrm{H} & 2.87036900 & 4.40689300 & 1.17964400 \\ \mathrm{H} & 2.40043100 & 6.43222000 & -0.18676500 \\ \mathrm{H} & -2.26557500 & 2.44303500 & -1.40115500 \\ \mathrm{O} & -2.75309200 & -1.28028300 & 2.19502400 \\ \mathrm{O} & 0.79557200 & 0.62645500 & 2.04369700 \\ \mathrm{O} & 0.93139000 & -0.50962000 & 2.50331400 \\ \mathrm{H} & 1.57180100 & -1.48537100 & 1.89225400 \\ \mathrm{H} & 0.31035700 & -0.93659200 & 3.81125600 \\ \mathrm{H} & 0.19241400 & -0.06985100 & 4.46424800 \\ \mathrm{H} & & -1.34995100 & 3.59744200 \\ \mathrm{H} & -1.70742900 & 4.29637000\end{array}$

\section{P2-INT2}




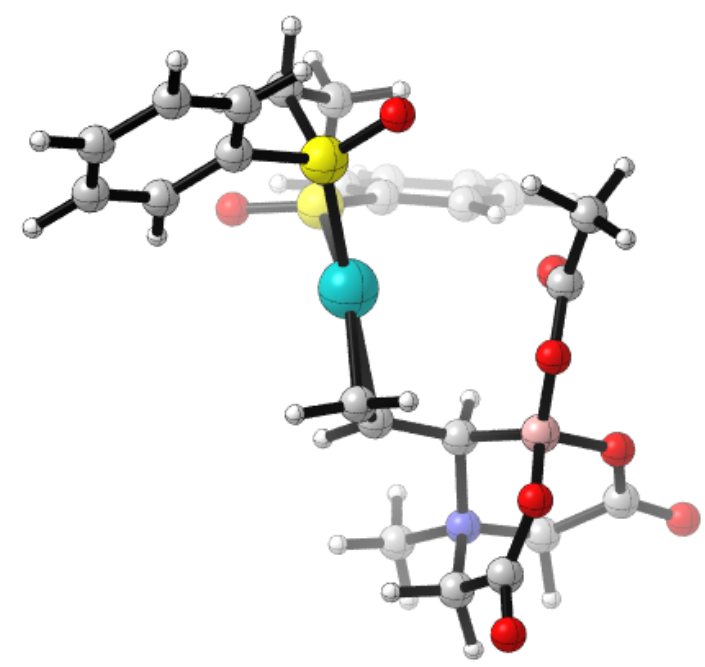

Zero-point correction $=0.500193($ Hartree/Particle $)$

Thermal correction to Energy $=0.537503$

Thermal correction to Enthalpy $=0.538447$

Thermal correction to Gibbs Free Energy $=0.426670$

Sum of electronic and zero-point Energies $=-2535.570414$

Sum of electronic and thermal Energies $=-2535.533103$

Sum of electronic and thermal Enthalpies $=-2535.532159$

Sum of electronic and thermal Free Energies $=-2535.643936$

Number of Imaginary Frequencies $=0$

$\begin{array}{lrrr}\mathrm{O} & 3.95358400 & -0.89428200 & 1.42130400 \\ \mathrm{C} & 4.95843400 & -0.44151500 & 0.72099100 \\ \mathrm{O} & 6.02932000 & -0.09230200 & 1.17902400 \\ \mathrm{O} & 2.97813300 & -2.89540300 & 0.52501100 \\ \mathrm{C} & 3.57965000 & -3.31729200 & -0.55839300 \\ \mathrm{O} & 3.94384200 & -4.46170500 & -0.74698300 \\ \mathrm{C} & 4.77858600 & -0.24412700 & -0.79428600 \\ \mathrm{H} & 5.67963300 & -0.59694800 & -1.29874900 \\ \mathrm{C} & 3.77115600 & -2.34440800 & -1.73737800 \\ \mathrm{H} & 4.76595400 & -2.49522700 & -2.15964100 \\ \mathrm{H} & 3.02985300 & -2.63796900 & -2.48315100 \\ \mathrm{~B} & 2.70090600 & -1.45556900 & 0.82249800 \\ \mathrm{~N} & 3.57459000 & -0.86548500 & -1.48024400 \\ \mathrm{C} & 2.31079500 & -0.65020300 & -0.55854800 \\ \mathrm{H} & 2.32687000 & 0.42927900 & -0.38152700 \\ \mathrm{C} & 1.04714900 & -1.05662400 & -1.29353500 \\ \mathrm{H} & 0.95084000 & -0.70025400 & -2.31853100 \\ \mathrm{Pd} & -0.77367400 & -0.24019200 & -0.41573000 \\ \mathrm{C} & -2.58487700 & 2.40883500 & 0.62714900 \\ \mathrm{C} & -3.64058300 & 1.32044800 & 0.49929400 \\ \mathrm{C} & 3.45532000 & -0.17391000 & -2.81145200 \\ \mathrm{H} & 2.68677400 & -0.65414400 & -3.41254900 \\ \mathrm{H} & 3.19276800 & 0.87118100 & -2.64062500\end{array}$




$\begin{array}{lrrr}\mathrm{H} & 4.41583200 & -0.23975700 & -3.32754800 \\ \mathrm{H} & 4.70307500 & 0.83526100 & -0.94719600 \\ \mathrm{C} & 0.20335700 & -2.11221000 & -0.90920100 \\ \mathrm{H} & 0.40590400 & -2.70131700 & -0.02088000 \\ \mathrm{H} & -0.45272800 & -2.57065400 & -1.64586100 \\ \mathrm{H} & -2.07219600 & 2.38468800 & 1.59359100 \\ \mathrm{H} & -3.02186400 & 3.39822000 & 0.46200900 \\ \mathrm{H} & -4.41731700 & 1.41963300 & 1.26353200 \\ \mathrm{H} & -4.09252800 & 1.32442800 & -0.49637000 \\ \mathrm{~S} & -1.31096500 & 2.17569700 & -0.70601700 \\ \mathrm{~S} & -2.88088800 & -0.35343200 & 0.79031100 \\ \mathrm{C} & -4.22589400 & -1.37461600 & 0.11361300 \\ \mathrm{C} & -5.05269000 & -2.05112200 & 1.00897500 \\ \mathrm{C} & -4.37181500 & -1.50551000 & -1.26994500 \\ \mathrm{C} & -6.07190900 & -2.86311600 & 0.50040100 \\ \mathrm{H} & -4.88469700 & -1.94259700 & 2.07624700 \\ \mathrm{C} & -5.39781600 & -2.31343600 & -1.76429300 \\ \mathrm{H} & -3.69392900 & -0.99362200 & -1.94889800 \\ \mathrm{C} & -6.24634500 & -2.99101600 & -0.88047200 \\ \mathrm{H} & -6.72417300 & -3.39793700 & 1.18454900 \\ \mathrm{H} & -5.52769900 & -2.42165200 & -2.83705200 \\ \mathrm{H} & -7.03718800 & -3.62510500 & -1.27041100 \\ \mathrm{C} & -0.25300100 & 3.60645100 & -0.31377100 \\ \mathrm{C} & -0.37154500 & 4.73762800 & -1.12317500 \\ \mathrm{C} & 0.66860100 & 3.53451900 & 0.73452900 \\ \mathrm{C} & 0.44201600 & 5.84261400 & -0.85564900 \\ \mathrm{H} & -1.08295400 & 4.73482300 & -1.94358200 \\ \mathrm{C} & 1.47138100 & 4.65094100 & 0.98981100 \\ \mathrm{H} & 0.77944000 & 2.63237200 & 1.33125300 \\ \mathrm{C} & 1.35833100 & 5.80069900 & 0.20047200 \\ \mathrm{H} & 0.36110500 & 6.73218200 & -1.47382900 \\ \mathrm{H} & 2.19077800 & 4.61255300 & 1.80250000 \\ \mathrm{H} & 1.98991100 & 6.66081300 & 0.40387800 \\ \mathrm{O} & -2.02450000 & 2.48717400 & -2.01069200 \\ \mathrm{O} & -2.86833300 & -0.54995200 & 2.29449000 \\ \mathrm{O} & 1.43231600 & 0.73074000 & 2.07347300 \\ \mathrm{C} & 1.21170600 & -0.40952000 & 2.46787200 \\ \mathrm{O} & 1.69672600 & -1.49918800 & 1.87244400 \\ \mathrm{H} & 0.33176400 & -0.72614000 & 3.65012100 \\ \mathrm{H} & 0.34738500 & 0.11142100 & 4.34996500 \\ \mathrm{H} & 0.65274700 & -1.64654000 & 4.14184800\end{array}$




\section{Acetic Acid}

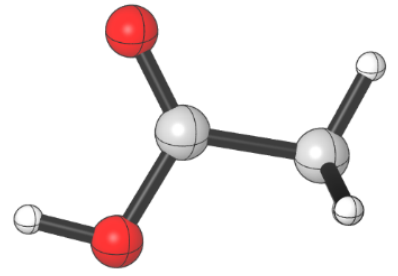

Zero-point correction $=0.061697$ (Hartree/Particle $)$

Thermal correction to Energy $=0.066273$

Thermal correction to Enthalpy $=0.067217$

Thermal correction to Gibbs Free Energy $=0.034478$

Sum of electronic and zero-point Energies $=-229.044130$

Sum of electronic and thermal Energies $=-229.039554$

Sum of electronic and thermal Enthalpies $=-229.038610$

Sum of electronic and thermal Free Energies $=-229.071348$

Number of Imaginary Frequencies $=0$

$\begin{array}{lrrr}\mathrm{O} & -0.79135300 & -1.03873600 & -0.00000700 \\ \mathrm{C} & -0.08829800 & 0.12497300 & -0.00006500 \\ \mathrm{O} & -0.63303800 & 1.20840800 & 0.00001700 \\ \mathrm{C} & 1.39687500 & -0.12114400 & -0.00003500 \\ \mathrm{H} & 1.67748400 & -0.70260600 & 0.88351600 \\ \mathrm{H} & 1.67663300 & -0.70828600 & -0.88001800 \\ \mathrm{H} & 1.92393400 & 0.83216800 & -0.00314300 \\ \mathrm{H} & -1.73438500 & -0.80163500 & 0.00015400\end{array}$

\section{Acetate}

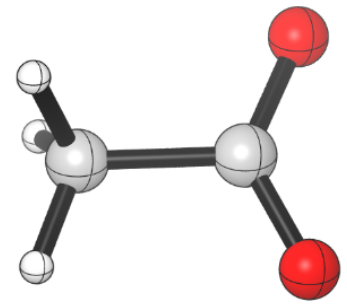

Zero-point correction $=0.047883$ (Hartree/Particle)

Thermal correction to Energy $=0.051464$

Thermal correction to Enthalpy $=0.052408$

Thermal correction to Gibbs Free Energy $=0.021981$

Sum of electronic and zero-point Energies $=-228.495591$

Sum of electronic and thermal Energies $=-228.492010$

Sum of electronic and thermal Enthalpies $=-228.491066$

Sum of electronic and thermal Free Energies $=-228.521493$

Number of Imaginary Frequencies $=0$

$\begin{array}{lrrr}\mathrm{O} & 0.70495800 & 1.16199900 & 0.00001300 \\ \mathrm{C} & 0.20881700 & 0.00116900 & -0.00003800 \\ \mathrm{O} & 0.80410200 & -1.11118900 & 0.00000900\end{array}$




$\begin{array}{lrrr}\mathrm{C} & -1.35365100 & -0.04886100 & -0.00002200 \\ \mathrm{H} & -1.73830500 & 0.47821700 & 0.88295400 \\ \mathrm{H} & -1.73851900 & 0.48034400 & -0.88162100 \\ \mathrm{H} & -1.72664200 & -1.07888800 & -0.00114300\end{array}$

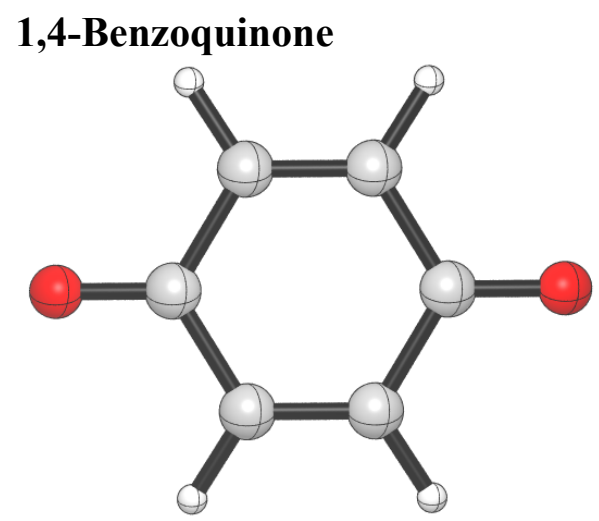

Zero-point correction $=0.084930$ (Hartree/Particle $)$

Thermal correction to Energy $=0.091200$

Thermal correction to Enthalpy $=0.092144$

Thermal correction to Gibbs Free Energy $=0.055658$

Sum of electronic and zero-point Energies $=-381.391568$

Sum of electronic and thermal Energies $=-381.385298$

Sum of electronic and thermal Enthalpies $=-381.384353$

Sum of electronic and thermal Free Energies $=-381.420840$

Number of Imaginary Frequencies $=0$

$\begin{array}{lrrr}\mathrm{C} & 0.00000000 & 0.00000000 & 1.44321100 \\ \mathrm{C} & 0.00000000 & 1.27100200 & 0.67224900 \\ \mathrm{C} & 0.00000000 & 1.27100200 & -0.67224900 \\ \mathrm{C} & 0.00000000 & 0.00000000 & -1.44321100 \\ \mathrm{C} & 0.00000000 & -1.27100200 & -0.67224900 \\ \mathrm{C} & 0.00000000 & -1.27100200 & 0.67224900 \\ \mathrm{H} & 0.00000000 & 2.18467500 & 1.25949800 \\ \mathrm{H} & 0.00000000 & 2.18467500 & -1.25949800 \\ \mathrm{H} & 0.00000000 & -2.18467500 & -1.25949800 \\ \mathrm{H} & 0.00000000 & -2.18467500 & 1.25949800 \\ \mathrm{O} & 0.00000000 & 0.00000000 & -2.67034700 \\ \mathrm{O} & 0.00000000 & 0.00000000 & 2.67034700\end{array}$

Ligand Complex

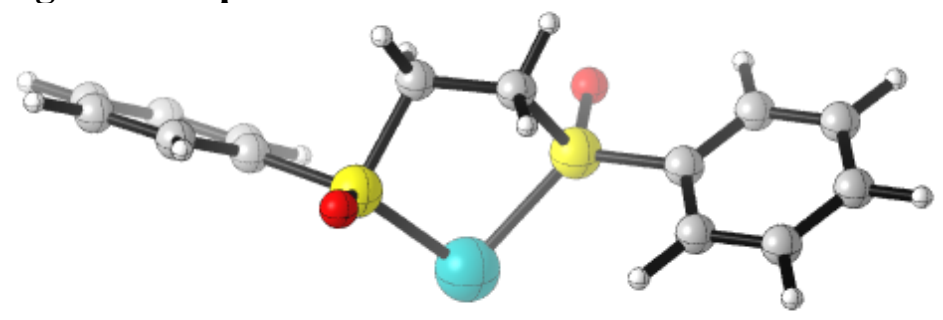

Zero-point correction $=0.248117$ (Hartree/Particle $)$ 
Thermal correction to Energy $=0.267059$

Thermal correction to Enthalpy $=0.268004$

Thermal correction to Gibbs Free Energy $=0.197218$

Sum of electronic and zero-point Energies $=-1615.191052$

Sum of electronic and thermal Energies $=-1615.172110$

Sum of electronic and thermal Enthalpies $=-1615.171165$

Sum of electronic and thermal Free Energies $=-1615.241951$

Number of Imaginary Frequencies $=0$

$\begin{array}{lrrr}\mathrm{Pd} & -0.00002200 & -0.00003000 & 1.53807700 \\ \mathrm{C} & 0.75869100 & 0.06925000 & -1.51482600 \\ \mathrm{C} & -0.75866900 & -0.06904300 & -1.51484700 \\ \mathrm{H} & 1.06951400 & 1.11595500 & -1.45024500 \\ \mathrm{H} & 1.18897100 & -0.38728400 & -2.41234600 \\ \mathrm{H} & -1.18893300 & 0.38757700 & -2.41233200 \\ \mathrm{H} & -1.06949000 & -1.11575600 & -1.45037600 \\ \mathrm{~S} & 1.52335500 & -0.85881100 & -0.08633000 \\ \mathrm{~S} & -1.52337000 & 0.85887900 & -0.08627900 \\ \mathrm{C} & -3.22426000 & 0.22183300 & -0.29244700 \\ \mathrm{C} & -4.18090900 & 1.06253600 & -0.85971300 \\ \mathrm{C} & -3.55229500 & -1.05226000 & 0.17748100 \\ \mathrm{C} & -5.49535700 & 0.60033600 & -0.98469800 \\ \mathrm{H} & -3.88905200 & 2.05742400 & -1.18319900 \\ \mathrm{C} & -4.86592700 & -1.50599000 & 0.04015000 \\ \mathrm{H} & -2.79750700 & -1.67828900 & 0.64850200 \\ \mathrm{C} & -5.83635600 & -0.68060900 & -0.54057300 \\ \mathrm{H} & -6.25178800 & 1.24385000 & -1.42483500 \\ \mathrm{H} & -5.13326000 & -2.49707300 & 0.39505200 \\ \mathrm{H} & -6.85884000 & -1.03402200 & -0.63715400 \\ \mathrm{C} & 3.22427500 & -0.22182600 & -0.29245900 \\ \mathrm{C} & 4.18089800 & -1.06257100 & -0.85970400 \\ \mathrm{C} & 3.55235700 & 1.05225200 & 0.17747800 \\ \mathrm{C} & 5.49537100 & -0.60043100 & -0.98465800 \\ \mathrm{H} & 3.88900100 & -2.05744400 & -1.18320200 \\ \mathrm{C} & 4.86601300 & 1.50592200 & 0.04017600 \\ \mathrm{H} & 2.79759000 & 1.67831700 & 0.64848200 \\ \mathrm{C} & 5.83641800 & 0.68049800 & -0.54052500 \\ \mathrm{H} & 6.25178300 & -1.24398000 & -1.42477900 \\ \mathrm{H} & 5.13338200 & 2.49699400 & 0.39508200 \\ \mathrm{H} & 6.85892000 & 1.03386500 & -0.63708300 \\ \mathrm{O} & 1.53391000 & -2.31638000 & -0.51048000 \\ \mathrm{O} & -1.53398300 & 2.31647900 & -0.51031900\end{array}$




\section{$\underline{\text { Mechanism for Formation of INT-A }}$}

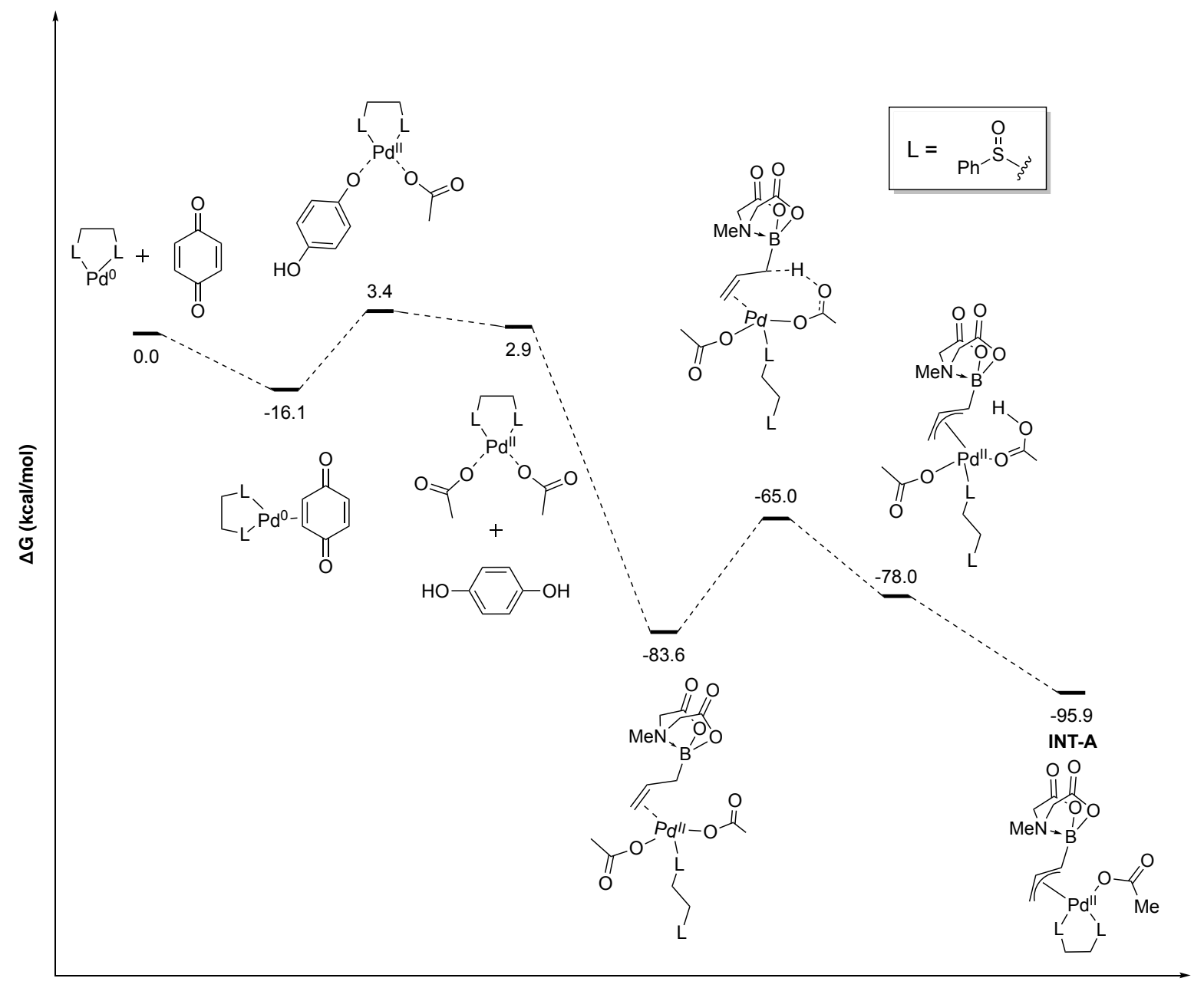

Reaction Coordinate

Figure S-3. Formation of INT-A and re-oxidation of palladium catalyst B3LYP/6$311+\mathrm{G}(2 \mathrm{~d}, 2 \mathrm{p}) / / \mathrm{B} 3 \mathrm{LYP} / 6-31+\mathrm{G}(\mathrm{d}, \mathrm{p})$, solvent: IEFPCM=1,4-dioxane, with the Lan2DZ basis set for Pd level of theory. 


\section{$\underline{\text { NMR Spectra }}$}
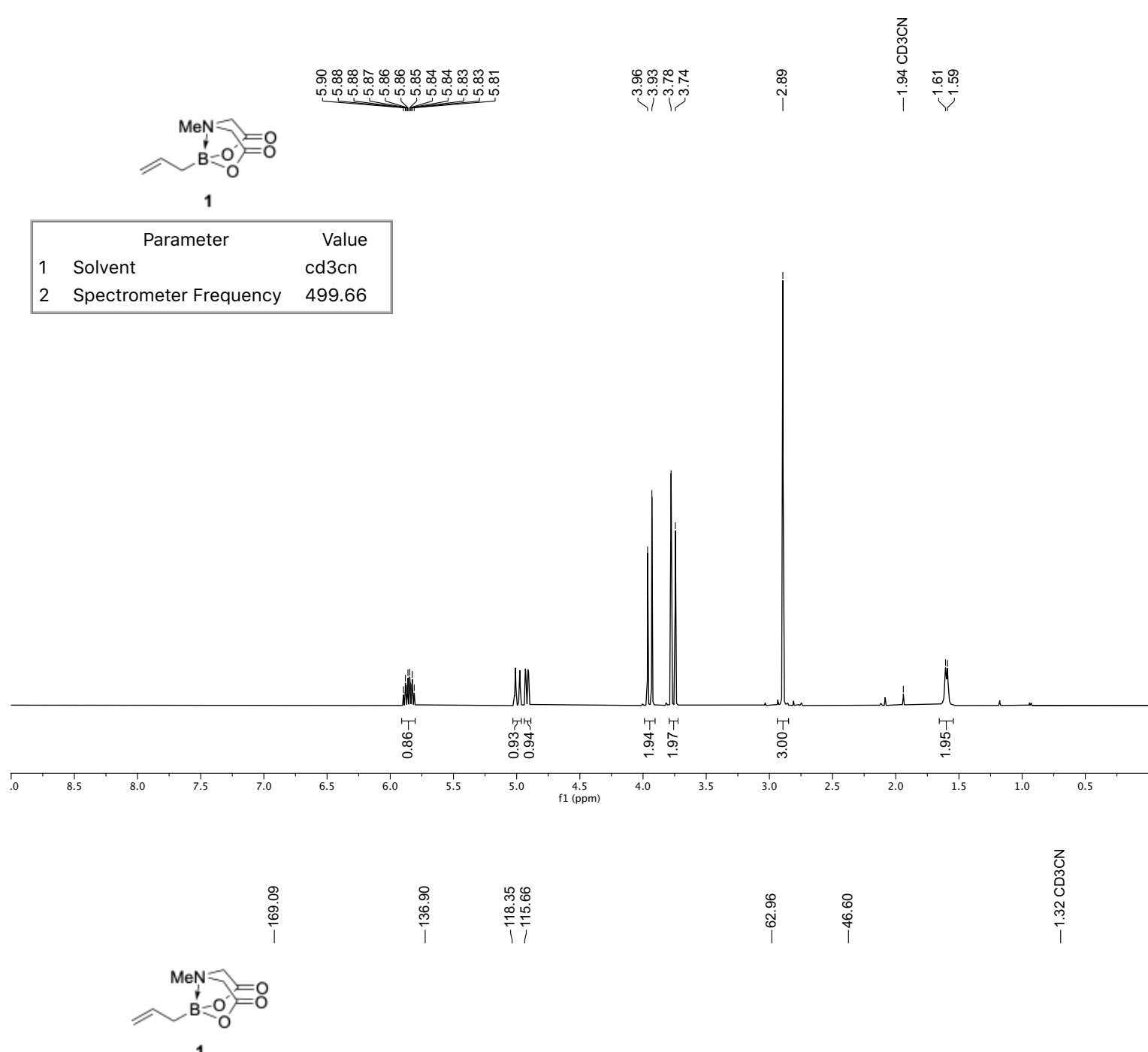

1

\begin{tabular}{|c|c|c|}
\hline & Parameter & Value \\
\hline 1 & Solvent & $\mathrm{cd} 3 \mathrm{cn}$ \\
\hline 2 & Spectrometer Frequency & 125.65 \\
\hline
\end{tabular}
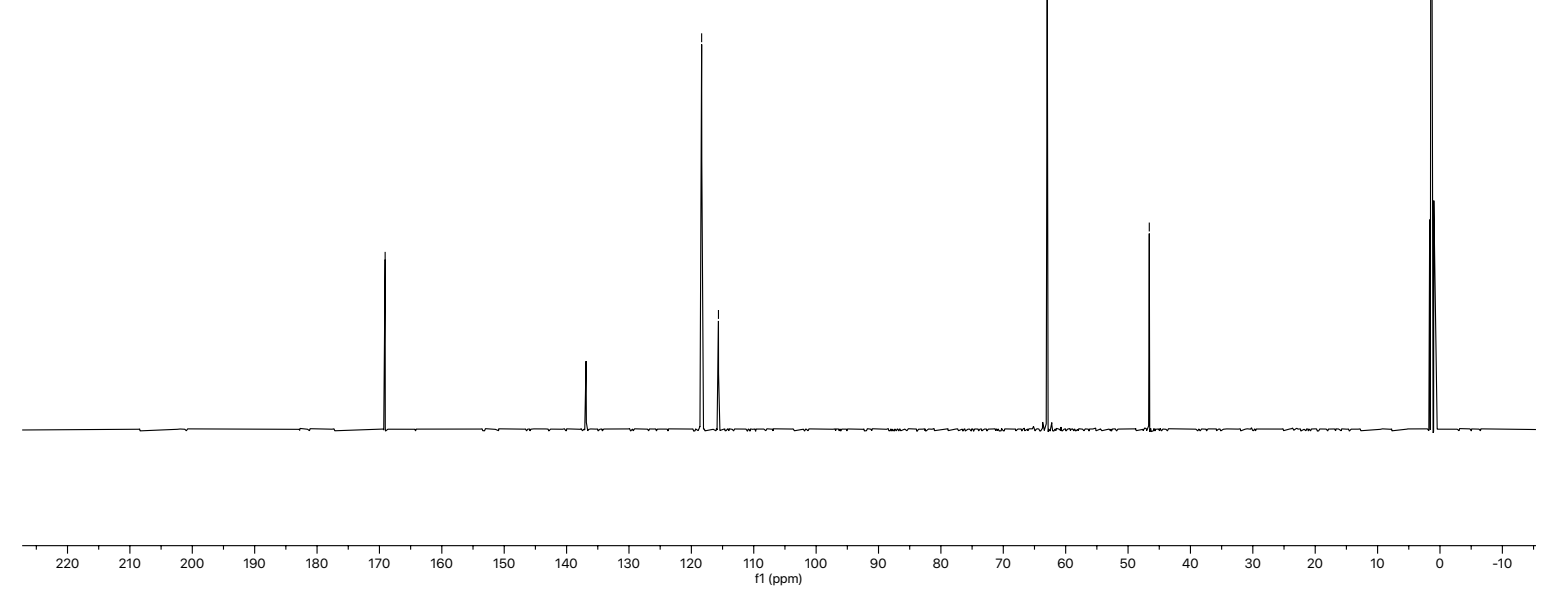

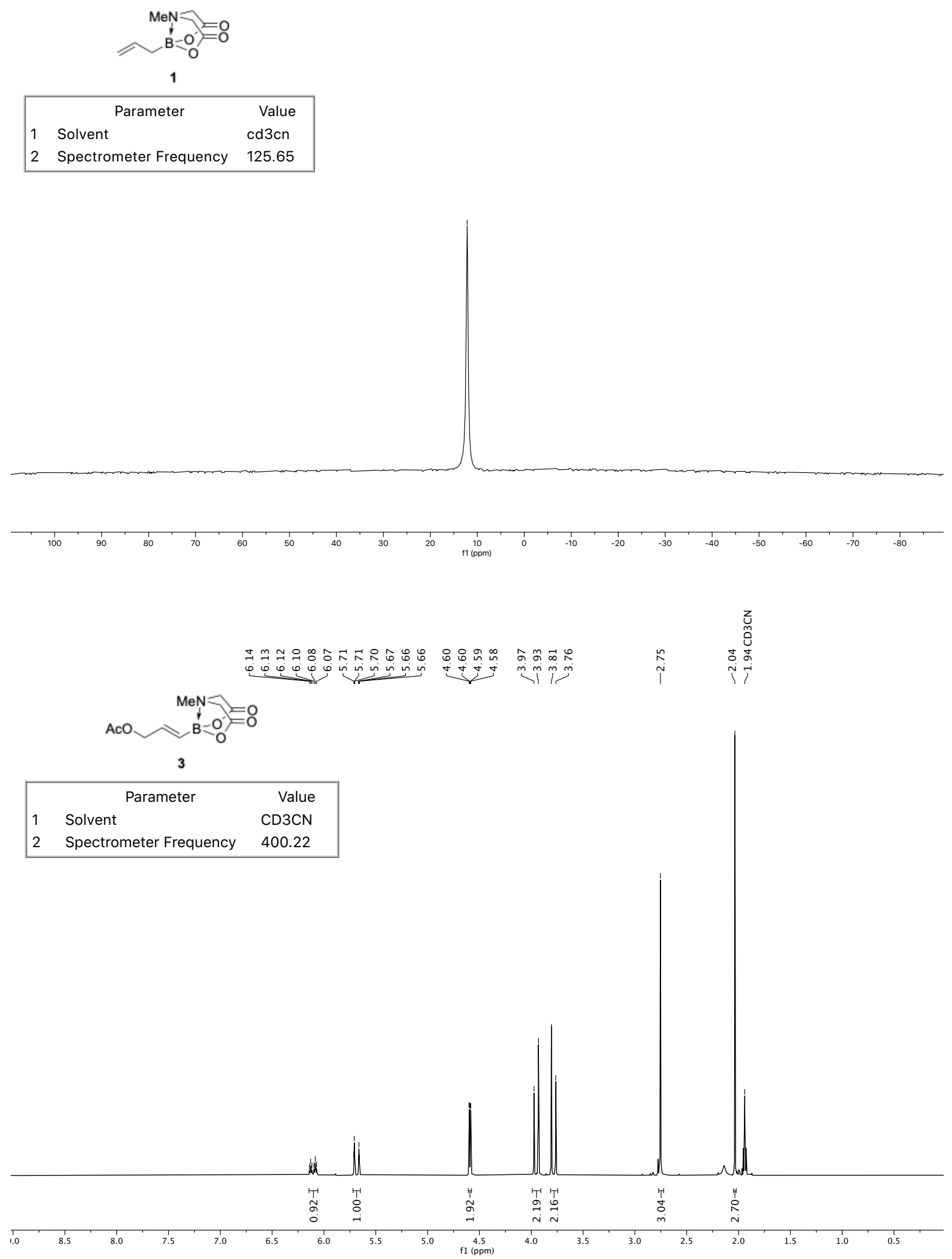

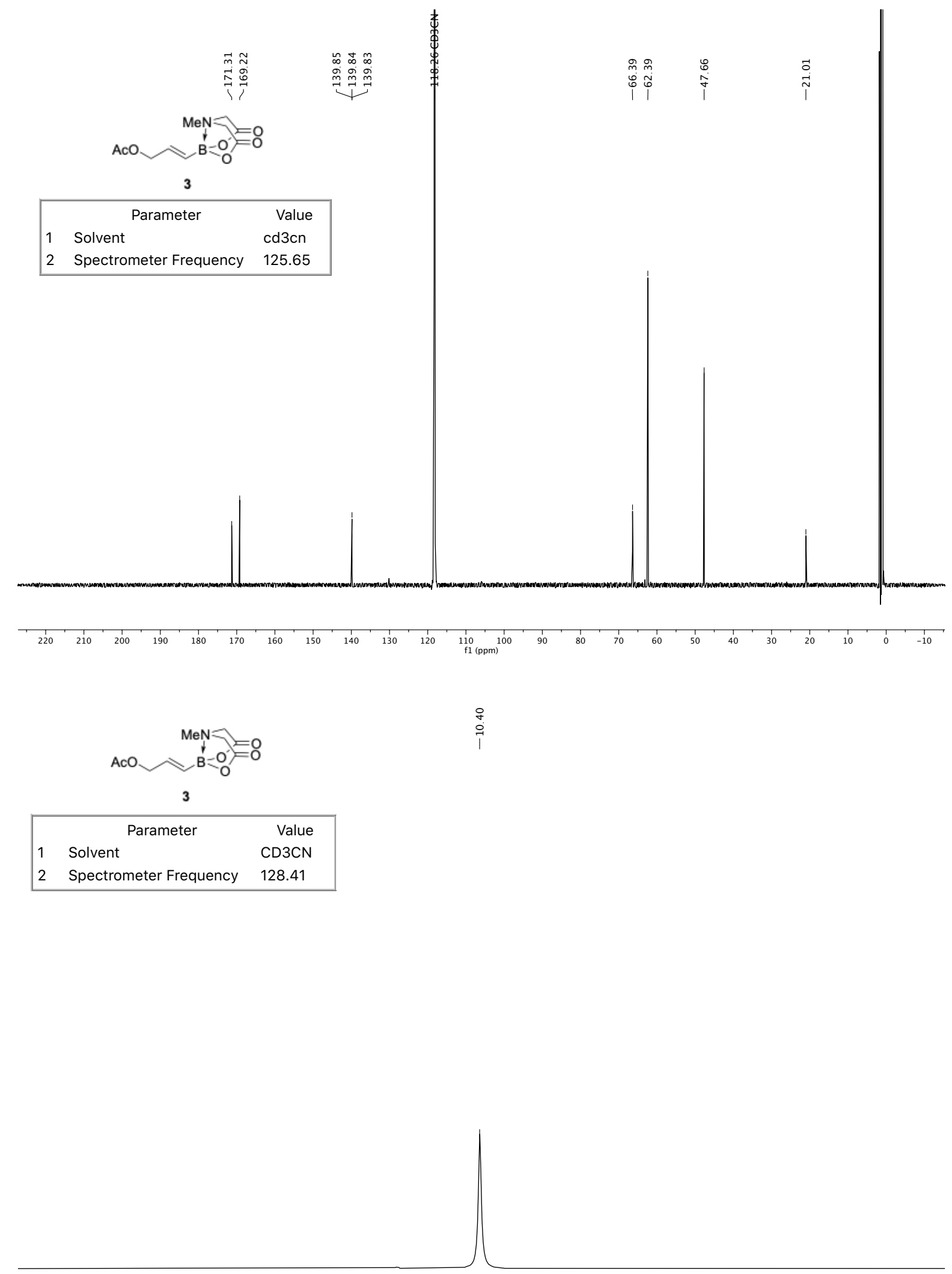

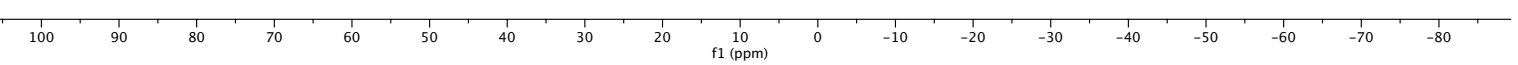




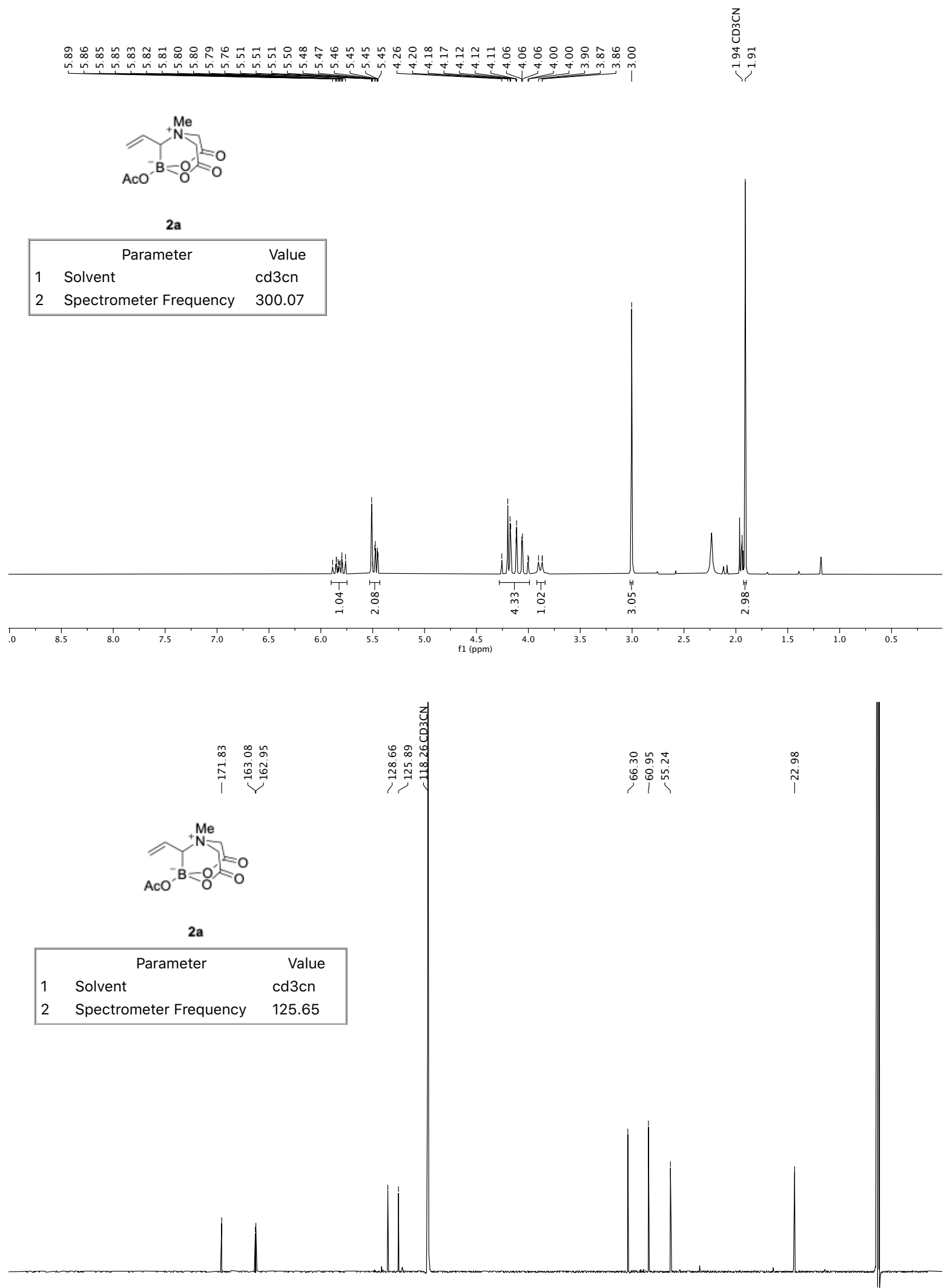

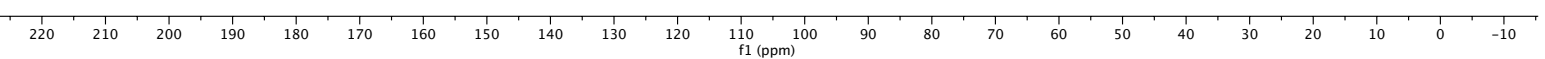




1 Solvent 2
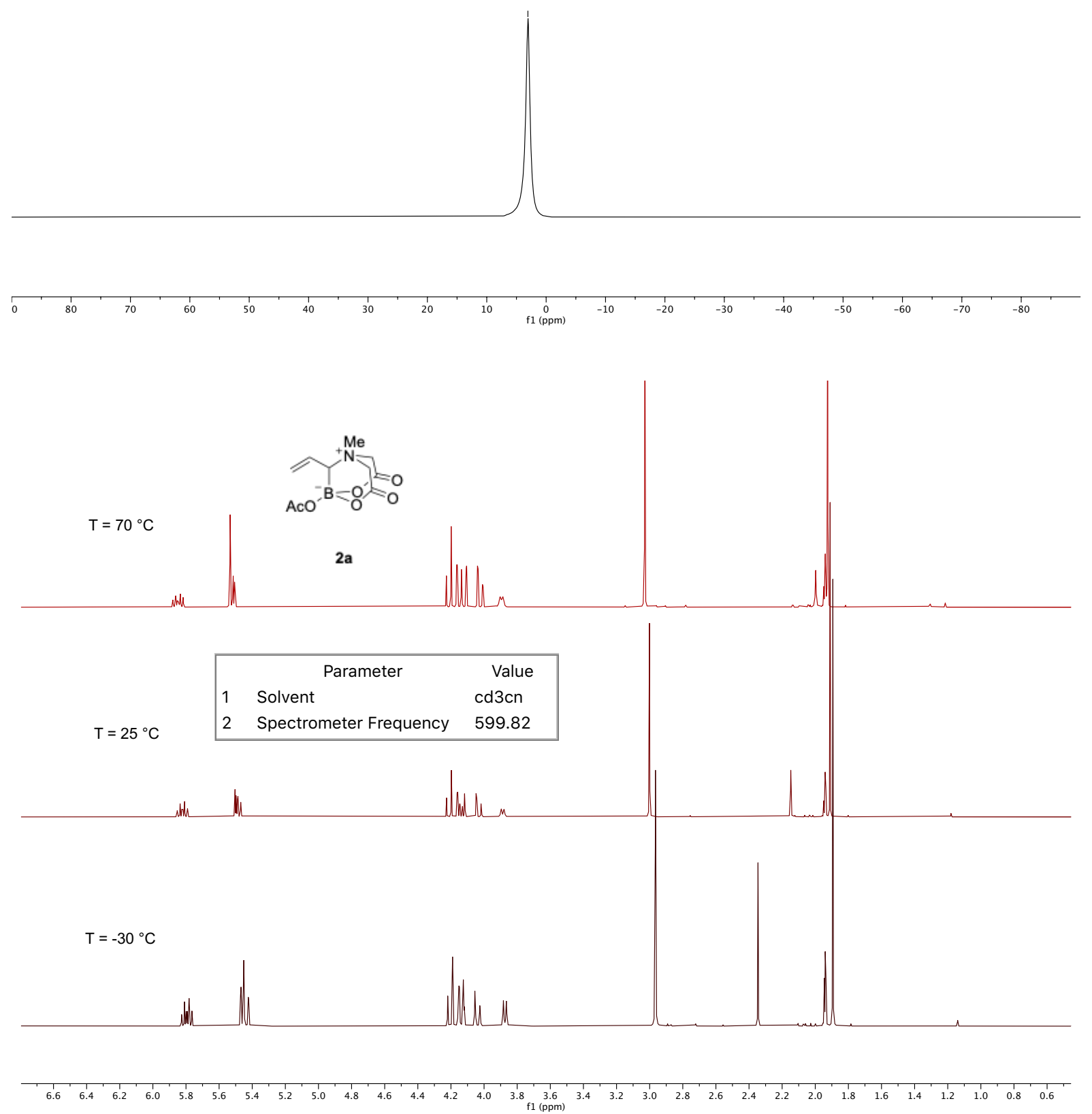

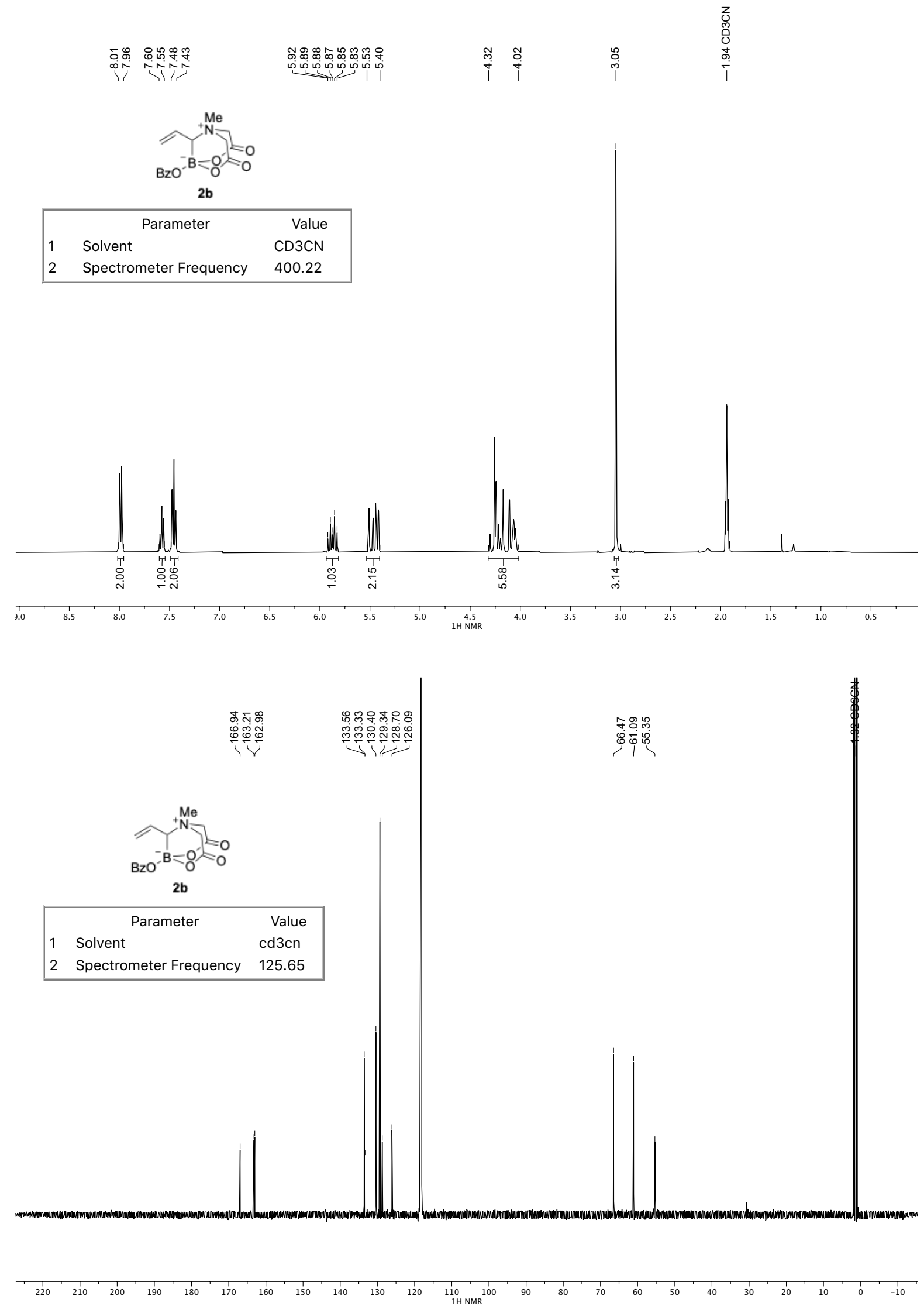


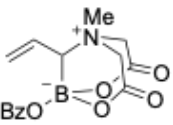

2b

\begin{tabular}{|lll|}
\hline \multicolumn{1}{|c}{ Parameter } & Value \\
1 & Solvent & $\mathrm{CD} 3 \mathrm{CN}$ \\
2 & Spectrometer Frequency & 128.41 \\
\hline
\end{tabular}
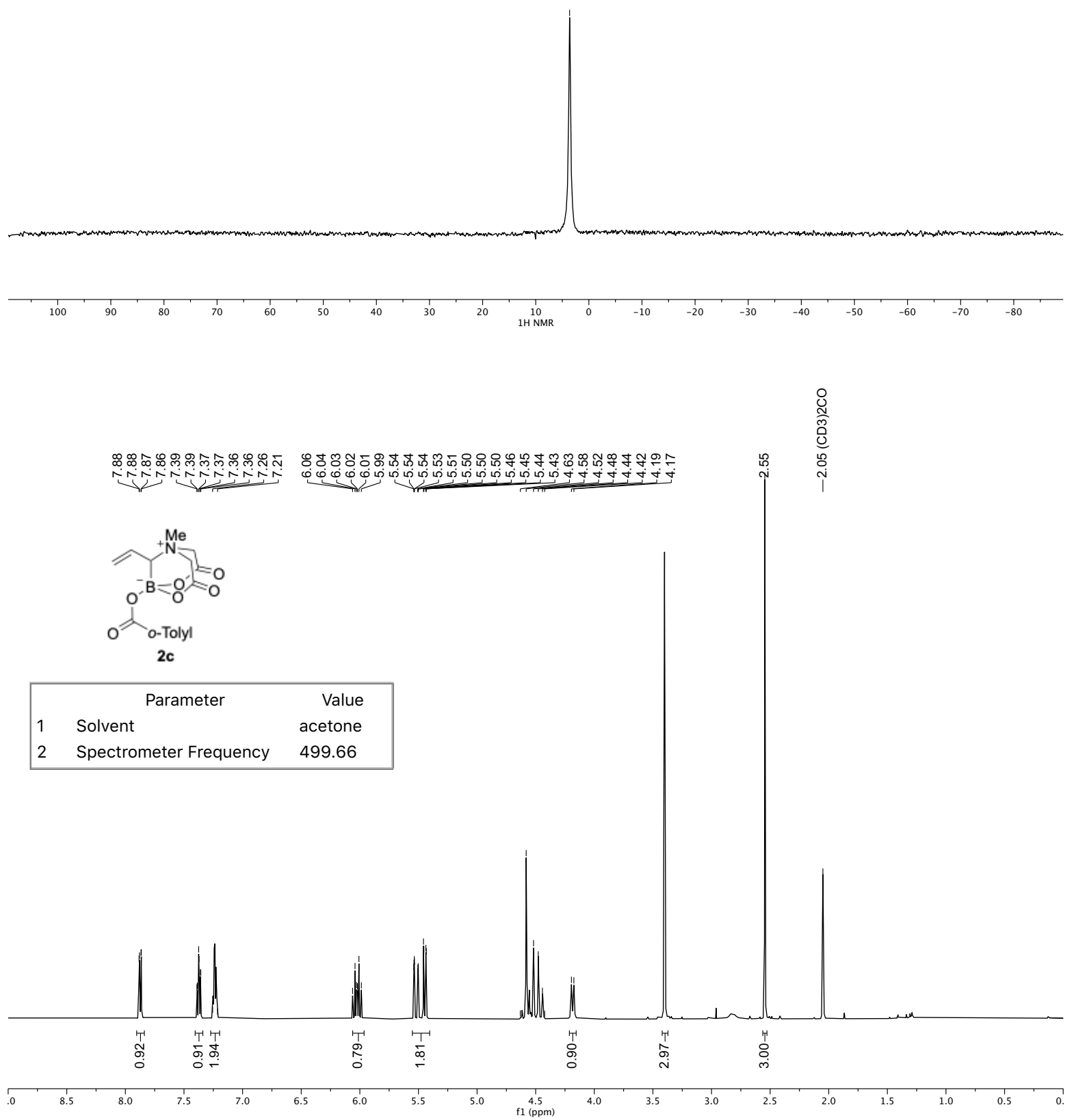

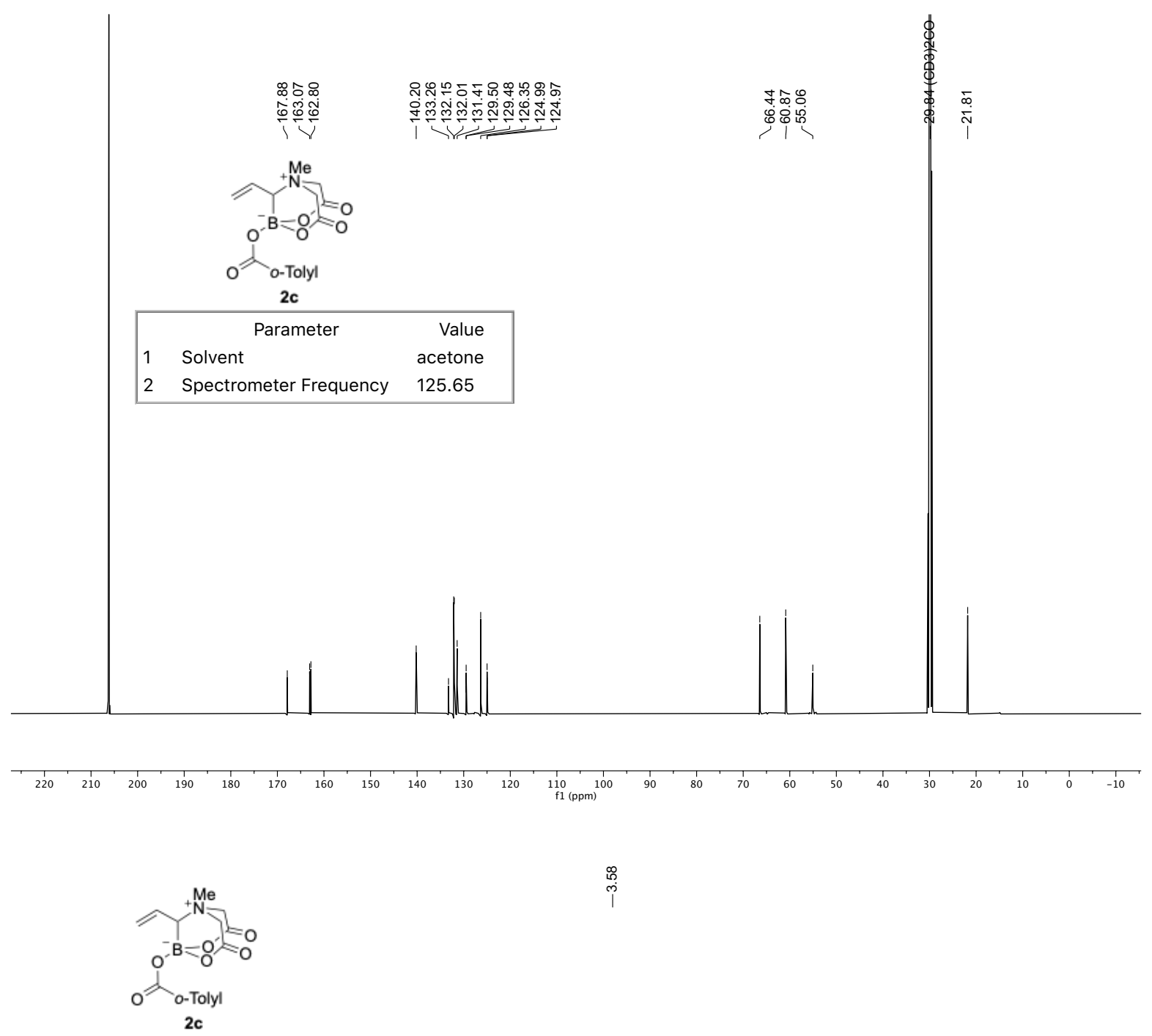

\begin{tabular}{|c|c|c|}
\hline & Parameter & Value \\
\hline 1 & Solvent & $\mathrm{CD} 3 \mathrm{CN}$ \\
\hline 2 & Spectrometer Frequency & 128.41 \\
\hline
\end{tabular}

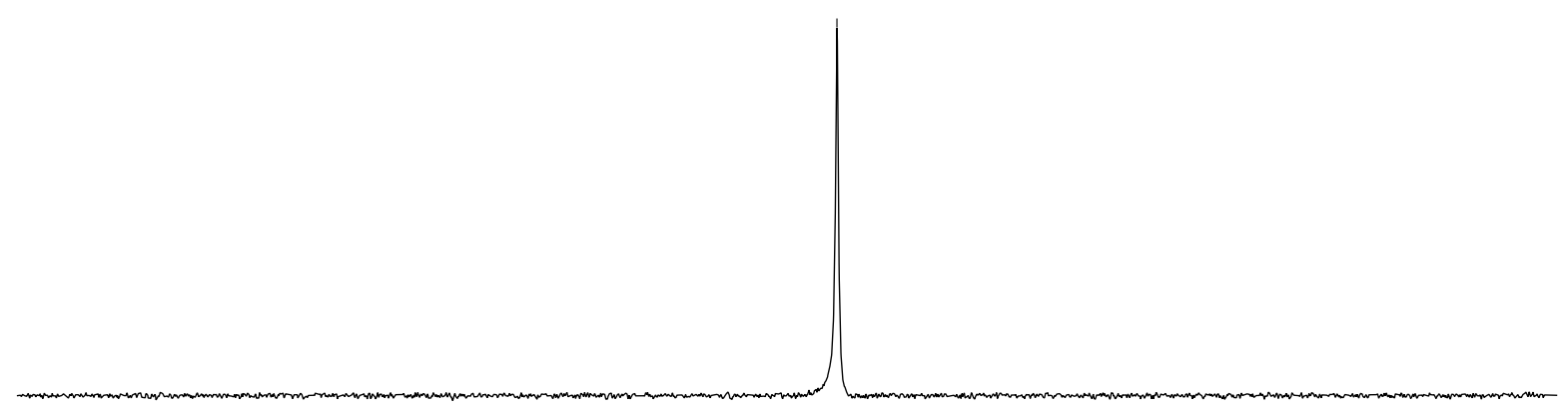



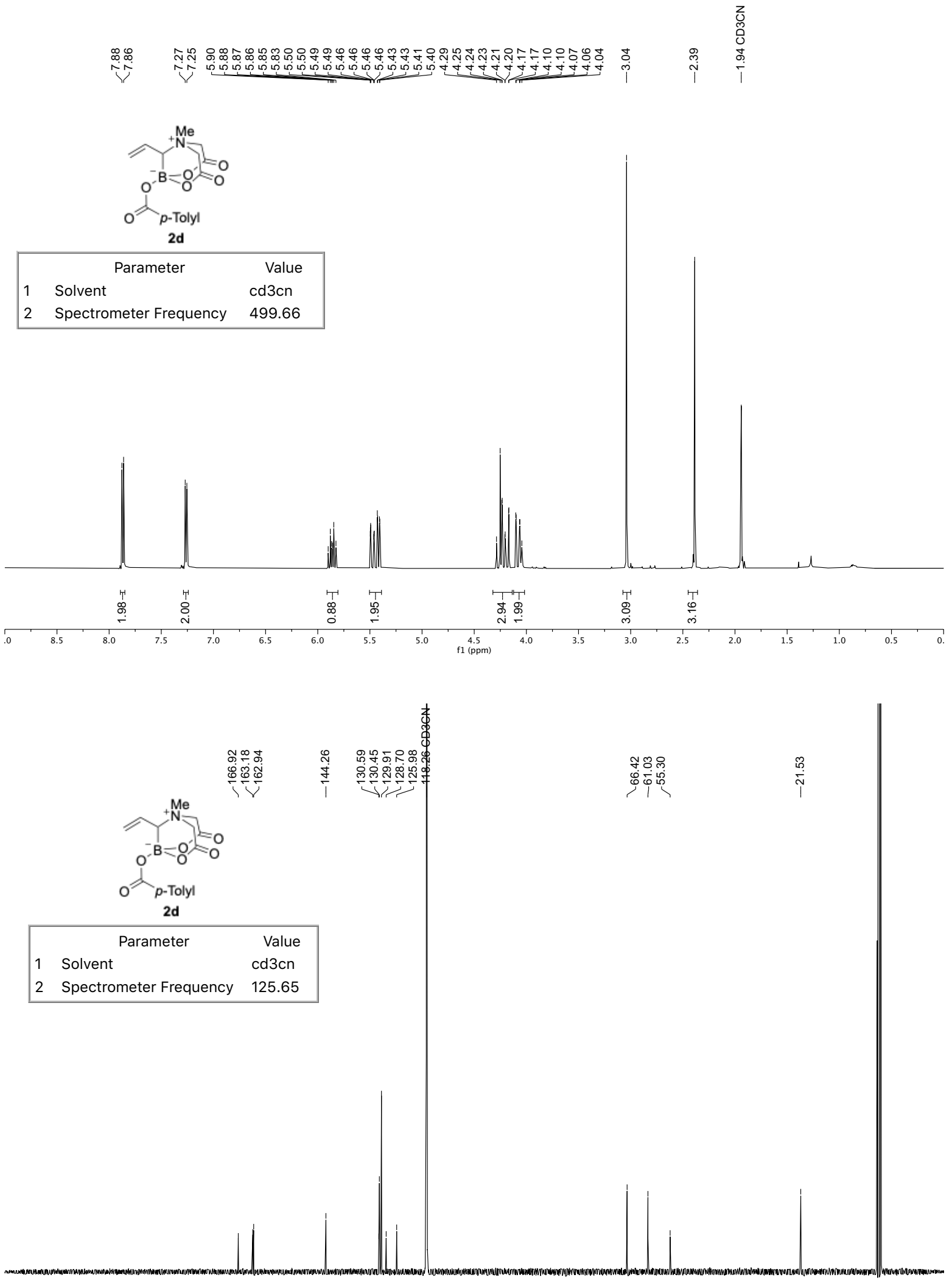

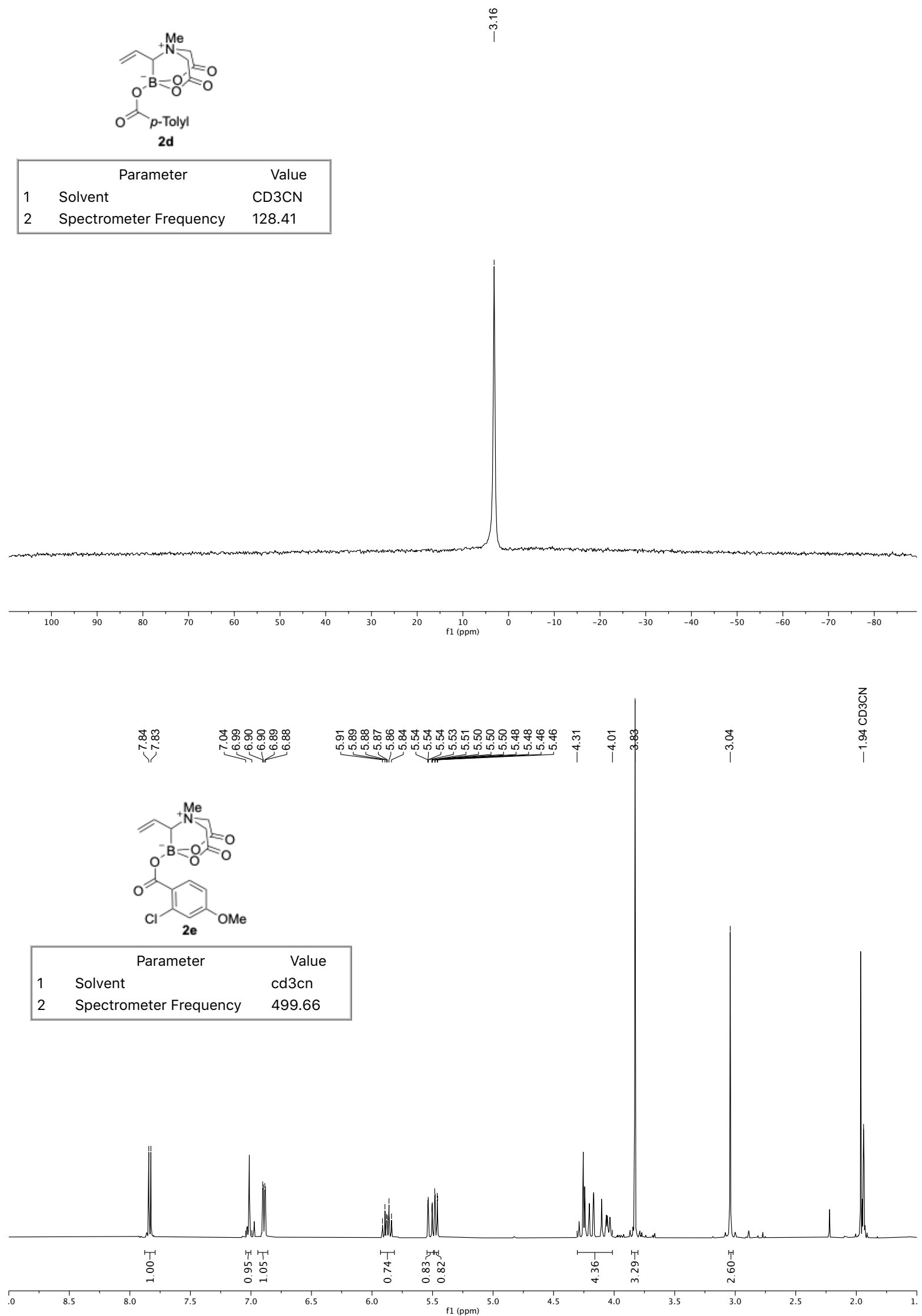

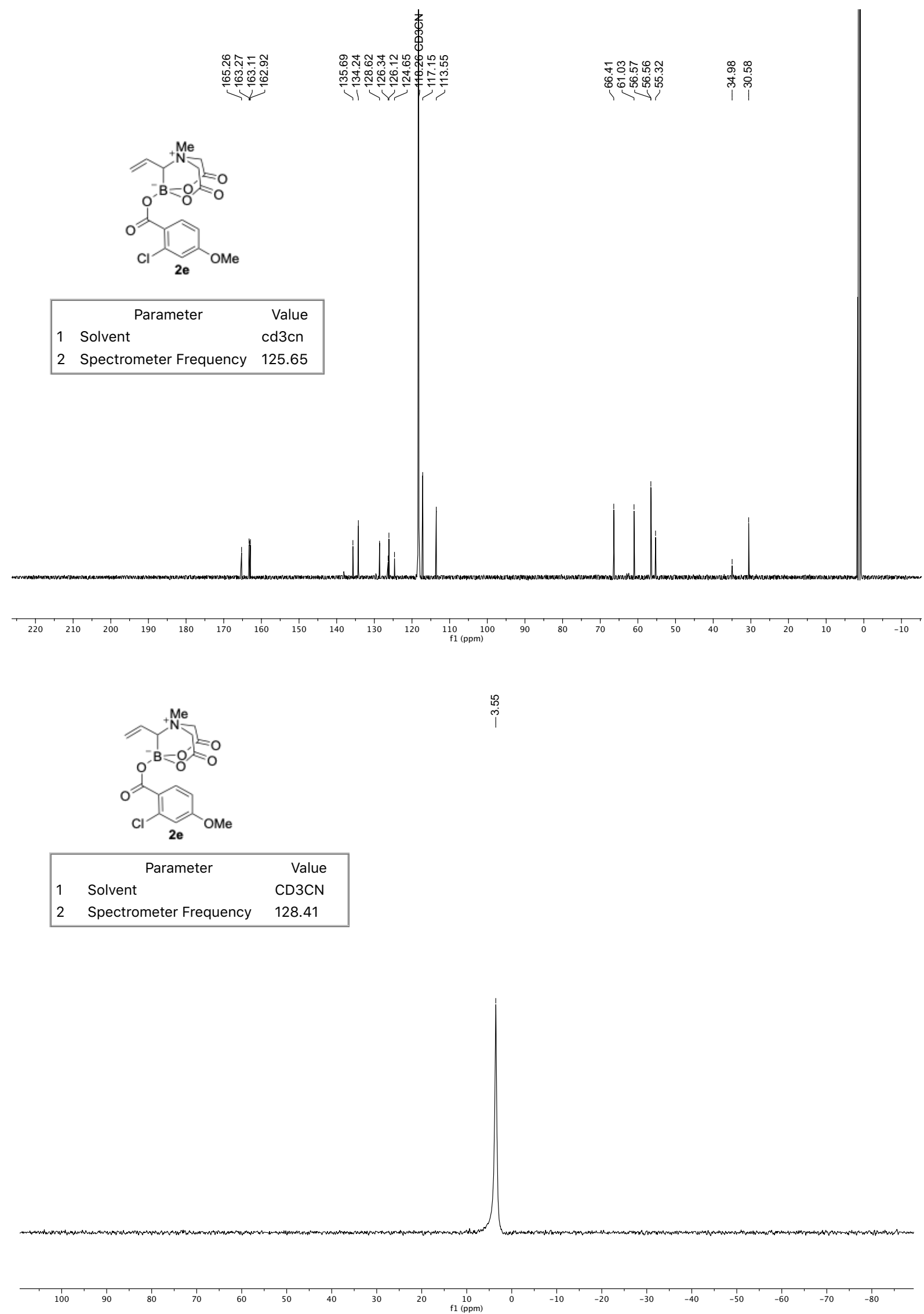

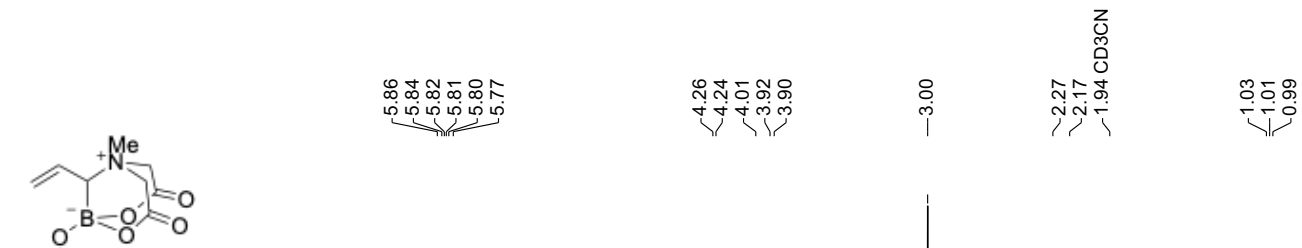

o $\mathrm{Et}$

2f

\begin{tabular}{|lc|}
\hline \multicolumn{1}{|c|}{ Parameter } & Value \\
1 Solvent & CD3CN \\
2 Spectrometer Frequency & 400.22 \\
\hline
\end{tabular}
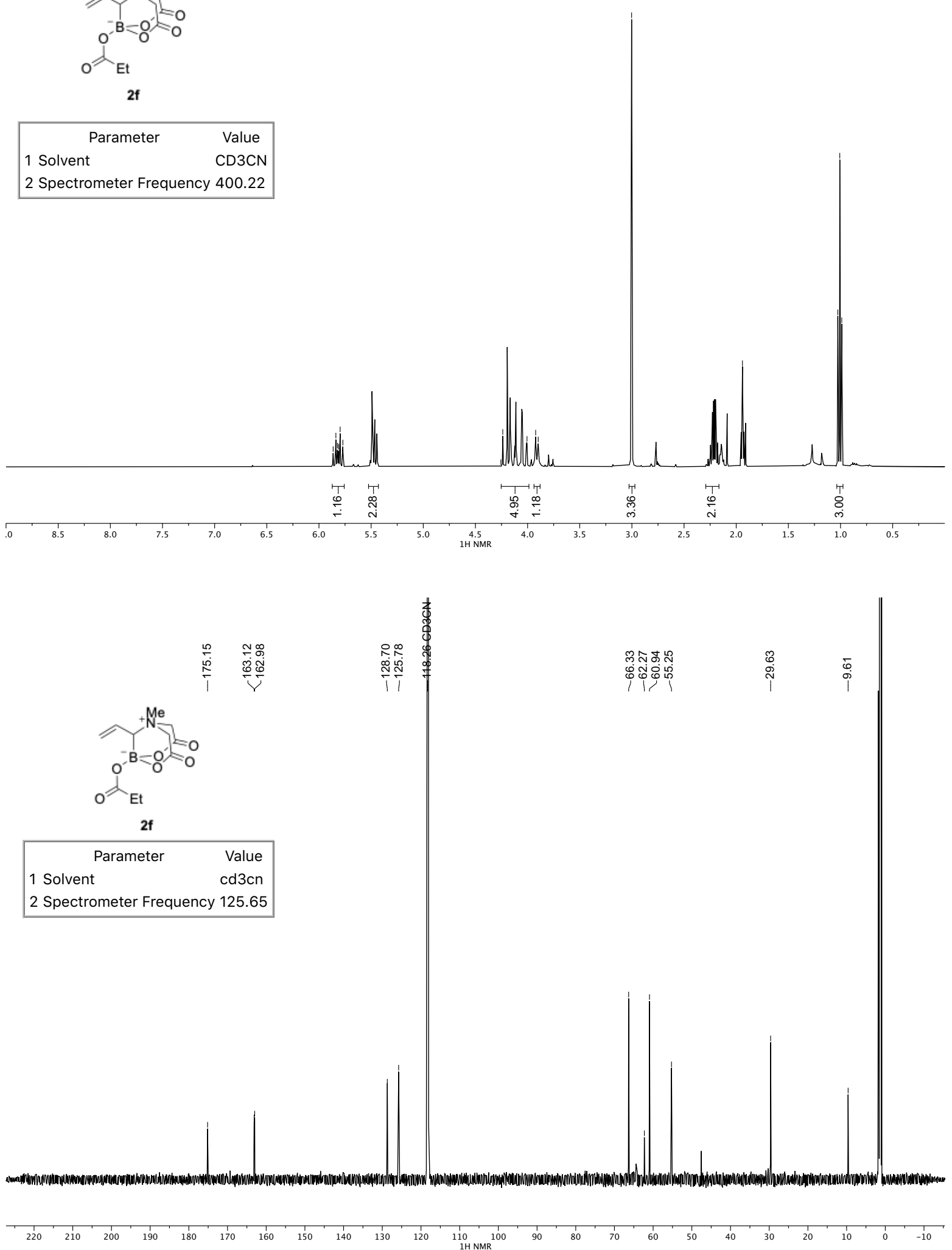


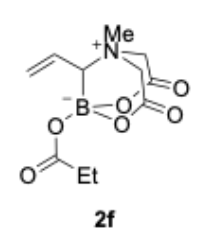

\begin{tabular}{|cc|}
\hline \multicolumn{1}{|c|}{ Parameter } & \multicolumn{1}{c|}{ Value } \\
1 Solvent & $\mathrm{CD} 3 \mathrm{CN}$ \\
2 Spectrometer Frequency & 128.41 \\
\hline
\end{tabular}
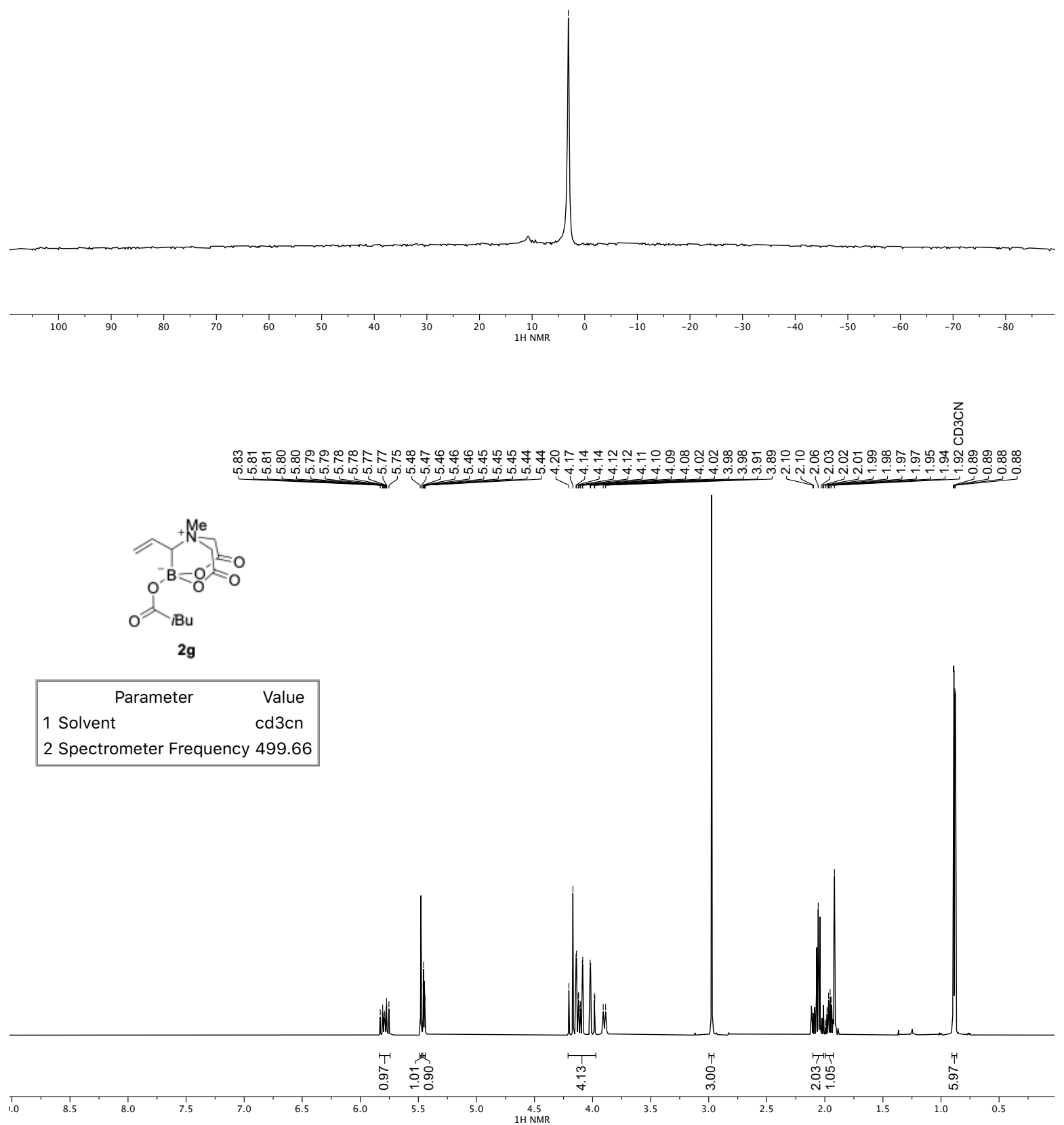

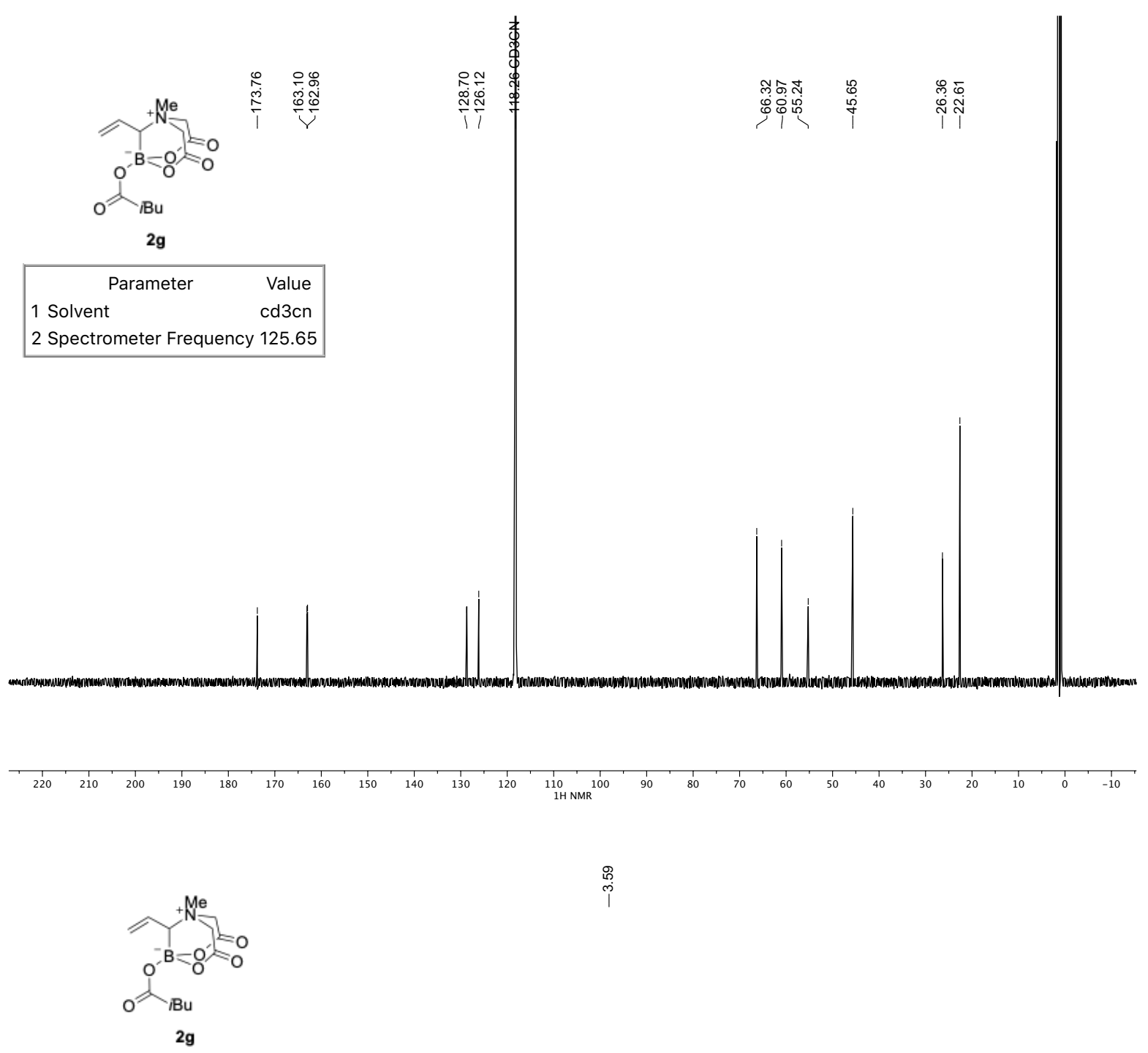

\begin{tabular}{|cr|}
\hline \multicolumn{1}{|c|}{ Parameter } & \multicolumn{1}{c|}{ Value } \\
1 Solvent & $\mathrm{CD} 3 \mathrm{CN}$ \\
2 Spectrometer Frequency & 128.41 \\
\hline
\end{tabular}

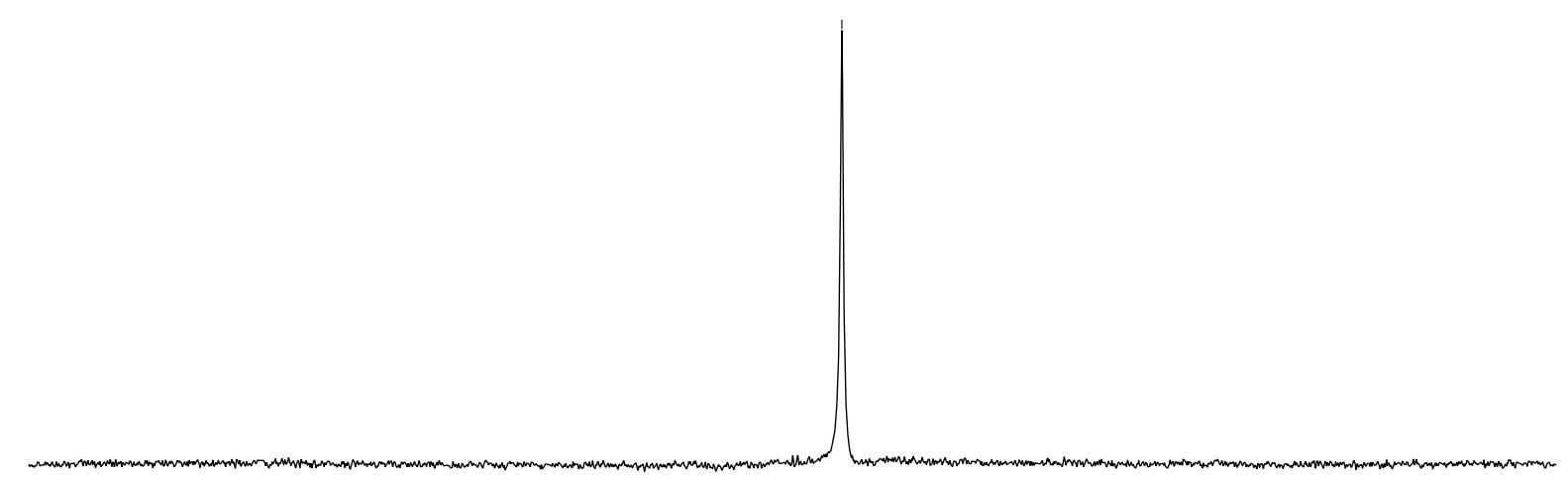



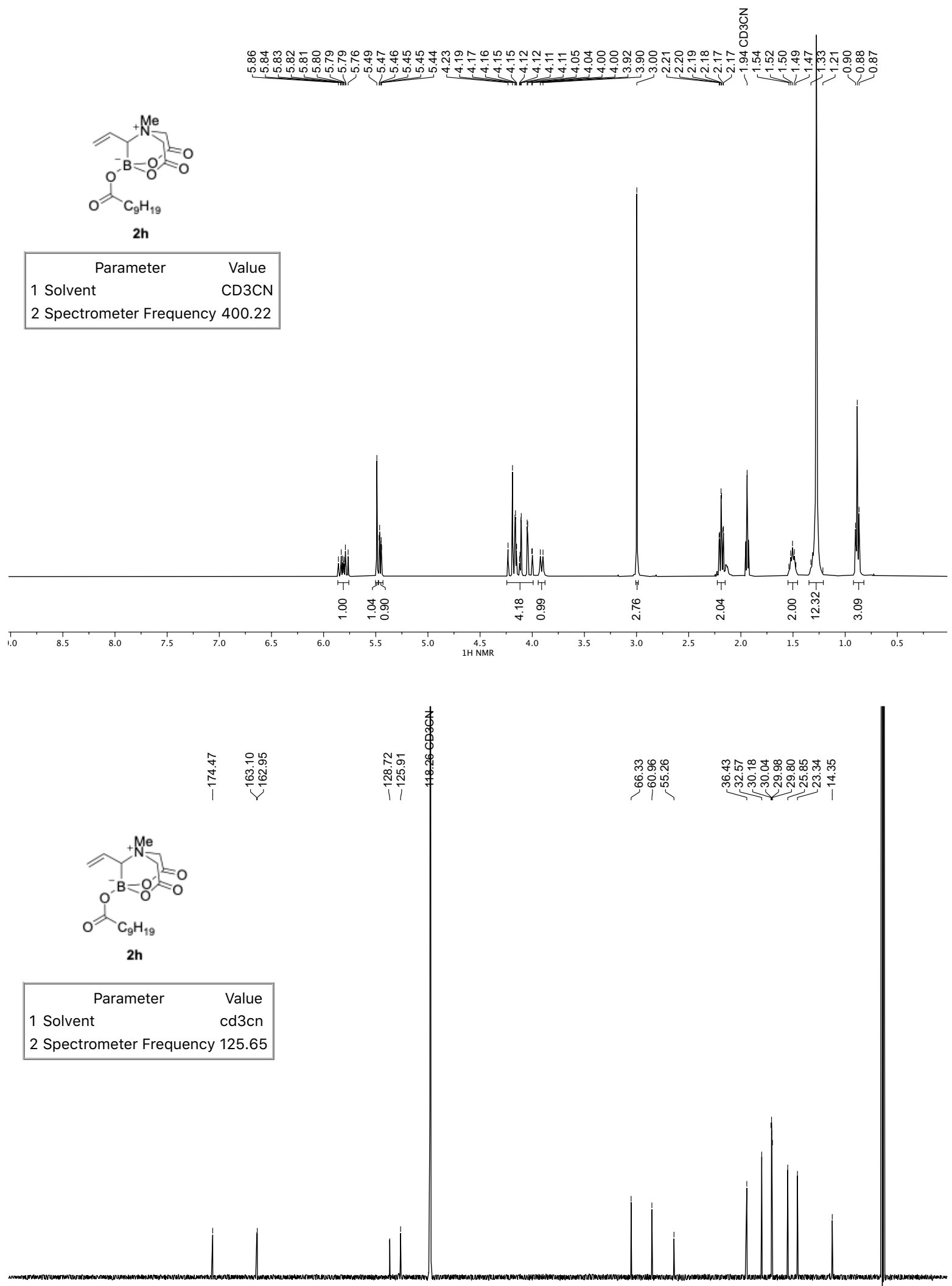

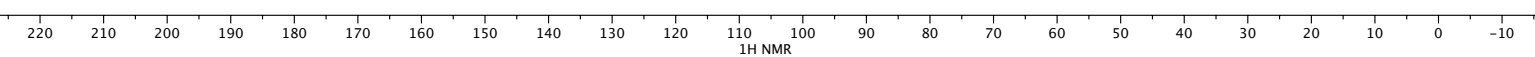




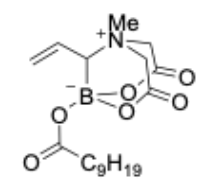

2h

\begin{tabular}{|cc|}
\hline \multicolumn{1}{|c|}{ Parameter } & \multicolumn{1}{c|}{ Value } \\
1 Solvent & CD $3 C N$ \\
2 Spectrometer Frequency & 128.41 \\
\hline
\end{tabular}
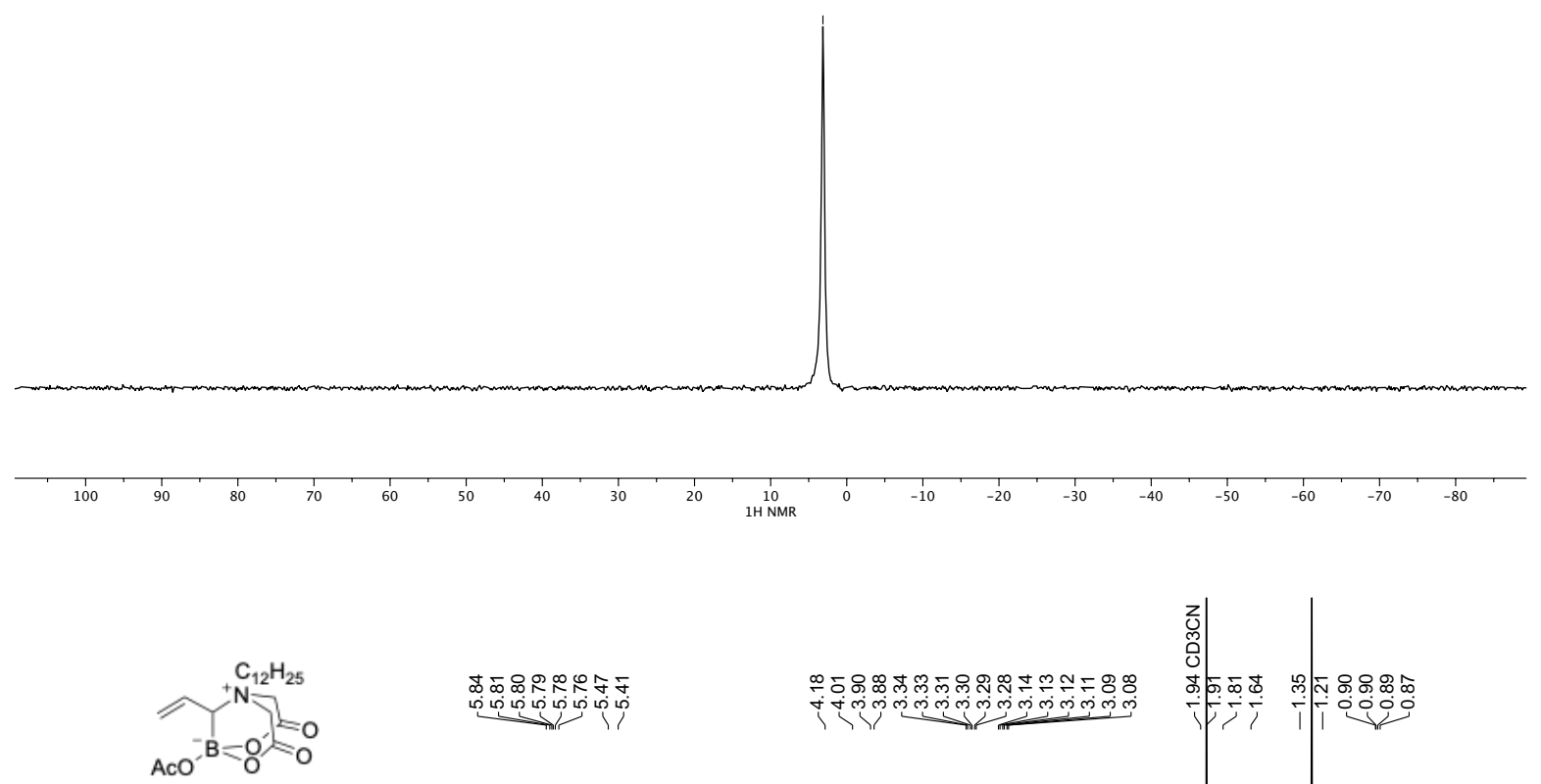

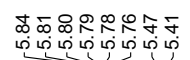

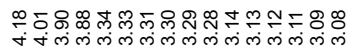
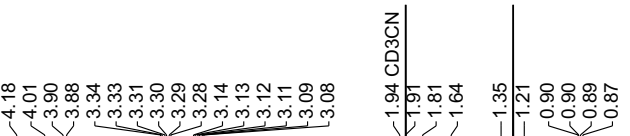

$2 \mathrm{i}$

Parameter

1 Solvent

2 Spectrometer Frequency 499.66

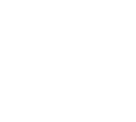



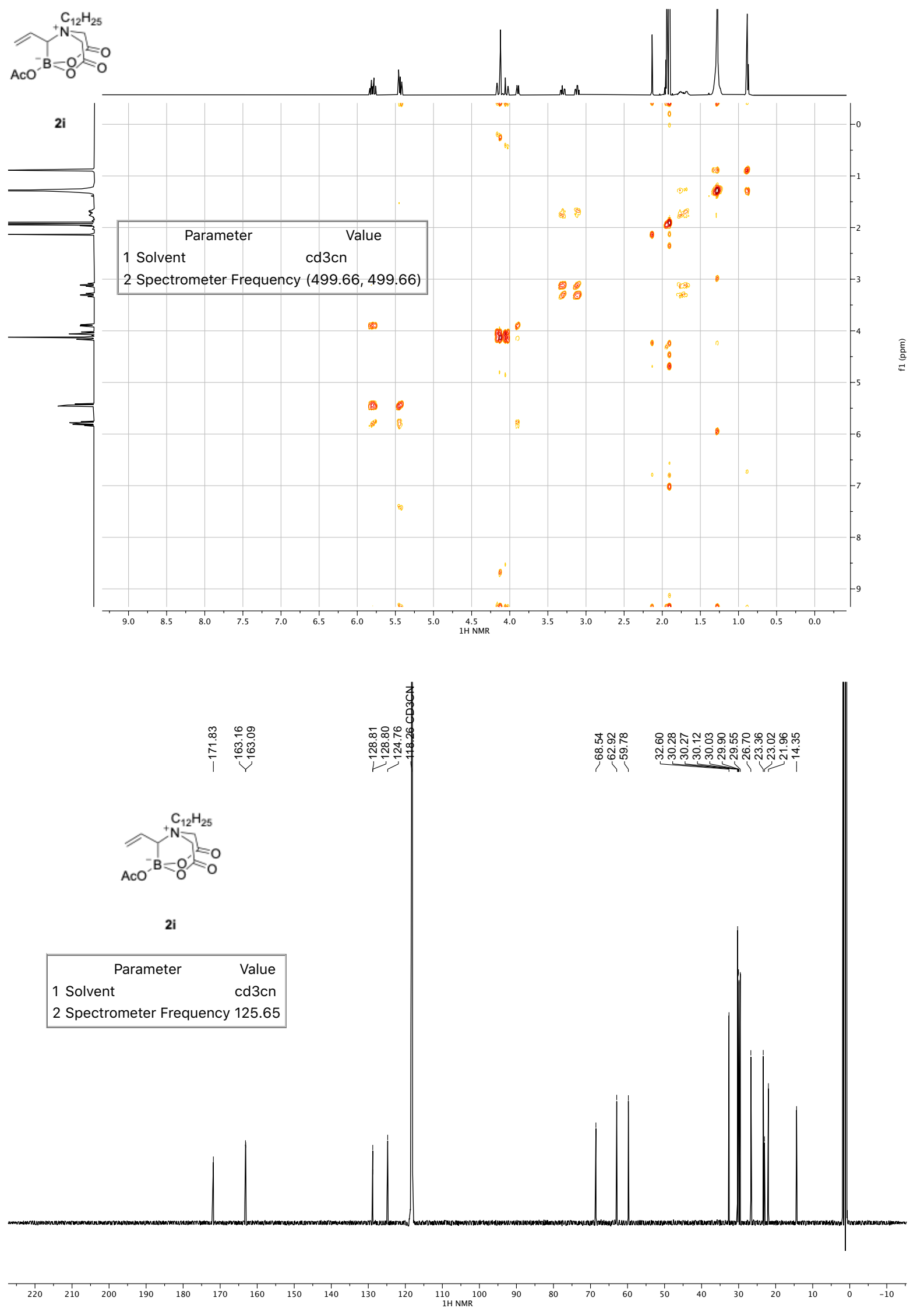


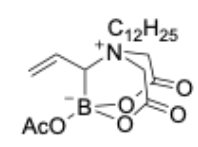

$2 i$

\begin{tabular}{|cc|}
\hline \multicolumn{1}{|c|}{ Parameter } & \multicolumn{1}{c|}{ Value } \\
1 Solvent & CD3CN \\
2 Spectrometer Frequency & 128.41 \\
\hline
\end{tabular}
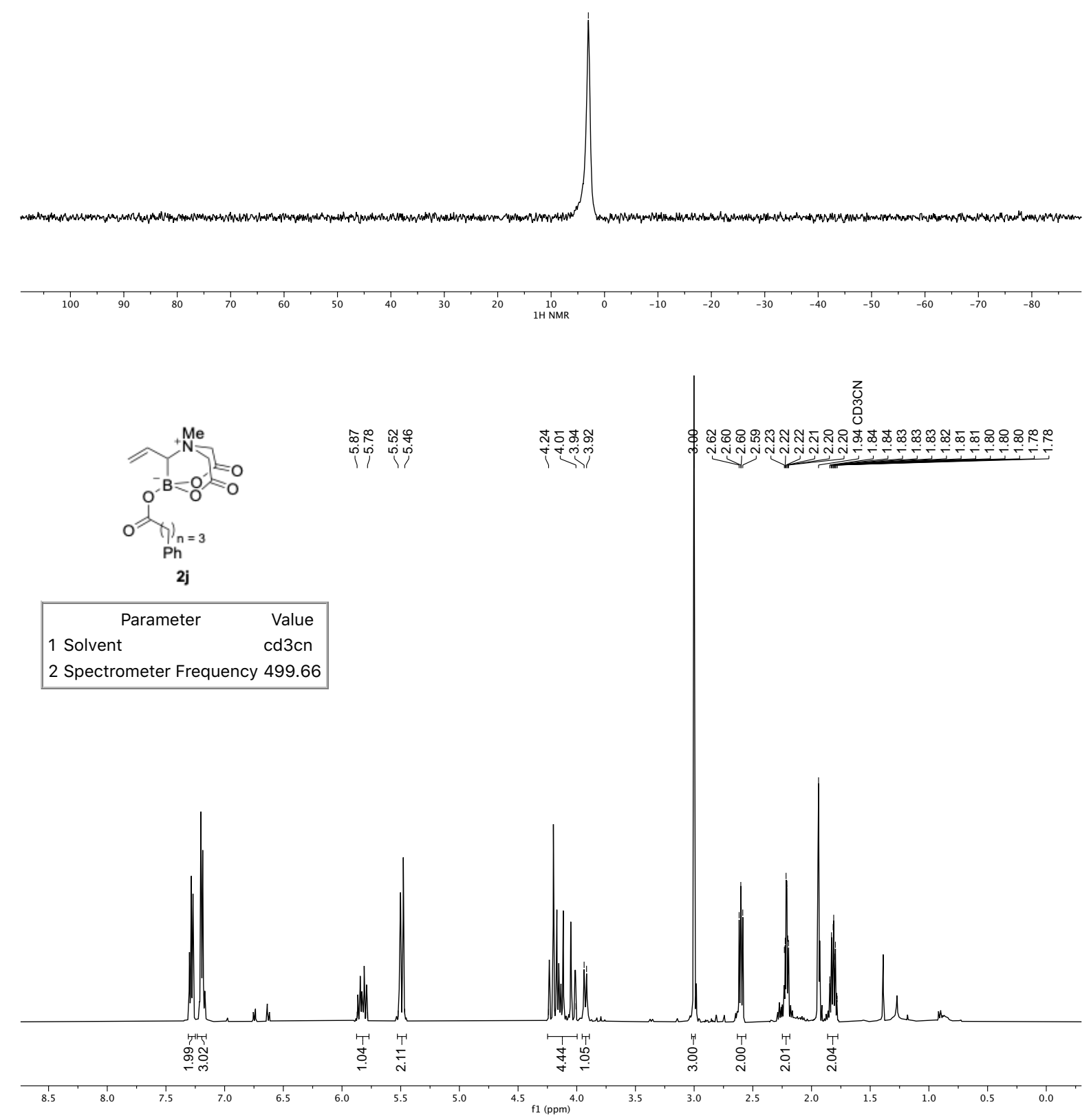

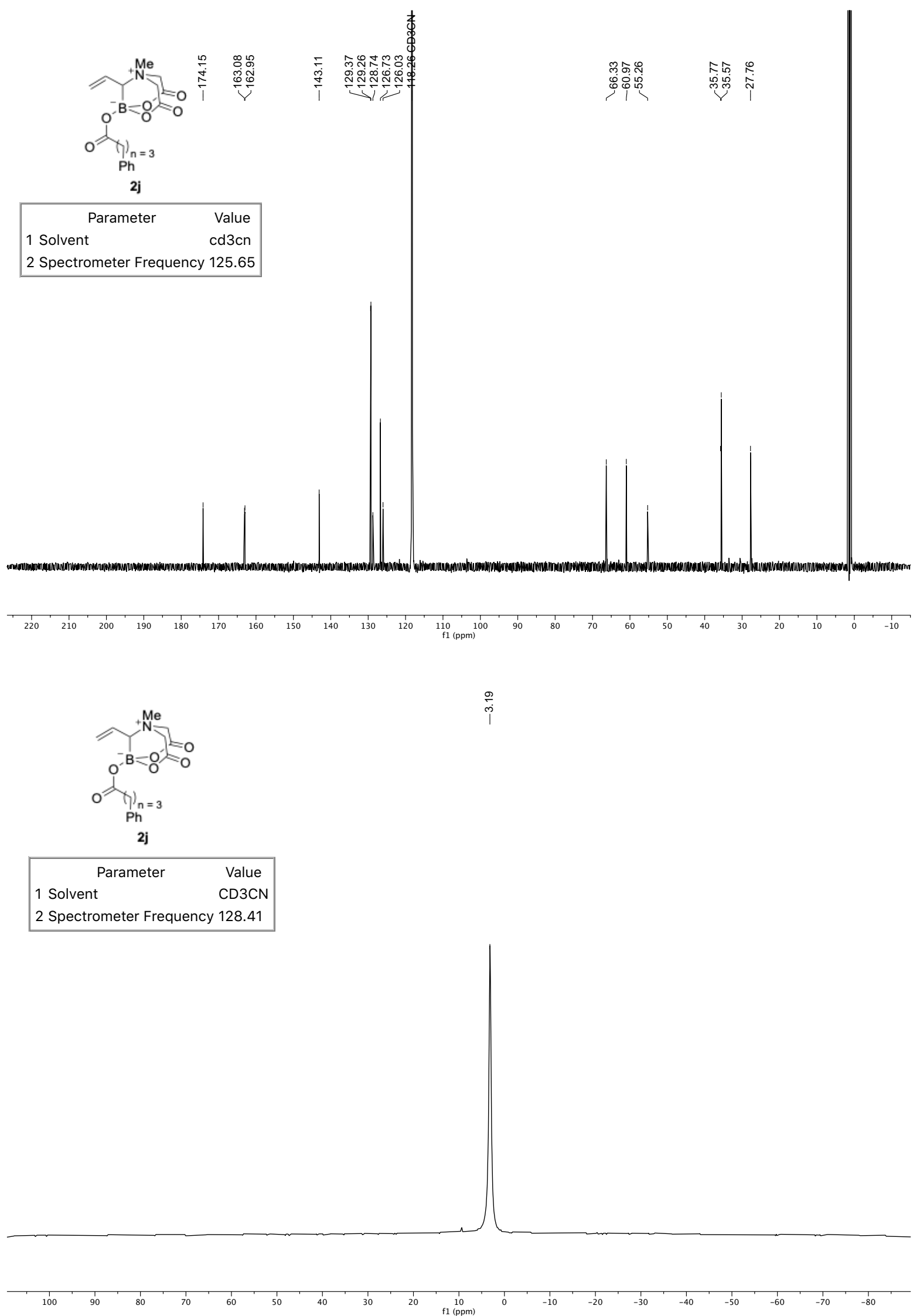


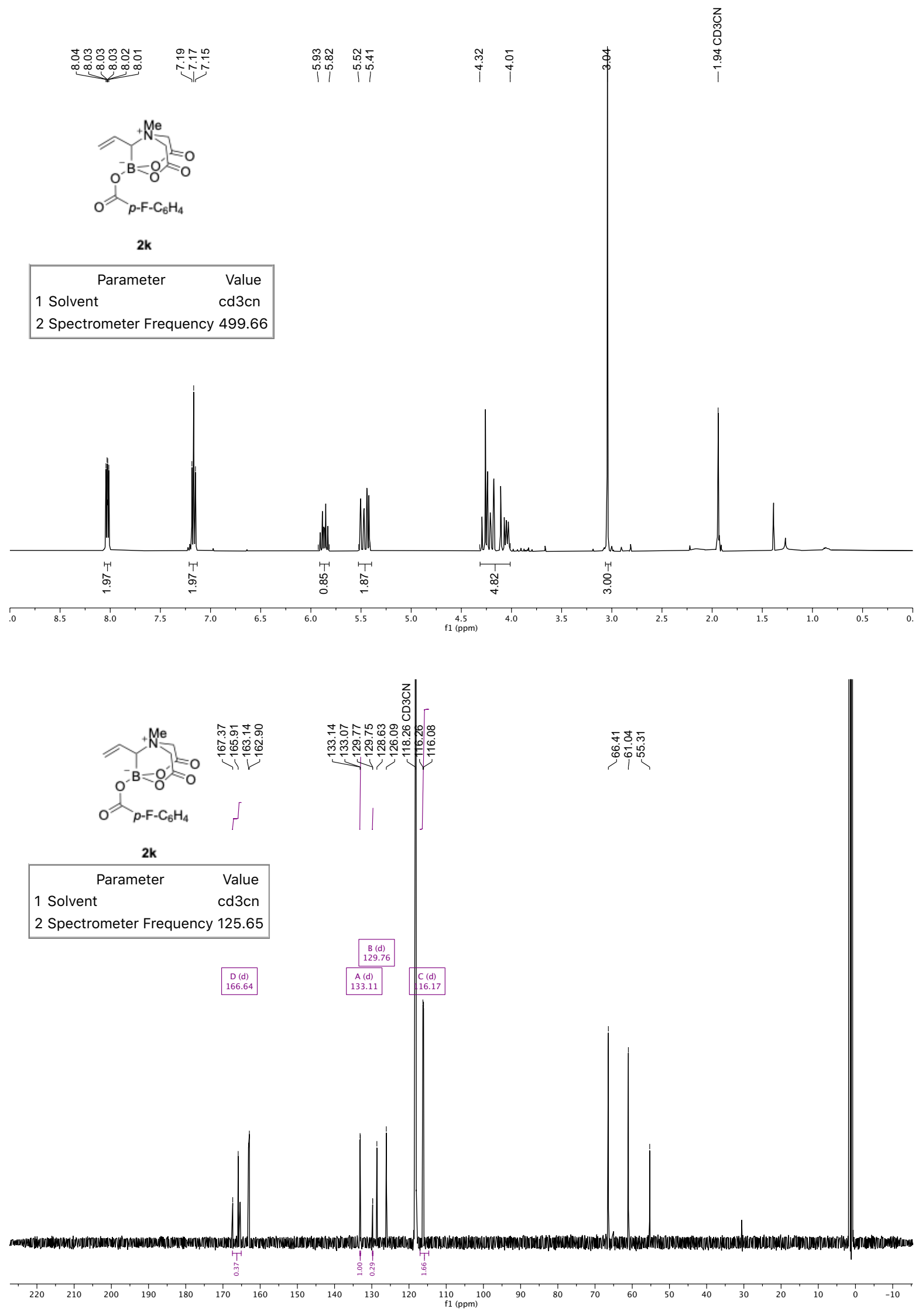




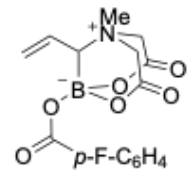

$2 k$

\begin{tabular}{|cc|}
\hline \multicolumn{1}{|c|}{ Parameter } & Value \\
1 Solvent & $\mathrm{CD} 3 \mathrm{CN}$ \\
2 Spectrometer Frequency & 128.41 \\
\hline
\end{tabular}

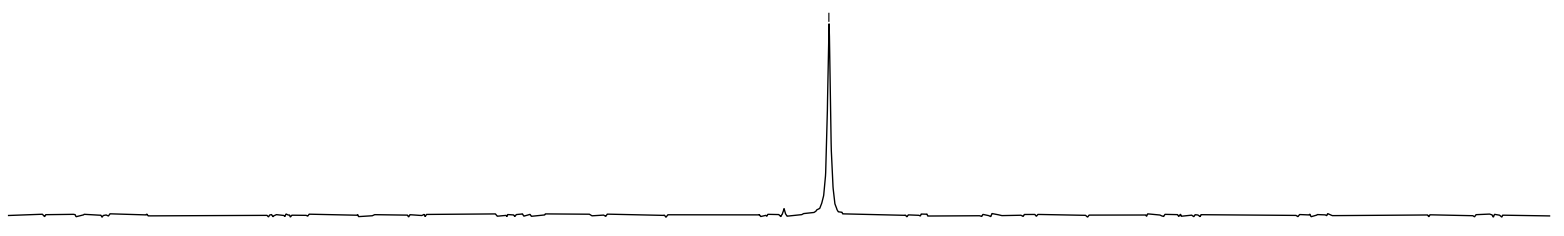

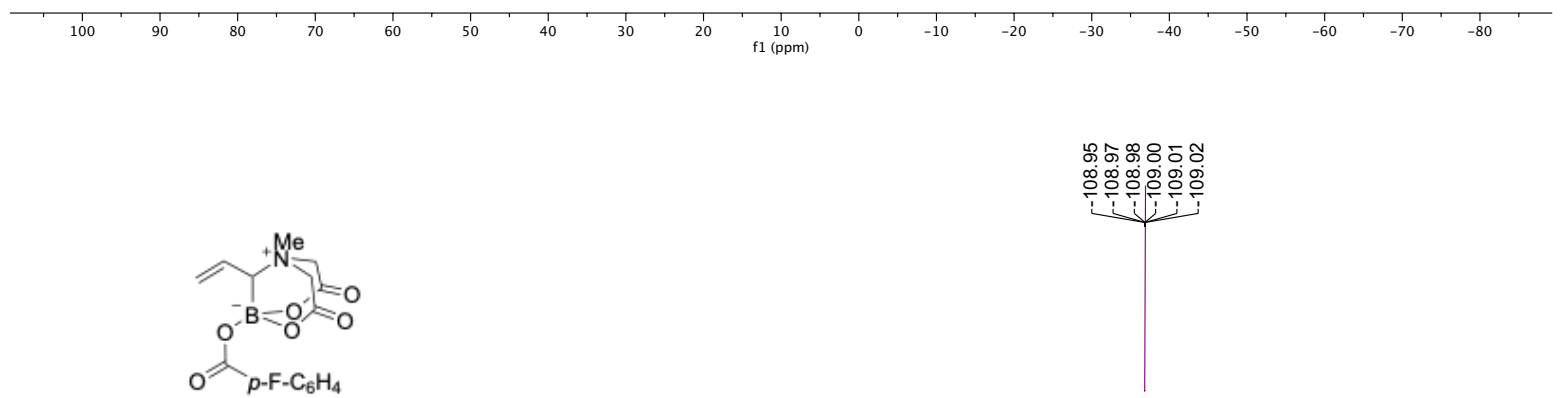

2k

\begin{tabular}{|lr|}
\hline \multicolumn{1}{|c|}{ Parameter } & \multicolumn{1}{c|}{ Value } \\
1 Solvent & CD3CN \\
2 Spectrometer Frequency & 376.58 \\
\hline
\end{tabular}

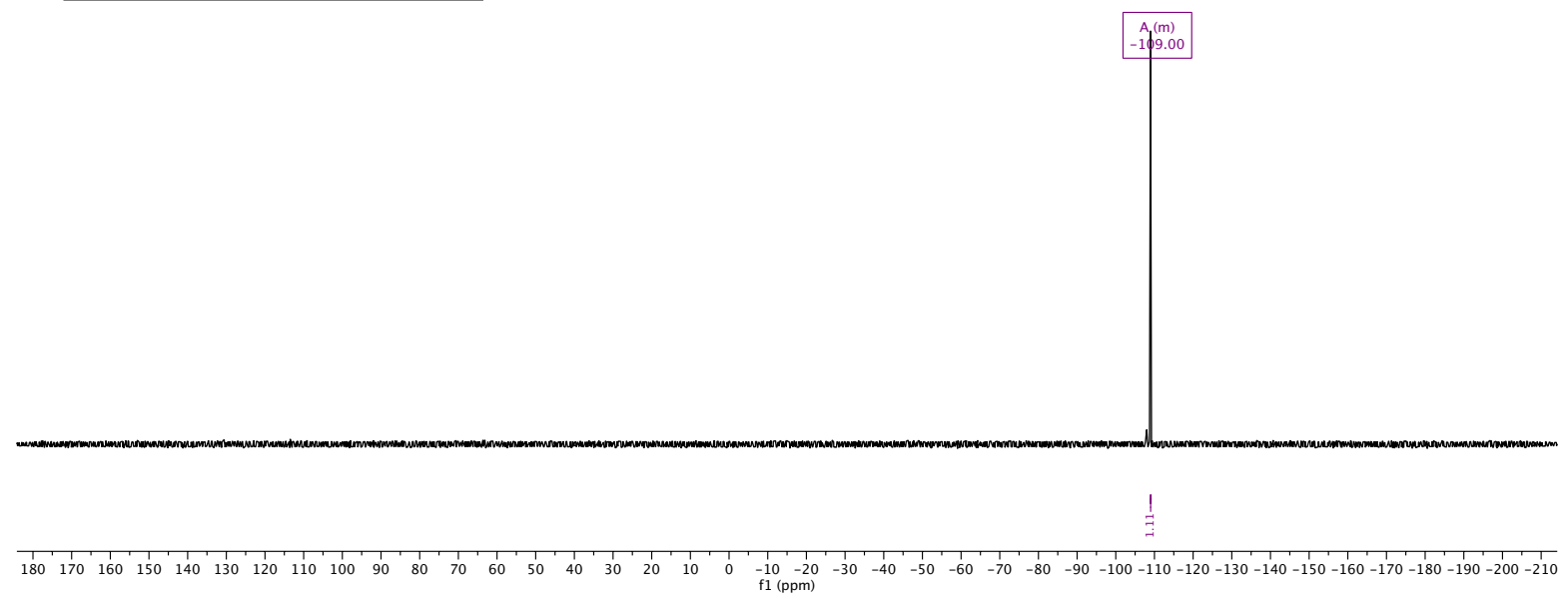



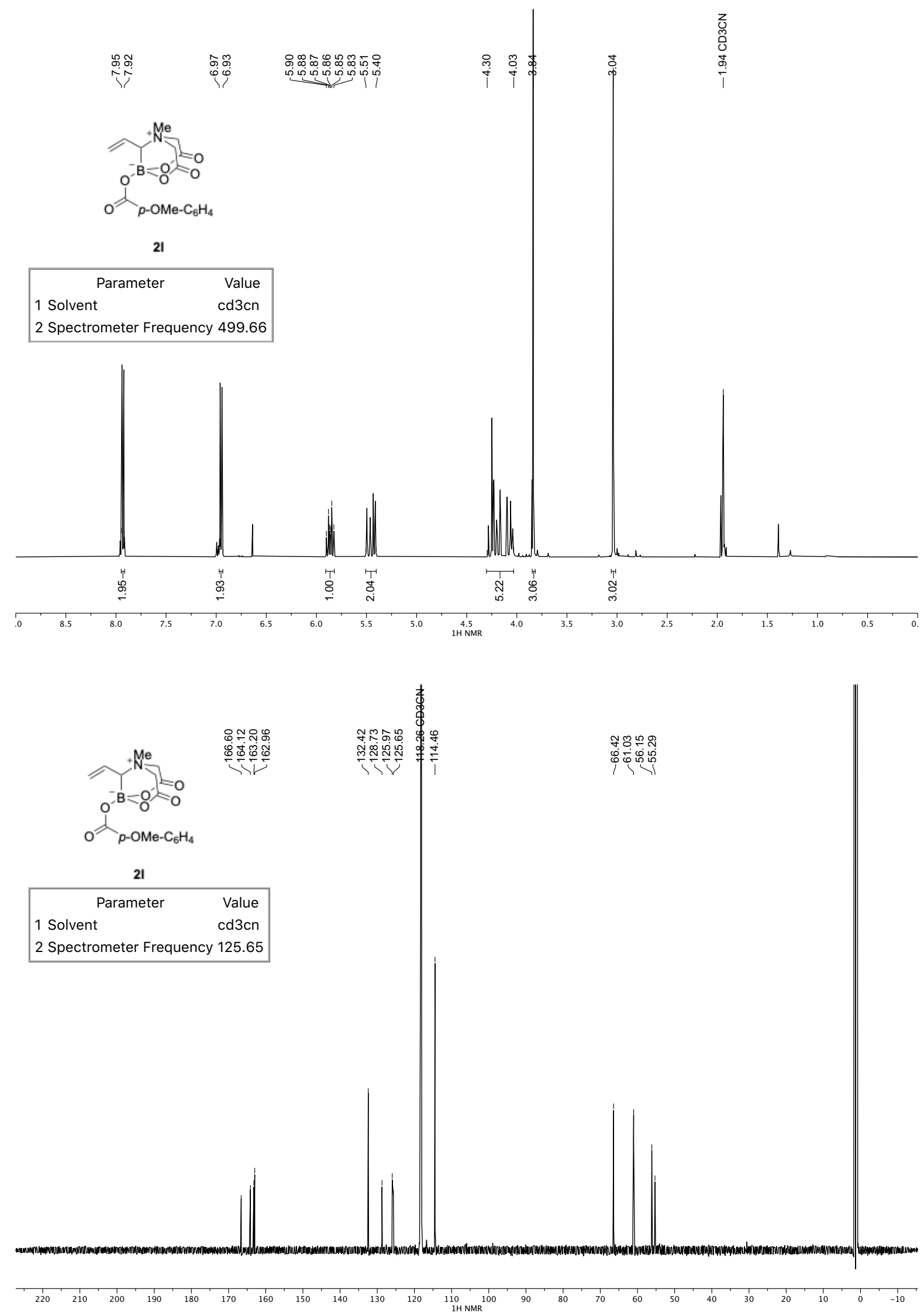


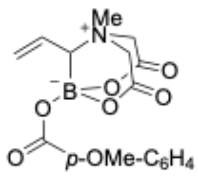

21

\begin{tabular}{|lc|}
\hline \multicolumn{1}{|c|}{ Parameter } & \multicolumn{1}{c|}{ Value } \\
1 Solvent & $\mathrm{CD} 3 \mathrm{CN}$ \\
2 Spectrometer Frequency & 128.41 \\
\hline
\end{tabular}
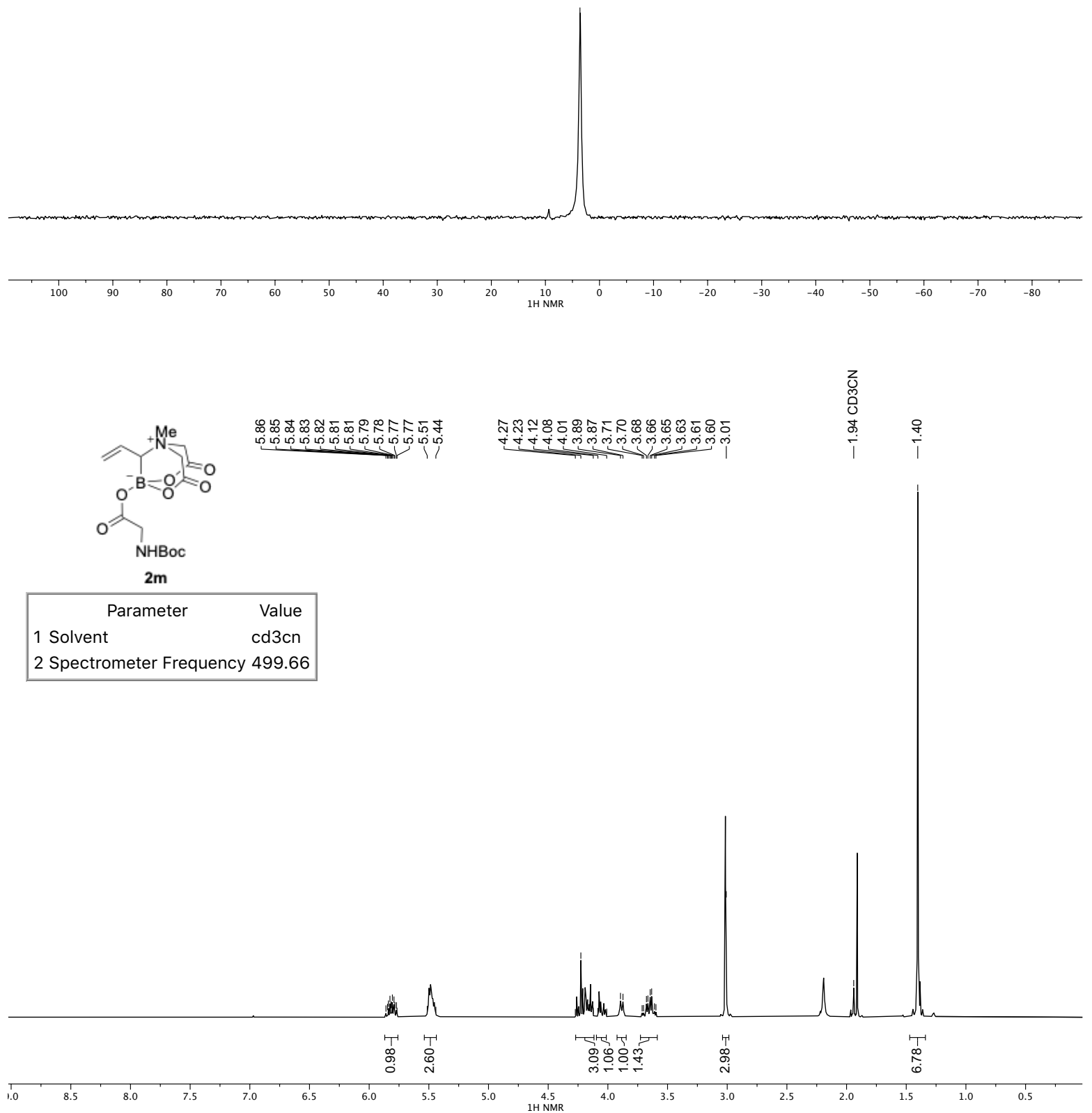

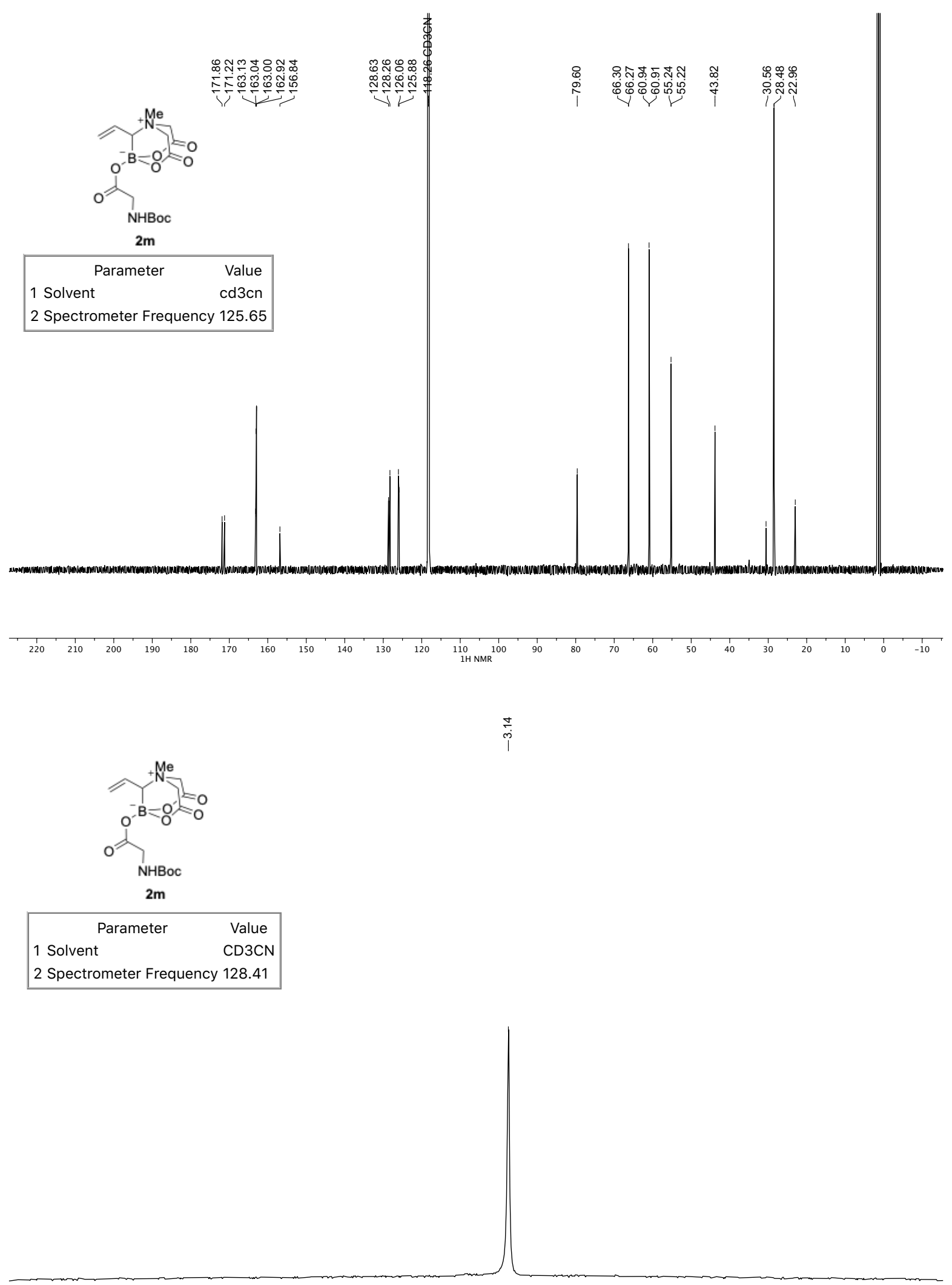

\begin{tabular}{|c|c|c|c|c|c|c|c|c|c|c|c|c|c|c|c|c|c|}
\hline 100 & 90 & 80 & 70 & 60 & 50 & 40 & 30 & 20 & 10 & 0 & -10 & -20 & -30 & -40 & -50 & $\begin{array}{l}1 \\
-60\end{array}$ & -70 \\
\hline
\end{tabular}



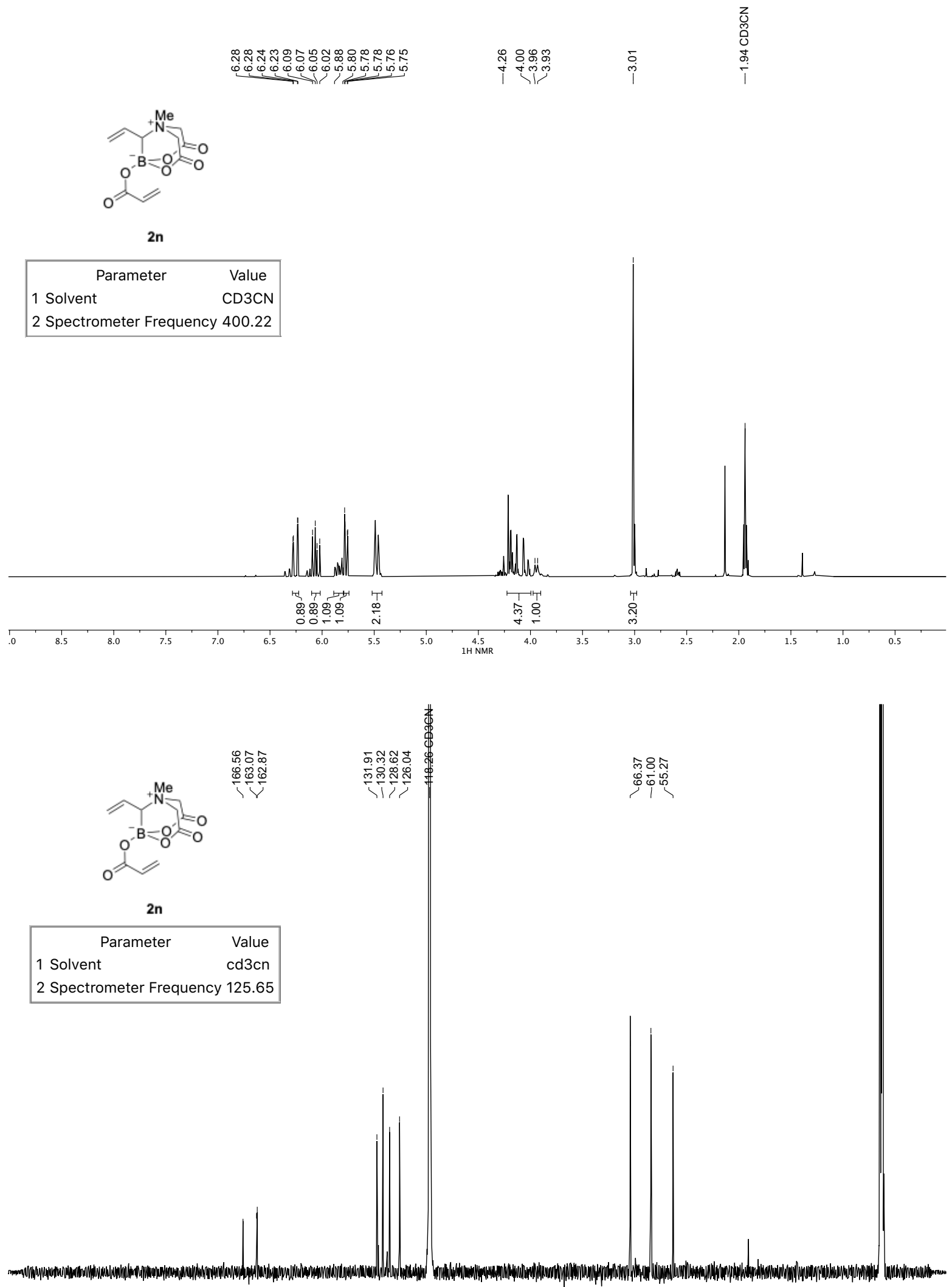

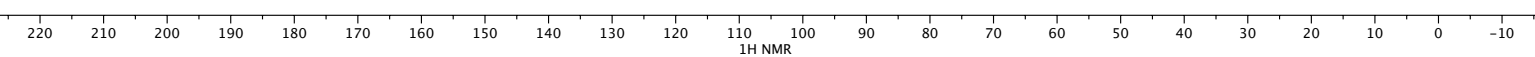




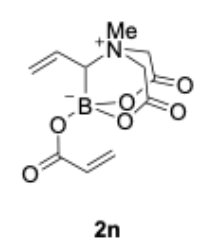

\begin{tabular}{|lc|}
\hline \multicolumn{1}{|c|}{ Parameter } & Value \\
1 Solvent & $\mathrm{CD} 3 \mathrm{CN}$ \\
2 Spectrometer Frequency & 128.41 \\
\hline
\end{tabular}
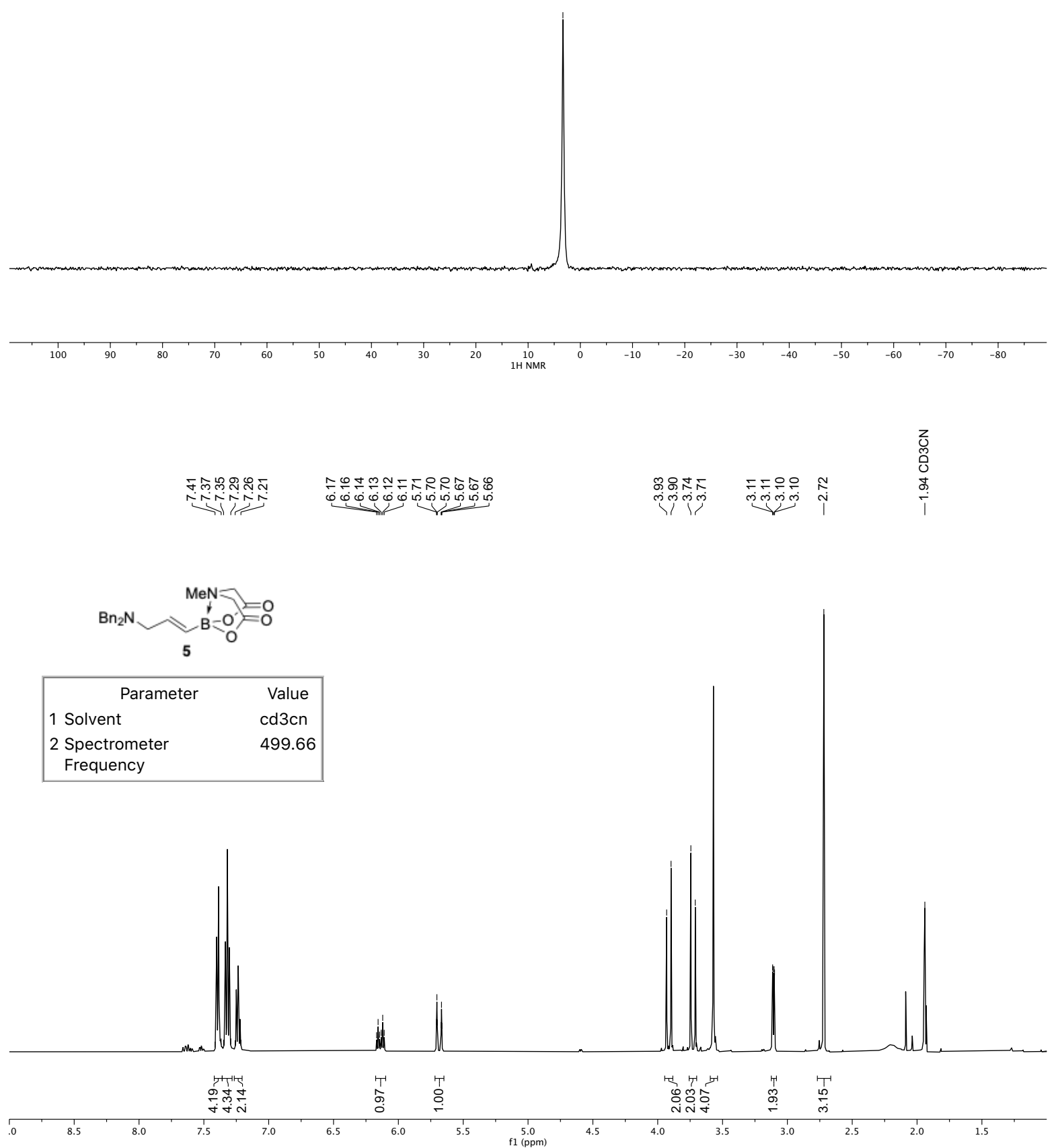


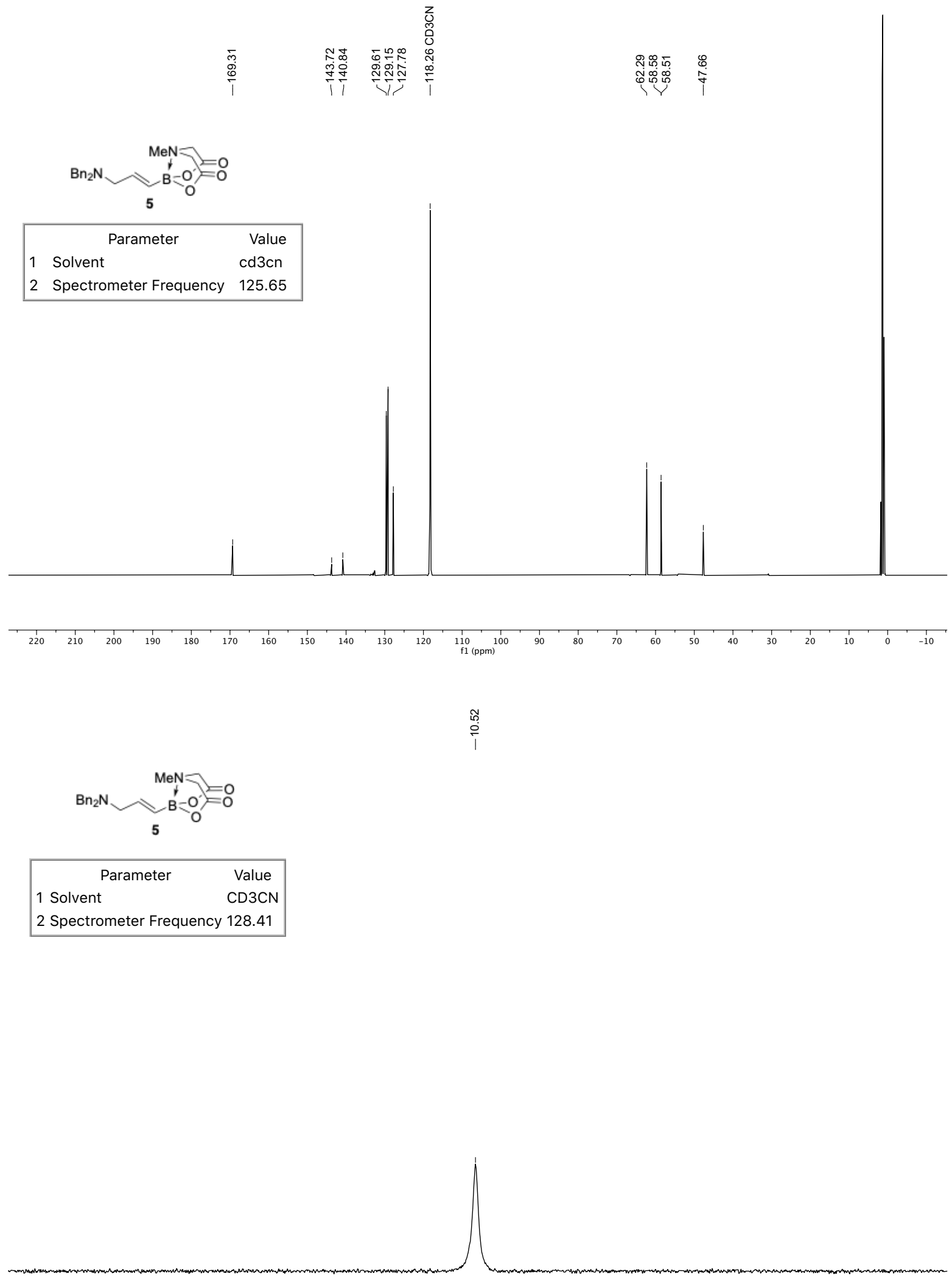

\begin{tabular}{|c|c|c|c|c|c|c|c|c|c|c|c|c|c|c|c|c|c|}
\hline 100 & 90 & 80 & 70 & 60 & 50 & 40 & 30 & 20 & 10 & 0 & -10 & -20 & $\begin{array}{l}1 \\
-30\end{array}$ & $\begin{array}{l}1 \\
-40\end{array}$ & -50 & $\begin{array}{l}1 \\
-60\end{array}$ & -70 \\
\hline
\end{tabular}




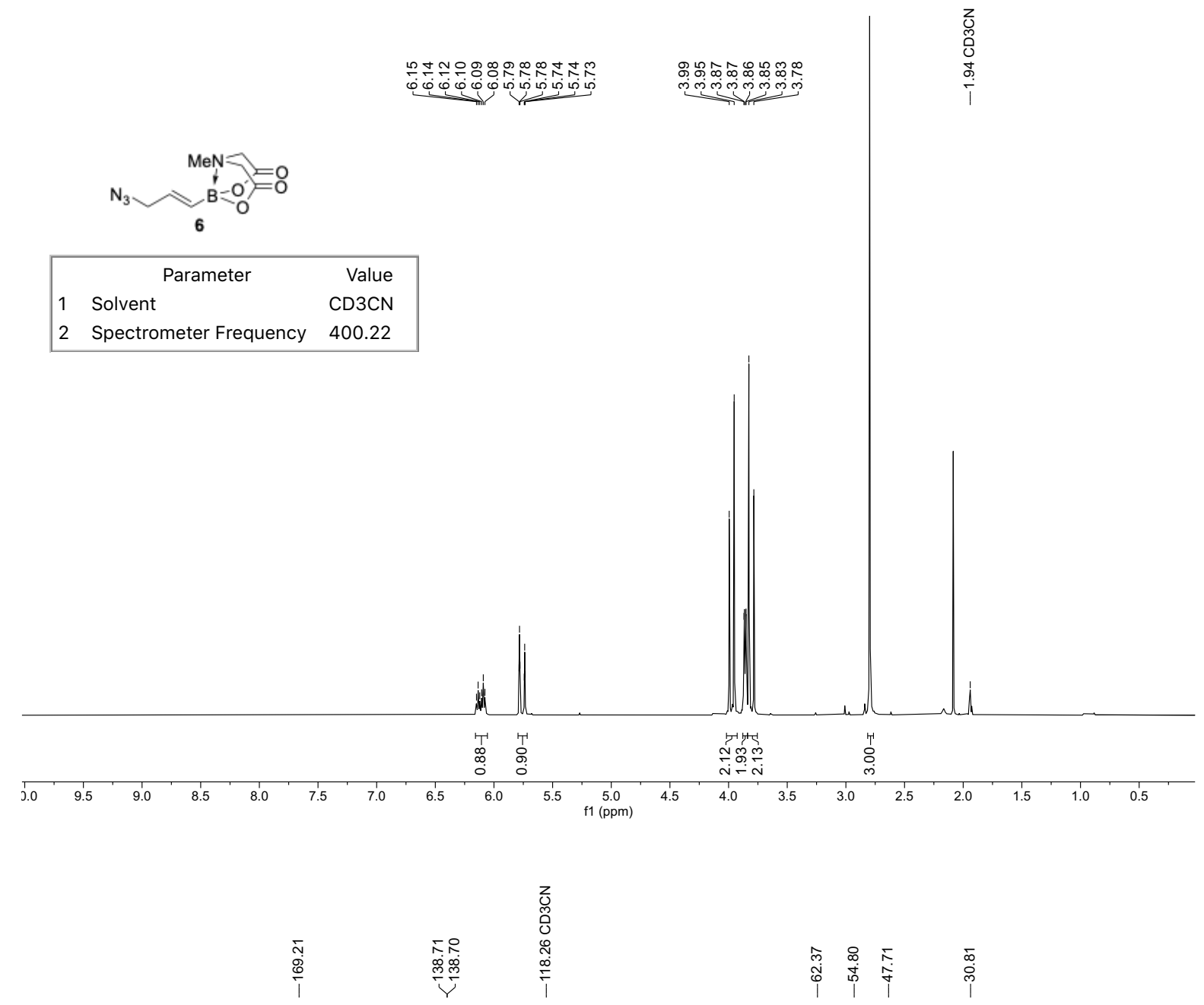

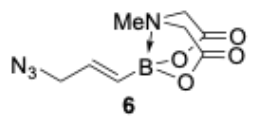

Parameter Value

1 Solvent

2 Spectrometer Frequency 125.65
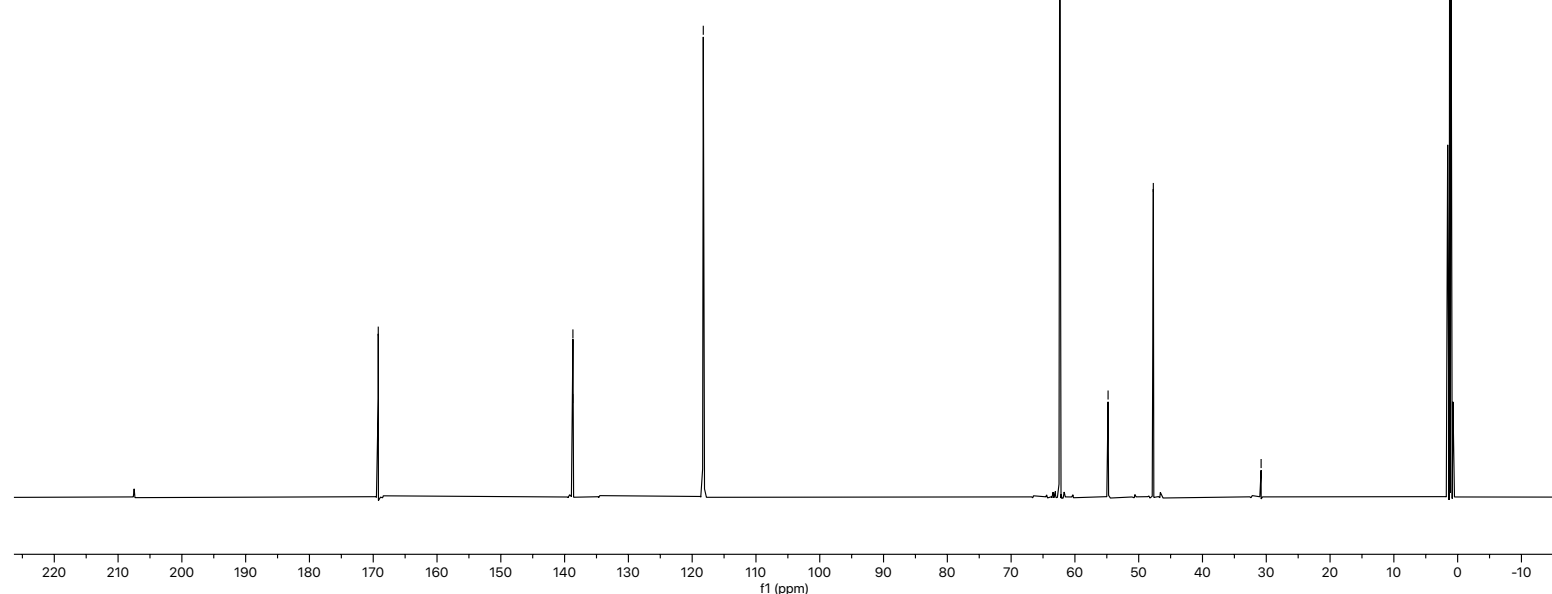


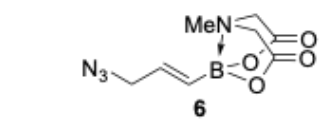

\begin{tabular}{|lc|}
\hline \multicolumn{1}{|c|}{ Parameter } & \multicolumn{1}{c|}{ Value } \\
1 Solvent & CD $3 \mathrm{CN}$ \\
2 Spectrometer Frequency & 128.41 \\
\hline
\end{tabular}
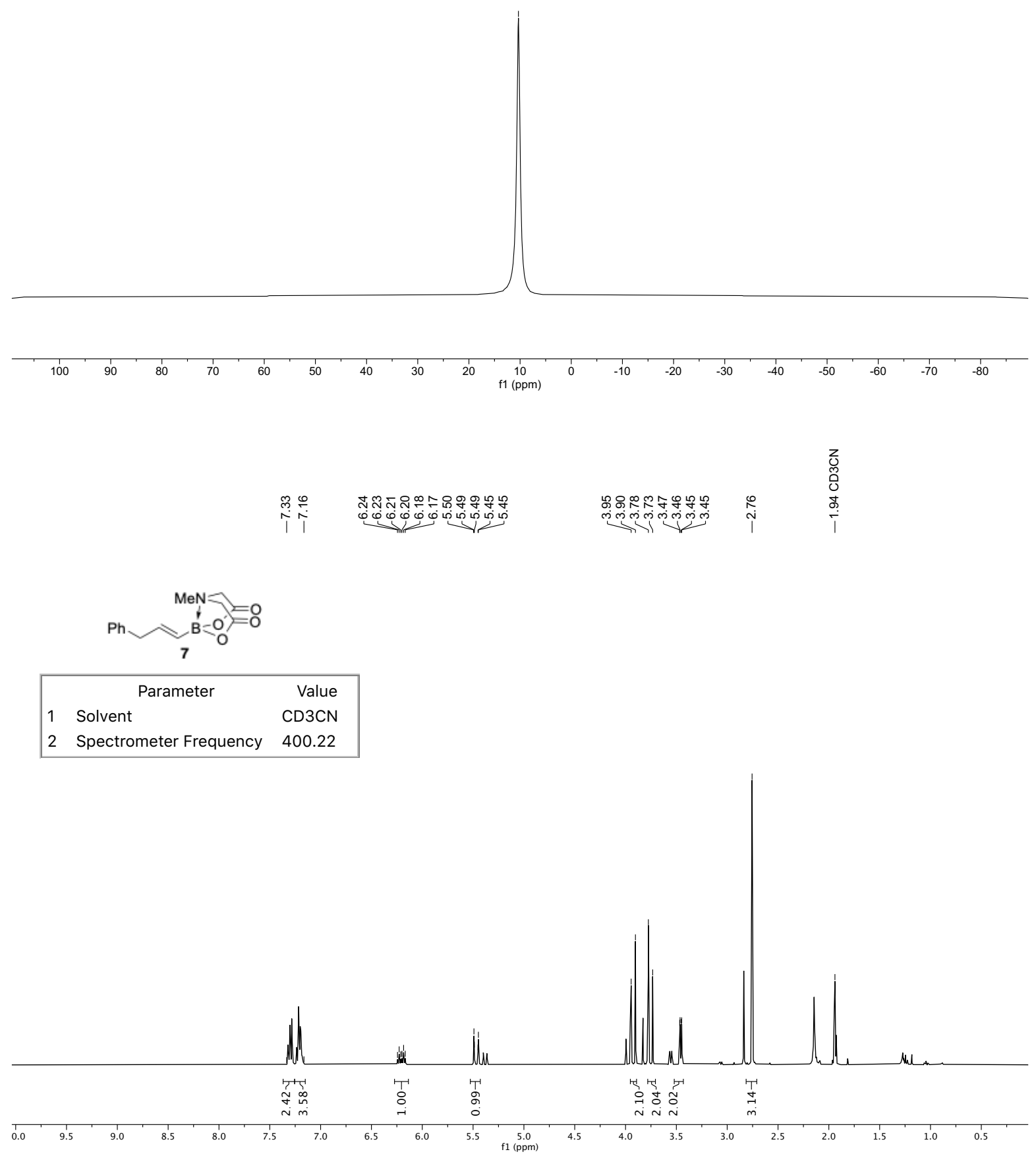

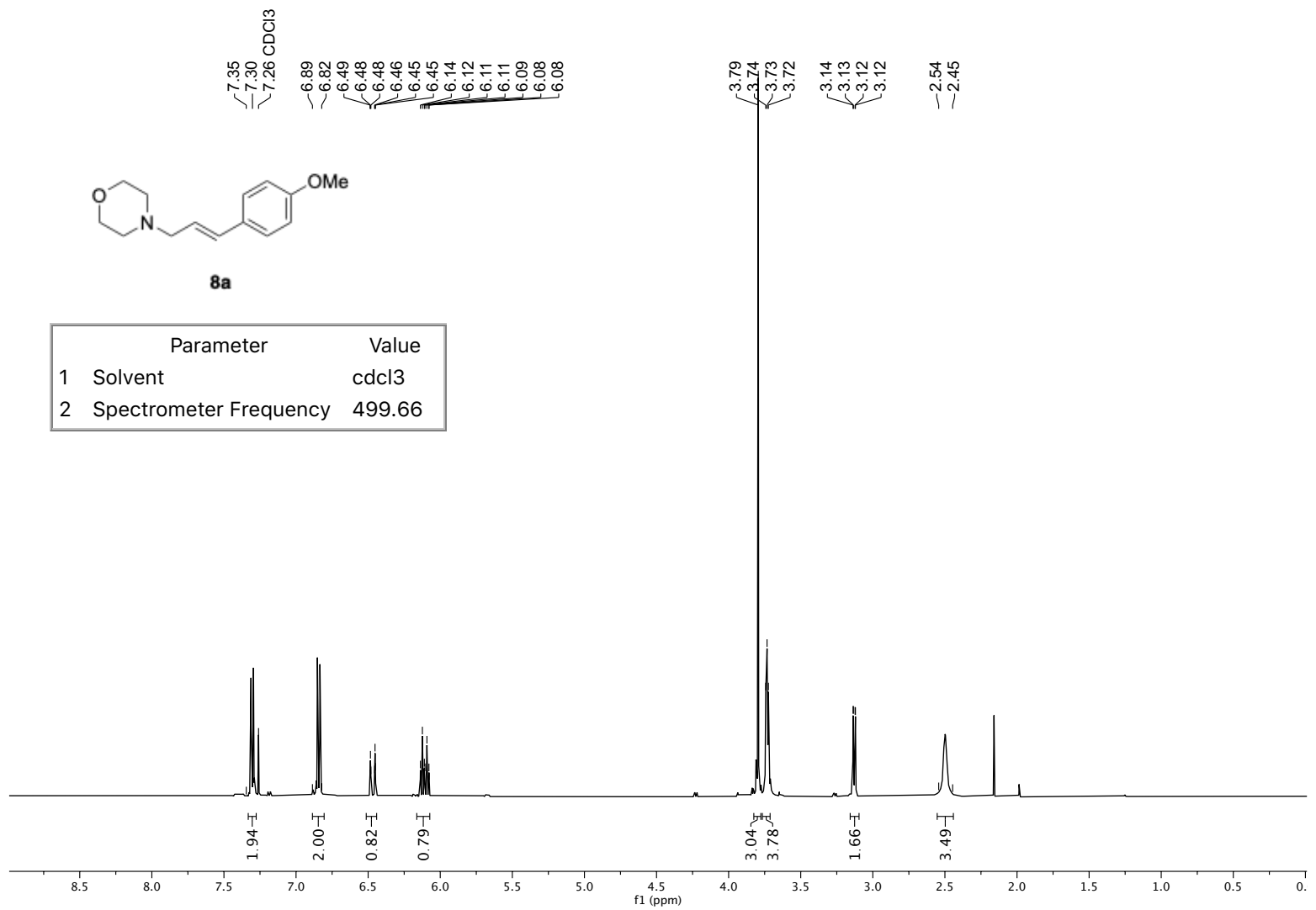

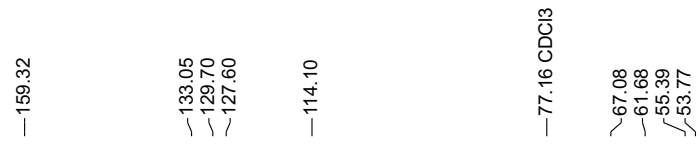

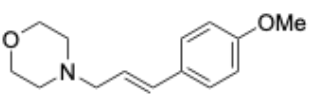

$8 a$

\begin{tabular}{lll} 
& \multicolumn{1}{c}{ Parameter } & \multicolumn{1}{c}{ Value } \\
1 & Solvent & cdcl3 \\
2 & Spectrometer Frequency & 125.65
\end{tabular}

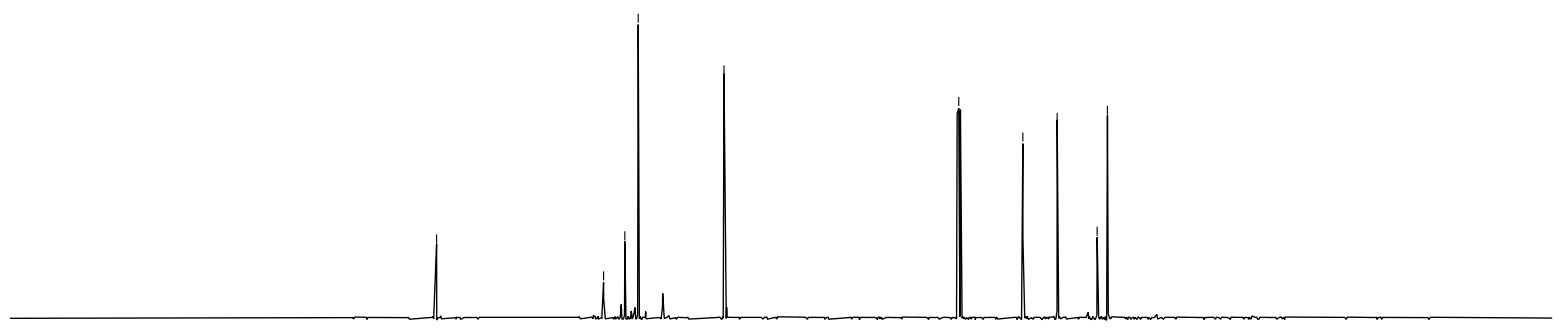

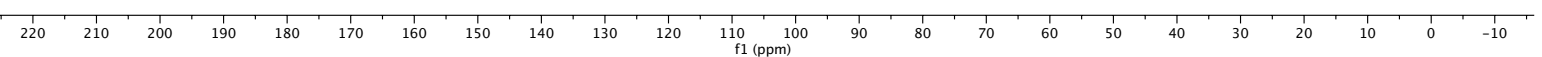



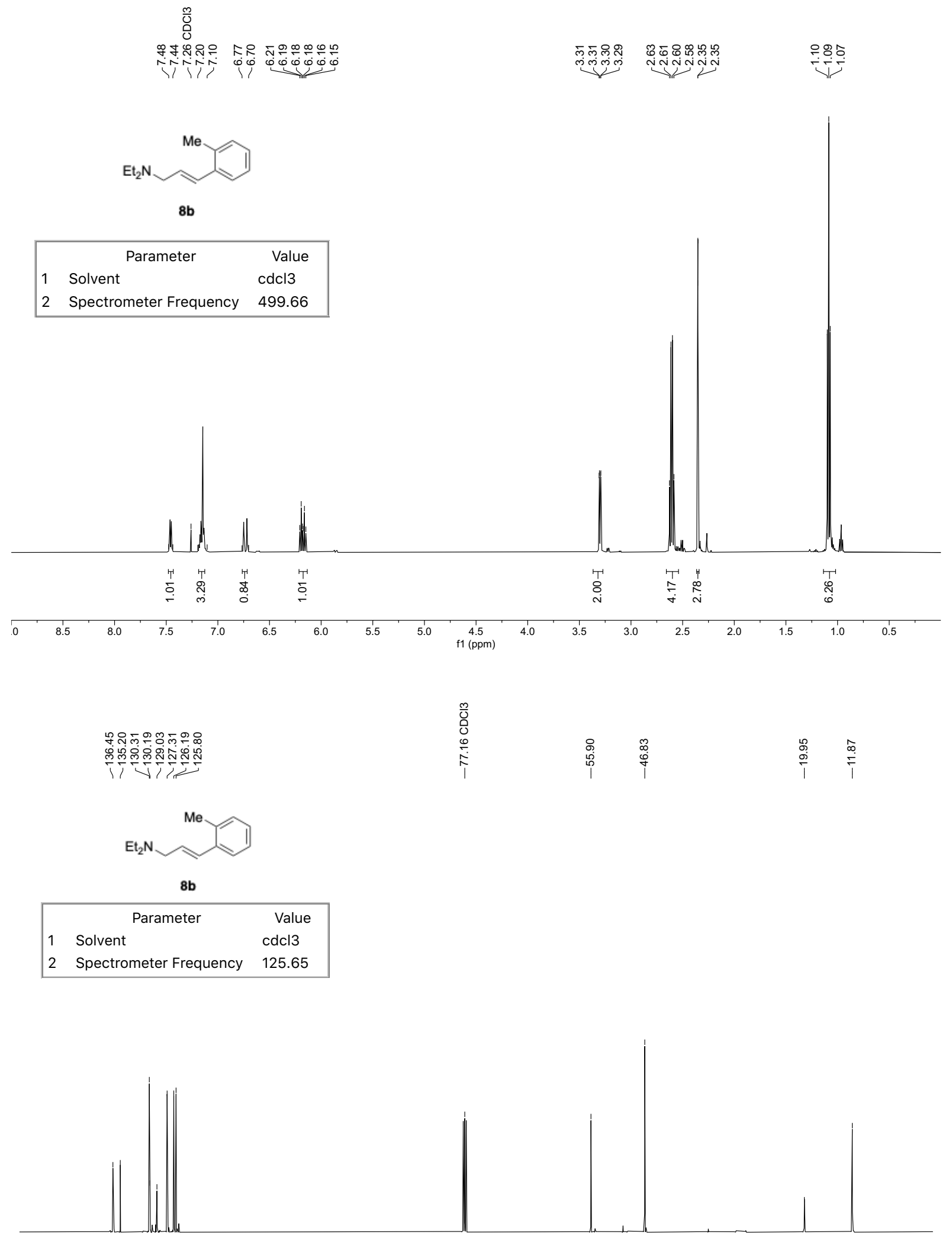

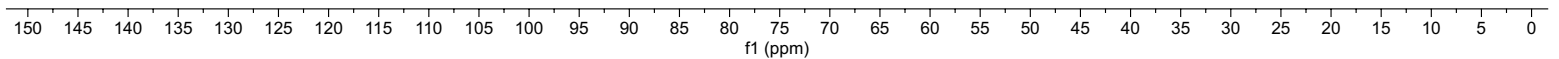



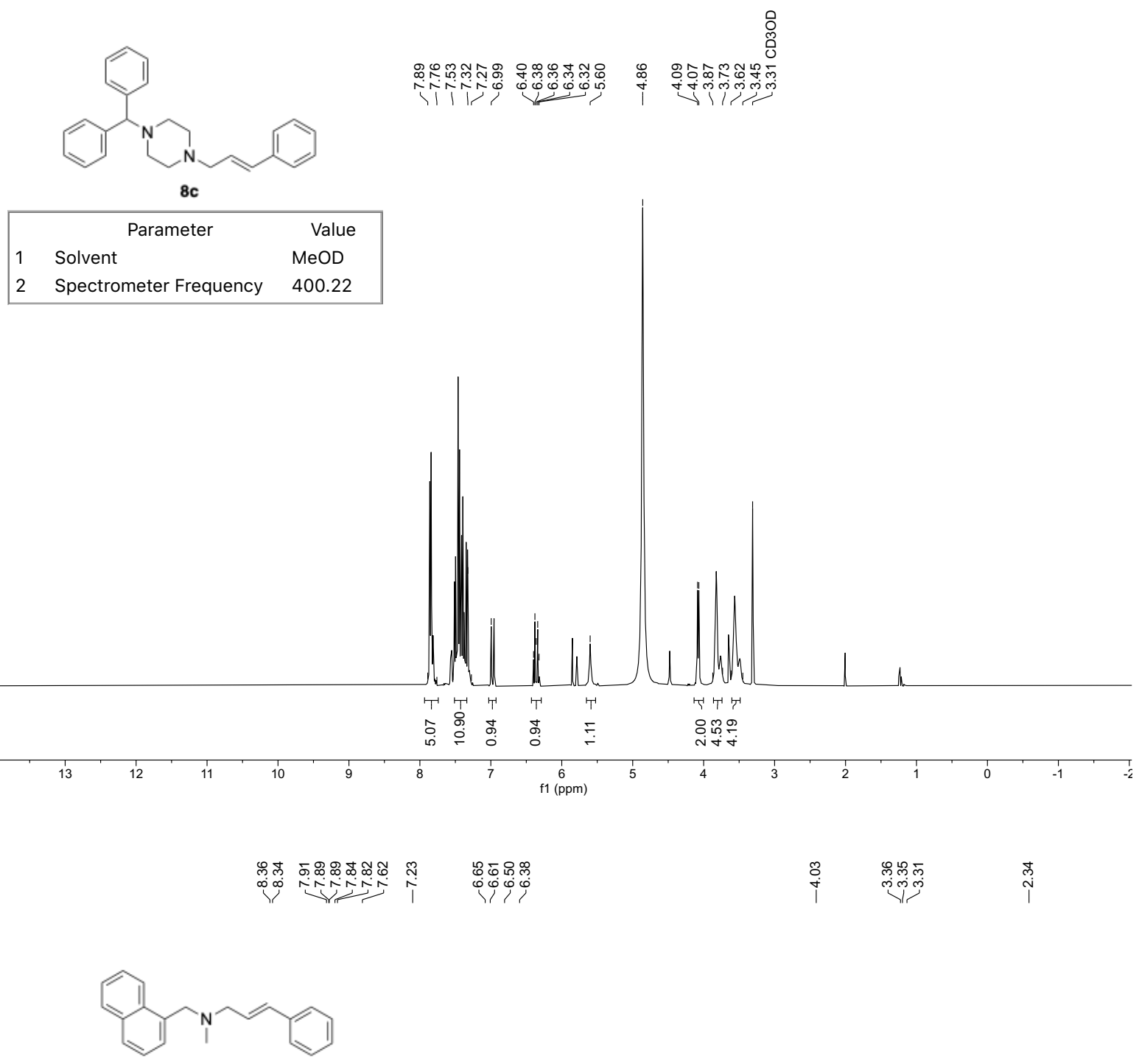

!
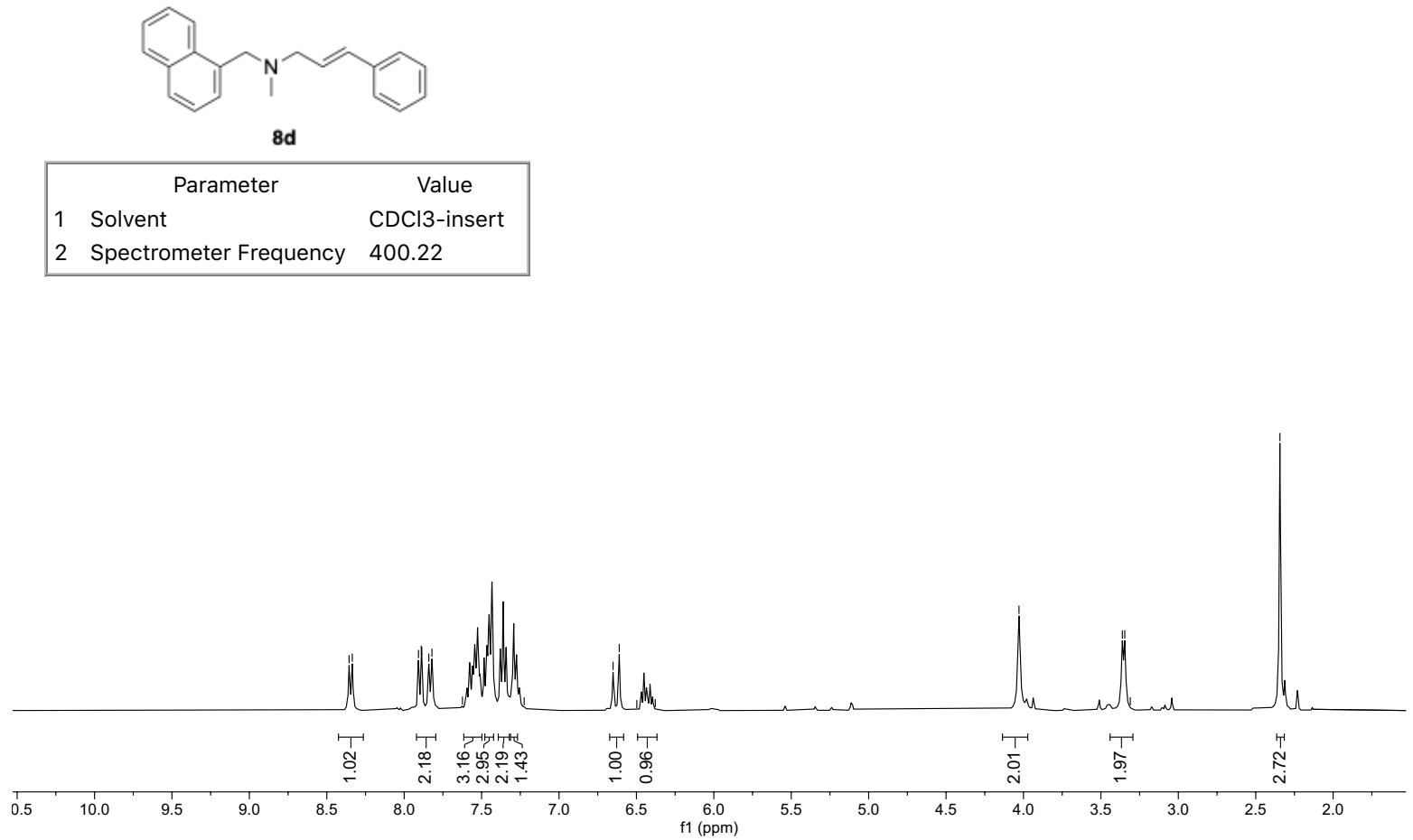


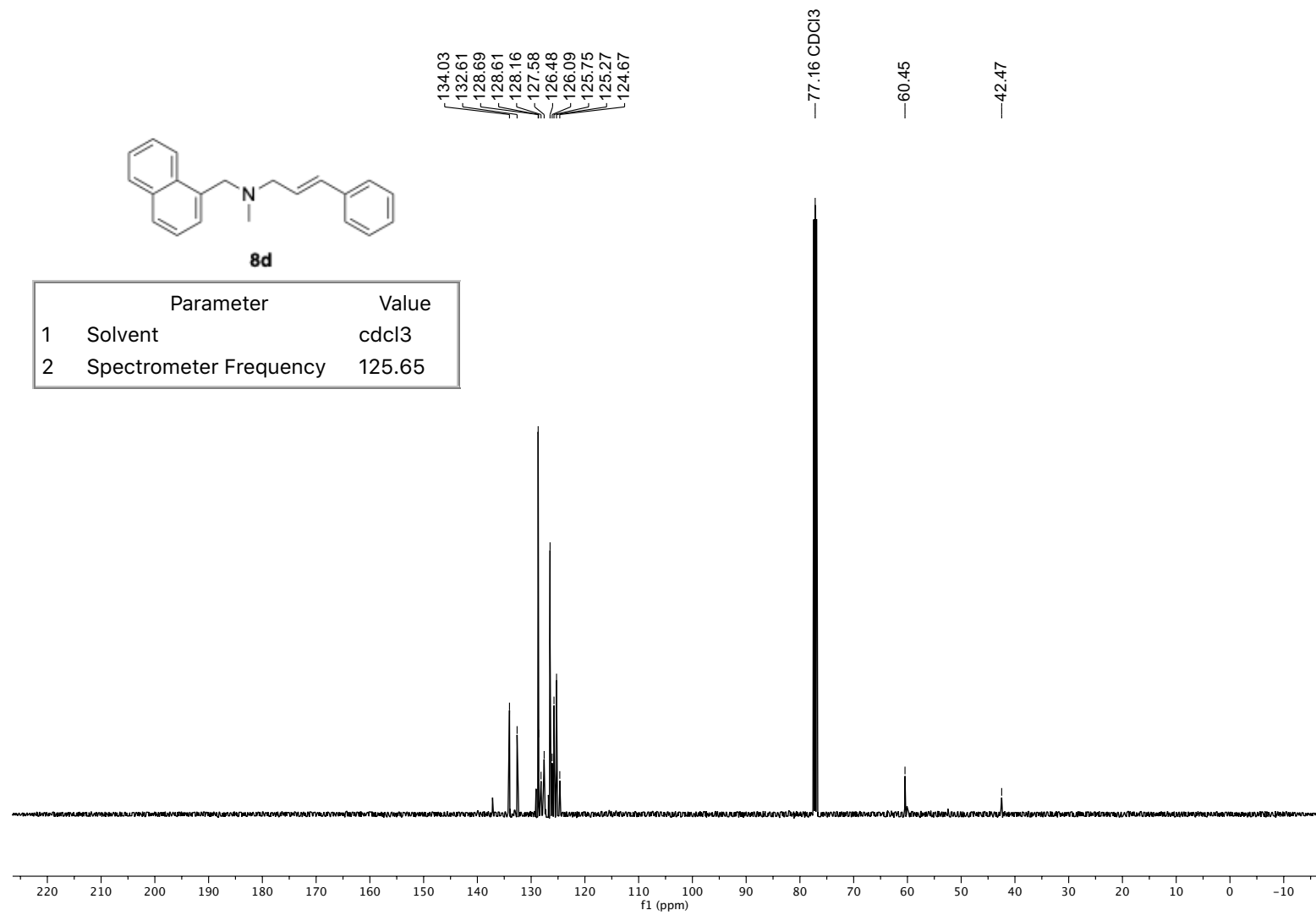




\section{$\underline{\text { X-Ray Data }}$}

\section{Compound 2c}

Compound $\mathbf{2 c}$ was recrystallized utilizing vapor diffusion. In a $4 \mathrm{ml}$ vial was dissolvent $20 \mathrm{mg}$ of $2 \mathbf{c}$ in $1 \mathrm{ml}$ of anhydrous acetone. The vial was then placed in a $20 \mathrm{ml}$ vial containing pentane. The larger vial was capped and placed in the dark for 4 days before X-ray quality crystals were observed.

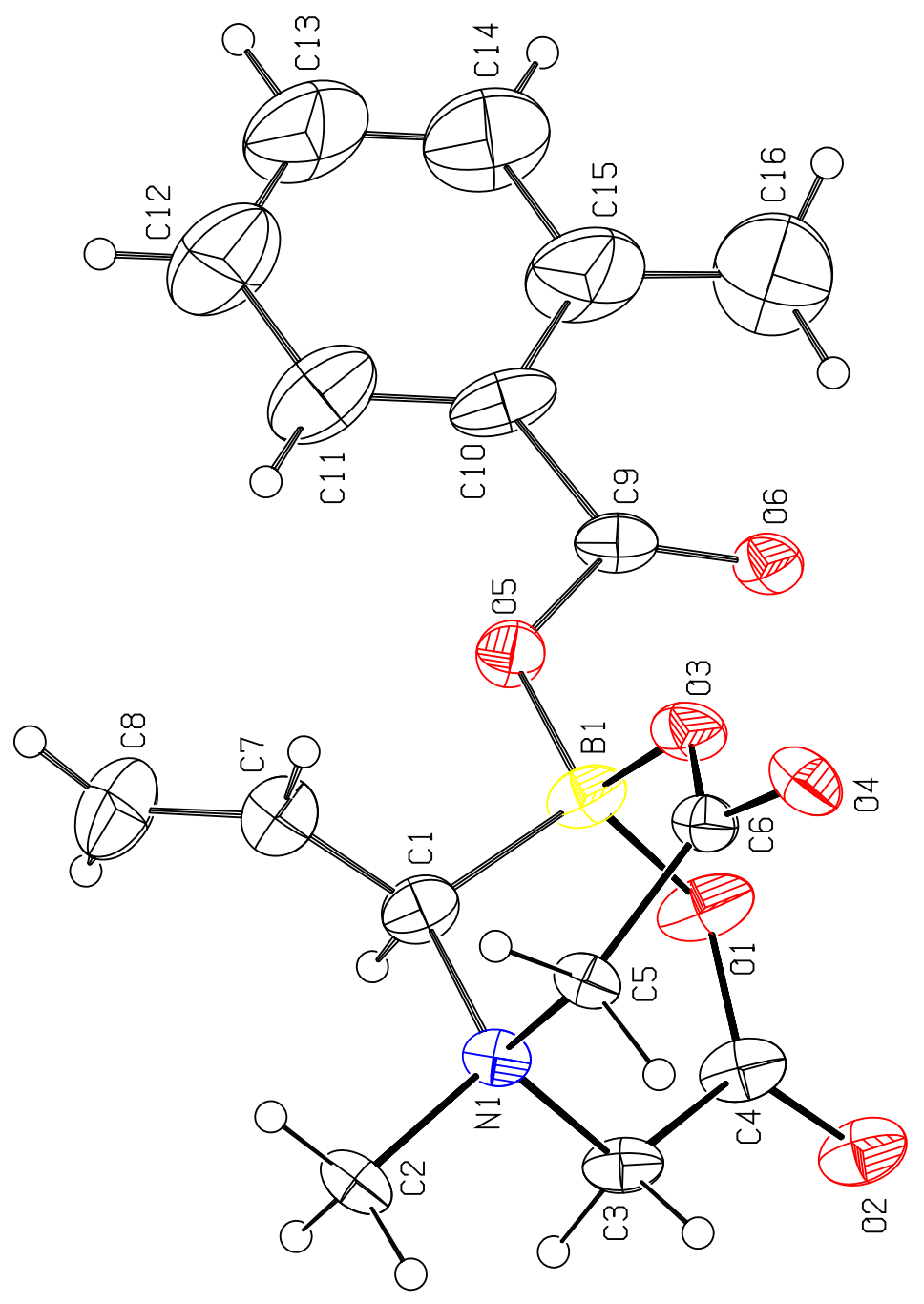

Figure S-4. X-ray crystal structure of compound $\mathbf{2 c}$ with 50\% thermal ellipsoid probabilities.

Table S-3. Crystal data and structure refinement for $\mathbf{2 c}$.

\begin{tabular}{|l|l|}
\hline Identification code & 2c \\
\hline Empirical formula & $\mathrm{C}_{16} \mathrm{H}_{18} \mathrm{BNO}_{6}$ \\
\hline Formula weight & 331.12 \\
\hline Temperature & $150(2) \mathrm{K}$ \\
\hline
\end{tabular}




\begin{tabular}{|l|l|}
\hline Wavelength & $1.54178 \AA$ \\
\hline Crystal system & Monoclinic \\
\hline Space group & $\mathrm{P} 2 \mathrm{c}$ \\
\hline Unit cell dimensions & $\mathrm{a}=19.1751(9) \AA \quad \mathrm{a}=90^{\circ}$. \\
& $\mathrm{b}=6.6256(3) \AA \quad \mathrm{b}=104.204(4)^{\circ}$. \\
& $\mathrm{c}=13.0047(6) \AA \quad \mathrm{g}=90^{\circ}$. \\
\hline Volume & $1601.69(13) \AA^{3}$ \\
\hline$Z$ & 4 \\
\hline Density (calculated) & $1.373 \mathrm{Mg} / \mathrm{m}^{3}$ \\
\hline Absorption coefficient & $0.871 \mathrm{~mm}^{-1}$ \\
\hline F(000) & 696 \\
\hline Crystal size & $0.180 \mathrm{x} 0.180 \mathrm{x} 0.010 \mathrm{~mm}^{3}$ \\
\hline Theta range for data collection & 2.377 to $67.711^{\circ}$. \\
\hline Index ranges & $-23<=\mathrm{h}<=22,-7<=\mathrm{k}<=7,-15<=1<=15$ \\
\hline Reflections collected & 28528 \\
\hline Independent reflections & $2888[\mathrm{R}($ int $)=0.0733]$ \\
\hline Completeness to theta $=67.679^{\circ}$ & $99.8 \%$ \\
\hline Absorption correction & Semi-empirical from equivalents \\
\hline Max. and min. transmission & 0.7530 and 0.6316 \\
\hline Refinement method & Full-matrix least-squares on $\mathrm{F}^{2}$ \\
\hline Data / restraints / parameters & $2888 / 331 / 302$ \\
\hline Goodness-of-fit on F 2 & 1.069 \\
\hline Final R indices [I $>2$ sigma(I)] & $\mathrm{R} 1=0.0788, \mathrm{wR} 2=0.2282$ \\
\hline R indices (all data) & $\mathrm{R} 1=0.0974, \mathrm{wR} 2=0.2488$ \\
\hline Extinction coefficient & $\mathrm{n} / \mathrm{a}$ \\
\hline
\end{tabular}

Table S-4. Atomic coordinates (x $\left.10^{4}\right)$ and equivalent isotropic displacement parameters $\left(\AA^{2} \mathrm{x}\right.$ $10^{3}$ ) for 2 c. $\mathrm{U}(\mathrm{eq})$ is defined as one third of the trace of the orthogonalized Uij tensor.

\begin{tabular}{|l|l|l|l|l|}
\hline Atom & $\mathbf{x}$ & $\mathbf{y}$ & $\mathbf{z}$ & $\mathbf{U}(\mathbf{e q})$ \\
\hline $\mathrm{C}(2)$ & $8750(2)$ & $-879(5)$ & $2220(2)$ & $47(1)$ \\
\hline $\mathrm{O}(1)$ & $8668(1)$ & $4790(3)$ & $3770(2)$ & $60(1)$ \\
\hline $\mathrm{O}(2)$ & $9765(1)$ & $5395(3)$ & $3596(2)$ & $54(1)$ \\
\hline $\mathrm{O}(3)$ & $8400(1)$ & $2271(3)$ & $4943(2)$ & $47(1)$ \\
\hline $\mathrm{O}(4)$ & $9132(1)$ & $67(4)$ & $5952(2)$ & $51(1)$ \\
\hline $\mathrm{N}(1)$ & $8752(1)$ & $665(3)$ & $3076(2)$ & $35(1)$ \\
\hline $\mathrm{C}(3)$ & $9302(2)$ & $2230(4)$ & $2996(3)$ & $41(1)$ \\
\hline $\mathrm{C}(4)$ & $9264(2)$ & $4251(5)$ & $3512(3)$ & $47(1)$ \\
\hline $\mathrm{C}(5)$ & $8960(2)$ & $-410(4)$ & $4125(2)$ & $32(1)$ \\
\hline $\mathrm{C}(6)$ & $8841(2)$ & $714(4)$ & $5080(2)$ & $38(1)$ \\
\hline $\mathrm{C}(1)$ & $8017(2)$ & $1662(5)$ & $2950(3)$ & $50(1)$ \\
\hline $\mathrm{O}(5)$ & $7416(2)$ & $4201(8)$ & $3763(4)$ & $47(1)$ \\
\hline $\mathrm{O}(6)$ & $7798(2)$ & $6795(4)$ & $4883(3)$ & $42(1)$ \\
\hline $\mathrm{C}(7)$ & $7432(3)$ & $336(8)$ & $2839(5)$ & $57(1)$ \\
\hline $\mathrm{C}(8)$ & $6881(4)$ & $177(13)$ & $2000(6)$ & $88(2)$ \\
\hline
\end{tabular}




\begin{tabular}{|l|l|l|l|l|}
\hline $\mathrm{C}(9)$ & $7329(3)$ & $5814(10)$ & $4359(5)$ & $45(1)$ \\
\hline $\mathrm{C}(1 \mathrm{~A})$ & $8017(2)$ & $1662(5)$ & $2950(3)$ & $50(1)$ \\
\hline $\mathrm{O}(5 \mathrm{~A})$ & $7528(6)$ & $4174(18)$ & $4288(9)$ & $50(2)$ \\
\hline $\mathrm{O}(6 \mathrm{~A})$ & $7398(6)$ & $6550(20)$ & $3145(11)$ & $99(4)$ \\
\hline $\mathrm{C}(7 \mathrm{~A})$ & $7628(6)$ & $2136(18)$ & $1932(8)$ & $45(2)$ \\
\hline $\mathrm{C}(8 \mathrm{~A})$ & $6998(7)$ & $1560(30)$ & $1434(11)$ & $68(3)$ \\
\hline $\mathrm{C}(9 \mathrm{~A})$ & $7194(8)$ & $5680(30)$ & $3881(15)$ & $55(3)$ \\
\hline $\mathrm{C}(10)$ & $6541(3)$ & $6291(15)$ & $4129(9)$ & $76(2)$ \\
\hline $\mathrm{C}(11)$ & $6026(4)$ & $5130(12)$ & $3441(7)$ & $106(2)$ \\
\hline $\mathrm{C}(12)$ & $5301(3)$ & $5606(12)$ & $3274(7)$ & $128(3)$ \\
\hline $\mathrm{C}(13)$ & $5090(3)$ & $7244(13)$ & $3794(7)$ & $138(3)$ \\
\hline $\mathrm{C}(14)$ & $5605(4)$ & $8405(12)$ & $4481(7)$ & $139(3)$ \\
\hline $\mathrm{C}(15)$ & $6330(4)$ & $7929(15)$ & $4649(8)$ & $118(3)$ \\
\hline $\mathrm{C}(16)$ & $6820(9)$ & $9360(20)$ & $5340(12)$ & $141(5)$ \\
\hline $\mathrm{C}(10 \mathrm{~A})$ & $6510(8)$ & $6090(40)$ & $4220(20)$ & $92(3)$ \\
\hline $\mathrm{C}(11 \mathrm{~A})$ & $6005(10)$ & $4650(30)$ & $3748(18)$ & $110(3)$ \\
\hline $\mathrm{C}(12 \mathrm{~A})$ & $5308(9)$ & $4750(30)$ & $3879(15)$ & $123(3)$ \\
\hline $\mathrm{C}(13 \mathrm{~A})$ & $5116(7)$ & $6290(30)$ & $4483(15)$ & $126(3)$ \\
\hline $\mathrm{C}(14 \mathrm{~A})$ & $5621(9)$ & $7730(30)$ & $4956(15)$ & $124(3)$ \\
\hline $\mathrm{C}(15 \mathrm{~A})$ & $6318(9)$ & $7630(40)$ & $4820(20)$ & $111(3)$ \\
\hline $\mathrm{C}(16 \mathrm{~A})$ & $6889(16)$ & $8920(50)$ & $5790(20)$ & $107(5)$ \\
\hline $\mathrm{B}(1)$ & $8129(2)$ & $3265(5)$ & $3893(3)$ & $46(1)$ \\
\hline
\end{tabular}

Table S-5. Bond lengths $[\AA]$ and angles $\left[{ }^{\circ}\right]$ for $\mathbf{2 c}$.

\begin{tabular}{|l|l|}
\hline $\mathrm{C}(2)-\mathrm{N}(1)$ & $1.510(4)$ \\
\hline $\mathrm{C}(2)-\mathrm{H}(2 \mathrm{~A})$ & 0.9800 \\
\hline $\mathrm{C}(2)-\mathrm{H}(2 \mathrm{~B})$ & 0.9800 \\
\hline $\mathrm{C}(2)-\mathrm{H}(2 \mathrm{C})$ & 0.9800 \\
\hline $\mathrm{O}(1)-\mathrm{C}(4)$ & $1.317(4)$ \\
\hline $\mathrm{O}(1)-\mathrm{B}(1)$ & $1.482(4)$ \\
\hline $\mathrm{O}(2)-\mathrm{C}(4)$ & $1.209(4)$ \\
\hline $\mathrm{O}(3)-\mathrm{C}(6)$ & $1.318(4)$ \\
\hline $\mathrm{O}(3)-\mathrm{B}(1)$ & $1.491(5)$ \\
\hline $\mathrm{O}(4)-\mathrm{C}(6)$ & $1.213(4)$ \\
\hline $\mathrm{N}(1)-\mathrm{C}(3)$ & $1.501(4)$ \\
\hline $\mathrm{N}(1)-\mathrm{C}(5)$ & $1.504(3)$ \\
\hline $\mathrm{N}(1)-\mathrm{C}(1 \mathrm{~A})$ & $1.528(4)$ \\
\hline $\mathrm{N}(1)-\mathrm{C}(1)$ & $1.528(4)$ \\
\hline $\mathrm{C}(3)-\mathrm{C}(4)$ & $1.507(5)$ \\
\hline $\mathrm{C}(3)-\mathrm{H}(3 \mathrm{~A})$ & 0.9900 \\
\hline $\mathrm{C}(3)-\mathrm{H}(3 \mathrm{~B})$ & 0.9900 \\
\hline $\mathrm{C}(5)-\mathrm{C}(6)$ & $1.512(4)$ \\
\hline $\mathrm{C}(5)-\mathrm{H}(5 \mathrm{~A})$ & 0.9900 \\
\hline $\mathrm{C}(5)-\mathrm{H}(5 \mathrm{~B})$ & 0.9900 \\
\hline $\mathrm{C}(1)-\mathrm{C}(7)$ & $1.404(6)$ \\
\hline
\end{tabular}




\begin{tabular}{|l|l|}
\hline $\mathrm{C}(1)-\mathrm{B}(1)$ & $1.597(5)$ \\
\hline $\mathrm{C}(1)-\mathrm{H}(1)$ & 1.0000 \\
\hline $\mathrm{O}(5)-\mathrm{C}(9)$ & $1.355(9)$ \\
\hline $\mathrm{O}(5)-\mathrm{B}(1)$ & $1.471(6)$ \\
\hline $\mathrm{O}(6)-\mathrm{C}(9)$ & $1.182(7)$ \\
\hline $\mathrm{C}(7)-\mathrm{C}(8)$ & $1.323(9)$ \\
\hline $\mathrm{C}(7)-\mathrm{H}(7 \mathrm{~A})$ & 0.9500 \\
\hline $\mathrm{C}(8)-\mathrm{H}(8 \mathrm{~A})$ & 0.9500 \\
\hline $\mathrm{C}(8)-\mathrm{H}(8 \mathrm{~B})$ & 0.9500 \\
\hline $\mathrm{C}(9)-\mathrm{C}(10)$ & $1.501(6)$ \\
\hline $\mathrm{C}(1 \mathrm{~A})-\mathrm{C}(7 \mathrm{~A})$ & $1.387(11)$ \\
\hline $\mathrm{C}(1 \mathrm{~A})-\mathrm{B}(1)$ & $1.597(5)$ \\
\hline $\mathrm{C}(1 \mathrm{~A})-\mathrm{H}(1 \mathrm{~A})$ & 1.0000 \\
\hline $\mathrm{O}(5 \mathrm{~A})-\mathrm{C}(9 \mathrm{~A})$ & $1.23(2)$ \\
\hline $\mathrm{O}(5 \mathrm{~A})-\mathrm{B}(1)$ & $1.498(12)$ \\
\hline $\mathrm{O}(6 \mathrm{~A})-\mathrm{C}(9 \mathrm{~A})$ & $1.26(2)$ \\
\hline $\mathrm{C}(7 \mathrm{~A})-\mathrm{C}(8 \mathrm{~A})$ & $1.281(18)$ \\
\hline $\mathrm{C}(7 \mathrm{~A})-\mathrm{H}(7 \mathrm{AA})$ & 0.9500 \\
\hline $\mathrm{C}(8 \mathrm{~A})-\mathrm{H}(8 \mathrm{~A} 1)$ & 0.9500 \\
\hline $\mathrm{C}(8 \mathrm{~A})-\mathrm{H}(8 \mathrm{~A} 2)$ & 0.9500 \\
\hline $\mathrm{C}(9 \mathrm{~A})-\mathrm{C}(10 \mathrm{~A})$ & $1.508(10)$ \\
\hline $\mathrm{C}(10)-\mathrm{C}(11)$ & 1.3900 \\
\hline $\mathrm{C}(10)-\mathrm{C}(15)$ & 1.3900 \\
\hline $\mathrm{C}(11)-\mathrm{C}(12)$ & 1.3900 \\
\hline $\mathrm{C}(11)-\mathrm{H}(11 \mathrm{~A})$ & 0.9500 \\
\hline $\mathrm{C}(12)-\mathrm{C}(13)$ & 1.3900 \\
\hline $\mathrm{C}(12)-\mathrm{H}(12 \mathrm{~A})$ & 0.9500 \\
\hline $\mathrm{C}(13)-\mathrm{C}(14)$ & 1.3900 \\
\hline $\mathrm{C}(13)-\mathrm{H}(13 \mathrm{~A})$ & 0.9500 \\
\hline $\mathrm{C}(14)-\mathrm{C}(15)$ & 1.3900 \\
\hline $\mathrm{C}(14)-\mathrm{H}(14 \mathrm{~A})$ & 0.9500 \\
\hline $\mathrm{C}(15)-\mathrm{C}(16)$ & $1.477(18)$ \\
\hline $\mathrm{C}(16)-\mathrm{H}(16 \mathrm{~A})$ & 0.9800 \\
\hline $\mathrm{C}(16)-\mathrm{H}(16 \mathrm{~B})$ & 0.9800 \\
\hline $\mathrm{C}(16)-\mathrm{H}(16 \mathrm{C})$ & 0.9800 \\
\hline $\mathrm{C}(10 \mathrm{~A})-\mathrm{C}(11 \mathrm{~A})$ & 1.3900 \\
\hline $\mathrm{C}(10 \mathrm{~A})-\mathrm{C}(15 \mathrm{~A})$ & 1.3900 \\
\hline $\mathrm{C}(11 \mathrm{~A})-\mathrm{C}(12 \mathrm{~A})$ & 1.3900 \\
\hline $\mathrm{C}(11 \mathrm{~A})-\mathrm{H}(11 \mathrm{~B})$ & 0.9500 \\
\hline $\mathrm{C}(12 \mathrm{~A})-\mathrm{C}(13 \mathrm{~A})$ & 1.3900 \\
\hline $\mathrm{C}(12 \mathrm{~A})-\mathrm{H}(12 \mathrm{~B})$ & 0.9500 \\
\hline $\mathrm{C}(13 \mathrm{~A})-\mathrm{C}(14 \mathrm{~A})$ & 1.3900 \\
\hline $\mathrm{C}(13 \mathrm{~A})-\mathrm{H}(13 \mathrm{~B})$ & 0.9500 \\
\hline $\mathrm{C}(14 \mathrm{~A})-\mathrm{C}(15 \mathrm{~A})$ & 1.3900 \\
\hline $\mathrm{C}(14 \mathrm{~A})-\mathrm{H}(14 \mathrm{~B})$ & 0.9500 \\
\hline $\mathrm{C}(15 \mathrm{~A})-\mathrm{C}(16 \mathrm{~A})$ & $1.69(4)$ \\
\hline & \\
\hline
\end{tabular}




\begin{tabular}{|l|l|}
\hline $\mathrm{C}(16 \mathrm{~A})-\mathrm{H}(16 \mathrm{D})$ & 0.9800 \\
\hline $\mathrm{C}(16 \mathrm{~A})-\mathrm{H}(16 \mathrm{E})$ & 0.9800 \\
\hline $\mathrm{C}(16 \mathrm{~A})-\mathrm{H}(16 \mathrm{~F})$ & 0.9800 \\
\hline $\mathrm{N}(1)-\mathrm{C}(2)-\mathrm{H}(2 \mathrm{~A})$ & 109.5 \\
\hline $\mathrm{N}(1)-\mathrm{C}(2)-\mathrm{H}(2 \mathrm{~B})$ & 109.5 \\
\hline $\mathrm{H}(2 \mathrm{~A})-\mathrm{C}(2)-\mathrm{H}(2 \mathrm{~B})$ & 109.5 \\
\hline $\mathrm{N}(1)-\mathrm{C}(2)-\mathrm{H}(2 \mathrm{C})$ & 109.5 \\
\hline $\mathrm{H}(2 \mathrm{~A})-\mathrm{C}(2)-\mathrm{H}(2 \mathrm{C})$ & 109.5 \\
\hline $\mathrm{H}(2 \mathrm{~B})-\mathrm{C}(2)-\mathrm{H}(2 \mathrm{C})$ & 109.5 \\
\hline $\mathrm{C}(4)-\mathrm{O}(1)-\mathrm{B}(1)$ & $121.0(2)$ \\
\hline $\mathrm{C}(6)-\mathrm{O}(3)-\mathrm{B}(1)$ & $122.5(2)$ \\
\hline $\mathrm{C}(3)-\mathrm{N}(1)-\mathrm{C}(5)$ & $110.8(2)$ \\
\hline $\mathrm{C}(3)-\mathrm{N}(1)-\mathrm{C}(2)$ & $106.9(2)$ \\
\hline $\mathrm{C}(5)-\mathrm{N}(1)-\mathrm{C}(2)$ & $107.4(2)$ \\
\hline $\mathrm{C}(3)-\mathrm{N}(1)-\mathrm{C}(1 \mathrm{~A})$ & $109.8(2)$ \\
\hline $\mathrm{C}(5)-\mathrm{N}(1)-\mathrm{C}(1 \mathrm{~A})$ & $109.5(2)$ \\
\hline $\mathrm{C}(2)-\mathrm{N}(1)-\mathrm{C}(1 \mathrm{~A})$ & $112.3(2)$ \\
\hline $\mathrm{C}(3)-\mathrm{N}(1)-\mathrm{C}(1)$ & $109.8(2)$ \\
\hline $\mathrm{C}(5)-\mathrm{N}(1)-\mathrm{C}(1)$ & $109.5(2)$ \\
\hline $\mathrm{C}(2)-\mathrm{N}(1)-\mathrm{C}(1)$ & $112.3(2)$ \\
\hline $\mathrm{N}(1)-\mathrm{C}(3)-\mathrm{C}(4)$ & $118.1(2)$ \\
\hline $\mathrm{N}(1)-\mathrm{C}(3)-\mathrm{H}(3 \mathrm{~A})$ & 107.8 \\
\hline $\mathrm{C}(4)-\mathrm{C}(3)-\mathrm{H}(3 \mathrm{~A})$ & 107.8 \\
\hline $\mathrm{N}(1)-\mathrm{C}(3)-\mathrm{H}(3 \mathrm{~B})$ & 107.8 \\
\hline $\mathrm{C}(4)-\mathrm{C}(3)-\mathrm{H}(3 \mathrm{~B})$ & 107.8 \\
\hline $\mathrm{H}(3 \mathrm{~A})-\mathrm{C}(3)-\mathrm{H}(3 \mathrm{~B})$ & 107.1 \\
\hline $\mathrm{O}(2)-\mathrm{C}(4)-\mathrm{O}(1)$ & $121.9(3)$ \\
\hline $\mathrm{O}(2)-\mathrm{C}(4)-\mathrm{C}(3)$ & $118.2(3)$ \\
\hline $\mathrm{O}(1)-\mathrm{C}(4)-\mathrm{C}(3)$ & $119.6(3)$ \\
\hline $\mathrm{N}(1)-\mathrm{C}(5)-\mathrm{C}(6)$ & $116.9(2)$ \\
\hline $\mathrm{N}(1)-\mathrm{C}(5)-\mathrm{H}(5 \mathrm{~A})$ & 108.1 \\
\hline $\mathrm{C}(6)-\mathrm{C}(5)-\mathrm{H}(5 \mathrm{~A})$ & 108.1 \\
\hline $\mathrm{N}(1)-\mathrm{C}(5)-\mathrm{H}(5 \mathrm{~B})$ & 108.1 \\
\hline $\mathrm{C}(6)-\mathrm{C}(5)-\mathrm{H}(5 \mathrm{~B})$ & 108.1 \\
\hline $\mathrm{H}(5 \mathrm{~A})-\mathrm{C}(5)-\mathrm{H}(5 \mathrm{~B})$ & 107.3 \\
\hline $\mathrm{O}(4)-\mathrm{C}(6)-\mathrm{O}(3)$ & $122.3(3)$ \\
\hline $\mathrm{O}(4)-\mathrm{C}(6)-\mathrm{C}(5)$ & $117.8(3)$ \\
\hline $\mathrm{O}(3)-\mathrm{C}(6)-\mathrm{C}(5)$ & $119.7(3)$ \\
\hline $\mathrm{C}(7)-\mathrm{C}(1)-\mathrm{N}(1)$ & $115.7(3)$ \\
\hline $\mathrm{C}(7)-\mathrm{C}(1)-\mathrm{B}(1)$ & $116.6(4)$ \\
\hline $\mathrm{N}(1)-\mathrm{C}(1)-\mathrm{B}(1)$ & $105.0(3)$ \\
\hline $\mathrm{C}(7)-\mathrm{C}(1)-\mathrm{H}(1)$ & 106.3 \\
\hline $\mathrm{N}(1)-\mathrm{C}(1)-\mathrm{H}(1)$ & 106.3 \\
\hline $\mathrm{B}(1)-\mathrm{C}(1)-\mathrm{H}(1)$ & 106.3 \\
\hline $\mathrm{C}(9)-\mathrm{O}(5)-\mathrm{B}(1)$ & $120.5(4)$ \\
\hline $\mathrm{C}(8)-\mathrm{C}(7)-\mathrm{C}(1)$ & $126.1(6)$ \\
\hline & \\
\hline
\end{tabular}




\begin{tabular}{|l|l|}
\hline $\mathrm{C}(8)-\mathrm{C}(7)-\mathrm{H}(7 \mathrm{~A})$ & 116.9 \\
\hline $\mathrm{C}(1)-\mathrm{C}(7)-\mathrm{H}(7 \mathrm{~A})$ & 116.9 \\
\hline $\mathrm{C}(7)-\mathrm{C}(8)-\mathrm{H}(8 \mathrm{~A})$ & 120.0 \\
\hline $\mathrm{C}(7)-\mathrm{C}(8)-\mathrm{H}(8 \mathrm{~B})$ & 120.0 \\
\hline $\mathrm{H}(8 \mathrm{~A})-\mathrm{C}(8)-\mathrm{H}(8 \mathrm{~B})$ & 120.0 \\
\hline $\mathrm{O}(6)-\mathrm{C}(9)-\mathrm{O}(5)$ & $125.5(5)$ \\
\hline $\mathrm{O}(6)-\mathrm{C}(9)-\mathrm{C}(10)$ & $125.9(6)$ \\
\hline $\mathrm{O}(5)-\mathrm{C}(9)-\mathrm{C}(10)$ & $108.2(7)$ \\
\hline $\mathrm{C}(7 \mathrm{~A})-\mathrm{C}(1 \mathrm{~A})-\mathrm{N}(1)$ & $117.8(5)$ \\
\hline $\mathrm{C}(7 \mathrm{~A})-\mathrm{C}(1 \mathrm{~A})-\mathrm{B}(1)$ & $121.5(5)$ \\
\hline $\mathrm{N}(1)-\mathrm{C}(1 \mathrm{~A})-\mathrm{B}(1)$ & $105.0(3)$ \\
\hline $\mathrm{C}(7 \mathrm{~A})-\mathrm{C}(1 \mathrm{~A})-\mathrm{H}(1 \mathrm{~A})$ & 103.4 \\
\hline $\mathrm{N}(1)-\mathrm{C}(1 \mathrm{~A})-\mathrm{H}(1 \mathrm{~A})$ & 103.4 \\
\hline $\mathrm{B}(1)-\mathrm{C}(1 \mathrm{~A})-\mathrm{H}(1 \mathrm{~A})$ & 103.4 \\
\hline $\mathrm{C}(9 \mathrm{~A})-\mathrm{O}(5 \mathrm{~A})-\mathrm{B}(1)$ & $122.6(11)$ \\
\hline $\mathrm{C}(8 \mathrm{~A})-\mathrm{C}(7 \mathrm{~A})-\mathrm{C}(1 \mathrm{~A})$ & $129.4(12)$ \\
\hline $\mathrm{C}(8 \mathrm{~A})-\mathrm{C}(7 \mathrm{~A})-\mathrm{H}(7 \mathrm{AA})$ & 115.3 \\
\hline $\mathrm{C}(1 \mathrm{~A})-\mathrm{C}(7 \mathrm{~A})-\mathrm{H}(7 \mathrm{AA})$ & 115.3 \\
\hline $\mathrm{C}(7 \mathrm{~A})-\mathrm{C}(8 \mathrm{~A})-\mathrm{H}(8 \mathrm{~A} 1)$ & 120.0 \\
\hline $\mathrm{C}(7 \mathrm{~A})-\mathrm{C}(8 \mathrm{~A})-\mathrm{H}(8 \mathrm{~A} 2)$ & 120.0 \\
\hline $\mathrm{H}(8 \mathrm{~A} 1)-\mathrm{C}(8 \mathrm{~A})-\mathrm{H}(8 \mathrm{~A} 2)$ & 120.0 \\
\hline $\mathrm{O}(5 \mathrm{~A})-\mathrm{C}(9 \mathrm{~A})-\mathrm{O}(6 \mathrm{~A})$ & $118.2(14)$ \\
\hline $\mathrm{O}(5 \mathrm{~A})-\mathrm{C}(9 \mathrm{~A})-\mathrm{C}(10 \mathrm{~A})$ & $114.7(17)$ \\
\hline $\mathrm{O}(6 \mathrm{~A})-\mathrm{C}(9 \mathrm{~A})-\mathrm{C}(10 \mathrm{~A})$ & $126.5(18)$ \\
\hline $\mathrm{C}(11)-\mathrm{C}(10)-\mathrm{C}(15)$ & 120.0 \\
\hline $\mathrm{C}(11)-\mathrm{C}(10)-\mathrm{C}(9)$ & $122.2(6)$ \\
\hline $\mathrm{C}(15)-\mathrm{C}(10)-\mathrm{C}(9)$ & $117.8(6)$ \\
\hline $\mathrm{C}(12)-\mathrm{C}(11)-\mathrm{C}(10)$ & 120.0 \\
\hline $\mathrm{C}(12)-\mathrm{C}(11)-\mathrm{H}(11 \mathrm{~A})$ & 120.0 \\
\hline $\mathrm{C}(10)-\mathrm{C}(11)-\mathrm{H}(11 \mathrm{~A})$ & 120.0 \\
\hline $\mathrm{C}(11)-\mathrm{C}(12)-\mathrm{C}(13)$ & 120.0 \\
\hline $\mathrm{C}(11)-\mathrm{C}(12)-\mathrm{H}(12 \mathrm{~A})$ & 120.0 \\
\hline $\mathrm{C}(13)-\mathrm{C}(12)-\mathrm{H}(12 \mathrm{~A})$ & 120.0 \\
\hline $\mathrm{C}(14)-\mathrm{C}(13)-\mathrm{C}(12)$ & 120.0 \\
\hline $\mathrm{C}(14)-\mathrm{C}(13)-\mathrm{H}(13 \mathrm{~A})$ & 120.0 \\
\hline $\mathrm{C}(12)-\mathrm{C}(13)-\mathrm{H}(13 \mathrm{~A})$ & 120.0 \\
\hline $\mathrm{C}(13)-\mathrm{C}(14)-\mathrm{C}(15)$ & 120.0 \\
\hline $\mathrm{C}(13)-\mathrm{C}(14)-\mathrm{H}(14 \mathrm{~A})$ & 120.0 \\
\hline $\mathrm{C}(15)-\mathrm{C}(14)-\mathrm{H}(14 \mathrm{~A})$ & 120.0 \\
\hline $\mathrm{C}(14)-\mathrm{C}(15)-\mathrm{C}(10)$ & 120.0 \\
\hline $\mathrm{C}(14)-\mathrm{C}(15)-\mathrm{C}(16)$ & $114.3(8)$ \\
\hline $\mathrm{C}(10)-\mathrm{C}(15)-\mathrm{C}(16)$ & $125.6(8)$ \\
\hline $\mathrm{C}(15)-\mathrm{C}(16)-\mathrm{H}(16 \mathrm{~A})$ & \\
\hline $\mathrm{C}(15)-\mathrm{C}(16)-\mathrm{H}(16 \mathrm{~B})$ & \\
\hline $\mathrm{H}(16 \mathrm{~A})-\mathrm{C}(16)-\mathrm{H}(16 \mathrm{~B})$ & \\
\hline $\mathrm{C}(15)-\mathrm{C}(16)-\mathrm{H}(16 \mathrm{C})$ & \\
\hline
\end{tabular}




\begin{tabular}{|c|c|}
\hline $\mathrm{H}(16 \mathrm{~A})-\mathrm{C}(16)-\mathrm{H}(16 \mathrm{C})$ & 109.5 \\
\hline $\mathrm{H}(16 \mathrm{~B})-\mathrm{C}(16)-\mathrm{H}(16 \mathrm{C})$ & 109.5 \\
\hline$C(11 A)-C(10 A)-C(15 A)$ & 120.0 \\
\hline$C(11 A)-C(10 A)-C(9 A)$ & $107.9(16)$ \\
\hline $\mathrm{C}(15 \mathrm{~A})-\mathrm{C}(10 \mathrm{~A})-\mathrm{C}(9 \mathrm{~A})$ & $132.0(16)$ \\
\hline $\mathrm{C}(12 \mathrm{~A})-\mathrm{C}(11 \mathrm{~A})-\mathrm{C}(10 \mathrm{~A})$ & 120.0 \\
\hline $\mathrm{C}(12 \mathrm{~A})-\mathrm{C}(11 \mathrm{~A})-\mathrm{H}(11 \mathrm{~B})$ & 120.0 \\
\hline $\mathrm{C}(10 \mathrm{~A})-\mathrm{C}(11 \mathrm{~A})-\mathrm{H}(11 \mathrm{~B})$ & 120.0 \\
\hline $\mathrm{C}(11 \mathrm{~A})-\mathrm{C}(12 \mathrm{~A})-\mathrm{C}(13 \mathrm{~A})$ & 120.0 \\
\hline $\mathrm{C}(11 \mathrm{~A})-\mathrm{C}(12 \mathrm{~A})-\mathrm{H}(12 \mathrm{~B})$ & 120.0 \\
\hline $\mathrm{C}(13 \mathrm{~A})-\mathrm{C}(12 \mathrm{~A})-\mathrm{H}(12 \mathrm{~B})$ & 120.0 \\
\hline$C(14 \mathrm{~A})-\mathrm{C}(13 \mathrm{~A})-\mathrm{C}(12 \mathrm{~A})$ & 120.0 \\
\hline $\mathrm{C}(14 \mathrm{~A})-\mathrm{C}(13 \mathrm{~A})-\mathrm{H}(13 \mathrm{~B})$ & 120.0 \\
\hline $\mathrm{C}(12 \mathrm{~A})-\mathrm{C}(13 \mathrm{~A})-\mathrm{H}(13 \mathrm{~B})$ & 120.0 \\
\hline$C(13 A)-C(14 A)-C(15 A)$ & 120.0 \\
\hline $\mathrm{C}(13 \mathrm{~A})-\mathrm{C}(14 \mathrm{~A})-\mathrm{H}(14 \mathrm{~B})$ & 120.0 \\
\hline $\mathrm{C}(15 \mathrm{~A})-\mathrm{C}(14 \mathrm{~A})-\mathrm{H}(14 \mathrm{~B})$ & 120.0 \\
\hline $\mathrm{C}(14 \mathrm{~A})-\mathrm{C}(15 \mathrm{~A})-\mathrm{C}(10 \mathrm{~A})$ & 120.0 \\
\hline$C(14 A)-C(15 A)-C(16 A)$ & $110.6(14)$ \\
\hline$C(10 A)-C(15 A)-C(16 A)$ & $125.5(14)$ \\
\hline $\mathrm{C}(15 \mathrm{~A})-\mathrm{C}(16 \mathrm{~A})-\mathrm{H}(16 \mathrm{D})$ & 109.5 \\
\hline $\mathrm{C}(15 \mathrm{~A})-\mathrm{C}(16 \mathrm{~A})-\mathrm{H}(16 \mathrm{E})$ & 109.5 \\
\hline $\mathrm{H}(16 \mathrm{D})-\mathrm{C}(16 \mathrm{~A})-\mathrm{H}(16 \mathrm{E})$ & 109.5 \\
\hline $\mathrm{C}(15 \mathrm{~A})-\mathrm{C}(16 \mathrm{~A})-\mathrm{H}(16 \mathrm{~F})$ & 109.5 \\
\hline $\mathrm{H}(16 \mathrm{D})-\mathrm{C}(16 \mathrm{~A})-\mathrm{H}(16 \mathrm{~F})$ & 109.5 \\
\hline $\mathrm{H}(16 \mathrm{E})-\mathrm{C}(16 \mathrm{~A})-\mathrm{H}(16 \mathrm{~F})$ & 109.5 \\
\hline $\mathrm{O}(5)-\mathrm{B}(1)-\mathrm{O}(1)$ & $110.7(3)$ \\
\hline $\mathrm{O}(5)-\mathrm{B}(1)-\mathrm{O}(3)$ & $113.4(3)$ \\
\hline $\mathrm{O}(1)-\mathrm{B}(1)-\mathrm{O}(3)$ & $107.7(3)$ \\
\hline $\mathrm{O}(1)-\mathrm{B}(1)-\mathrm{O}(5 \mathrm{~A})$ & $112.2(5)$ \\
\hline $\mathrm{O}(3)-\mathrm{B}(1)-\mathrm{O}(5 \mathrm{~A})$ & $89.3(5)$ \\
\hline $\mathrm{O}(5)-\mathrm{B}(1)-\mathrm{C}(1)$ & $104.2(3)$ \\
\hline $\mathrm{O}(1)-\mathrm{B}(1)-\mathrm{C}(1)$ & $109.6(3)$ \\
\hline $\mathrm{O}(3)-\mathrm{B}(1)-\mathrm{C}(1)$ & $111.1(3)$ \\
\hline $\mathrm{O}(1)-\mathrm{B}(1)-\mathrm{C}(1 \mathrm{~A})$ & $109.6(3)$ \\
\hline $\mathrm{O}(3)-\mathrm{B}(1)-\mathrm{C}(1 \mathrm{~A})$ & $111.1(3)$ \\
\hline $\mathrm{O}(5 \mathrm{~A})-\mathrm{B}(1)-\mathrm{C}(1 \mathrm{~A})$ & 124.26 \\
\hline $\mathrm{C}(13)-\mathrm{C}(14)-\mathrm{H}(14 \mathrm{~A})$ & 120.0 \\
\hline $\mathrm{C}(15)-\mathrm{C}(14)-\mathrm{H}(14 \mathrm{~A})$ & 120.0 \\
\hline $\mathrm{C}(14)-\mathrm{C}(15)-\mathrm{C}(10)$ & 120.0 \\
\hline $\mathrm{C}(14)-\mathrm{C}(15)-\mathrm{C}(16)$ & $114.3(8)$ \\
\hline $\mathrm{C}(10)-\mathrm{C}(15)-\mathrm{C}(16)$ & $125.6(8)$ \\
\hline $\mathrm{C}(15)-\mathrm{C}(16)-\mathrm{H}(16 \mathrm{~A})$ & 109.5 \\
\hline $\mathrm{C}(15)-\mathrm{C}(16)-\mathrm{H}(16 \mathrm{~B})$ & 109.5 \\
\hline $\mathrm{H}(16 \mathrm{~A})-\mathrm{C}(16)-\mathrm{H}(16 \mathrm{~B})$ & 109.5 \\
\hline $\mathrm{C}(15)-\mathrm{C}(16)-\mathrm{H}(16 \mathrm{C})$ & 109.5 \\
\hline
\end{tabular}




\begin{tabular}{|c|c|}
\hline $\mathrm{H}(16 \mathrm{~A})-\mathrm{C}(16)-\mathrm{H}(16 \mathrm{C})$ & 109.5 \\
\hline $\mathrm{H}(16 \mathrm{~B})-\mathrm{C}(16)-\mathrm{H}(16 \mathrm{C})$ & 109.5 \\
\hline$C(11 \mathrm{~A})-\mathrm{C}(10 \mathrm{~A})-\mathrm{C}(15 \mathrm{~A})$ & 120.0 \\
\hline $\mathrm{C}(11 \mathrm{~A})-\mathrm{C}(10 \mathrm{~A})-\mathrm{C}(9 \mathrm{~A})$ & $107.9(16)$ \\
\hline $\mathrm{C}(15 \mathrm{~A})-\mathrm{C}(10 \mathrm{~A})-\mathrm{C}(9 \mathrm{~A})$ & $132.0(16)$ \\
\hline$C(12 A)-C(11 A)-C(10 A)$ & 120.0 \\
\hline $\mathrm{C}(12 \mathrm{~A})-\mathrm{C}(11 \mathrm{~A})-\mathrm{H}(11 \mathrm{~B})$ & 120.0 \\
\hline $\mathrm{C}(10 \mathrm{~A})-\mathrm{C}(11 \mathrm{~A})-\mathrm{H}(11 \mathrm{~B})$ & 120.0 \\
\hline $\mathrm{C}(11 \mathrm{~A})-\mathrm{C}(12 \mathrm{~A})-\mathrm{C}(13 \mathrm{~A})$ & 120.0 \\
\hline $\mathrm{C}(11 \mathrm{~A})-\mathrm{C}(12 \mathrm{~A})-\mathrm{H}(12 \mathrm{~B})$ & 120.0 \\
\hline $\mathrm{C}(13 \mathrm{~A})-\mathrm{C}(12 \mathrm{~A})-\mathrm{H}(12 \mathrm{~B})$ & 120.0 \\
\hline $\mathrm{C}(14 \mathrm{~A})-\mathrm{C}(13 \mathrm{~A})-\mathrm{C}(12 \mathrm{~A})$ & 120.0 \\
\hline $\mathrm{C}(14 \mathrm{~A})-\mathrm{C}(13 \mathrm{~A})-\mathrm{H}(13 \mathrm{~B})$ & 120.0 \\
\hline $\mathrm{C}(12 \mathrm{~A})-\mathrm{C}(13 \mathrm{~A})-\mathrm{H}(13 \mathrm{~B})$ & 120.0 \\
\hline$C(13 A)-C(14 A)-C(15 A)$ & 120.0 \\
\hline $\mathrm{C}(13 \mathrm{~A})-\mathrm{C}(14 \mathrm{~A})-\mathrm{H}(14 \mathrm{~B})$ & 120.0 \\
\hline $\mathrm{C}(15 \mathrm{~A})-\mathrm{C}(14 \mathrm{~A})-\mathrm{H}(14 \mathrm{~B})$ & 120.0 \\
\hline$C(14 A)-C(15 A)-C(10 A)$ & 120.0 \\
\hline$C(14 A)-C(15 A)-C(16 A)$ & $110.6(14)$ \\
\hline$C(10 A)-C(15 A)-C(16 A)$ & $125.5(14)$ \\
\hline $\mathrm{C}(15 \mathrm{~A})-\mathrm{C}(16 \mathrm{~A})-\mathrm{H}(16 \mathrm{D})$ & 109.5 \\
\hline $\mathrm{C}(15 \mathrm{~A})-\mathrm{C}(16 \mathrm{~A})-\mathrm{H}(16 \mathrm{E})$ & 109.5 \\
\hline $\mathrm{H}(16 \mathrm{D})-\mathrm{C}(16 \mathrm{~A})-\mathrm{H}(16 \mathrm{E})$ & 109.5 \\
\hline $\mathrm{C}(15 \mathrm{~A})-\mathrm{C}(16 \mathrm{~A})-\mathrm{H}(16 \mathrm{~F})$ & 109.5 \\
\hline $\mathrm{H}(16 \mathrm{D})-\mathrm{C}(16 \mathrm{~A})-\mathrm{H}(16 \mathrm{~F})$ & 109.5 \\
\hline $\mathrm{H}(16 \mathrm{E})-\mathrm{C}(16 \mathrm{~A})-\mathrm{H}(16 \mathrm{~F})$ & 109.5 \\
\hline $\mathrm{O}(5)-\mathrm{B}(1)-\mathrm{O}(1)$ & $110.7(3)$ \\
\hline $\mathrm{O}(5)-\mathrm{B}(1)-\mathrm{O}(3)$ & $113.4(3)$ \\
\hline $\mathrm{O}(1)-\mathrm{B}(1)-\mathrm{O}(3)$ & $107.7(3)$ \\
\hline $\mathrm{O}(1)-\mathrm{B}(1)-\mathrm{O}(5 \mathrm{~A})$ & $112.2(5)$ \\
\hline $\mathrm{O}(3)-\mathrm{B}(1)-\mathrm{O}(5 \mathrm{~A})$ & $89.3(5)$ \\
\hline $\mathrm{O}(5)-\mathrm{B}(1)-\mathrm{C}(1)$ & 104.2(3) \\
\hline $\mathrm{O}(1)-\mathrm{B}(1)-\mathrm{C}(1)$ & $109.6(3)$ \\
\hline $\mathrm{O}(3)-\mathrm{B}(1)-\mathrm{C}(1)$ & 111.1(3) \\
\hline $\mathrm{O}(1)-\mathrm{B}(1)-\mathrm{C}(1 \mathrm{~A})$ & $109.6(3)$ \\
\hline $\mathrm{O}(3)-\mathrm{B}(1)-\mathrm{C}(1 \mathrm{~A})$ & $111.1(3)$ \\
\hline $\mathrm{O}(5 \mathrm{~A})-\mathrm{B}(1)-\mathrm{C}(1 \mathrm{~A})$ & $124.2(6$ \\
\hline $\mathrm{C}(13)-\mathrm{C}(14)-\mathrm{H}(14 \mathrm{~A})$ & 120.0 \\
\hline $\mathrm{C}(15)-\mathrm{C}(14)-\mathrm{H}(14 \mathrm{~A})$ & 120.0 \\
\hline $\mathrm{C}(14)-\mathrm{C}(15)-\mathrm{C}(10)$ & 120.0 \\
\hline $\mathrm{C}(14)-\mathrm{C}(15)-\mathrm{C}(16)$ & $114.3(8)$ \\
\hline $\mathrm{C}(10)-\mathrm{C}(15)-\mathrm{C}(16)$ & $125.6(8)$ \\
\hline $\mathrm{C}(15)-\mathrm{C}(16)-\mathrm{H}(16 \mathrm{~A})$ & 109.5 \\
\hline $\mathrm{C}(15)-\mathrm{C}(16)-\mathrm{H}(16 \mathrm{~B})$ & 109.5 \\
\hline $\mathrm{H}(16 \mathrm{~A})-\mathrm{C}(16)-\mathrm{H}(16 \mathrm{~B})$ & 109.5 \\
\hline $\mathrm{C}(15)-\mathrm{C}(16)-\mathrm{H}(16 \mathrm{C})$ & 109.5 \\
\hline
\end{tabular}




\begin{tabular}{|l|l|}
\hline$H(16 \mathrm{~A})-\mathrm{C}(16)-\mathrm{H}(16 \mathrm{C})$ & 109.5 \\
\hline $\mathrm{H}(16 \mathrm{~B})-\mathrm{C}(16)-\mathrm{H}(16 \mathrm{C})$ & 109.5 \\
\hline $\mathrm{C}(11 \mathrm{~A})-\mathrm{C}(10 \mathrm{~A})-\mathrm{C}(15 \mathrm{~A})$ & 120.0 \\
\hline $\mathrm{C}(11 \mathrm{~A})-\mathrm{C}(10 \mathrm{~A})-\mathrm{C}(9 \mathrm{~A})$ & $107.9(16)$ \\
\hline $\mathrm{C}(15 \mathrm{~A})-\mathrm{C}(10 \mathrm{~A})-\mathrm{C}(9 \mathrm{~A})$ & $132.0(16)$ \\
\hline $\mathrm{C}(12 \mathrm{~A})-\mathrm{C}(11 \mathrm{~A})-\mathrm{C}(10 \mathrm{~A})$ & 120.0 \\
\hline $\mathrm{C}(12 \mathrm{~A})-\mathrm{C}(11 \mathrm{~A})-\mathrm{H}(11 \mathrm{~B})$ & 120.0 \\
\hline $\mathrm{C}(10 \mathrm{~A})-\mathrm{C}(11 \mathrm{~A})-\mathrm{H}(11 \mathrm{~B})$ & 120.0 \\
\hline $\mathrm{C}(11 \mathrm{~A})-\mathrm{C}(12 \mathrm{~A})-\mathrm{C}(13 \mathrm{~A})$ & 120.0 \\
\hline $\mathrm{C}(11 \mathrm{~A})-\mathrm{C}(12 \mathrm{~A})-\mathrm{H}(12 \mathrm{~B})$ & 120.0 \\
\hline $\mathrm{C}(13 \mathrm{~A})-\mathrm{C}(12 \mathrm{~A})-\mathrm{H}(12 \mathrm{~B})$ & 120.0 \\
\hline $\mathrm{C}(14 \mathrm{~A})-\mathrm{C}(13 \mathrm{~A})-\mathrm{C}(12 \mathrm{~A})$ & 120.0 \\
\hline $\mathrm{C}(14 \mathrm{~A})-\mathrm{C}(13 \mathrm{~A})-\mathrm{H}(13 \mathrm{~B})$ & 120.0 \\
\hline $\mathrm{C}(12 \mathrm{~A})-\mathrm{C}(13 \mathrm{~A})-\mathrm{H}(13 \mathrm{~B})$ & 120.0 \\
\hline $\mathrm{C}(13 \mathrm{~A})-\mathrm{C}(14 \mathrm{~A})-\mathrm{C}(15 \mathrm{~A})$ & 120.0 \\
\hline $\mathrm{C}(13 \mathrm{~A})-\mathrm{C}(14 \mathrm{~A})-\mathrm{H}(14 \mathrm{~B})$ & 120.0 \\
\hline $\mathrm{C}(15 \mathrm{~A})-\mathrm{C}(14 \mathrm{~A})-\mathrm{H}(14 \mathrm{~B})$ & 120.0 \\
\hline $\mathrm{C}(14 \mathrm{~A})-\mathrm{C}(15 \mathrm{~A})-\mathrm{C}(10 \mathrm{~A})$ & 120.0 \\
\hline $\mathrm{C}(14 \mathrm{~A})-\mathrm{C}(15 \mathrm{~A})-\mathrm{C}(16 \mathrm{~A})$ & $110.6(14)$ \\
\hline $\mathrm{C}(10 \mathrm{~A})-\mathrm{C}(15 \mathrm{~A})-\mathrm{C}(16 \mathrm{~A})$ & $125.5(14)$ \\
\hline $\mathrm{C}(15 \mathrm{~A})-\mathrm{C}(16 \mathrm{~A})-\mathrm{H}(16 \mathrm{D})$ & 109.5 \\
\hline $\mathrm{C}(15 \mathrm{~A})-\mathrm{C}(16 \mathrm{~A})-\mathrm{H}(16 \mathrm{E})$ & 109.5 \\
\hline $\mathrm{H}(16 \mathrm{D})-\mathrm{C}(16 \mathrm{~A})-\mathrm{H}(16 \mathrm{E})$ & 109.5 \\
\hline $\mathrm{C}(15 \mathrm{~A})-\mathrm{C}(16 \mathrm{~A})-\mathrm{H}(16 \mathrm{~F})$ & 109.5 \\
\hline $\mathrm{H}(16 \mathrm{D})-\mathrm{C}(16 \mathrm{~A})-\mathrm{H}(16 \mathrm{~F})$ & 109.5 \\
\hline $\mathrm{H}(16 \mathrm{E})-\mathrm{C}(16 \mathrm{~A})-\mathrm{H}(16 \mathrm{~F})$ & 109.5 \\
\hline $\mathrm{O}(5)-\mathrm{B}(1)-\mathrm{O}(1)$ & $110.7(3)$ \\
\hline $\mathrm{O}(5)-\mathrm{B}(1)-\mathrm{O}(3)$ & $113.4(3)$ \\
\hline $\mathrm{O}(1)-\mathrm{B}(1)-\mathrm{O}(3)$ & $107.7(3)$ \\
\hline $\mathrm{O}(1)-\mathrm{B}(1)-\mathrm{O}(5 \mathrm{~A})$ & $112.2(5)$ \\
\hline $\mathrm{O}(3)-\mathrm{B}(1)-\mathrm{O}(5 \mathrm{~A})$ & $89.3(5)$ \\
\hline $\mathrm{O}(5)-\mathrm{B}(1)-\mathrm{C}(1)$ & $104.2(3)$ \\
\hline $\mathrm{O}(1)-\mathrm{B}(1)-\mathrm{C}(1)$ & $109.6(3)$ \\
\hline $\mathrm{O}(3)-\mathrm{B}(1)-\mathrm{C}(1)$ & $11.1(3)$ \\
\hline $\mathrm{O}(1)-\mathrm{B}(1)-\mathrm{C}(1 \mathrm{~A})$ & $109.6(3)$ \\
\hline $\mathrm{O}(3)-\mathrm{B}(1)-\mathrm{C}(1 \mathrm{~A})$ & $111.1(3)$ \\
\hline $\mathrm{O}(5 \mathrm{~A})-\mathrm{B}(1)-\mathrm{C}(1 \mathrm{~A})$ & $124.2(6)$ \\
\hline $\mathrm{C}(15)-\mathrm{C}(16)-\mathrm{H}(16 \mathrm{C})$ & 109.5 \\
\hline $\mathrm{H}(16 \mathrm{~A})-\mathrm{C}(16)-\mathrm{H}(16 \mathrm{C})$ & 109.5 \\
\hline $\mathrm{H}(16 \mathrm{~B})-\mathrm{C}(16)-\mathrm{H}(16 \mathrm{C})$ & 109.5 \\
\hline $\mathrm{C}(11 \mathrm{~A})-\mathrm{C}(10 \mathrm{~A})-\mathrm{C}(15 \mathrm{~A})$ & 120.0 \\
\hline $\mathrm{C}(11 \mathrm{~A})-\mathrm{C}(10 \mathrm{~A})-\mathrm{C}(9 \mathrm{~A})$ & $132.0(16)$ \\
\hline $\mathrm{C}(15 \mathrm{~A})-\mathrm{C}(10 \mathrm{~A})-\mathrm{C}(9 \mathrm{~A})$ & \\
\hline $\mathrm{C}(12 \mathrm{~A})-\mathrm{C}(11 \mathrm{~A})-\mathrm{C}(10 \mathrm{~A})$ & \\
\hline $\mathrm{C}(12 \mathrm{~A})-\mathrm{C}(11 \mathrm{~A})-\mathrm{H}(11 \mathrm{~B})$ & \\
\hline $\mathrm{C}(10 \mathrm{~A})-\mathrm{C}(11 \mathrm{~A})-\mathrm{H}(11 \mathrm{~B})$ & \\
\hline
\end{tabular}




\begin{tabular}{|l|l|}
\hline $\mathrm{C}(11 \mathrm{~A})-\mathrm{C}(12 \mathrm{~A})-\mathrm{C}(13 \mathrm{~A})$ & 120.0 \\
\hline $\mathrm{C}(11 \mathrm{~A})-\mathrm{C}(12 \mathrm{~A})-\mathrm{H}(12 \mathrm{~B})$ & 120.0 \\
\hline $\mathrm{C}(13 \mathrm{~A})-\mathrm{C}(12 \mathrm{~A})-\mathrm{H}(12 \mathrm{~B})$ & 120.0 \\
\hline $\mathrm{C}(14 \mathrm{~A})-\mathrm{C}(13 \mathrm{~A})-\mathrm{C}(12 \mathrm{~A})$ & 120.0 \\
\hline $\mathrm{C}(14 \mathrm{~A})-\mathrm{C}(13 \mathrm{~A})-\mathrm{H}(13 \mathrm{~B})$ & 120.0 \\
\hline $\mathrm{C}(12 \mathrm{~A})-\mathrm{C}(13 \mathrm{~A})-\mathrm{H}(13 \mathrm{~B})$ & 120.0 \\
\hline $\mathrm{C}(13 \mathrm{~A})-\mathrm{C}(14 \mathrm{~A})-\mathrm{C}(15 \mathrm{~A})$ & 120.0 \\
\hline $\mathrm{C}(13 \mathrm{~A})-\mathrm{C}(14 \mathrm{~A})-\mathrm{H}(14 \mathrm{~B})$ & 120.0 \\
\hline $\mathrm{C}(15 \mathrm{~A})-\mathrm{C}(14 \mathrm{~A})-\mathrm{H}(14 \mathrm{~B})$ & 120.0 \\
\hline $\mathrm{C}(14 \mathrm{~A})-\mathrm{C}(15 \mathrm{~A})-\mathrm{C}(10 \mathrm{~A})$ & 120.0 \\
\hline $\mathrm{C}(14 \mathrm{~A})-\mathrm{C}(15 \mathrm{~A})-\mathrm{C}(16 \mathrm{~A})$ & $110.6(14)$ \\
\hline $\mathrm{C}(10 \mathrm{~A})-\mathrm{C}(15 \mathrm{~A})-\mathrm{C}(16 \mathrm{~A})$ & $125.5(14)$ \\
\hline $\mathrm{C}(15 \mathrm{~A})-\mathrm{C}(16 \mathrm{~A})-\mathrm{H}(16 \mathrm{D})$ & 109.5 \\
\hline $\mathrm{C}(15 \mathrm{~A})-\mathrm{C}(16 \mathrm{~A})-\mathrm{H}(16 \mathrm{E})$ & 109.5 \\
\hline $\mathrm{H}(16 \mathrm{D})-\mathrm{C}(16 \mathrm{~A})-\mathrm{H}(16 \mathrm{E})$ & 109.5 \\
\hline $\mathrm{C}(15 \mathrm{~A})-\mathrm{C}(16 \mathrm{~A})-\mathrm{H}(16 \mathrm{~F})$ & 109.5 \\
\hline $\mathrm{H}(16 \mathrm{D})-\mathrm{C}(16 \mathrm{~A})-\mathrm{H}(16 \mathrm{~F})$ & 109.5 \\
\hline $\mathrm{H}(16 \mathrm{E})-\mathrm{C}(16 \mathrm{~A})-\mathrm{H}(16 \mathrm{~F})$ & 109.5 \\
\hline $\mathrm{O}(5)-\mathrm{B}(1)-\mathrm{O}(1)$ & $110.7(3)$ \\
\hline $\mathrm{O}(5)-\mathrm{B}(1)-\mathrm{O}(3)$ & $113.4(3)$ \\
\hline $\mathrm{O}(1)-\mathrm{B}(1)-\mathrm{O}(3)$ & $107.7(3)$ \\
\hline $\mathrm{O}(1)-\mathrm{B}(1)-\mathrm{O}(5 \mathrm{~A})$ & $112.2(5)$ \\
\hline $\mathrm{O}(3)-\mathrm{B}(1)-\mathrm{O}(5 \mathrm{~A})$ & $89.3(5)$ \\
\hline $\mathrm{O}(5)-\mathrm{B}(1)-\mathrm{C}(1)$ & $104.2(3)$ \\
\hline $\mathrm{O}(1)-\mathrm{B}(1)-\mathrm{C}(1)$ & $109.6(3)$ \\
\hline $\mathrm{O}(3)-\mathrm{B}(1)-\mathrm{C}(1)$ & $111.1(3)$ \\
\hline $\mathrm{O}(1)-\mathrm{B}(1)-\mathrm{C}(1 \mathrm{~A})$ & $109.6(3)$ \\
\hline $\mathrm{O}(3)-\mathrm{B}(1)-\mathrm{C}(1 \mathrm{~A})$ & $111.1(3)$ \\
\hline $\mathrm{O}(5 \mathrm{~A})-\mathrm{B}(1)-\mathrm{C}(1 \mathrm{~A})$ & $124.2(6)$ \\
\hline $\mathrm{C}(15)-\mathrm{C}(16)-\mathrm{H}(16 \mathrm{C})$ & 109.5 \\
\hline $\mathrm{H}(16 \mathrm{~A})-\mathrm{C}(16)-\mathrm{H}(16 \mathrm{C})$ & 109.5 \\
\hline $\mathrm{H}(16 \mathrm{~B})-\mathrm{C}(16)-\mathrm{H}(16 \mathrm{C})$ & 109.5 \\
\hline $\mathrm{C}(11 \mathrm{~A})-\mathrm{C}(10 \mathrm{~A})-\mathrm{C}(15 \mathrm{~A})$ & 120.0 \\
\hline $\mathrm{C}(11 \mathrm{~A})-\mathrm{C}(10 \mathrm{~A})-\mathrm{C}(9 \mathrm{~A})$ & $107.9(16)$ \\
\hline $\mathrm{C}(15 \mathrm{~A})-\mathrm{C}(10 \mathrm{~A})-\mathrm{C}(9 \mathrm{~A})$ & $132.0(16)$ \\
\hline $\mathrm{C}(12 \mathrm{~A})-\mathrm{C}(11 \mathrm{~A})-\mathrm{C}(10 \mathrm{~A})$ & 120.0 \\
\hline $\mathrm{C}(12 \mathrm{~A})-\mathrm{C}(11 \mathrm{~A})-\mathrm{H}(11 \mathrm{~B})$ & 120.0 \\
\hline $\mathrm{C}(10 \mathrm{~A})-\mathrm{C}(11 \mathrm{~A})-\mathrm{H}(11 \mathrm{~B})$ & 120.0 \\
\hline $\mathrm{C}(11 \mathrm{~A})-\mathrm{C}(12 \mathrm{~A})-\mathrm{C}(13 \mathrm{~A})$ & 120.0 \\
\hline $\mathrm{C}(11 \mathrm{~A})-\mathrm{C}(12 \mathrm{~A})-\mathrm{H}(12 \mathrm{~B})$ & 120.0 \\
\hline $\mathrm{C}(13 \mathrm{~A})-\mathrm{C}(12 \mathrm{~A})-\mathrm{H}(12 \mathrm{~B})$ & 120.0 \\
\hline $\mathrm{C}(14 \mathrm{~A})-\mathrm{C}(13 \mathrm{~A})-\mathrm{C}(12 \mathrm{~A})$ & 120.0 \\
\hline $\mathrm{C}(14 \mathrm{~A})-\mathrm{C}(13 \mathrm{~A})-\mathrm{H}(13 \mathrm{~B})$ & \\
\hline $\mathrm{C}(12 \mathrm{~A})-\mathrm{C}(13 \mathrm{~A})-\mathrm{H}(13 \mathrm{~B})$ & \\
\hline $\mathrm{C}(13 \mathrm{~A})-\mathrm{C}(14 \mathrm{~A})-\mathrm{C}(15 \mathrm{~A})$ & \\
\hline $\mathrm{C}(13 \mathrm{~A})-\mathrm{C}(14 \mathrm{~A})-\mathrm{H}(14 \mathrm{~B})$ & \\
\hline
\end{tabular}




\begin{tabular}{|l|l|}
\hline $\mathrm{C}(15 \mathrm{~A})-\mathrm{C}(14 \mathrm{~A})-\mathrm{H}(14 \mathrm{~B})$ & 120.0 \\
\hline $\mathrm{C}(14 \mathrm{~A})-\mathrm{C}(15 \mathrm{~A})-\mathrm{C}(10 \mathrm{~A})$ & 120.0 \\
\hline $\mathrm{C}(14 \mathrm{~A})-\mathrm{C}(15 \mathrm{~A})-\mathrm{C}(16 \mathrm{~A})$ & $110.6(14)$ \\
\hline $\mathrm{C}(10 \mathrm{~A})-\mathrm{C}(15 \mathrm{~A})-\mathrm{C}(16 \mathrm{~A})$ & $125.5(14)$ \\
\hline $\mathrm{C}(15 \mathrm{~A})-\mathrm{C}(16 \mathrm{~A})-\mathrm{H}(16 \mathrm{D})$ & 109.5 \\
\hline $\mathrm{C}(15 \mathrm{~A})-\mathrm{C}(16 \mathrm{~A})-\mathrm{H}(16 \mathrm{E})$ & 109.5 \\
\hline $\mathrm{H}(16 \mathrm{D})-\mathrm{C}(16 \mathrm{~A})-\mathrm{H}(16 \mathrm{E})$ & 109.5 \\
\hline $\mathrm{C}(15 \mathrm{~A})-\mathrm{C}(16 \mathrm{~A})-\mathrm{H}(16 \mathrm{~F})$ & 109.5 \\
\hline $\mathrm{H}(16 \mathrm{D})-\mathrm{C}(16 \mathrm{~A})-\mathrm{H}(16 \mathrm{~F})$ & 109.5 \\
\hline $\mathrm{H}(16 \mathrm{E})-\mathrm{C}(16 \mathrm{~A})-\mathrm{H}(16 \mathrm{~F})$ & 109.5 \\
\hline $\mathrm{O}(5)-\mathrm{B}(1)-\mathrm{O}(1)$ & $110.7(3)$ \\
\hline $\mathrm{O}(5)-\mathrm{B}(1)-\mathrm{O}(3)$ & $113.4(3)$ \\
\hline $\mathrm{O}(1)-\mathrm{B}(1)-\mathrm{O}(3)$ & $107.7(3)$ \\
\hline $\mathrm{O}(1)-\mathrm{B}(1)-\mathrm{O}(5 \mathrm{~A})$ & $112.2(5)$ \\
\hline $\mathrm{O}(3)-\mathrm{B}(1)-\mathrm{O}(5 \mathrm{~A})$ & $89.3(5)$ \\
\hline $\mathrm{O}(5)-\mathrm{B}(1)-\mathrm{C}(1)$ & $104.2(3)$ \\
\hline $\mathrm{O}(1)-\mathrm{B}(1)-\mathrm{C}(1)$ & $109.6(3)$ \\
\hline $\mathrm{O}(3)-\mathrm{B}(1)-\mathrm{C}(1)$ & $111.1(3)$ \\
\hline $\mathrm{O}(1)-\mathrm{B}(1)-\mathrm{C}(1 \mathrm{~A})$ & $109.6(3)$ \\
\hline $\mathrm{O}(3)-\mathrm{B}(1)-\mathrm{C}(1 \mathrm{~A})$ & $111.1(3)$ \\
\hline $\mathrm{O}(5 \mathrm{~A})-\mathrm{B}(1)-\mathrm{C}(1 \mathrm{~A})$ & $124.2(6)$ \\
\hline
\end{tabular}

Table S-6. Anisotropic displacement parameters $(\AA 2 \times 103)$ for $2 c$. The anisotropic displacement factor exponent takes the form: $-2 p^{2}\left[h^{2} a^{* 2} U^{11}+\ldots+2 h k a^{*} b^{*} U^{12}\right]$.

\begin{tabular}{|l|l|l|l|l|l|l|}
\hline & $\mathbf{U}^{\mathbf{1 1}}$ & $\mathbf{U}^{\mathbf{2 2}}$ & $\mathbf{U}^{\mathbf{3 3}}$ & $\mathbf{U}^{\mathbf{2 3}}$ & $\mathbf{U}^{\mathbf{1 3}}$ & $\mathbf{U}^{\mathbf{1 2}}$ \\
\hline $\mathrm{C}(2)$ & $65(2)$ & $45(2)$ & $34(2)$ & $-6(1)$ & $18(1)$ & $4(2)$ \\
\hline $\mathrm{O}(1)$ & $56(2)$ & $22(1)$ & $116(2)$ & $0(1)$ & $48(2)$ & $3(1)$ \\
\hline $\mathrm{O}(2)$ & $55(1)$ & $32(1)$ & $85(2)$ & $9(1)$ & $35(1)$ & $-3(1)$ \\
\hline $\mathrm{O}(3)$ & $54(1)$ & $33(1)$ & $62(1)$ & $-13(1)$ & $30(1)$ & $2(1)$ \\
\hline $\mathrm{O}(4)$ & $70(2)$ & $48(1)$ & $37(1)$ & $-4(1)$ & $20(1)$ & $-2(1)$ \\
\hline $\mathrm{N}(1)$ & $43(1)$ & $26(1)$ & $38(1)$ & $5(1)$ & $16(1)$ & $4(1)$ \\
\hline $\mathrm{C}(3)$ & $49(2)$ & $30(2)$ & $52(2)$ & $13(1)$ & $28(1)$ & $4(1)$ \\
\hline $\mathrm{C}(4)$ & $51(2)$ & $27(2)$ & $71(2)$ & $14(2)$ & $30(2)$ & $6(1)$ \\
\hline $\mathrm{C}(5)$ & $47(2)$ & $20(1)$ & $34(1)$ & $4(1)$ & $17(1)$ & $3(1)$ \\
\hline $\mathrm{C}(6)$ & $47(2)$ & $26(1)$ & $45(2)$ & $-5(1)$ & $22(1)$ & $-5(1)$ \\
\hline $\mathrm{C}(1)$ & $43(2)$ & $48(2)$ & $60(2)$ & $7(2)$ & $14(1)$ & $11(1)$ \\
\hline $\mathrm{O}(5)$ & $39(2)$ & $42(2)$ & $61(3)$ & $-12(2)$ & $11(2)$ & $11(2)$ \\
\hline $\mathrm{O}(6)$ & $46(2)$ & $30(2)$ & $55(2)$ & $-8(1)$ & $20(2)$ & $4(1)$ \\
\hline $\mathrm{C}(7)$ & $55(3)$ & $49(3)$ & $66(3)$ & $5(2)$ & $12(2)$ & $1(2)$ \\
\hline $\mathrm{C}(8)$ & $55(3)$ & $101(5)$ & $96(5)$ & $10(4)$ & $-4(3)$ & $-4(3)$ \\
\hline $\mathrm{C}(9)$ & $47(3)$ & $38(3)$ & $55(3)$ & $10(3)$ & $22(2)$ & $19(2)$ \\
\hline $\mathrm{C}(1 \mathrm{~A})$ & $43(2)$ & $48(2)$ & $60(2)$ & $7(2)$ & $14(1)$ & $11(1)$ \\
\hline $\mathrm{O}(5 \mathrm{~A})$ & $46(4)$ & $42(4)$ & $68(5)$ & $10(5)$ & $24(4)$ & $14(3)$ \\
\hline $\mathrm{O}(6 \mathrm{~A})$ & $81(6)$ & $95(7)$ & $120(8)$ & $42(6)$ & $23(6)$ & $20(6)$ \\
\hline $\mathrm{C}(7 \mathrm{~A})$ & $45(4)$ & $56(5)$ & $35(4)$ & $-3(4)$ & $11(3)$ & $16(4)$ \\
\hline
\end{tabular}




\begin{tabular}{|l|l|l|l|l|l|l|}
\hline $\mathrm{C}(8 \mathrm{~A})$ & $52(6)$ & $93(8)$ & $55(6)$ & $-4(6)$ & $2(5)$ & $14(6)$ \\
\hline $\mathrm{C}(9 \mathrm{~A})$ & $50(5)$ & $41(5)$ & $74(6)$ & $16(6)$ & $18(5)$ & $4(4)$ \\
\hline $\mathrm{C}(10)$ & $47(3)$ & $81(4)$ & $111(4)$ & $16(3)$ & $38(3)$ & $30(3)$ \\
\hline $\mathrm{C}(11)$ & $58(3)$ & $115(5)$ & $142(6)$ & $16(4)$ & $20(4)$ & $21(3)$ \\
\hline $\mathrm{C}(12)$ & $66(3)$ & $142(6)$ & $167(6)$ & $10(5)$ & $12(4)$ & $26(4)$ \\
\hline $\mathrm{C}(13)$ & $75(4)$ & $158(6)$ & $182(6)$ & $-3(5)$ & $35(4)$ & $45(4)$ \\
\hline $\mathrm{C}(14)$ & $92(4)$ & $147(6)$ & $180(6)$ & $-16(5)$ & $38(4)$ & $56(4)$ \\
\hline $\mathrm{C}(15)$ & $82(3)$ & $117(5)$ & $160(5)$ & $-8(4)$ & $40(4)$ & $42(4)$ \\
\hline $\mathrm{C}(16)$ & $131(7)$ & $102(8)$ & $187(11)$ & $-31(8)$ & $31(9)$ & $32(6)$ \\
\hline $\mathrm{C}(10 \mathrm{~A})$ & $54(4)$ & $98(5)$ & $131(6)$ & $7(5)$ & $37(4)$ & $31(4)$ \\
\hline $\mathrm{C}(11 \mathrm{~A})$ & $58(4)$ & $126(6)$ & $145(6)$ & $5(5)$ & $23(5)$ & $22(5)$ \\
\hline $\mathrm{C}(12 \mathrm{~A})$ & $62(5)$ & $142(6)$ & $162(7)$ & $7(6)$ & $23(5)$ & $28(5)$ \\
\hline $\mathrm{C}(13 \mathrm{~A})$ & $66(5)$ & $143(6)$ & $169(7)$ & $3(6)$ & $27(5)$ & $48(5)$ \\
\hline $\mathrm{C}(14 \mathrm{~A})$ & $79(5)$ & $130(6)$ & $168(7)$ & $1(6)$ & $42(5)$ & $52(5)$ \\
\hline $\mathrm{C}(15 \mathrm{~A})$ & $76(4)$ & $112(5)$ & $153(6)$ & $-2(5)$ & $42(5)$ & $43(4)$ \\
\hline $\mathrm{C}(16 \mathrm{~A})$ & $100(9)$ & $116(11)$ & $129(11)$ & $-40(10)$ & $72(9)$ & $28(9)$ \\
\hline $\mathrm{B}(1)$ & $42(2)$ & $27(2)$ & $75(3)$ & $2(2)$ & $26(2)$ & $4(1)$ \\
\hline
\end{tabular}

Table S-7. Hydrogen coordinates ( x 104) and isotropic displacement parameters $\left(\AA^{2} \times 10^{3}\right)$ for 2c.

\begin{tabular}{|l|l|l|l|l|}
\hline & $\mathbf{x}$ & $\mathbf{y}$ & $\mathbf{z}$ & $\mathbf{U}(\mathbf{e q})$ \\
\hline $\mathrm{H}(2 \mathrm{~A})$ & 8396 & -1932 & 2246 & 70 \\
\hline $\mathrm{H}(2 \mathrm{~B})$ & 8625 & -219 & 1525 & 70 \\
\hline $\mathrm{H}(2 \mathrm{C})$ & 9229 & -1486 & 2335 & 70 \\
\hline $\mathrm{H}(3 \mathrm{~A})$ & 9268 & 2469 & 2234 & 49 \\
\hline $\mathrm{H}(3 \mathrm{~B})$ & 9784 & 1651 & 3306 & 49 \\
\hline $\mathrm{H}(5 \mathrm{~A})$ & 9477 & -763 & 4266 & 39 \\
\hline $\mathrm{H}(5 \mathrm{~B})$ & 8687 & -1691 & 4059 & 39 \\
\hline $\mathrm{H}(1)$ & 7927 & 2462 & 2278 & 60 \\
\hline $\mathrm{H}(7 \mathrm{~A})$ & 7431 & -527 & 3422 & 69 \\
\hline $\mathrm{H}(8 \mathrm{~A})$ & 6858 & 1008 & 1398 & 105 \\
\hline $\mathrm{H}(8 \mathrm{~B})$ & 6509 & -770 & 2000 & 105 \\
\hline $\mathrm{H}(1 \mathrm{~A})$ & 7722 & 580 & 3174 & 60 \\
\hline $\mathrm{H}(7 \mathrm{AA})$ & 7863 & 3015 & 1547 & 54 \\
\hline $\mathrm{H}(8 \mathrm{~A} 1)$ & 6730 & 680 & 1769 & 82 \\
\hline $\mathrm{H}(8 \mathrm{~A} 2)$ & 6798 & 2015 & 731 & 82 \\
\hline $\mathrm{H}(11 \mathrm{~A})$ & 6170 & 4011 & 3086 & 127 \\
\hline $\mathrm{H}(12 \mathrm{~A})$ & 4949 & 4812 & 2804 & 153 \\
\hline $\mathrm{H}(13 \mathrm{~A})$ & 4595 & 7569 & 3679 & 165 \\
\hline $\mathrm{H}(14 \mathrm{~A})$ & 5461 & 9525 & 4837 & 167 \\
\hline $\mathrm{H}(16 \mathrm{~A})$ & 7319 & 8929 & 5420 & 212 \\
\hline $\mathrm{H}(16 \mathrm{~B})$ & 6754 & 10713 & 5022 & 212 \\
\hline $\mathrm{H}(16 \mathrm{C})$ & 6711 & 9403 & 6038 & 212 \\
\hline $\mathrm{H}(11 \mathrm{~B})$ & 6137 & 3601 & 3335 & 132 \\
\hline
\end{tabular}




\begin{tabular}{|l|l|l|l|l|}
\hline $\mathrm{H}(12 \mathrm{~B})$ & 4963 & 3766 & 3556 & 148 \\
\hline $\mathrm{H}(13 \mathrm{~B})$ & 4640 & 6354 & 4573 & 152 \\
\hline $\mathrm{H}(14 \mathrm{~B})$ & 5490 & 8776 & 5369 & 148 \\
\hline $\mathrm{H}(16 \mathrm{D})$ & 7377 & 8832 & 5690 & 161 \\
\hline $\mathrm{H}(16 \mathrm{E})$ & 6740 & 10335 & 5774 & 161 \\
\hline $\mathrm{H}(16 \mathrm{~F})$ & 6882 & 8335 & 6485 & 161 \\
\hline $\mathrm{H}(2 \mathrm{~A})$ & 8396 & -1932 & 2246 & 70 \\
\hline $\mathrm{H}(2 \mathrm{~B})$ & 8625 & -219 & 1525 & 70 \\
\hline $\mathrm{H}(2 \mathrm{C})$ & 9229 & -1486 & 2335 & 70 \\
\hline $\mathrm{H}(3 \mathrm{~A})$ & 9268 & 2469 & 2234 & 49 \\
\hline $\mathrm{H}(3 \mathrm{~B})$ & 9784 & 1651 & 3306 & 49 \\
\hline $\mathrm{H}(5 \mathrm{~A})$ & 9477 & -763 & 4266 & 39 \\
\hline $\mathrm{H}(5 \mathrm{~B})$ & 8687 & -1691 & 4059 & 39 \\
\hline $\mathrm{H}(1)$ & 7927 & 2462 & 2278 & 60 \\
\hline $\mathrm{H}(7 \mathrm{~A})$ & 7431 & -527 & 3422 & 69 \\
\hline $\mathrm{H}(8 \mathrm{~A})$ & 6858 & 1008 & 1398 & 105 \\
\hline $\mathrm{H}(8 \mathrm{~B})$ & 6509 & -770 & 2000 & 105 \\
\hline $\mathrm{H}(1 \mathrm{~A})$ & 7722 & 580 & 3174 & 60 \\
\hline $\mathrm{H}(7 \mathrm{AA})$ & 7863 & 3015 & 1547 & 54 \\
\hline $\mathrm{H}(8 \mathrm{~A} 1)$ & 6730 & 680 & 1769 & 82 \\
\hline $\mathrm{H}(8 \mathrm{~A} 2)$ & 6798 & 2015 & 731 & 82 \\
\hline $\mathrm{H}(11 \mathrm{~A})$ & 6170 & 4011 & 3086 & 127 \\
\hline $\mathrm{H}(12 \mathrm{~A})$ & 4949 & 4812 & 2804 & 153 \\
\hline $\mathrm{H}(13 \mathrm{~A})$ & 4595 & 7569 & 3679 & 165 \\
\hline $\mathrm{H}(14 \mathrm{~A})$ & 5461 & 9525 & 4837 & 167 \\
\hline $\mathrm{H}(16 \mathrm{~A})$ & 7319 & 8929 & 5420 & 212 \\
\hline $\mathrm{H}(16 \mathrm{~B})$ & 6754 & 10713 & 5022 & 212 \\
\hline $\mathrm{H}(16 \mathrm{C})$ & 6711 & 9403 & 6038 & 212 \\
\hline $\mathrm{H}(11 \mathrm{~B})$ & 6137 & 3601 & 3335 & 132 \\
\hline $\mathrm{H}(12 \mathrm{~B})$ & 4963 & 3766 & 3556 & 148 \\
\hline $\mathrm{H}(13 \mathrm{~B})$ & 4640 & 6354 & 4573 & 152 \\
\hline $\mathrm{H}(14 \mathrm{~B})$ & 5490 & 8776 & 5369 & 148 \\
\hline $\mathrm{H}(16 \mathrm{D})$ & 7377 & 8832 & 5690 & 161 \\
\hline $\mathrm{H}(16 \mathrm{E})$ & 6740 & 10335 & 5774 & 161 \\
\hline $\mathrm{H}(16 \mathrm{~F})$ & 6882 & 8335 & 6485 & 161 \\
\hline $\mathrm{H}(8 \mathrm{~B})$ & 6509 & -770 & 2000 & 105 \\
\hline $\mathrm{H}(1 \mathrm{~A})$ & 7722 & 580 & 3174 & 60 \\
\hline $\mathrm{H}(7 \mathrm{AA})$ & 7863 & 3015 & 1547 & 54 \\
\hline $\mathrm{H}(8 \mathrm{~A} 1)$ & 6730 & 680 & 1769 & 82 \\
\hline $\mathrm{H}(8 \mathrm{~A} 2)$ & 6798 & 2015 & 731 & 82 \\
\hline $\mathrm{H}(11 \mathrm{~A})$ & 6170 & 4011 & 3086 & 127 \\
\hline $\mathrm{H}(12 \mathrm{~A})$ & 4949 & 4812 & 2804 & 153 \\
\hline $\mathrm{H}(13 \mathrm{~A})$ & 4595 & 7569 & 3679 & 165 \\
\hline $\mathrm{H}(14 \mathrm{~A})$ & 5461 & 9525 & 4837 & 167 \\
\hline $\mathrm{H}(16 \mathrm{~A})$ & 7319 & 8929 & 5420 & 212 \\
\hline $\mathrm{H}(16 \mathrm{~B})$ & 6754 & 10713 & 5022 & 212 \\
\hline & & & & \\
\hline
\end{tabular}




\begin{tabular}{|l|l|l|l|l|}
\hline $\mathrm{H}(16 \mathrm{C})$ & 6711 & 9403 & 6038 & 212 \\
\hline $\mathrm{H}(11 \mathrm{~B})$ & 6137 & 3601 & 3335 & 132 \\
\hline $\mathrm{H}(12 \mathrm{~B})$ & 4963 & 3766 & 3556 & 148 \\
\hline $\mathrm{H}(13 \mathrm{~B})$ & 4640 & 6354 & 4573 & 152 \\
\hline $\mathrm{H}(14 \mathrm{~B})$ & 5490 & 8776 & 5369 & 148 \\
\hline $\mathrm{H}(16 \mathrm{D})$ & 7377 & 8832 & 5690 & 161 \\
\hline $\mathrm{H}(16 \mathrm{E})$ & 6740 & 10335 & 5774 & 161 \\
\hline $\mathrm{H}(16 \mathrm{~F})$ & 6882 & 8335 & 6485 & 161 \\
\hline
\end{tabular}

Table S-8. Torsion angles $\left[^{\circ}\right]$ for $\mathbf{2 c}$.

\begin{tabular}{|c|c|}
\hline $\mathrm{C}(5)-\mathrm{N}(1)-\mathrm{C}(3)-\mathrm{C}(4)$ & $-81.7(3)$ \\
\hline $\mathrm{C}(2)-\mathrm{N}(1)-\mathrm{C}(3)-\mathrm{C}(4)$ & $161.5(3)$ \\
\hline $\mathrm{C}(1 \mathrm{~A})-\mathrm{N}(1)-\mathrm{C}(3)-\mathrm{C}(4)$ & $39.5(4)$ \\
\hline $\mathrm{C}(1)-\mathrm{N}(1)-\mathrm{C}(3)-\mathrm{C}(4)$ & $39.5(4)$ \\
\hline $\mathrm{B}(1)-\mathrm{O}(1)-\mathrm{C}(4)-\mathrm{O}(2)$ & $-166.2(3)$ \\
\hline $\mathrm{B}(1)-\mathrm{O}(1)-\mathrm{C}(4)-\mathrm{C}(3)$ & $20.0(5)$ \\
\hline $\mathrm{N}(1)-\mathrm{C}(3)-\mathrm{C}(4)-\mathrm{O}(2)$ & $168.6(3)$ \\
\hline $\mathrm{N}(1)-\mathrm{C}(3)-\mathrm{C}(4)-\mathrm{O}(1)$ & $-17.4(5)$ \\
\hline $\mathrm{C}(3)-\mathrm{N}(1)-\mathrm{C}(5)-\mathrm{C}(6)$ & $76.5(3)$ \\
\hline $\mathrm{C}(2)-\mathrm{N}(1)-\mathrm{C}(5)-\mathrm{C}(6)$ & $-167.0(3)$ \\
\hline$C(1 \mathrm{~A})-\mathrm{N}(1)-\mathrm{C}(5)-\mathrm{C}(6)$ & $-44.8(3)$ \\
\hline $\mathrm{C}(1)-\mathrm{N}(1)-\mathrm{C}(5)-\mathrm{C}(6)$ & $-44.8(3)$ \\
\hline $\mathrm{B}(1)-\mathrm{O}(3)-\mathrm{C}(6)-\mathrm{O}(4)$ & $170.5(3)$ \\
\hline $\mathrm{B}(1)-\mathrm{O}(3)-\mathrm{C}(6)-\mathrm{C}(5)$ & $-13.5(4)$ \\
\hline $\mathrm{N}(1)-\mathrm{C}(5)-\mathrm{C}(6)-\mathrm{O}(4)$ & $-165.5(3)$ \\
\hline $\mathrm{N}(1)-\mathrm{C}(5)-\mathrm{C}(6)-\mathrm{O}(3)$ & $18.3(4)$ \\
\hline $\mathrm{C}(3)-\mathrm{N}(1)-\mathrm{C}(1)-\mathrm{C}(7)$ & $170.3(4)$ \\
\hline $\mathrm{C}(5)-\mathrm{N}(1)-\mathrm{C}(1)-\mathrm{C}(7)$ & $-67.8(4)$ \\
\hline $\mathrm{C}(2)-\mathrm{N}(1)-\mathrm{C}(1)-\mathrm{C}(7)$ & $51.5(4)$ \\
\hline $\mathrm{C}(3)-\mathrm{N}(1)-\mathrm{C}(1)-\mathrm{B}(1)$ & $-59.7(3)$ \\
\hline $\mathrm{C}(5)-\mathrm{N}(1)-\mathrm{C}(1)-\mathrm{B}(1)$ & $62.2(3)$ \\
\hline $\mathrm{C}(2)-\mathrm{N}(1)-\mathrm{C}(1)-\mathrm{B}(1)$ & $-178.5(2)$ \\
\hline $\mathrm{N}(1)-\mathrm{C}(1)-\mathrm{C}(7)-\mathrm{C}(8)$ & $-116.6(7)$ \\
\hline $\mathrm{B}(1)-\mathrm{C}(1)-\mathrm{C}(7)-\mathrm{C}(8)$ & $119.2(7)$ \\
\hline $\mathrm{B}(1)-\mathrm{O}(5)-\mathrm{C}(9)-\mathrm{O}(6)$ & $12.7(11)$ \\
\hline $\mathrm{B}(1)-\mathrm{O}(5)-\mathrm{C}(9)-\mathrm{C}(10)$ & $-174.3(6)$ \\
\hline $\mathrm{C}(3)-\mathrm{N}(1)-\mathrm{C}(1 \mathrm{~A})-\mathrm{C}(7 \mathrm{~A})$ & $79.1(7)$ \\
\hline $\mathrm{C}(5)-\mathrm{N}(1)-\mathrm{C}(1 \mathrm{~A})-\mathrm{C}(7 \mathrm{~A})$ & $-159.0(6)$ \\
\hline $\mathrm{C}(2)-\mathrm{N}(1)-\mathrm{C}(1 \mathrm{~A})-\mathrm{C}(7 \mathrm{~A})$ & $-39.8(7)$ \\
\hline $\mathrm{C}(3)-\mathrm{N}(1)-\mathrm{C}(1 \mathrm{~A})-\mathrm{B}(1)$ & $-59.7(3)$ \\
\hline $\mathrm{C}(5)-\mathrm{N}(1)-\mathrm{C}(1 \mathrm{~A})-\mathrm{B}(1)$ & $62.2(3)$ \\
\hline $\mathrm{C}(2)-\mathrm{N}(1)-\mathrm{C}(1 \mathrm{~A})-\mathrm{B}(1)$ & $-178.5(2)$ \\
\hline $\mathrm{N}(1)-\mathrm{C}(1 \mathrm{~A})-\mathrm{C}(7 \mathrm{~A})-\mathrm{C}(8 \mathrm{~A})$ & $121.8(12)$ \\
\hline $\mathrm{B}(1)-\mathrm{C}(1 \mathrm{~A})-\mathrm{C}(7 \mathrm{~A})-\mathrm{C}(8 \mathrm{~A})$ & $-106.5(13)$ \\
\hline $\mathrm{B}(1)-\mathrm{O}(5 \mathrm{~A})-\mathrm{C}(9 \mathrm{~A})-\mathrm{O}(6 \mathrm{~A})$ & $-4(3)$ \\
\hline
\end{tabular}




\begin{tabular}{|l|l|}
\hline $\mathrm{B}(1)-\mathrm{O}(5 \mathrm{~A})-\mathrm{C}(9 \mathrm{~A})-\mathrm{C}(10 \mathrm{~A})$ & $166.9(14)$ \\
\hline $\mathrm{O}(6)-\mathrm{C}(9)-\mathrm{C}(10)-\mathrm{C}(11)$ & $175.4(6)$ \\
\hline $\mathrm{O}(5)-\mathrm{C}(9)-\mathrm{C}(10)-\mathrm{C}(11)$ & $2.5(9)$ \\
\hline $\mathrm{O}(6)-\mathrm{C}(9)-\mathrm{C}(10)-\mathrm{C}(15)$ & $-6.1(10)$ \\
\hline $\mathrm{O}(5)-\mathrm{C}(9)-\mathrm{C}(10)-\mathrm{C}(15)$ & $-179.0(5)$ \\
\hline $\mathrm{C}(15)-\mathrm{C}(10)-\mathrm{C}(11)-\mathrm{C}(12)$ & 0.0 \\
\hline $\mathrm{C}(9)-\mathrm{C}(10)-\mathrm{C}(11)-\mathrm{C}(12)$ & $178.5(9)$ \\
\hline $\mathrm{C}(10)-\mathrm{C}(11)-\mathrm{C}(12)-\mathrm{C}(13)$ & 0.0 \\
\hline $\mathrm{C}(11)-\mathrm{C}(12)-\mathrm{C}(13)-\mathrm{C}(14)$ & 0.0 \\
\hline $\mathrm{C}(12)-\mathrm{C}(13)-\mathrm{C}(14)-\mathrm{C}(15)$ & 0.0 \\
\hline $\mathrm{C}(13)-\mathrm{C}(14)-\mathrm{C}(15)-\mathrm{C}(10)$ & 0.0 \\
\hline $\mathrm{C}(13)-\mathrm{C}(14)-\mathrm{C}(15)-\mathrm{C}(16)$ & $176.1(10)$ \\
\hline $\mathrm{C}(11)-\mathrm{C}(10)-\mathrm{C}(15)-\mathrm{C}(14)$ & 0.0 \\
\hline $\mathrm{C}(9)-\mathrm{C}(10)-\mathrm{C}(15)-\mathrm{C}(14)$ & $-178.5(8)$ \\
\hline $\mathrm{C}(11)-\mathrm{C}(10)-\mathrm{C}(15)-\mathrm{C}(16)$ & $-175.7(12)$ \\
\hline $\mathrm{C}(9)-\mathrm{C}(10)-\mathrm{C}(15)-\mathrm{C}(16)$ & $5.8(11)$ \\
\hline $\mathrm{O}(5 \mathrm{~A})-\mathrm{C}(9 \mathrm{~A})-\mathrm{C}(10 \mathrm{~A})-\mathrm{C}(11 \mathrm{~A})$ & $-72(2)$ \\
\hline $\mathrm{O}(6 \mathrm{~A})-\mathrm{C}(9 \mathrm{~A})-\mathrm{C}(10 \mathrm{~A})-\mathrm{C}(11 \mathrm{~A})$ & $98(2)$ \\
\hline $\mathrm{O}(5 \mathrm{~A})-\mathrm{C}(9 \mathrm{~A})-\mathrm{C}(10 \mathrm{~A})-\mathrm{C}(15 \mathrm{~A})$ & $112(2)$ \\
\hline $\mathrm{O}(6 \mathrm{~A})-\mathrm{C}(9 \mathrm{~A})-\mathrm{C}(10 \mathrm{~A})-\mathrm{C}(15 \mathrm{~A})$ & $-78(3)$ \\
\hline $\mathrm{C}(15 \mathrm{~A})-\mathrm{C}(10 \mathrm{~A})-\mathrm{C}(11 \mathrm{~A})-\mathrm{C}(12 \mathrm{~A})$ & 0.0 \\
\hline $\mathrm{C}(9 \mathrm{~A})-\mathrm{C}(10 \mathrm{~A})-\mathrm{C}(11 \mathrm{~A})-\mathrm{C}(12 \mathrm{~A})$ & $-176(2)$ \\
\hline $\mathrm{C}(10 \mathrm{~A})-\mathrm{C}(11 \mathrm{~A})-\mathrm{C}(12 \mathrm{~A})-\mathrm{C}(13 \mathrm{~A})$ & 0.0 \\
\hline $\mathrm{C}(11 \mathrm{~A})-\mathrm{C}(12 \mathrm{~A})-\mathrm{C}(13 \mathrm{~A})-\mathrm{C}(14 \mathrm{~A})$ & 0.0 \\
\hline $\mathrm{C}(12 \mathrm{~A})-\mathrm{C}(13 \mathrm{~A})-\mathrm{C}(14 \mathrm{~A})-\mathrm{C}(15 \mathrm{~A})$ & 0.0 \\
\hline $\mathrm{C}(13 \mathrm{~A})-\mathrm{C}(14 \mathrm{~A})-\mathrm{C}(15 \mathrm{~A})-\mathrm{C}(10 \mathrm{~A})$ & 0.0 \\
\hline $\mathrm{C}(13 \mathrm{~A})-\mathrm{C}(14 \mathrm{~A})-\mathrm{C}(15 \mathrm{~A})-\mathrm{C}(16 \mathrm{~A})$ & $-158.9(19)$ \\
\hline $\mathrm{C}(11 \mathrm{~A})-\mathrm{C}(10 \mathrm{~A})-\mathrm{C}(15 \mathrm{~A})-\mathrm{C}(14 \mathrm{~A})$ & 0.0 \\
\hline $\mathrm{C}(9 \mathrm{~A})-\mathrm{C}(10 \mathrm{~A})-\mathrm{C}(15 \mathrm{~A})-\mathrm{C}(14 \mathrm{~A})$ & $175(3)$ \\
\hline $\mathrm{C}(11 \mathrm{~A})-\mathrm{C}(10 \mathrm{~A})-\mathrm{C}(15 \mathrm{~A})-\mathrm{C}(16 \mathrm{~A})$ & $156(2)$ \\
\hline $\mathrm{C}(9 \mathrm{~A})-\mathrm{C}(10 \mathrm{~A})-\mathrm{C}(15 \mathrm{~A})-\mathrm{C}(16 \mathrm{~A})$ & $-29(2)$ \\
\hline $\mathrm{C}(9)-\mathrm{O}(5)-\mathrm{B}(1)-\mathrm{O}(1)$ & $-54.5(7)$ \\
\hline $\mathrm{C}(9)-\mathrm{O}(5)-\mathrm{B}(1)-\mathrm{O}(3)$ & $66.7(7)$ \\
\hline $\mathrm{C}(9)-\mathrm{O}(5)-\mathrm{B}(1)-\mathrm{C}(1)$ & $-172.3(5)$ \\
\hline $\mathrm{C}(4)-\mathrm{O}(1)-\mathrm{B}(1)-\mathrm{O}(5)$ & $-157.7(4)$ \\
\hline $\mathrm{C}(4)-\mathrm{O}(1)-\mathrm{B}(1)-\mathrm{O}(3)$ & $77.8(4)$ \\
\hline $\mathrm{C}(4)-\mathrm{O}(1)-\mathrm{B}(1)-\mathrm{O}(5 \mathrm{~A})$ & $174.5(6)$ \\
\hline $\mathrm{C}(4)-\mathrm{O}(1)-\mathrm{B}(1)-\mathrm{C}(1)$ & $-43.3(5)$ \\
\hline $\mathrm{C}(4)-\mathrm{O}(1)-\mathrm{B}(1)-\mathrm{C}(1 \mathrm{~A})$ & $-43.3(5)$ \\
\hline $\mathrm{C}(6)-\mathrm{O}(3)-\mathrm{B}(1)-\mathrm{O}(5)$ & $151.3(3)$ \\
\hline $\mathrm{C}(6)-\mathrm{O}(3)-\mathrm{B}(1)-\mathrm{O}(1)$ & $-85.9(3)$ \\
\hline $\mathrm{C}(6)-\mathrm{O}(3)-\mathrm{B}(1)-\mathrm{O}(5 \mathrm{~A})$ & $160.9(5)$ \\
\hline $\mathrm{C}(6)-\mathrm{O}(3)-\mathrm{B}(1)-\mathrm{C}(1)$ & \\
\hline $\mathrm{C}(6)-\mathrm{O}(3)-\mathrm{B}(1)-\mathrm{C}(1 \mathrm{~A})$ & \\
\hline $\mathrm{C}(9 \mathrm{~A})-\mathrm{O}(5 \mathrm{~A})-\mathrm{B}(1)-\mathrm{O}(1)$ & \\
\hline $\mathrm{C}(9 \mathrm{~A})-\mathrm{O}(5 \mathrm{~A})-\mathrm{B}(1)-\mathrm{O}(3)$ & \\
\hline
\end{tabular}




\begin{tabular}{|l|l|}
\hline $\mathrm{C}(9 \mathrm{~A})-\mathrm{O}(5 \mathrm{~A})-\mathrm{B}(1)-\mathrm{C}(1 \mathrm{~A})$ & $-85.6(18)$ \\
\hline $\mathrm{C}(7)-\mathrm{C}(1)-\mathrm{B}(1)-\mathrm{O}(5)$ & $-50.2(5)$ \\
\hline $\mathrm{N}(1)-\mathrm{C}(1)-\mathrm{B}(1)-\mathrm{O}(5)$ & $-179.7(3)$ \\
\hline $\mathrm{C}(7)-\mathrm{C}(1)-\mathrm{B}(1)-\mathrm{O}(1)$ & $-168.7(3)$ \\
\hline $\mathrm{N}(1)-\mathrm{C}(1)-\mathrm{B}(1)-\mathrm{O}(1)$ & $61.8(3)$ \\
\hline $\mathrm{C}(7)-\mathrm{C}(1)-\mathrm{B}(1)-\mathrm{O}(3)$ & $72.3(4)$ \\
\hline $\mathrm{N}(1)-\mathrm{C}(1)-\mathrm{B}(1)-\mathrm{O}(3)$ & $-57.2(3)$ \\
\hline $\mathrm{C}(7 \mathrm{~A})-\mathrm{C}(1 \mathrm{~A})-\mathrm{B}(1)-\mathrm{O}(1)$ & $-75.1(7)$ \\
\hline $\mathrm{N}(1)-\mathrm{C}(1 \mathrm{~A})-\mathrm{B}(1)-\mathrm{O}(1)$ & $61.8(3)$ \\
\hline $\mathrm{C}(7 \mathrm{~A})-\mathrm{C}(1 \mathrm{~A})-\mathrm{B}(1)-\mathrm{O}(3)$ & $166.0(6)$ \\
\hline $\mathrm{N}(1)-\mathrm{C}(1 \mathrm{~A})-\mathrm{B}(1)-\mathrm{O}(3)$ & $-57.2(3)$ \\
\hline $\mathrm{C}(7 \mathrm{~A})-\mathrm{C}(1 \mathrm{~A})-\mathrm{B}(1)-\mathrm{O}(5 \mathrm{~A})$ & $61.7(9)$ \\
\hline $\mathrm{N}(1)-\mathrm{C}(1 \mathrm{~A})-\mathrm{B}(1)-\mathrm{O}(5 \mathrm{~A})$ & $-161.5(6)$ \\
\hline
\end{tabular}

\section{$\underline{\text { References }}$}

1. Chen, M. S.; White, M. C. J. Am. Chem. Soc. 2004, 126, 1346-1347.

2. St. Denis, J. D.; Zajdlik, A.; Tan, J.; Trinchera, P.; Lee, C. F.; He, Z.; Adachi, S.; Yudin, A. K. J. Am. Chem. Soc. 2014, 136, 17669-17673.

3. Willand-Charnley, R.; Fisher, T. J.; Johnson, B. M.; Dussault, P. H. Org. Lett. 2012, 14, 2242-2245.

4. Keenan, R. M.; Kruse, L. I. A Convenient Synthesis of S-Aryltropones. Synth. Commun. 1989, 19, 793-798.

5. Corless, V. B.; Holownia, A.; Foy, H.; Mendoza-Sanchez, R.; Adachi, S.; Dudding, T.; Yudin, A. K. Org. Lett. 2018, 20, 5300-5303.

6. D’Annibale, A.; D’Auria, M.; Prati, F.; Romagnoli, C.; Stoia, S.; Racioppi, R.; Viggiani, L. Tetrahedron 2013, 69, 3782-3795.

7. Park, K.; Lee, S. Org. Lett. 2015, 17, 1300-1303.

8. Xie, Y.; Hu, J.; Wang, Y.; Xia, C.; Huang, H. J. Am. Chem. Soc. 2012, 134, 20613-20616.

9. Ohshima, T.; Miyamoto, Y.; Ipposhi, J.; Nakahara, Y.; Utsunomiya, M.; Mashima, K. J. Am. Chem. Soc. 2009, 131, 14317-14328.

10. Frisch, M. J.; Trucks, G. W.; Schlegel, H. B.; Scuseria, G. E.; Robb, M. A.; Cheeseman, J. R.; Scalmani, G.; Barone, V.; Mennucci, B.; Petersson, G. A.; Nakatsuji, H.; Caricato, M.; Li, X.; Hratchian, H. P.; Izmaylov, A. F.; Bloino, J.; Zheng, G.; Sonnenberg, J. L.; Hada, M.; Ehara, M.; Toyota, K.; Fukuda, R.; Hasegawa, J.; Ishida, M.; Nakajima, T.; Honda, Y.; Kitao, O.; Nakai, H.; Vreven, T.; Montgomery, J. A., Jr.; Peralta, J. E.; Ogliaro, F.; Bearpark, M.; Heyd, J. J.; Brothers, E.; Kudin, K. N.; Staroverov, V. N.; Kobayashi, R.; 
Normand, J.; Raghavachari, K.; Rendell, A.; Burant, J. C.; Iyengar, S. S.; Tomasi, J.; Cossi, M.; Rega, N.; Millam, J. M.; Klene, M.; Knox, J. E.; Cross, J. B.; Bakken, V.; Adamo, C.; Jaramillo, J.; Gomperts, R.; Stratmann, R. E.; Yazyev, O.; Austin, A. J.; Cammi, R.; Pomelli, C.; Ochterski, J. W.; Martin, R. L.; Morokuma, K.; Zakrzewski, V. G.; Voth, G. A.; Salvador, P.; Dannenberg, J. J.; Dapprich, S.; Daniels, A. D.; Farkas, O.; Foresman, J. B.; Ortiz, J. V.; Cioslowski, J.; Fox, D. J. Gaussian 09, revision D.01; Gaussian, Inc.: Wallingford, CT, 2013.

11. Lee, C.; Yang, W.; Parr, R. G. Phys. Rev. B: Condens. Matter Mater. Phys. 1988, 37, 785789. (b) Becke, A. D. J. Chem. Phys. 1993, 98, 5648-5652. (c) Stephens, P. J.; Devlin, F. J.; Chabalowski, C. F.; Frisch, M. J. J. Phys. Chem. 1994, 98, 11623-11627.

12. Cancès, E.; Mennucci, B.; Tomasi, J. J. Chem. Phys. 1997, 107, 3032-3041.

13. Legault, C. Y. CYLview, version 1.0b; Université de Sherbrooke: Quebec, Canada, 2009; http://www.cylview.org. 